\title{
Contribuições à análise de outliers em modelos de equações estruturais
}

\author{
Rodrigo de Souza Bulhões \\ DISSERTAÇÃO APRESENTADA \\ $\mathrm{AO}$ \\ Instituto De Matemática e Estatística \\ DA \\ Universidade DE SÃo Paulo \\ PARA \\ OBTENÇÃO DO TÍTULO \\ DE \\ Mestre em CiÊnCIAS \\ Programa: Estatística \\ Orientadora: Profa. Dra. Lúcia Pereira Barroso
}

Durante o desenvolvimento deste trabalho o autor recebeu auxílio financeiro do CNPq

São Paulo, Maio de 2013 


\section{Contribuições à análise de outliers em modelos de equações estruturais}

Esta versão da dissertação contém as correções e alterações sugeridas pela Comissão Julgadora durante a defesa da versão original do trabalho, realizada em 10/05/2013. Uma cópia da versão original está disponível no

Instituto de Matemática e Estatística da Universidade de São Paulo.

Comissão Julgadora:

- Profa. Dra. Lúcia Pereira Barroso (orientadora) - IME-USP

- Profa. Dra. Denise Aparecida Botter - IME-USP

- Prof. Dr. Manoel Raimundo de Sena Junior - CCEN-UFPE 


\section{Agradecimentos}

Gostaria de expressar o meu vivo e sincero agradecimento a todos que, direta ou indiretamente, contribuíram para a realização deste trabalho, em especial:

À minha orientadora Lúcia, pela cordialidade, paciência e compreensão, por ter aberto a minha rede de contatos no meio acadêmico e, sobretudo, por ter me guiado em todas as etapas da escrita desta dissertação, sempre da melhor maneira possível.

A meus pais Cijara e Geraldo, pelo grande amor e apoio, pelos bons exemplos de honestidade, perseverança e humildade, por sempre acreditarem em mim e me incentivarem a seguir em frente.

A meu irmão Geraldo Filho, por ter me ajudado bastante na fase final do mestrado, me hospedando em seu apartamento em São José dos Campos.

A meu sobrinho Matheus, que me enche de carinho e orgulho.

À Profa. Dra. Irini Moustaki (London School of Economics and Political Science, UK), pelas valiosas sugestões sobre o modo de conduzir os estudos de simulação nesta pesquisa.

A meus colegas da pós-graduação Aníbal e Tiago, pela amizade e colaboração.

A meu querido amigo Denis, que me tratou como um irmão nesses anos que vivi em São Paulo.

A meus "velhos" amigos da Bahia, pela diversão garantida sempre que viajava de férias para casa.

A Deus, por mais esta grande vitória. 


\section{Resumo}

\section{Contribuições à análise de outliers em modelos de equações estruturais}

O Modelo de Equações Estruturais (MEE) é habitualmente ajustado para realizar uma análise confirmatória sobre as conjecturas de um pesquisador acerca do relacionamento entre as variáveis observadas e latentes de algum estudo. Na prática, a maneira mais recorrente de avaliar a qualidade das estimativas de um MEE é a partir de medidas que buscam mensurar o quanto a usual matriz de covariâncias clássicas ou ordinárias se distancia da matriz de covariâncias do modelo ajustado, ou a magnitude do afastamento entre as funções de discrepância do modelo hipotético e do modelo saturado. Entretanto, elas podem não captar problemas no ajuste quando há muitos parâmetros a estimar ou bastantes observações. A fim de detectar irregularidades no ajustamento resultantes do impacto provocado pela presença de outliers no conjunto de dados, este trabalho contemplou alguns indicadores conhecidos na literatura, como também considerou alterações no Índice da Qualidade do Ajuste (ou GFI, de Goodness-of-Fit Index) e no Índice Corrigido da Qualidade do Ajuste (ou AGFI, de Adjusted Goodness-of-Fit Index), ambos nas expressões para estimação de parâmetros pelo método de Máxima Verossimilhança, que consistiram em substituir a tradicional matriz de covariâncias pelas matrizes de covariâncias robustas computadas com os seguintes estimadores: Elipsoide de Volume Mínimo, Covariância de Determinante Mínimo, S, MM e Gnanadesikan-Kettenring Ortogonalizado (GKO). Através de estudos de simulação sobre perturbações de desvios de simetria e excesso de curtose, em baixa e alta frações de contaminação, em diferentes tamanhos de amostra e quantidades de variáveis observadas afetadas, foi possível constatar que as propostas de modificação do GFI e do AGFI adaptadas pelo estimador GKO foram as únicas que conseguiram ser informativas em todas essas situações, devendo-se escolher a primeira ou a segunda respectivamente quando a quantidade de parâmetros a serem estimados é baixa ou elevada.

Palavras-chave: Modelo de Equações Estruturais, outliers, Índice da Qualidade do Ajuste, GFI, Índice Corrigido da Qualidade do Ajuste, AGFI, matrizes de covariâncias robustas, estimador de Gnanadesikan-Kettenring Ortogonalizado. 


\section{Abstract}

\section{Contributions to the analysis of outliers in structural equation models}

The Structural Equation Model (SEM) is usually set to perform a confirmatory analysis on the assumptions of a researcher about the relationship between the observed variables and the latent variables of such a study. In practice, the most iterant way of evaluating the quality of the estimates of a SEM comes either from procedures of measuring how distant the usual classic or ordinary covariance matrix is from the covariance matrix of the adjusted model, or from the magnitude of the hiatus in discrepancy functions of both the hypothetical model and the saturated model. Nevertheless, they may fail to capture problems in the adjustment in the occurrence of either several parameters to estimate or several observations. This study included indicators known in the literature in order to detect irregularities in the adjustment resulting from the impact caused by the presence of outliers in the data set. This study has also considered changes in both the Goodness-ofFit Index (GFI) and the Adjusted Goodness-of-Fit Index (AGFI) in the expressions for parameter estimation by Maximum Likelihood method, which consisted in replacing the traditional covariance matrix by the robust covariance matrices computed through the following estimators: Minimum Volume Ellipsoid, Minimum Covariance Determinant, S, MM and Orthogonalized GnanadesikanKettenring (OGK). Through simulation studies on disturbances of both symmetry deviations and excess kurtosis in both low and high fractions of contamination in different sample sizes and quantities of affected observed variables it has become clear that the proposals of modification of both the GFI and the AGFI adapted by the OGK estimator were the only ones able to be informative in all these situations. It must be considered that GFI or AGFI must be used when the number of parameters to be estimated is either low or high, respectively.

Keywords: Structural Equation Model, outliers, Goodness-of-Fit Index, GFI, Adjusted Goodnessof-Fit Index, AGFI, robust covariance matrices, Orthogonalized Gnanadesikan-Kettenring estimator. 


\section{Sumário}

$\begin{array}{ll}\text { Lista de Figuras } & \text { ix }\end{array}$

Lista de Tabelas $\quad$ xi

1 Introdução $\quad 1$

2 Modelos de Equações Estruturais $\quad 3$

2.1 Formulação matemática . . . . . . . . . . . . . . . . . 3

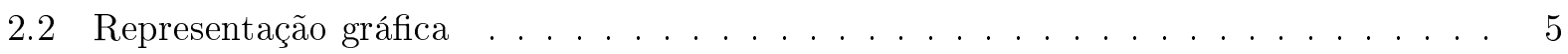

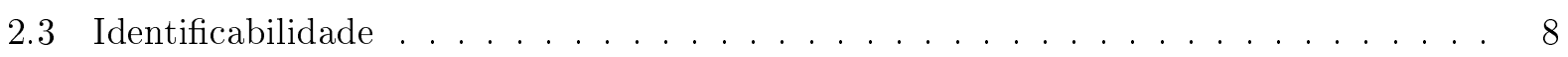

2.4 Métodos de estimação . . . . . . . . . . . . . . . . . . 8

2.4.1 Mínimos Quadrados Ordinários . . . . . . . . . . . . . . . . 9

2.4.2 Mínimos Quadrados Generalizados . . . . . . . . . . . . . . . . . 9

2.4 .3 Máxima Verossimilhança . . . . . . . . . . . . . . . . . . . 10

2.5 Aspectos computacionais . . . . . . . . . . . . . . . . . . . 11

2.6 Pressupostos da modelagem . . . . . . . . . . . . . . . . . . . . 11

3 Análise de Outliers $\quad 13$

3.1 Resíduos . . . . . . . . . . . . . . . . . . . . . . . 13

3.2 Conceitos básicos de robustez . . . . . . . . . . . . . . . . . . . 14

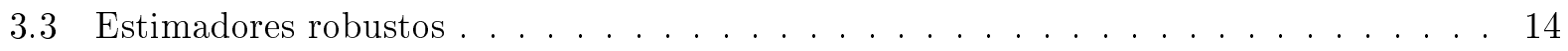

3.3.1 Abordagem exploratória . . . . . . . . . . . . . . . 15

3.3 .2 Abordagem confirmatória . . . . . . . . . . . . . . . 20

4 Indicadores Clássicos e Robustos $\quad 23$

4.1 Indicadores clássicos . . . . . . . . . . . . . . . . . . . 23

4.1.1 Raiz Quadrada Média Padronizada . . . . . . . . . . . . . . . . . . . . 24

4.1.2 Raiz do Erro Quadrático Médio de Aproximação . . . . . . . . . . . . . . . 24

4.1 .3 Índice de Ajuste Normalizado . . . . . . . . . . . . . . . . . 25

4.1 .4 Índice de Ajuste Corrigido . . . . . . . . . . . . . . . . . 25

4.1.5 Índice de Tucker-Lewis ou Índice de Ajuste Não Normalizado . . . . . . . . . 25

4.1 .6 Índice de Ajuste Comparativo . . . . . . . . . . . . . . . . . . . 26

4.1.7 Índice Relativo de Não Centralidade . . . . . . . . . . . . . . . . . . . . 26

4.1 .8 Índices da Qualidade do Ajuste . . . . . . . . . . . . . . . . . . 26

4.1 .9 Índices Corrigidos da Qualidade do Ajuste . . . . . . . . . . . . . . . 27

4.2 Indicadores robustos - propostas alternativas . . . . . . . . . . 27 
5 Simulações $\quad 31$

5.1 Tamanhos de amostra . . . . . . . . . . . . . . . . . 31

5.2 Contaminações por outliers . . . . . . . . . . . . . . . . . . . 32

5.3 Estudos de simulação . . . . . . . . . . . . . . . . . . . 32

5.3.1 Cenário 1: Análise Fatorial Confirmatória - muitos parâmetros a estimar . 34

5.3.2 Cenário 2: Análise Fatorial Confirmatória — poucos parâmetros a estimar . 49

6 Considerações e Trabalhos Futuros $\quad 63$

$\begin{array}{ll}\text { A Derivadas de Matrizes } & 65\end{array}$

$\begin{array}{ll}\text { B Algoritmos de Estimadores Robustos } & 67\end{array}$

B.1 M-Estimador . . . . . . . . . . . . . . . . . 67

B.2 Menor Mediana dos Quadrados e Mediana dos Quadrados Podados . . . . . . . . . 68

B.3 Gnanadesikan-Kettenring Ortogonalizado . . . . . . . . . . . . . . . . 69

C Programas em R das Simulações $\quad 71$

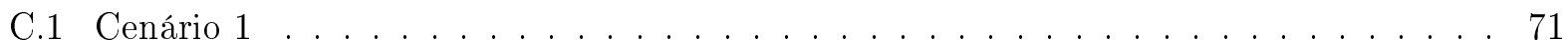

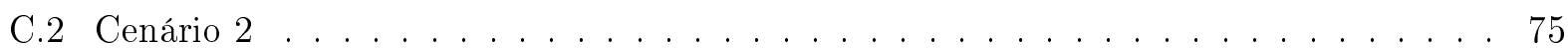

$\begin{array}{ll}\text { Referências Bibliográficas } & 79\end{array}$ 


\section{Lista de Figuras}

2.1 Representação gráfica de um Modelo de Análise de Equações Estruturais. . . . . . . 6

3.1 Representação gráfica de um Modelo de Análise de Regressão. . . . . . . . . . . . . . . 15

3.2 Representação gráfica de um Modelo de Análise Fatorial Exploratória. . . . . . . . . 19

5.1 Modelo de Análise Fatorial Confirmatória para simulação do Cenário 1. . . . . . . . 34

5.2 Modelo de Análise Fatorial Confirmatória para simulação do Cenário 2. . . . . . . . 49 


\section{Lista de Tabelas}

5.1 Esquema para a geração de outliers no Cenário 1. . . . . . . . . . . . . . . 35

5.2 Estatísticas dos mil valores obtidos via simulação para as estimativas dos parâmetros do MEE descrito pela Figura 5.1, por tamanho de amostra e com fração de contaminação zero. . . . . . . . . . . . . . . . . . . 39

5.3 Estatísticas dos mil valores obtidos via simulação para os indicadores clássicos e robustos do MEE descrito pela Figura 5.1, por tamanho de amostra e com fração de contaminação zero. . . . . . . . . . . . . . . . . . . . 40

5.4 Estatísticas dos mil valores obtidos via simulação para as estimativas dos parâmetros do MEE descrito pela Figura 5.1, por tamanho de amostra e com fração de 5\% de contaminação proveniente da lei Exponencial de média unitária. . . . . . . . . . . . 41

5.5 Estatísticas dos mil valores obtidos via simulação para os indicadores clássicos e robustos do MEE descrito pela Figura 5.1, por tamanho de amostra e com fração de $5 \%$ de contaminação proveniente da lei Exponencial de média unitária. . . . . . . . . 42

5.6 Estatísticas dos mil valores obtidos via simulação para as estimativas dos parâmetros do MEE descrito pela Figura 5.1, por tamanho de amostra e com fração de $25 \%$ de contaminação proveniente da lei Exponencial de média unitária. . . . . . . . . . . . 43

5.7 Estatísticas dos mil valores obtidos via simulação para os indicadores clássicos e robustos do MEE descrito pela Figura 5.1, por tamanho de amostra e com fração de 25\% de contaminação proveniente da lei Exponencial de média unitária. . . . . . . . . 44

5.8 Estatísticas dos mil valores obtidos via simulação para as estimativas dos parâmetros do MEE descrito pela Figura 5.1, por tamanho de amostra e com fração de 5\% de contaminação proveniente da lei $t$-Student com cinco graus de liberdade. . . . . . . . 45

5.9 Estatísticas dos mil valores obtidos via simulação para os indicadores clássicos e robustos do MEE descrito pela Figura 5.1, por tamanho de amostra e com fração de $5 \%$ de contaminação proveniente da lei $t$-Student com cinco graus de liberdade. . . . 46

5.10 Estatísticas dos mil valores obtidos via simulação para as estimativas dos parâmetros do MEE descrito pela Figura 5.1, por tamanho de amostra e com fração de $25 \%$ de contaminação proveniente da lei $t$-Student com cinco graus de liberdade. . . . . . . 47

5.11 Estatísticas dos mil valores obtidos via simulação para os indicadores clássicos e robustos do MEE descrito pela Figura 5.1, por tamanho de amostra e com fração de $25 \%$ de contaminação proveniente da lei $t$-Student com cinco graus de liberdade. . . 48

5.12 Esquema para a geração de outliers no Cenário 2 . . . . . . . . . . . . . . . 50 
5.13 Estatísticas dos mil valores obtidos via simulação para as estimativas dos parâmetros do MEE descrito pela Figura 5.2, por tamanho de amostra e com fração de contaminação zero.

5.14 Estatísticas dos mil valores obtidos via simulação para os indicadores clássicos e robustos do MEE descrito pela Figura 5.2, por tamanho de amostra e com fração de contaminação zero. . . . . . . . . . . . . . . . . . . . 5

5.15 Estatísticas dos mil valores obtidos via simulação para as estimativas dos parâmetros do MEE descrito pela Figura 5.2, por tamanho de amostra e com fração de $5 \%$ de contaminação proveniente da lei Log-Normal Padrão. . . . . . . . . . . . . . . . . . . 55

5.16 Estatísticas dos mil valores obtidos via simulação para os indicadores clássicos e robustos do MEE descrito pela Figura 5.2, por tamanho de amostra e com fração de $5 \%$ de contaminação proveniente da lei Log-Normal Padrão. . . . . . . . . . . . . . . 56

5.17 Estatísticas dos mil valores obtidos via simulação para as estimativas dos parâmetros do MEE descrito pela Figura 5.2, por tamanho de amostra e com fração de $25 \%$ de contaminação proveniente da lei Log-Normal Padrão. . . . . . . . . . . . . . . . . . . 57

5.18 Estatísticas dos mil valores obtidos via simulação para os indicadores clássicos e robustos do MEE descrito pela Figura 5.2, por tamanho de amostra e com fração de $25 \%$ de contaminação proveniente da lei Log-Normal Padrão. . . . . . . . . . . . . . 58

5.19 Estatísticas dos mil valores obtidos via simulação para as estimativas dos parâmetros do MEE descrito pela Figura 5.2, por tamanho de amostra e com fração de $5 \%$ de contaminação proveniente da lei Uniforme Contínua em $]-5 ; 5[\ldots \ldots$. . . . . . . 59

5.20 Estatísticas dos mil valores obtidos via simulação para os indicadores clássicos e robustos do MEE descrito pela Figura 5.2, por tamanho de amostra e com fração de $5 \%$ de contaminação proveniente da lei Uniforme Contínua em $]-5 ; 5[$. . . . . . . . 60

5.21 Estatísticas dos mil valores obtidos via simulação para as estimativas dos parâmetros do MEE descrito pela Figura 5.2, por tamanho de amostra e com fração de $25 \%$ de contaminação proveniente da lei Uniforme Contínua em $]-5 ; 5[$. . . . . . . . . . . . 61

5.22 Estatísticas dos mil valores obtidos via simulação para os indicadores clássicos e robustos do MEE descrito pela Figura 5.2, por tamanho de amostra e com fração de $25 \%$ de contaminação proveniente da lei Uniforme Contínua em $]-5 ; 5[\ldots$. . . . . . . 


\section{Capítulo 1}

\section{Introdução}

O Modelo de Equações Estruturais (MEE) é destinado a situações em que o pesquisador parte de um modelo hipotético de relações de interdependência entre variáveis e, com base em um conjunto de dados, visa testar a significância estatística das associações pré-definidas, para então analisar se o modelo que ele propôs é razoável. Seu uso também é indicado quando há variáveis latentes e erros de medida nas variáveis observadas.

De modo geral, a maioria das análises estatísticas passa pelas seguintes etapas: coleta da amostra, descrição dos dados, exploração das relações entre duas ou mais variáveis, formulação de um modelo que descreva as associações identificadas entre as variáveis e obtenção de conclusões com o auxílio de técnicas inferenciais. Sob outra perspectiva, a análise de MEEs inicia com um quadro teórico já formulado pelo pesquisador acerca das possíveis correlações ou relações de causa/efeito entre as variáveis de algum estudo, para então obter uma amostra e averiguar se ela confirma o que foi estabelecido a priori no modelo hipotético.

Por esse diferencial na estratégia de análise, os MEEs vêm ganhando cada vez mais popularidade em diversas áreas do conhecimento, como a Economia, a Educação, a Epidemiologia, o Marketing, a Psicologia e a Sociologia, além da própria Estatística enquanto Ciência Exata.

Devido à capacidade de contemplar relações complexas entre variáveis, essa ferramenta é uma técnica de Análise Multivariada mais geral que outras (e.g., a Análise de Regressão, a Análise Fatorial Exploratória, a Análise Fatorial Confirmatória e a Análise de Correlação Canônica). Contudo, mesmo sendo bastante amplo e versátil, o MEE também possui pressupostos e requer atenção quando os dados amostrais a serem usados não atendem todas as suas assunções.

Assim como ocorre com vários métodos estatísticos, outliers também podem caracterizar um problema bastante grave para MEEs, havendo a necessidade de entender melhor quais são os impactos que eles causam nessa classe de modelos em particular, bem como a maneira de identificá-los e, sobretudo, contorná-los.

Os objetivos desta dissertação consistem em: avaliar o que ocorre com as estimativas dos parâmetros de MEEs ajustados a partir de dados contaminados por outliers, verificar se as tradicionais medidas de diagnóstico sobre o ajuste conseguem detectar esse tipo de problema e averiguar se há benefícios em usar adaptações do Índice da Qualidade do Ajuste (ou GFI, de Goodness-of-Fit Index) e do Índice Corrigido da Qualidade do Ajuste (ou AGFI, de Adjusted Goodness-of-Fit Index) que substituam a usual matriz de covariâncias amostrais ordinárias ou clássicas pelas matrizes de covariâncias amostrais computadas pelos estimadores robustos Gnanadesikan-Kettenring Ortogonalizado, Elipsoide de Volume Mínimo, Covariância de Determinante Mínimo, S e MM. 
Este texto está organizado como segue:

No Capítulo 2, são apresentados os principais fundamentos do MEE, passando pela formulação matemática do modelo, representação gráfica, identificabilidade, estimação, aspectos computacionais e suposições básicas.

O Capítulo 3 revisa conceitos básicos de robustez e apresenta as principais diferenças na aplicação de métodos resistentes a outliers em modelos exploratórios e confirmatórios, dando ênfase aos estimadores robustos estatisticamente mais eficientes e com alto ponto de ruptura.

No Capítulo 4, uma discussão sobre alguns índices da qualidade do ajustamento conhecidos na literatura foi realizada e uma lista de propostas de indicadores construídos a partir de matrizes de covariâncias robustas é apresentada.

Dois estudos de simulação realizados com o fim de avaliar o desempenho dos indicadores vistos no Capítulo 4 quanto à capacidade de detectar o impacto que outliers provocam nas estimativas dos parâmetros de MEEs são exibidos no Capítulo 5. Foram examinados desvios de simetria e excesso de curtose, para diferentes percentuais de contaminação e quantidades de observações, de variáveis observadas perturbadas e de parâmetros a estimar.

Por fim, o Capítulo 6 dedica-se a sumarizar as principais considerações sobre este trabalho e a também sugerir pesquisas futuras. 


\section{Capítulo 2}

\section{Modelos de Equações Estruturais}

O Modelo de Equações Estruturais (MEE) é destinado a situações em que o pesquisador parte de um modelo hipotético de relações de interdependência entre variáveis e, com base em um conjunto de dados, visa testar a significância estatística das associações pré-definidas, para então analisar se o modelo que ele propôs é razoável. Seu uso também é indicado quando há variáveis latentes ou fatores e erros de medida nas variáveis observadas ou manifestas.

Por permitir a inclusão de variáveis latentes, o MEE é uma técnica bastante útil para trabalhar com conceitos abstratos (Lima, 2005a). Além disso, a capacidade de incorporar relações complexas entre variáveis o torna mais geral que várias outras técnicas de Análise Multivariada (e.g., a Análise de Regressão, a Análise Fatorial e a Análise de Correlação Canônica). No entanto, deve-se ter em mente que o objetivo desse tipo de modelagem é confirmatório: seu uso não é adequado para explorar possíveis associações entre variáveis (Pilati e Laros, 2007).

Neste capítulo, serão abordados os seguintes tópicos sobre a análise de modelos de equações estruturais: formulação matemática, representação gráfica, identificabilidade, métodos de estimação, aspectos computacionais e pressupostos da modelagem.

\subsection{Formulação matemática}

Segundo Bollen (1989), a premissa para a modelagem de equações estruturais é a seguinte: se o modelo estivesse correto e se os parâmetros fossem conhecidos, a matriz populacional de covariâncias poderia ser reproduzida com exatidão. Designando por $\boldsymbol{\Sigma}$ a matriz populacional de covariâncias, por $\boldsymbol{\theta}$ um vetor que contém os parâmetros do modelo e por $\boldsymbol{\Sigma}(\boldsymbol{\theta})$ a matriz de covariâncias implícitas escrita como uma função de $\boldsymbol{\theta}$, pode-se expressar aquela afirmação do modo a seguir:

$$
\boldsymbol{\Sigma}=\boldsymbol{\Sigma}(\boldsymbol{\theta})
$$

Seja $\boldsymbol{\eta}=\left[\begin{array}{lll}\eta_{1} & \cdots & \eta_{r}\end{array}\right]^{\prime}$ um vetor de variáveis latentes endógenas relacionado ao vetor de variáveis observadas endógenas ou dependentes $\boldsymbol{y}=\left[\begin{array}{lll}y_{1} & \cdots & y_{p}\end{array}\right]^{\prime}$ através da equação

$$
\boldsymbol{y}=\boldsymbol{\Lambda}_{\boldsymbol{y}} \boldsymbol{\eta}+\varepsilon,
$$

denominada submodelo de mensuração em $\boldsymbol{y}$, em que $\boldsymbol{\Lambda}_{\boldsymbol{y}}=\left[\lambda_{i, j}^{(\boldsymbol{y})}\right]_{p \times r}$ é a matriz dos pesos 
fatoriais de $\boldsymbol{\eta}$ em $\boldsymbol{y}$ e $\boldsymbol{\varepsilon}=\left[\begin{array}{lll}\varepsilon_{1} & \cdots & \varepsilon_{p}\end{array}\right]^{\prime}$ é o vetor dos erros de medida de $\boldsymbol{y}$, com $p, r \in \mathbb{N}$.

Seja $\boldsymbol{\xi}=\left[\begin{array}{lll}\xi_{1} & \cdots & \xi_{s}\end{array}\right]^{\prime}$ um vetor de variáveis latentes exógenas relacionado ao vetor de variáveis observadas exógenas ou independentes $\boldsymbol{x}=\left[\begin{array}{lll}x_{1} & \cdots & x_{q}\end{array}\right]^{\prime}$ a partir da expressão

$$
\boldsymbol{x}=\Lambda_{x} \xi+\delta,
$$

chamada de submodelo de mensuração em $\boldsymbol{x}$, em que $\boldsymbol{\Lambda}_{\boldsymbol{x}}=\left[\lambda_{i, j}^{(\boldsymbol{x})}\right]_{q \times s}$ é a matriz dos pesos fatoriais de $\boldsymbol{\xi}$ em $\boldsymbol{x}$ e $\boldsymbol{\delta}=\left[\begin{array}{lll}\delta_{1} & \cdots & \delta_{q}\end{array}\right]^{\prime}$ é o vetor dos erros de medida de $\boldsymbol{x}$, com $q, s \in \mathbb{N}$.

As equações (2.2) e (2.3) compõem o que se conhece por modelo de medida e especificam as relações entre as variáveis observadas e as variáveis latentes, ao passo que as relações entre os vetores de variáveis latentes $\boldsymbol{\eta}$ e $\boldsymbol{\xi}$ ficam a cargo do modelo linear estrutural, definido como:

$$
\eta=B \eta+\Gamma \xi+\zeta
$$

em que $\boldsymbol{B}=\left[B_{i, j}\right]_{r \times r}$ e $\boldsymbol{\Gamma}=\left[\gamma_{i, j}\right]_{r \times s}$ são as matrizes dos respectivos coeficientes de $\boldsymbol{\eta}$ e $\boldsymbol{\xi}$, enquanto que $\boldsymbol{\zeta}=\left[\begin{array}{lll}\zeta_{1} & \cdots & \zeta_{r}\end{array}\right]^{\prime}$ é um vetor de erros conhecido por disturbance.

O sistema de equações matriciais lineares formado pelas expressões (2.2), (2.3) e (2.4) é chamado de Modelo de Equações Estruturais (MEE) e é dado por:

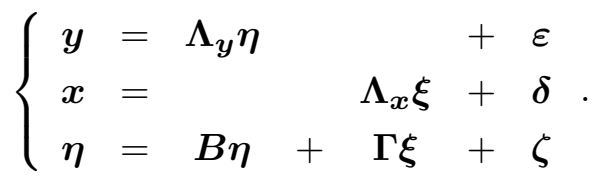

As suposições do MEE são:

- Centragem ${ }^{1}$ das variáveis:

(a) Observadas: $E(\boldsymbol{y})=\mathbf{0}_{p \times 1}$ e $E(\boldsymbol{x})=\mathbf{0}_{q \times 1}$.

(b) Latentes: $E(\boldsymbol{\eta})=\mathbf{0}_{r \times 1}$ e $E(\boldsymbol{\xi})=\mathbf{0}_{s \times 1}$.

(c) De erro: $E(\boldsymbol{\zeta})=\mathbf{0}_{r \times 1}, E(\boldsymbol{\varepsilon})=\mathbf{0}_{p \times 1}$ e $E(\boldsymbol{\delta})=\mathbf{0}_{q \times 1}$.

- Ausência de correlação entre:

(d) $\varepsilon$ e $\boldsymbol{\eta}$.

(e) $\boldsymbol{\delta}$ e $\boldsymbol{\xi}$

(f) $\zeta$ e $\boldsymbol{\xi}$.

(g) $\boldsymbol{\zeta}, \varepsilon$ e $\delta$, mutuamente.

- Ausência de influência direta de uma variável latente endógena sobre ela própria, com:

(h) $B_{1,1}=\cdots=B_{r, r}=0$.

(i) $\operatorname{det}(\boldsymbol{I}-\boldsymbol{B}) \neq 0$, em que $\operatorname{det}(\cdot)$ é o determinante de uma matriz.

\footnotetext{
${ }^{1}$ Seja $\boldsymbol{T}=\left[\begin{array}{lll}T_{1} & \ldots & T_{n}\end{array}\right]$ ' um vetor aleatório real de $n$ variáveis aleatórias reais cujo vetor de médias é dado por $E(\boldsymbol{T})=\left[\begin{array}{lll}E\left(T_{1}\right) & \cdots & E\left(T_{n}\right)\end{array}\right]^{\prime}$. O vetor aleatório real $\boldsymbol{T}-E(\boldsymbol{T})$ é centrado, pois $E(\boldsymbol{T}-E(\boldsymbol{T}))=\mathbf{0}_{n \times 1}$, em que $E(\cdot)$ designa a esperança matemática e $\mathbf{0}_{n \times 1}$ denota um vetor-coluna com $n$ linhas iguais a zero, com $n \in \mathbb{N}$.
} 
Por fim, são adotados alguns símbolos para representar as seguintes matrizes de covariâncias:

$$
\begin{array}{rlrl}
\mathbf{\Phi} & =E\left(\boldsymbol{\xi} \boldsymbol{\xi}^{\prime}\right)=\left[\phi_{i, j}\right]_{s \times s} & \boldsymbol{\Theta}_{\boldsymbol{\varepsilon}}=E\left(\boldsymbol{\varepsilon} \boldsymbol{\varepsilon}^{\prime}\right) & =\left[\theta_{i, j}^{(\boldsymbol{\varepsilon})}\right]_{p \times p} \\
\boldsymbol{\Psi} & =E\left(\boldsymbol{\zeta} \boldsymbol{\zeta}^{\prime}\right)=\left[\psi_{i, j}\right]_{r \times r} & \boldsymbol{\Theta}_{\boldsymbol{\delta}}=E\left(\boldsymbol{\delta} \boldsymbol{\delta}^{\prime}\right)=\left[\theta_{i, j}^{(\boldsymbol{\delta})}\right]_{q \times q}
\end{array}
$$

\subsection{Representação gráfica}

Apesar de ser possível discriminar as relações entre variáveis por meio de equações, tem sido costumeiro representar as associações em diagramas, a partir das convenções arbitradas na chamada Análise de Caminhos. Esse hábito fez com que o MEE se tornasse uma técnica estatística mais acessível a leitores de outras áreas do conhecimento.

Latif (2000) apresenta as regras para as variáveis e flechas, a saber:

- Sobre a inscrição de variáveis:

(a) As variáveis observadas devem estar contidas em retângulos.

(b) As variáveis latentes devem estar inseridas em elipses.

(c) As variáveis de erro devem estar próximas às variáveis observadas/latentes associadas, exteriormente aos retângulos/elipses.

- Sobre a forma e a direção de flechas:

(d) Uma flecha reta de sentido único entre duas variáveis indica influência direta de uma variável sobre a outra, ao passo que uma flecha reta de duplo sentido entre duas variáveis indica uma influência recíproca entre elas, possuindo coeficientes:

1. $\lambda_{i, j}^{(\boldsymbol{x})}$, se vai de $\xi_{j}$ para $x_{i}, \operatorname{com} i \in\{1, \ldots, q\}$ e $j \in\{1, \ldots, s\}$.

2. $\lambda_{i, j}^{(\boldsymbol{y})}$, se vai de $\eta_{j}$ para $y_{i}$, com $i \in\{1, \ldots, p\}$ e $j \in\{1, \ldots, r\}$.

3. $B_{i, j}$, se vai de $\eta_{j}$ para $\eta_{i}$, com $i, j \in\{1, \ldots, r\}, i \neq j$.

4. $\gamma_{i, j}$, se vai de $\xi_{j}$ para $\eta_{i}$, com $i \in\{1, \ldots, r\}$ e $j \in\{1, \ldots, s\}$.

(e) Uma flecha reta de sentido único não pode apontar para $\xi_{i}$, com $i \in\{1, \ldots, s\}$.

(f) Todas as flechas retas de sentido único apontando para $\eta_{j}$ devem provir de $\xi_{i}$ e/ou $\eta_{j^{\prime}}$, com $i \in\{1, \ldots, s\}$ e $j, j^{\prime} \in\{1, \ldots, r\}, j \neq j^{\prime}$.

(g) Uma flecha curva de duplo sentido entre duas variáveis indica que elas podem ser correlacionadas, com coeficientes:

1. $\phi_{i, j}$ ou $\phi_{j, i}$, se entre $\xi_{j}$ e $\xi_{i}$, com $i, j \in\{1, \ldots, s\}, i \neq j$.

2. $\psi_{i, j}$ ou $\psi_{j, i}$, se entre $\zeta_{j}$ e $\zeta_{i}, \operatorname{com} i, j \in\{1, \ldots, r\}, i \neq j$.

3. $\theta_{i, j}^{(\boldsymbol{\delta})}$ ou $\theta_{j, i}^{(\boldsymbol{\delta})}$, se entre $\delta_{j}$ e $\delta_{i}, \operatorname{com} i, j \in\{1, \ldots, q\}, i \neq j$.

4. $\theta_{i, j}^{(\varepsilon)}$ ou $\theta_{j, i}^{(\varepsilon)}$, se entre $\varepsilon_{j}$ e $\varepsilon_{i}, \operatorname{com} i, j \in\{1, \ldots, p\}, i \neq j$.

Como a não existência de uma flecha entre duas variáveis significa que elas não são diretamente relacionadas (embora elas possam estar associadas indiretamente), todas as influências diretas de uma variável em outra devem ser incluídas. Além disso, deve-se ter cuidado com a direção "causal" apontada, pois o ajuste acaba sendo ruim quando o MEE está mal especificado (Sanches, 2005). 
A Figura 2.1 apresenta um exemplo adaptado ${ }^{2}$ de Marôco (2010), podendo-se conferir um MEE com: duas variáveis latentes exógenas correlacionadas, cada uma operacionalizada por três variáveis observadas exógenas; e duas variáveis latentes endógenas reciprocamente influenciadas, cada uma operacionalizada por duas variáveis observadas endógenas. Existe correlação não nula entre duas variáveis de erro associadas a variáveis observadas endógenas e também entre as disturbances. As variáveis latentes exógenas não possuem variáveis de erro em virtude da suposição de ausência de correlação das mesmas com as disturbances.

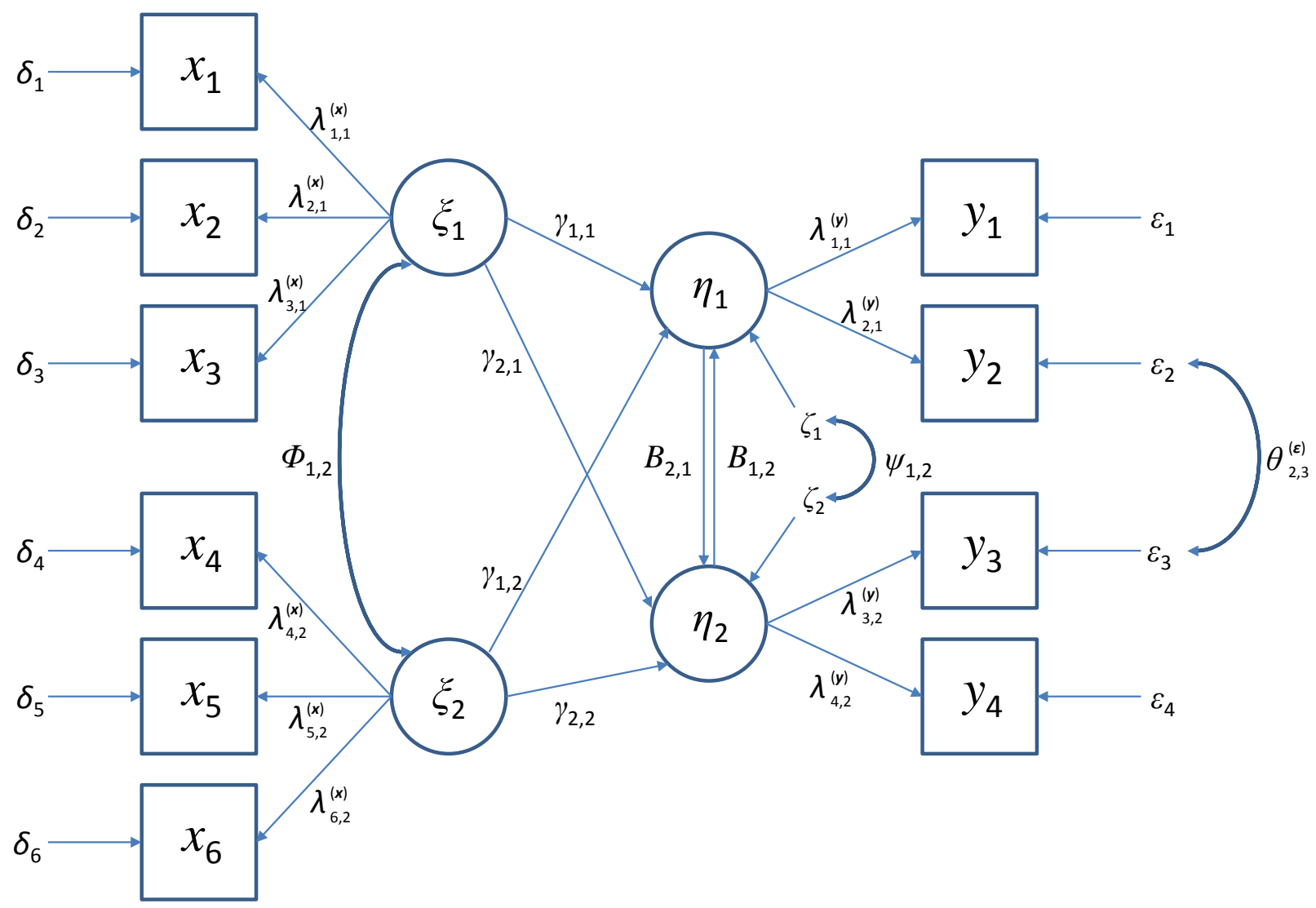

Figura 2.1: Representação gráfica de um Modelo de Análise de Equações Estruturais.

Quanto às correspondentes equações da ilustração acima, tem-se:

- Para o modelo de medida:

$\rightarrow$ Para o submodelo de mensuração em $\boldsymbol{y}:$

$$
\begin{aligned}
& y_{1}=\lambda_{1,1}^{(\boldsymbol{y})} \eta_{1}+\varepsilon_{1} \\
& y_{2}=\lambda_{2,1}^{(\boldsymbol{y})} \eta_{1}+\varepsilon_{2} \\
& y_{3}=\lambda_{3,2}^{(\boldsymbol{y})} \eta_{2}+\varepsilon_{3} \\
& y_{4}=\lambda_{4,2}^{(\boldsymbol{y})} \eta_{2}+\varepsilon_{4}
\end{aligned}
$$

\footnotetext{
${ }^{2} \mathrm{Na}$ figura original, as variáveis de erro estão dentro de elipses.
} 
ou, equivalentemente,

$$
\left[\begin{array}{l}
y_{1} \\
y_{2} \\
y_{3} \\
y_{4}
\end{array}\right]=\left[\begin{array}{cc}
\lambda_{1,1}^{(\boldsymbol{y})} & 0 \\
\lambda_{2,1}^{(\boldsymbol{y})} & 0 \\
0 & \lambda_{3,2}^{(\boldsymbol{y})} \\
0 & \lambda_{4,2}^{(\boldsymbol{y})}
\end{array}\right]\left[\begin{array}{l}
\eta_{1} \\
\eta_{2}
\end{array}\right]+\left[\begin{array}{c}
\varepsilon_{1} \\
\varepsilon_{2} \\
\varepsilon_{3} \\
\varepsilon_{4}
\end{array}\right] .
$$

$\rightarrow$ Para o submodelo de mensuração em $\boldsymbol{x}$ :

$$
\begin{aligned}
& x_{1}=\lambda_{1,1}^{(\boldsymbol{x})} \xi_{1}+\delta_{1} \\
& x_{2}=\lambda_{2,1}^{(\boldsymbol{x})} \xi_{1}+\delta_{2} \\
& x_{3}=\lambda_{3,1}^{(\boldsymbol{x})} \xi_{1}+\delta_{3} \\
& x_{4}=\lambda_{4,2}^{(\boldsymbol{x})} \xi_{2}+\delta_{4} \\
& x_{5}=\lambda_{5,2}^{(\boldsymbol{x})} \xi_{2}+\delta_{5} \\
& x_{6}=\lambda_{6,2}^{(\boldsymbol{x})} \xi_{2}+\delta_{6}
\end{aligned}
$$

ou, similarmente,

$$
\left[\begin{array}{l}
x_{1} \\
x_{2} \\
x_{3} \\
x_{4} \\
x_{5} \\
x_{6}
\end{array}\right]=\left[\begin{array}{cc}
\lambda_{1,1}^{(\boldsymbol{x})} & 0 \\
\lambda_{2,1}^{(\boldsymbol{x})} & 0 \\
\lambda_{3,1}^{(\boldsymbol{x})} & 0 \\
0 & \lambda_{4,2}^{(\boldsymbol{x})} \\
0 & \lambda_{5,2}^{(\boldsymbol{x})} \\
0 & \lambda_{6,2}^{(\boldsymbol{x})}
\end{array}\right]\left[\begin{array}{l}
\xi_{1} \\
\xi_{2}
\end{array}\right]+\left[\begin{array}{c}
\delta_{1} \\
\delta_{2} \\
\delta_{3} \\
\delta_{4} \\
\delta_{5} \\
\delta_{6}
\end{array}\right]
$$

- Para o modelo linear estrutural:

$$
\begin{aligned}
& \eta_{1}=B_{1,2} \eta_{2}+\gamma_{1,1} \xi_{1}+\gamma_{1,2} \xi_{2}+\zeta_{1} \\
& \eta_{2}=B_{2,1} \eta_{1}+\gamma_{2,1} \xi_{1}+\gamma_{2,2} \xi_{2}+\zeta_{2}
\end{aligned}
$$

ou, analogamente,

$$
\left[\begin{array}{l}
\eta_{1} \\
\eta_{2}
\end{array}\right]=\left[\begin{array}{cc}
0 & B_{1,2} \\
B_{2,1} & 0
\end{array}\right]\left[\begin{array}{l}
\eta_{1} \\
\eta_{2}
\end{array}\right]+\left[\begin{array}{ll}
\gamma_{1,1} & \gamma_{1,2} \\
\gamma_{2,1} & \gamma_{2,2}
\end{array}\right]\left[\begin{array}{l}
\xi_{1} \\
\xi_{2}
\end{array}\right]+\left[\begin{array}{l}
\zeta_{1} \\
\zeta_{2}
\end{array}\right]
$$

- Para as matrizes de covariâncias:

$$
\begin{array}{ccc}
\boldsymbol{\Phi}=\left[\begin{array}{cc}
\phi_{1,1} & \phi_{1,2} \\
\phi_{2,1} & \phi_{2,2}
\end{array}\right] & \boldsymbol{\Psi}=\left[\begin{array}{cc}
\psi_{1,1} & \psi_{1,2} \\
\psi_{2,1} & \psi_{2,2}
\end{array}\right] \\
\boldsymbol{\Theta}_{\boldsymbol{\varepsilon}}=\left[\begin{array}{cccc}
\theta_{1,1}^{(\boldsymbol{\varepsilon})} & 0 & 0 & 0 \\
0 & \theta_{2,2}^{(\boldsymbol{\varepsilon})} & \theta_{2,3}^{(\boldsymbol{\varepsilon})} & 0 \\
0 & \theta_{3,2}^{(\boldsymbol{\varepsilon})} & \theta_{3,3}^{(\boldsymbol{\varepsilon})} & 0 \\
0 & 0 & 0 & \theta_{4,4}^{(\boldsymbol{\varepsilon})}
\end{array}\right] & \boldsymbol{\Theta}_{\boldsymbol{\delta}}=\left[\begin{array}{cccccc}
\theta_{1,1}^{(\boldsymbol{\delta})} & 0 & 0 & 0 & 0 & 0 \\
0 & \theta_{2,2}^{(\boldsymbol{\delta})} & 0 & 0 & 0 & 0 \\
0 & 0 & \theta_{3,3}^{(\boldsymbol{\delta})} & 0 & 0 & 0 \\
0 & 0 & 0 & \theta_{4,4}^{(\boldsymbol{\delta})} & 0 & 0 \\
0 & 0 & 0 & 0 & \theta_{5,5}^{(\boldsymbol{\delta})} & 0 \\
0 & 0 & 0 & 0 & 0 & \theta_{6,6}^{(\boldsymbol{\delta})}
\end{array}\right]
\end{array}
$$




\subsection{Identificabilidade}

Um modelo está identificado se for possível derivar uma estimativa única para cada um dos parâmetros de interesse (Lima, 2005b). Alternativamente, um MEE é dito ser identificável quando a seguinte condição é satisfeita:

$$
\boldsymbol{\Sigma}\left(\boldsymbol{\theta}_{1}\right)=\boldsymbol{\Sigma}\left(\boldsymbol{\theta}_{2}\right) \quad \Rightarrow \quad \boldsymbol{\theta}_{1}=\boldsymbol{\theta}_{2}
$$

Quando os parâmetros do modelo são funções que não conduzem a uma solução única, o MEE é dito ser subidentificado; por outro lado, se há excesso de informação sobre os parâmetros, o referido modelo é considerado superidentificado.

Rocha (2010) e Melhado (2009) ilustram a superidentificação e a subidentificação com analogias a sistemas lineares incompatíveis e a sistemas lineares compatíveis e indeterminados, respectivamente. De fato, essas associações fazem sentido, uma vez que a ideia de "informação" tem a ver com graus de liberdade e, portanto, também com a quantidade de elementos não redundantes da matriz $\boldsymbol{\Sigma}(\boldsymbol{\theta})$, não ultrapassando $(p+q)(p+q+1) / 2$ devido à simetria matricial.

Deve-se suspeitar de problemas com a identificabilidade de um MEE quando as estimativas das variâncias de seus parâmetros são muito grandes (Amorim et al., 2010). A existência de multicolinearidade entre variáveis observadas ou a utilização de uma amostra de dimensão baixa também podem ser causadoras desse tipo de avaria, de acordo com Marôco (2010).

Há regras empíricas para detectar defeitos na identificabilidade apresentadas como condições necessárias e/ou suficientes, as quais são aplicadas pela maioria dos softwares de MEE antes de realizar o ajustamento. As mais conhecidas podem ser consultadas em Hair et al. (2010).

\subsection{Métodos de estimação}

Como $\boldsymbol{\Sigma}$ é desconhecida, a hipótese representada pela equação matricial $\boldsymbol{S}=\boldsymbol{\Sigma}(\boldsymbol{\theta})$ é admitida com a finalidade de obter as estimativas dos parâmetros do MEE, sendo $\boldsymbol{S}$ a usual matriz simétrica de covariâncias amostrais das $p+q$ variáveis centradas observadas em $n$ sujeitos, conhecida por matriz de covariâncias ordinárias ou clássicas, com entradas:

$$
\forall i, j \in\{1,2, \ldots, p+q\}, \quad s_{i, j}=\frac{1}{n-1} \sum_{k=1}^{n} z_{i, k} z_{j, k}=\left\{\begin{array}{ll}
s_{i, i} & , \quad i=j \\
s_{j, i} & , \quad i \neq j
\end{array},\right.
$$

em que $s_{i, i}=\sum_{k=1}^{n} z_{i, k}^{2} /(n-1)=\widehat{\operatorname{Var}\left(z_{i}\right)}$ é a variância amostral da $i$-ésima componente centrada do vetor $\boldsymbol{z}=\left[\begin{array}{ll}\boldsymbol{y}^{\prime} & \boldsymbol{x}^{\prime}\end{array}\right]^{\prime}$ de variáveis observadas, com $n \in \mathbb{N}$.

Bollen (1989) mostra que $\boldsymbol{\Sigma}(\boldsymbol{\theta})$ pode ser particionada em blocos que separam a variância de $\boldsymbol{x}$, a variância de $\boldsymbol{y}$ e a covariância entre $\boldsymbol{x}$ e $\boldsymbol{y}$, reescrevendo-a como transformações (não lineares) dos parâmetros do MEE, do seguinte jeito:

$$
\begin{aligned}
\boldsymbol{\Sigma}(\boldsymbol{\theta}) & =\left[\begin{array}{cc}
E\left(\boldsymbol{y} \boldsymbol{y}^{\prime}\right) & E\left(\boldsymbol{x} \boldsymbol{y}^{\prime}\right) \\
E\left(\boldsymbol{y} \boldsymbol{x}^{\prime}\right) & E\left(\boldsymbol{x} \boldsymbol{x}^{\prime}\right)
\end{array}\right] \\
& =\left[\begin{array}{cc}
\boldsymbol{\Lambda}_{\boldsymbol{y}}(\boldsymbol{I}-\boldsymbol{B})^{-1}\left(\boldsymbol{\Gamma} \boldsymbol{\Phi} \boldsymbol{\Gamma}^{\prime}+\boldsymbol{\Psi}\right)\left(\boldsymbol{I}-\boldsymbol{B}^{\prime}\right)^{-1} \boldsymbol{\Lambda}_{\boldsymbol{y}}^{\prime}+\boldsymbol{\Theta}_{\boldsymbol{\epsilon}} & \boldsymbol{\Lambda}_{\boldsymbol{y}}(\boldsymbol{I}-\boldsymbol{B})^{-1} \boldsymbol{\Gamma} \boldsymbol{\Psi} \boldsymbol{\Lambda}_{\boldsymbol{x}}^{\prime} \\
\boldsymbol{\Lambda}_{\boldsymbol{x}} \boldsymbol{\Psi} \boldsymbol{\Gamma}^{\prime}\left(\boldsymbol{I}-\boldsymbol{B}^{\prime}\right)^{-1} \boldsymbol{\Lambda}_{\boldsymbol{y}}^{\prime} & \boldsymbol{\Lambda}_{\boldsymbol{x}} \boldsymbol{\Psi} \boldsymbol{\Lambda}_{\boldsymbol{x}}^{\prime}+\boldsymbol{\Theta}_{\boldsymbol{\delta}}
\end{array}\right]
\end{aligned}
$$


As funções de ajustamento ou discrepância são minimizadas com o objetivo de estimar o vetor de parâmetros $\boldsymbol{\theta}$ e denotadas por $F(\boldsymbol{S}, \boldsymbol{\Sigma}(\boldsymbol{\theta}))$. Conforme Mulaik (2009b), elas são duas vezes diferenciáveis, contínuas em $\boldsymbol{S}$ e $\boldsymbol{\Sigma}(\boldsymbol{\theta})$, não negativas e satisfazem a condição $F(\boldsymbol{S}, \boldsymbol{\Sigma}(\boldsymbol{\theta}))=0$ se, e somente se, $\boldsymbol{S}=\boldsymbol{\Sigma}(\boldsymbol{\theta})$. Fixando-se $\boldsymbol{\theta}, F(\boldsymbol{S}, \boldsymbol{\Sigma}(\boldsymbol{\theta}))$ é um escalar.

Nesse aspecto, o MEE também se distingue de outras técnicas estatísticas, como a Análise de Regressão: ao invés de minimizar funções $\operatorname{dos} k(k \in\{1, \ldots, n\})$ individuais valores observados e valores preditos, é minimizada a diferença entre os pares $(i, j)(i, j \in\{1, \ldots, p+q\})$ de covariâncias amostrais e covariâncias preditas pelo modelo. Lattin et al. (2011) ainda pontuam que a diferença essencial entre a Análise Fatorial Exploratória e a Análise Fatorial Confirmatória é o procedimento para a obtenção das soluções.

Existem várias funções $F$ que podem ser utilizadas para indicar a magnitude das diferenças entre os elementos de $\boldsymbol{S}$ e $\boldsymbol{\Sigma}(\boldsymbol{\theta})$. Nesta seção, serão contempladas as funções de ajustamento construídas pelos métodos de estimação de Mínimos Quadrados Ordinários, de Mínimos Quadrados Generalizados e de Máxima Verossimilhança.

\subsubsection{Mínimos Quadrados Ordinários}

Partindo-se de um critério de Mínimos Quadrados Ordinários (MQO), no sentido de minimizar uma função da soma dos quadrados das diferenças entre $s_{i, j}$ e $\sigma_{i, j}(\boldsymbol{\theta})$, pode-se definir

$$
F_{\mathrm{MQO}}(\boldsymbol{S}, \boldsymbol{\Sigma}(\boldsymbol{\theta}))=\frac{1}{2} \operatorname{tr}\left\{[\boldsymbol{S}-\boldsymbol{\Sigma}(\boldsymbol{\theta})]^{\prime}[\boldsymbol{S}-\boldsymbol{\Sigma}(\boldsymbol{\theta})]\right\}=\frac{1}{2} \operatorname{tr}\left\{[\boldsymbol{S}-\boldsymbol{\Sigma}(\boldsymbol{\theta})]^{2}\right\}
$$

em que $\operatorname{tr}(\cdot)$ designa a função traço e $[\boldsymbol{S}-\boldsymbol{\Sigma}(\boldsymbol{\theta})]^{\prime}=\boldsymbol{S}^{\prime}-\boldsymbol{\Sigma}^{\prime}(\boldsymbol{\theta})=\boldsymbol{S}-\boldsymbol{\Sigma}(\boldsymbol{\theta})$.

Usando as propriedades descritas nas expressões A.3 e A.4, a derivada parcial de (2.12) em relação a uma componente qualquer $\theta$ de $\boldsymbol{\theta}$ é dada por

$$
\frac{\partial}{\partial \theta} F_{\mathrm{MQO}}(\boldsymbol{S}, \boldsymbol{\Sigma}(\boldsymbol{\theta}))=\frac{1}{2} \operatorname{tr}\left\{2[\boldsymbol{S}-\boldsymbol{\Sigma}(\boldsymbol{\theta})] \frac{\partial}{\partial \theta}[\boldsymbol{S}-\boldsymbol{\Sigma}(\boldsymbol{\theta})]\right\}=\operatorname{tr}\left\{[\boldsymbol{\Sigma}(\boldsymbol{\theta})-\boldsymbol{S}] \frac{\partial}{\partial \theta} \boldsymbol{\Sigma}(\boldsymbol{\theta})\right\},
$$

uma vez que a derivada parcial de $\boldsymbol{S}$ em relação a $\theta$ é igual à matriz quadrada nula de ordem $p+q$.

De modo análogo à expressão (2.13), decorre que a derivada parcial de (2.12) em relação a $\theta_{u} \mathrm{e}$ $\theta_{v}$, duas componentes quaisquer de $\boldsymbol{\theta}$, possui a seguinte forma:

$$
\frac{\partial^{2}}{\partial \theta_{u} \partial \theta_{v}} F_{\mathrm{MQO}}(\boldsymbol{S}, \boldsymbol{\Sigma}(\boldsymbol{\theta}))=\operatorname{tr}\left\{\left[\frac{\partial}{\partial \theta_{u}} \boldsymbol{\Sigma}(\boldsymbol{\theta})\right] \cdot\left[\frac{\partial}{\partial \theta_{v}} \boldsymbol{\Sigma}(\boldsymbol{\theta})\right]\right\}
$$

\subsubsection{Mínimos Quadrados Generalizados}

Inserindo à expressão (2.12) uma matriz de pesos $\boldsymbol{W} \neq \boldsymbol{I}$ quadrada de ordem $p+q$, não singular e convergente em probabilidade a uma matriz positiva-definida ${ }^{3}$, obtém-se a função de discrepância de Mínimos Quadrados Generalizados (MQG), dada por:

$$
F_{\mathrm{MQG}}(\boldsymbol{S}, \boldsymbol{\Sigma}(\boldsymbol{\theta}))=\frac{1}{2} \operatorname{tr}\left\{[\boldsymbol{S}-\boldsymbol{\Sigma}(\boldsymbol{\theta})] \boldsymbol{W}^{-1}\right\}^{2} .
$$

A opção mais usual é a escolha $\boldsymbol{W}^{-1}=\boldsymbol{S}^{-1}$ como um estimador consistente para $\boldsymbol{\Sigma}^{-1}$, podendo-

\footnotetext{
${ }^{3}$ Esse tópico de convergência pode ser conferido e aprofundado em Leite e Singer (1990).
} 
se reescrever a expressão (2.15) do jeito seguinte:

$$
F_{\mathrm{MQG}}(\boldsymbol{S}, \boldsymbol{\Sigma}(\boldsymbol{\theta}))=\frac{1}{2} \operatorname{tr}\left\{[\boldsymbol{S}-\boldsymbol{\Sigma}(\boldsymbol{\theta})] \boldsymbol{S}^{-1}\right\}^{2} .
$$

Usando as propriedades descritas nas expressões A.3 e A.4, a derivada parcial de (2.16) em relação a uma componente qualquer $\theta$ de $\boldsymbol{\theta}$ é dada por

$$
\begin{aligned}
\frac{\partial}{\partial \theta} F_{\mathrm{MQG}}(\boldsymbol{S}, \boldsymbol{\Sigma}(\boldsymbol{\theta})) & =\frac{1}{2} \operatorname{tr}\left\{2[\boldsymbol{S}-\boldsymbol{\Sigma}(\boldsymbol{\theta})] \boldsymbol{S}^{-1} \frac{\partial}{\partial \theta}[\boldsymbol{S}-\boldsymbol{\Sigma}(\boldsymbol{\theta})] \boldsymbol{S}^{-1}\right\} \\
& =\operatorname{tr}\left\{\boldsymbol{S}^{-1}[\boldsymbol{\Sigma}(\boldsymbol{\theta})-\boldsymbol{S}] \boldsymbol{S}^{-1} \frac{\partial}{\partial \theta} \boldsymbol{\Sigma}(\boldsymbol{\theta})\right\}
\end{aligned}
$$

uma vez que a derivada parcial de $\boldsymbol{S}$ em relação a $\theta$ é igual à matriz quadrada nula de ordem $p+q$.

De modo análogo à expressão (2.17), decorre que a derivada parcial de (2.16) em relação a $\theta_{u} \mathrm{e}$ $\theta_{v}$, duas componentes quaisquer de $\boldsymbol{\theta}$, possui a seguinte forma:

$$
\frac{\partial^{2}}{\partial \theta_{u} \partial \theta_{v}} F_{\mathrm{MQG}}(\boldsymbol{S}, \boldsymbol{\Sigma}(\boldsymbol{\theta}))=\operatorname{tr}\left\{\left[\boldsymbol{S}^{-1} \frac{\partial}{\partial \theta_{u}} \boldsymbol{\Sigma}(\boldsymbol{\theta})\right] \cdot\left[\boldsymbol{S}^{-1} \frac{\partial}{\partial \theta_{v}} \boldsymbol{\Sigma}(\boldsymbol{\theta})\right]\right\} .
$$

\subsubsection{Máxima Verossimilhança}

Baseada na estatística do teste da razão de verossimilhanças construído para o ensaio de hipóteses $\mathcal{H}_{0}: \boldsymbol{\Sigma}=\boldsymbol{\Sigma}(\boldsymbol{\theta})$ contra $\mathcal{H}_{1}: \boldsymbol{\Sigma} \neq \boldsymbol{\Sigma}(\boldsymbol{\theta})$ sob a assunção de que $\boldsymbol{z}$ é normalmente distribuído ${ }^{4}$, a função de ajustamento de Máxima Verossimilhança (MV) é definida como:

$$
F_{\mathrm{MV}}(\boldsymbol{S}, \boldsymbol{\Sigma}(\boldsymbol{\theta}))=\ln \operatorname{det} \boldsymbol{\Sigma}(\boldsymbol{\theta})-\ln \operatorname{det} \boldsymbol{S}+\operatorname{tr}\left(\boldsymbol{S} \boldsymbol{\Sigma}^{-1}(\boldsymbol{\theta})\right)-(p+q)
$$

A derivada parcial de (2.19) em relação a uma componente $\theta$ de $\boldsymbol{\theta}$ é:

$$
\begin{aligned}
\frac{\partial}{\partial \theta} F_{\mathrm{MV}}(\boldsymbol{S}, \boldsymbol{\Sigma}(\boldsymbol{\theta})) & =\operatorname{tr}\left[\boldsymbol{\Sigma}^{-1}(\boldsymbol{\theta}) \frac{\partial}{\partial \theta} \boldsymbol{\Sigma}(\boldsymbol{\theta})\right]-\operatorname{tr}\left[\boldsymbol{\Sigma}^{-1}(\boldsymbol{\theta}) \boldsymbol{S} \boldsymbol{\Sigma}^{-1}(\boldsymbol{\theta}) \frac{\partial}{\partial \theta} \boldsymbol{\Sigma}(\boldsymbol{\theta})\right] \\
& =\operatorname{tr}\left\{\left[\boldsymbol{\Sigma}^{-1}(\boldsymbol{\theta})-\boldsymbol{\Sigma}^{-1}(\boldsymbol{\theta}) \boldsymbol{S} \boldsymbol{\Sigma}^{-1}(\boldsymbol{\theta})\right] \frac{\partial}{\partial \theta} \boldsymbol{\Sigma}(\boldsymbol{\theta})\right\} \\
& =\operatorname{tr}\left\{\left[\boldsymbol{I}-\boldsymbol{\Sigma}^{-1}(\boldsymbol{\theta}) \boldsymbol{S}\right] \boldsymbol{\Sigma}^{-1}(\boldsymbol{\theta}) \frac{\partial}{\partial \theta} \boldsymbol{\Sigma}(\boldsymbol{\theta})\right\} \\
& =\operatorname{tr}\left\{\boldsymbol{\Sigma}^{-1}(\boldsymbol{\theta})[\boldsymbol{\Sigma}(\boldsymbol{\theta})-\boldsymbol{S}] \boldsymbol{\Sigma}^{-1}(\boldsymbol{\theta}) \frac{\partial}{\partial \theta} \boldsymbol{\Sigma}(\boldsymbol{\theta})\right\},
\end{aligned}
$$

em que se fez uso das propriedades descritas nas expressões A.6 e A.7.

Procedendo de modo similar ao que foi feito para chegar ao resultado (2.20), a derivada parcial de (2.19) em relação a $\theta_{u}$ e $\theta_{v}$, duas quaisquer componentes de $\boldsymbol{\theta}$, é:

$$
\frac{\partial^{2}}{\partial \theta_{u} \partial \theta_{v}} F_{\mathrm{MV}}(\boldsymbol{S}, \boldsymbol{\Sigma}(\boldsymbol{\theta}))=\operatorname{tr}\left\{\left[\boldsymbol{\Sigma}^{-1}(\boldsymbol{\theta}) \frac{\partial}{\partial \theta_{u}} \boldsymbol{\Sigma}(\boldsymbol{\theta})\right] \cdot\left[\boldsymbol{\Sigma}^{-1}(\boldsymbol{\theta}) \frac{\partial}{\partial \theta_{v}} \boldsymbol{\Sigma}(\boldsymbol{\theta})\right]\right\} .
$$

\footnotetext{
${ }^{4}$ Detalhes podem ser encontrados em Bollen (1989) e Ribeiro (2010), havendo apenas a necessidade de manipular as expressões fornecidas para a estatística de teste, de modo que elas zerem quando $\boldsymbol{S}=\boldsymbol{\Sigma}(\boldsymbol{\theta})$.
} 


\subsection{Aspectos computacionais}

Independentemente da função $F$ escolhida, o método iterativo consiste em obter recursivamente estimativas $\boldsymbol{\theta}^{(1)}, \boldsymbol{\theta}^{(2)}, \ldots$ de $\boldsymbol{\theta}$, tais que

$$
F\left(\boldsymbol{S}, \boldsymbol{\Sigma}\left(\boldsymbol{\theta}^{(\kappa+1)}\right)\right)<F\left(\boldsymbol{S}, \boldsymbol{\Sigma}\left(\boldsymbol{\theta}^{(\kappa)}\right)\right)
$$

e

$$
\left\|\boldsymbol{\theta}^{(\kappa+1)}-\boldsymbol{\theta}^{(\kappa)}\right\|<\epsilon
$$

para um "pequeno" erro numérico $\epsilon>0$ arbitrariamente fixado como critério de parada, com $\kappa \in \mathbb{N}$.

$\mathrm{O}$ algoritmo que tem sido usado para minimizar $F$ usualmente apresenta a forma:

$$
\boldsymbol{\theta}^{(\kappa+1)}=\boldsymbol{\theta}^{(\kappa)}+\alpha_{\kappa}\left[\boldsymbol{H}_{\kappa}\left(\boldsymbol{\theta}^{(\kappa)}\right)\right]^{-1} \boldsymbol{g}_{\kappa}\left(\boldsymbol{\theta}^{(\kappa)}\right),
$$

em que $\boldsymbol{\theta}^{(\kappa)}$ é a estimativa do vetor de parâmetros da $\kappa$-ésima iteração, $\left.\left.\alpha_{\kappa} \in\right] 0 ; 1\right]$ é um "tamanho de passo" da $\kappa$-ésima iteração $\left(\alpha_{\kappa}=1\right.$, em geral $), \boldsymbol{H}_{\kappa}\left(\boldsymbol{\theta}^{(\kappa)}\right)$ é uma aproximação na $\kappa$-ésima iteração à matriz hessiana contendo as segundas derivadas de $F$ em relação aos parâmetros do modelo e $\boldsymbol{g}_{\kappa}\left(\boldsymbol{\theta}^{\kappa}\right)$ é o gradiente negativo de $F$ avaliado em $\boldsymbol{\theta}^{(\kappa)}, \operatorname{com} \kappa \in \mathbb{N}$.

As derivadas de primeira e segunda ordem das funções de discrepância apresentadas nas Subseções 2.4.1, 2.4.2 e 2.4.3 podem ser genericamente reescritas do seguinte modo:

$$
\frac{\partial}{\partial \theta} F(\boldsymbol{S}, \boldsymbol{\Sigma}(\boldsymbol{\theta}))=\operatorname{tr}\left\{\boldsymbol{A}[\boldsymbol{\Sigma}(\boldsymbol{\theta})-\boldsymbol{S}] \boldsymbol{A} \frac{\partial}{\partial \theta} \boldsymbol{\Sigma}(\boldsymbol{\theta})\right\}
$$

$\mathrm{e}$

$$
\frac{\partial^{2}}{\partial \theta_{i} \partial \theta_{j}} F(\boldsymbol{S}, \boldsymbol{\Sigma}(\boldsymbol{\theta}))=\operatorname{tr}\left\{\left[\boldsymbol{A} \frac{\partial}{\partial \theta_{i}} \boldsymbol{\Sigma}(\boldsymbol{\theta})\right] \cdot\left[\boldsymbol{A} \frac{\partial}{\partial \theta_{j}} \boldsymbol{\Sigma}(\boldsymbol{\theta})\right]\right\},
$$

em que $\boldsymbol{A}=\boldsymbol{I}$ para Mínimos Quadrados Ordinários, $\boldsymbol{A}=\boldsymbol{S}^{-1}$ para Mínimos Quadrados Generalizados e $\boldsymbol{A}=\boldsymbol{\Sigma}^{-1}(\boldsymbol{\theta})$ para Máxima Verossimilhança.

Everitt (1984) comenta que o uso de (2.25) e (2.26) diretamente em (2.24) conduz ao algoritmo de Newton-Raphson, destacando que a dificuldade desse procedimento é que a matriz hessiana costuma ser complicada de operar, impactando no tempo de processamento de cada iteração.

Amostras "pequenas", problemas de especificação do modelo e variáveis observadas com outliers, assimetrias extremas ou variâncias e covariâncias muito heterogêneas podem ser responsáveis pela não convergência de um processo iterativo (Marôco, 2010).

Alguns programas de computador usados para ajustar MEEs são: AMOS/SPSS (Blunch, 2008), Mplus (Muthén e Muthén, 1998-2010), R (R Core Team, 2012) e SAS (Hatcher, 1996).

\subsection{Pressupostos da modelagem}

De modo bastante sistemático, Marôco (2010) alerta que a ocorrência de certas situações comuns na prática podem comprometer os resultados das análises, devendo-se garantir que os seguintes pontos estejam atendidos: 
- Variáveis observadas:

- Escala e distribuição: a escala das variáveis observadas deve estar pelo menos no nível intervalar, sendo desejável ter a continuidade acompanhada da normalidade multivariada.

- Associação: as covariâncias amostrais entre as variáveis observadas devem ser não nulas, para que os submodelos de mensuração do modelo de medida do MEE sejam relevantes.

- Outliers: os dados não devem estar contaminados por valores atípicos.

- Variáveis latentes:

- Indicadores múltiplos: as variáveis latentes devem possuir fiabilidades elevadas ${ }^{5}$ e cada uma delas deve ser "operacionalizada" por três ou mais variáveis observadas.

- Linearidade: as variáveis latentes devem estar linearmente relacionadas entre si (pelo modelo linear estrutural) e também com as variáveis observadas (pelos submodelos de mensuração do modelo de medida), para que se justifique o uso do MEE.

- Amostra aleatória: deve-se ter independência entre as observações de sujeitos distintos.

- Ausência de multicolinearidade/forte correlação entre variáveis independentes.

Algumas dissertações e teses deste programa de pós-graduação já avaliaram o que ocorre quando alguns dos pressupostos acima mencionados não são atendidos, como também consideraram outros tipos de violação. Sanches (2005) estudou o impacto de especificações equivocadas sobre a direção das relações de influência entre variáveis (i.e., o efeito da troca do sentido das flechas) nas estimativas dos parâmetros. Bistaffa (2011) contemplou a situação de se ter variáveis observadas categóricas ordinais, sugerindo o uso de algumas matrizes de correlação especiais. Melhado (2009) apresentou resultados teóricos sobre pequenas perturbações provenientes de observações influentes ${ }^{6}$ no MEE com distribuição elíptica.

Nos próximos capítulos, esta dissertação analisará o impacto provocado pelo uso de dados perturbados por outliers nas estimativas dos parâmetros de um MEE, bem como estudará algumas medidas adequadas para diagnosticar esse tipo de falha.

\footnotetext{
${ }^{5}$ Em Hill e Hill (2005), podem ser conferidas algumas recomendações sobre o tratamento e a avaliação da confiabilidade de variáveis não mensuráveis porém aproximáveis a partir de variáveis que podem ser observadas, costumeiramente relacionadas a variáveis latentes e a dados oriundos de pesquisas por questionário.

${ }^{6}$ Segundo Paula (2013), observações influentes são aqueles pontos amostrais que exercem um peso desproporcional nas estimativas dos parâmetros de um modelo, sendo localmente influentes quando são responsáveis por "pequenas" perturbações no modelo.
} 


\section{Capítulo 3}

\section{Análise de Outliers}

Segundo Yuan e Bentler (2001), outliers perturbam as estimativas dos parâmetros de MEEs ajustados a partir do método de Máxima Verossimilhança, uma vez que a violação do pressuposto de normalidade multivariada distorce $\boldsymbol{S}$, afetando $F_{\mathrm{MV}}(\boldsymbol{S}, \boldsymbol{\Sigma}(\boldsymbol{\theta}))$ e, por fim, $\hat{\boldsymbol{\theta}}_{\mathrm{MV}}$.

$\mathrm{Na}$ prática, torna-se necessário saber se as estimativas obtidas estão contaminadas e, em caso afirmativo, também conhecer maneiras de contornar esse problema, devendo-se recorrer à análise de resíduos e ao uso de métodos robustos.

Neste capítulo, serão discutidos os resíduos em MEEs, alguns conceitos da área de robustez e as diferenças na aplicação de métodos robustos em modelos exploratórios e confirmatórios, com ênfase nos métodos de computação de matrizes de covariâncias que combinem alto ponto de ruptura e alta eficiência assintótica sob normalidade em distribuição.

\subsection{Resíduos}

Recordando que a hipótese sobre a estrutura de covariância para a modelagem é $\mathcal{H}_{0}: \boldsymbol{\Sigma}=\boldsymbol{\Sigma}(\boldsymbol{\theta})$, é imediato que ela é verificada verdadeira quando $\boldsymbol{\Sigma}-\boldsymbol{\Sigma}(\boldsymbol{\theta})=0$.

Como as matrizes $\boldsymbol{\Sigma}$ e $\boldsymbol{\Sigma}(\boldsymbol{\theta})$ são quantidades populacionais desconhecidas, uma forma de avaliar $\mathcal{H}_{0}$ é a partir da matriz $\boldsymbol{S}-\boldsymbol{\Sigma}(\widehat{\boldsymbol{\theta}})$, denominada matriz de resíduos, em que $\boldsymbol{S}$ é a matriz de covariâncias amostrais de $\boldsymbol{z}=\left[\begin{array}{ll}\boldsymbol{y}^{\prime} & \boldsymbol{x}^{\prime}\end{array}\right]^{\prime}$ e $\boldsymbol{\Sigma}(\widehat{\boldsymbol{\theta}})$ é a matriz de covariâncias $\boldsymbol{\Sigma}(\boldsymbol{\theta})$ avaliada no ponto $\widehat{\boldsymbol{\theta}}$ que minimiza a função de discrepância $F$ escolhida.

Se algum resíduo bruto $s_{i, j}-\sigma_{i, j}(\widehat{\boldsymbol{\theta}})$ de $\boldsymbol{S}-\boldsymbol{\Sigma}(\widehat{\boldsymbol{\theta}})$ é não nulo, significa que o modelo foi mal ajustado (Bistaffa, 2011). No entanto, os resíduos brutos podem variar muito em virtude de a covariância ser uma função bastante sensível à escala das variáveis e ao tamanho da amostra, havendo a necessidade de padronizá-los e, assim, mensurar adequadamente o quanto eles se distanciam de zero. Para contornar este fato, os resíduos estandardizados ou padronizados do MEE foram então apresentados por Bollen (1989) e são dados por:

$$
\forall i, j \in\{1, \ldots, p+q\}, \quad r_{i, j}=\frac{s_{i, j}-\sigma_{i, j}(\widehat{\boldsymbol{\theta}})}{\sqrt{\left[\sigma_{i, i}(\widehat{\boldsymbol{\theta}}) \sigma_{j, j}(\widehat{\boldsymbol{\theta}})+\sigma_{i, j}(\widehat{\boldsymbol{\theta}})\right] / n}},
$$

os quais assintoticamente seguem a lei Normal Padrão, apontando má especificação do modelo quando muitos de seus valores absolutos são maiores que dois ou três. 
Latif (2000) sugere construir um gráfico quantil-quantil gaussiano ${ }^{1}$ com os resíduos estandardizados. Essa figura indica que o ajuste é satisfatório quando se tem uma nuvem de pontos acima da primeira bissetriz.

\subsection{Conceitos básicos de robustez}

De acordo com Barnett e Lewis (1984), um outlier é uma observação inconsistente com os dados. Uma unidade amostral é um outlier de regressão se for destacada na direção das variáveis observadas endógenas, ao passo que é chamada de ponto de alavanca quando se destaca na direção das variáveis observadas exógenas (Mendes, 1999).

Supondo que $\boldsymbol{z}=\left[\begin{array}{ll}\boldsymbol{y}^{\prime} & \boldsymbol{x}^{\prime}\end{array}\right]^{\prime}$ possui distribuição $G$, formada com probabilidade $1-\alpha$ pela distribuição $F$ e contaminada com probabilidade $\alpha$ por uma distribuição $H(\alpha \in[0 ; 1])$, pode-se escrever essa mistura de distribuições como:

$$
G=(1-\alpha) F+\alpha H
$$

Para um dado estimador $T(G)$ e o caso de $H$ ser particularizada à distribuição $\Delta_{\omega}$, degenerada ${ }^{2}$ em um ponto contaminado $\omega$, tem-se a função de influência (ou IF, de influence function)

$$
\mathrm{IF}=\lim _{\alpha \rightarrow 0} \frac{T\left((1-\alpha) F+\alpha \Delta_{\omega}\right)-T(F)}{\alpha},
$$

definida nos pontos em que o limite existe, permitindo medir o efeito que uma "pequena" fração de contaminação por outliers provoca no estimador (Conceição, 2004).

O ponto de ruptura de um estimador $T$ para uma amostra com $n$ elementos é dado por $\frac{m}{n}$, em que $m$ é o maior número de pontos que podem ser substituídos por valores arbitrários sem que os parâmetros deixem de ser limitados (Hoaglin et al., 1992). Em outras palavras, ele quantifica qual seria a maior porcentagem de contaminação que um estimador poderia suportar e ainda assim fornecer informação confiável sobre o parâmetro (Rousseeuw e Leroy, 2003).

Chamando $D^{m}$ o conjunto de amostras contaminadas $\boldsymbol{z}^{m}$, Conceição (2004) apresenta a seguinte expressão ao ponto de ruptura para amostras finitas:

$$
\epsilon_{n}^{*}=\min _{0 \leq m \leq n}\left(\left\{\frac{m}{n}: \sup _{\boldsymbol{z}^{m} \in D^{m}}\left\|T\left(\boldsymbol{z}^{m}\right)-T(\boldsymbol{z})\right\|=+\infty, m \in\{0, \ldots, n\}\right\} \cup\{1\}\right),
$$

pontuando que a operação de união é para assegurar que o conjunto seja não vazio.

\subsection{Estimadores robustos}

As subseções a seguir apresentarão métodos robustos de estimação para abordagens estatísticas exploratórias (minimização dos erros dos $k$ valores individuais) e confirmatórias (minimização dos erros dos pares $(i, j)$ de covariâncias), com $k \in\{1, \ldots, n\},(i, j) \in\{1, \ldots, p+q\}^{2}$.

Para a abordagem exploratória, os métodos de estimação de Mínimos Quadrados Ordinários,

\footnotetext{
${ }^{1}$ Gráfico quantil-quantil gaussiano é tradução oficial para Gaussian QQ-Plot (Paulino et al., 2011).

${ }^{2}$ A variável aleatória real $W$ segue a lei Dirac ou está degenerada na constante $\omega$, se $\operatorname{Pr}(\{s \in \Omega: W(s)=x\})=$ $\mathbb{1}_{\{\omega\}}(x)$, em que $\mathbb{1}_{A}(\cdot)$ é a função indicadora de um conjunto $A \subseteq \mathbb{R}$, para $x \in \mathbb{R}$ (Gonçalves e Mendes-Lopes, 2000).
} 
M, Menor Mediana dos Quadrados, Mínimos Quadrados Podados, S e MM serão discutidos no contexto da Análise de Regressão e da Análise Fatorial Exploratória.

Na visão confirmatória, os métodos de estimação de Gnanadesikan-Kettenring Ortogonalizado, Elipsoide de Volume Mínimo, Covariância do Determinante Mínimo, S e MM serão revisados, por serem alternativas menos sensíveis a outliers, se comparadas à usual matriz de covariâncias clássicas.

Os algoritmos de alguns dos estimadores robustos acima mencionados estão no Apêndice B.

\subsubsection{Abordagem exploratória}

A Análise de Regressão propõe um modelo estatístico para o relacionamento linear de $q$ variáveis observadas exógenas com uma variável observada endógena, admitindo-se que essas $q+1$ variáveis são centradas e mensuráveis no mesmo conjunto de $n$ indivíduos independentes, a partir da expressão

$$
\forall k\{1, \ldots, n\}, \quad y_{k}=\beta_{1} x_{k, 1}+\beta_{2} x_{k, 2}+\cdots+\beta_{q} x_{k, q}+\varepsilon_{k},
$$

em que $\beta_{1}, \beta_{2}, \ldots, \beta_{q}$ são os $q$ parâmetros lineares a serem estimados, $y_{k}$ é a $k$-ésima observação de uma variável endógena $y=\left[\begin{array}{lll}y_{1} & \cdots & y_{n}\end{array}\right]^{\prime}$ de $\boldsymbol{y}, x_{k, i}$ é a $k$-ésima observação de uma das $q$ variáveis exógenas não colineares $x_{i}=\left[\begin{array}{lll}x_{1, i} & \cdots & x_{n, i}\end{array}\right]^{\prime}$ de $\boldsymbol{x}(i \in\{1,2, \ldots, q\})$ e $\varepsilon_{k}$ é a $k$-ésima observação de uma variável aleatória de erros centrados, homocedásticos, gaussianos e mutuamente não correlacionados $\varepsilon=\left[\begin{array}{lll}\varepsilon_{1} & \cdots & \varepsilon_{n}\end{array}\right]^{\prime}$ de $\varepsilon$.

A Figura 3.1 mostra uma adaptação ${ }^{3}$ da sugestão de Marôco (2010) para representar graficamente o modelo estatístico descrito pela expressão (3.5). Como as variáveis observadas exógenas são fixas na Análise de Regressão, não há variáveis de erro relacionadas a elas. A inexistência de um termo independente, digamos $\beta_{0}$, é decorrente do pressuposto de centragem.

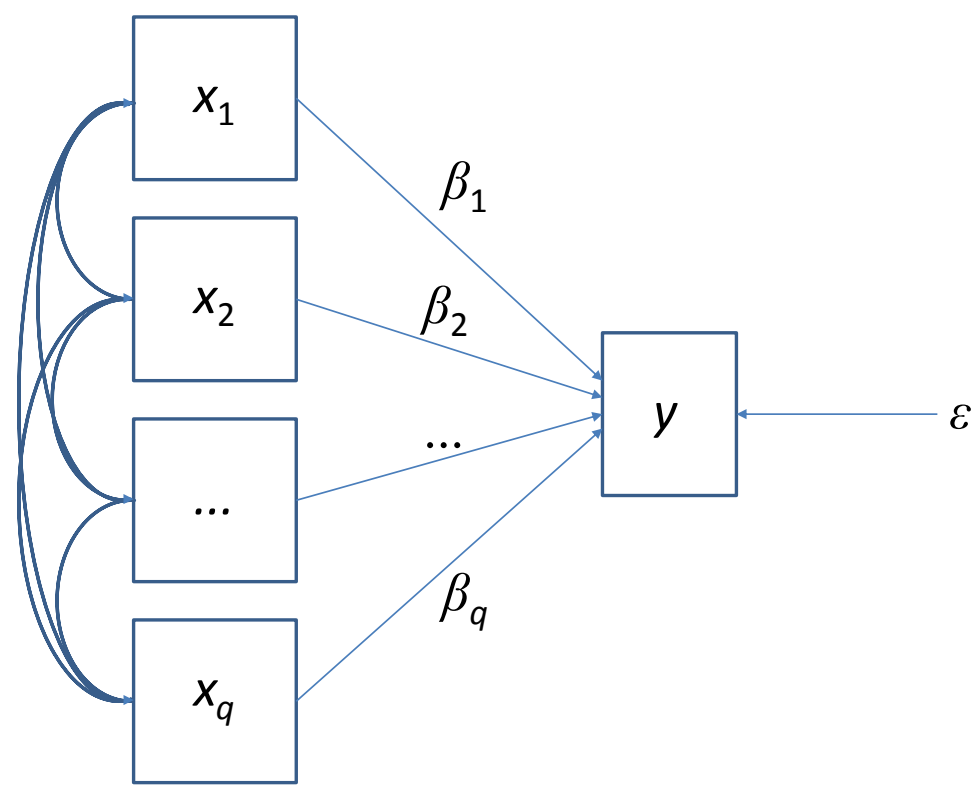

Figura 3.1: Representação gráfica de um Modelo de Análise de Regressão.

\footnotetext{
${ }^{3} \mathrm{Na}$ figura original, a variável de erro está dentro de uma elipse.
} 
Segundo Bouroche e Saporta (1980), deve-se encontrar os $q$ escalares ou estimativas $\widehat{\beta}_{1}, \ldots, \widehat{\beta}_{q}$ da combinação linear

$$
\forall k \in\{1, \ldots, n\}, \quad \xi_{k}=\widehat{\beta}_{1} x_{k, 1}+\widehat{\beta}_{2} x_{k, 2}+\cdots+\widehat{\beta}_{q} x_{k, q},
$$

tal que $\xi$ seja a variável mais próxima possível de $y$ no sentido da distância no subespaço vetorial

$$
V=\left\{\xi \in \mathbb{R}^{n \times 1}: \xi=X \widehat{\beta}\right\}
$$

chamada de valor predito de $y$ ou ainda projeção ortogonal de $y$ em $V$, em que $\xi=\left[\begin{array}{lll}\xi_{1} & \cdots & \xi_{n}\end{array}\right]^{\prime}$, $\widehat{\beta}=\left[\begin{array}{lll}\widehat{\beta}_{1} & \cdots & \widehat{\beta}_{q}\end{array}\right]^{\prime}$ é um vetor de estimativas de $\beta=\left[\begin{array}{lll}\beta_{1} & \cdots & \beta_{q}\end{array}\right]^{\prime}$ e

$$
X=\left[\begin{array}{cccc}
x_{1,1} & x_{1,2} & \cdots & x_{1, q} \\
x_{2,1} & x_{2,2} & \cdots & x_{2, q} \\
\vdots & \vdots & \ddots & \vdots \\
x_{n, 1} & x_{n, 2} & \cdots & x_{n, q}
\end{array}\right]
$$

A obtenção das estimativas $\widehat{\beta}_{1}, \widehat{\beta}_{2}, \ldots, \widehat{\beta}_{q}$ é geralmente alcançada pelo método de Mínimos Quadrados Ordinários, consistindo em minimizar o quadrado da norma euclidiana da variável de erros $\varepsilon$ em relação ao vetor de parâmetros $\beta$ :

$$
\min _{\beta \in \mathbb{R}^{q \times 1}}\|\varepsilon\|^{2}=\min _{\beta \in \mathbb{R}^{q \times 1}}\|y-X \beta\|^{2}=\min _{\beta_{1}, \ldots, \beta_{q} \in \mathbb{R}} \sum_{k=1}^{n}\left[y_{k}-\left(\beta_{1} x_{k, 1}+\beta_{2} x_{k, 2}+\cdots-\beta_{q} x_{k, q}\right)\right]^{2},
$$

cuja solução analítica é

$$
\hat{\beta}=\left(X^{\prime} X\right)^{-1} X^{\prime} y
$$

O método de Mínimos Quadrados Ordinários é bastante sensível à presença de outliers, com ponto de ruptura igual a $1 / n$, o que significa que a existência de um único ponto amostral contaminado pode comprometer a qualidade das estimativas (Rousseeuw e Leroy, 2003). Para contornar esse problema, sugere-se o uso de métodos resistentes à presença de outliers para estimar $\beta$, nomeadamente estimadores robustos.

Para obter um vetor $\hat{\beta}$ de estimativas robustas a outliers em $y$ para as componentes do vetor de regressão $\beta$, é recomendável substituir (3.9) pela classe dos M-Estimadores, dada por:

$$
\min _{\beta_{1}, \ldots, \beta_{q} \in \mathbb{R}} \sum_{k=1}^{n} \rho\left(\frac{\varepsilon_{k}}{\sigma}\right)
$$

em que $\sigma$ é um fator de escala e $\rho$ é uma função que goza das seguintes características: não negatividade, passagem pela origem, paridade e monotonia não decrescente em relação ao valor absoluto dos erros (Souza, 1998). Como $\sigma$ é desconhecido, ele costuma ser substituído por:

$$
\hat{\sigma}=1,4826 \cdot \operatorname{mediana}\left\{\left|\hat{\varepsilon}_{i_{1}}\right|, \ldots,\left|\hat{\varepsilon}_{i_{m}}\right|\right\},
$$

sendo $\left\{i_{1}, \ldots, i_{m}\right\} \subseteq\{1, \ldots, n\}(m, n \in \mathbb{N}, m \leq n)$ um subconjunto de $m$ índices associados a resíduos $\hat{\varepsilon}_{k}=y_{k}-\xi_{k}$ não nulos, com $k \in\{1, \ldots, n\}$ (Bulhões e Lima, 2010). 
Particularizações de $\rho$ conduzem a diversos estimadores. Por exemplo, $\rho\left(\varepsilon_{k}\right)=\varepsilon_{k}^{2} / 2$ coincide com o estimador de Mínimos Quadrados Ordinários, $\rho\left(\varepsilon_{k}\right)=\left|\varepsilon_{k}\right|$ é o estimador robusto da Norma $\mathrm{L}_{1}$ e $\rho\left(\varepsilon_{k}\right)=-\ln f\left(\varepsilon_{k}\right)$ é o estimador de Máxima Verossimilhança, com $f(\cdot)$ designando a função de verossimilhança. Notando esse fato, é claro que um M-Estimador só é robusto para certas $\rho$-funções.

Chamando os pontos $a, b$ e $c$ de nós, as $\rho$-funções mais utilizadas são:

- Função de Huber:

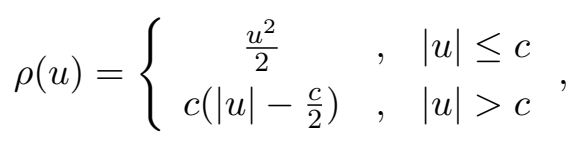

com

$$
\psi(u)=\rho^{\prime}(u)=\min \{c, \max \{u,-c\}\}=\left\{\begin{array}{cc}
-c, & u<-c \\
u, & |u| \leq c \\
c, & u>c
\end{array}\right.
$$

em que $u \in \mathbb{R}$ e a adoção da constante reguladora $c=1,345$ resulta em eficiência de $94,9 \%$ sob normalidade (Mendes, 1999).

- Função Redescendente de Hampel:

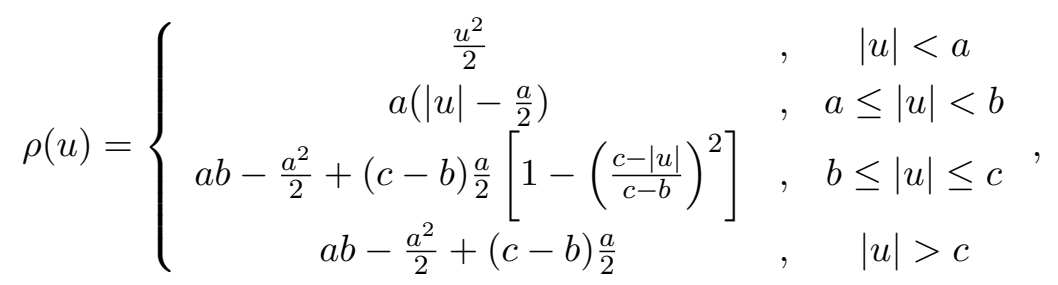

com

$$
\psi(u)=\rho^{\prime}(u)=\left\{\begin{array}{ccc}
u & , u \mid<a \\
a \cdot \operatorname{sgn}(u) & , & a \leq|u|<b \\
a \cdot \operatorname{sgn}(u) \cdot \frac{c-|u|}{c-b} & , & b \leq|u| \leq c \\
0 & , & |u|>c
\end{array},\right.
$$

em que $\operatorname{sgn}(\cdot)$ designa a função sinal e $u \in \mathbb{R}$.

- Função Biponderada de Tukey:

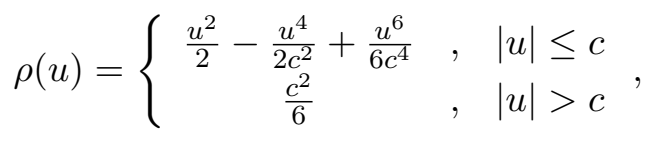

com

$$
\psi(u)=\rho^{\prime}(u)=\left\{\begin{array}{cc}
u\left(1-\frac{u^{2}}{c^{2}}\right)^{2} & , \quad|u| \leq c \\
0 & , \quad|u|>c
\end{array},\right.
$$

em que $u \in \mathbb{R}$ e a escolha de $c=4,685$ para a constante reguladora resulta em $94,9 \%$ de eficiência sob normalidade (Mendes, 1999).

Uma lista de $\rho$-funções e suas $\psi$-derivadas pode ser encontrada em Hoaglin et al. (1992).

Apesar da classe dos M-Estimadores ser flexível, o ponto de ruptura desses estimadores é $1 / n$, em virtude de eles não contemplarem os outliers provenientes do espaço das variáveis observadas exógenas. Para contornar esta limitação, Rousseeuw (1984) desenvolveu o estimador Menor 
Mediana dos Quadrados (MMQ), dado por:

$$
\hat{\beta}=\arg \min _{\beta \in \mathbb{R}^{q \times 1}} \operatorname{mediana}\left\{\varepsilon_{1}^{2}, \ldots, \varepsilon_{n}^{2}\right\} .
$$

Segundo Mendes (1999), o MMQ possui ponto de ruptura igual a 0,5, o que significa que ele é capaz de identificar o padrão sugerido por pelo menos metade dos dados. Como desvantagem, esse estimador é bastante ineficiente quando os erros são normalmente distribuídos, com taxa de convergência $\sqrt[3]{n}$.

A fim de combinar robustez e eficiência, Rousseeuw (1984) também desenvolveu o estimador Mínimos Quadrados Podados (MQP), dado por:

$$
\hat{\beta}=\arg \min _{\beta \in \mathbb{R}^{q \times 1}} \sum_{k=1}^{h} \varepsilon_{(k)}^{2},
$$

em que $\varepsilon_{(1)}^{2} \leq \cdots \leq \varepsilon_{(h)}^{2} \leq \cdots \leq \varepsilon_{(n)}^{2}$ são os quadrados dos erros ordenados, com $h \leq n, h, n \in \mathbb{N}$.

Escolhendo $h$ como o máximo inteiro contido no valor ótimo $\frac{n+q+1}{2}$, a taxa de convergência do MQP é $\sqrt{n}$, sendo superior à do MMQ (Mendes, 1999). Tomando $h \approx \frac{n}{2}$, a eficiência sob erros normalmente distribuídos do MQP é igual a 7\%, também considerada baixa (Conceição, 2004).

O S-Estimador é o vetor $\hat{\beta} \in \mathbb{R}^{q \times 1}$ dado por:

$$
\hat{\beta}=\arg \min _{\beta \in \mathbb{R}^{q \times 1}} Q(\beta),
$$

sendo a função de dispersão $Q$ a solução de

$$
\frac{1}{n-q} \sum_{k=1}^{n} \rho\left(\frac{\varepsilon_{k}}{Q}\right)=b,
$$

em que $\rho$ possui as mesmas características das funções redescendentes dos M-Estimadores (podendose escolher a Função Biponderada de Tukey, por exemplo), com $\lim _{u \rightarrow+\infty} \rho(u)=1$ e, geralmente, $0<b<1$. Tem-se também que $\hat{\beta}$ e $Q(\hat{\beta})$ são estimativas assintoticamente consistentes para $\beta$ e $Q(\beta)$, respectivamente. Seu ponto de ruptura é $\epsilon_{n}^{*}=\min \{b, 1-b\}$ (Maronna et al., 2006).

Uma característica de um S-Estimador é que o aumento de sua eficiência assintótica sob normalidade implica na diminuição de seu ponto de ruptura, pelo que ambos dependem de $b$. Ilustrando: se $\rho$ é a Função Biponderada de Tukey de parâmetro $c=2,9366$ e se $b=0,25$, então $\epsilon_{n}^{*}=\min \{0,25,1-0,25\}=0,25$ e a eficiência assintótica sob normalidade é de $75,9 \%$.

Pires e Branco (2007) sumarizam: MQO possui eficiência máxima e ponto de ruptura converge para zero; MMQ, MQP e S têm baixa eficiência e elevado ponto de ruptura; e M pode ser bastante eficiente para algumas $\rho$-funções, mas seu ponto de ruptura é nulo no limite.

Já foram desenvolvidos outros estimadores que combinam alto ponto de ruptura e mais eficiência estatística, podendo-se citar MM-Estimadores, uma classe de estimadores bastante eficiente sob normalidade e que possui ponto de ruptura aproximadamente igual a 50\% (Miranda, 2009). De acordo com Mendes (1999), um MM-Estimador é obtido em três estágios, a saber:

1. Obter estimativas de regressão com alto ponto de ruptura (via MMQ, por exemplo).

2. Calcular um M-Estimador de escala usando os resíduos obtidos no primeiro estágio. 
3. Fixando o estimador de escala do passo anterior como uma escala auxiliar, calcular um MEstimador de regressão baseado em uma $\rho$-função com $\psi$-derivada redescendente.

O método descrito chama-se MM-Estimador por usar dois M-Estimadores — um de regressão e outro de escala. Seu bom desempenho é justificado pela combinação de procedimentos que isoladamente fornecem alto ponto de ruptura ou (exclusive) alta eficiência sob normalidade. Por exemplo, certas combinações de estimadores conduzem a $\epsilon_{n}^{*}=0,25$ e, sob a suposição de erros gaussianos, eficiência em torno de $85 \%$. Como desvantagem, o tempo de processamento das MM-Estimativas é considerado elevado - uma consequência de seu complexo problema computacional.

O modelo de Análise Fatorial Exploratória com $s$ fatores ou constructos $\xi_{1}, \ldots, \xi_{s}$ é dado por

$$
\forall i \in\{1, \ldots, q\}, k \in\{1, \ldots, n\}, \quad x_{k, i}=\sum_{j=1}^{s} \lambda_{i, j}^{(\boldsymbol{x})} \xi_{k, j}+\delta_{k, i},
$$

em que $\xi_{k, j}$ é a $k$-ésima observação da $j$-ésima variável latente $\xi_{j}$ de $\boldsymbol{\xi}$ e $\delta_{k, i}$ é a $k$-ésima observação relacionada à variável de erro $\delta_{i}$ de $\boldsymbol{\delta}$.

A Figura 3.2 mostra a sugestão de Lattin et al. (2011) para representar graficamente o modelo estatístico descrito pela expressão (3.23), no caso particular de se ter duas variáveis latentes exógenas operacionalizadas por cinco variáveis observadas exógenas.

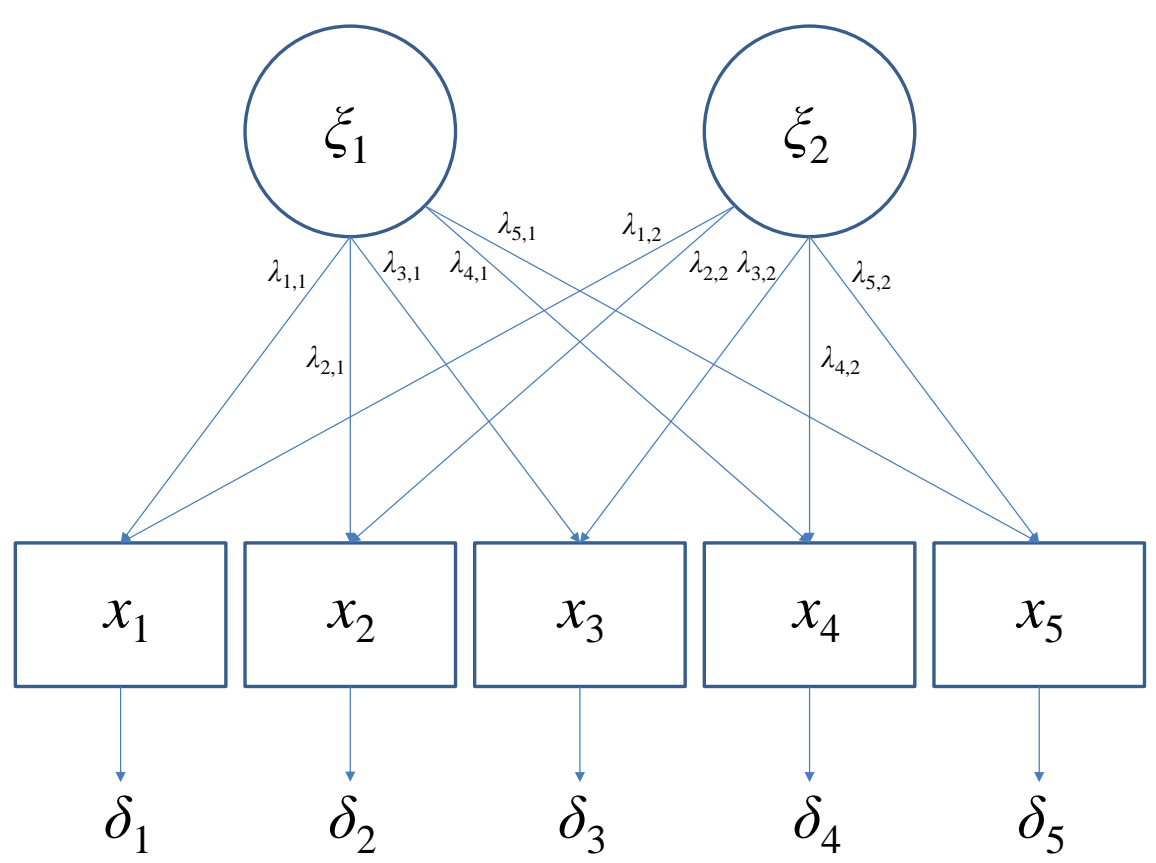

Figura 3.2: Representação gráfica de um Modelo de Análise Fatorial Exploratória.

Segundo Filzmoser (2002), estimativas robustas $\hat{\lambda}_{i, j}^{(\boldsymbol{x})}$ dos parâmetros do modelo (3.23) podem ser obtidas a partir de métodos robustos de estimação da Análise de Regressão. Por exemplo, para aplicar o M-Estimador, basta resolver o seguinte problema de otimização a cada $i \in\{1, \ldots, q\}$ fixo:

$$
\min _{\lambda_{i, 1}^{(\boldsymbol{x})}, \ldots, \lambda_{i, s}^{(\boldsymbol{x})} \in \mathbb{R}} \sum_{k=1}^{n} \rho\left(\delta_{k, i}\right)
$$


Rosado (2006) discute outliers no foro da Análise de Componentes Principais. Alguns métodos robustos de estimação aplicáveis neste caso podem ser conferidos em Filzmoser (2002).

\subsubsection{Abordagem confirmatória}

Os modelos de Análise de Equações Estruturais e Análise Fatorial Confirmatória utilizam a matriz de covariâncias clássicas ou ordinárias no processo de estimação de parâmetros através da minimização de alguma função de discrepância. Como as entradas dessa matriz são afetadas pela presença de outliers nos dados (Rousseeuw e Leroy, 2003), é razoável supor que os parâmetros estimados também estejam contaminados pela referida perturbação.

Assim como ocorre para os modelos da abordagem exploratória, o uso de métodos robustos de estimação é incentivado ao pesquisador que necessite aplicar modelos de análise confirmatória em dados que possuam valores atípicos. Por isso, as matrizes de covariâncias robustas são contempladas a fim de obter estimativas de MEEs resistentes a outliers (Yuan e Bentler, 1998), devendo-se substituir $F(\boldsymbol{S}, \boldsymbol{\Sigma}(\boldsymbol{\theta}))$ por $F(\boldsymbol{C}, \boldsymbol{\Sigma}(\boldsymbol{\theta}))$ no processo de obtenção de soluções, em que $\boldsymbol{C} \neq \boldsymbol{S}$ é uma matriz de covariâncias computada por um estimador robusto.

O estimador de Gnanadesikan-Kettenring (GK) visa estimar a covariância de duas variáveis aleatórias reais, digamos $U$ e $V$, pela relação

$$
\sigma_{(U, V)}=\frac{\sigma^{2}(U+V)-\sigma^{2}(U-V)}{4}
$$

em que $\sigma^{2}(\cdot)$ é uma representação funcional da variância que costumeiramente é obtida através de um método robusto (e.g., o quadrado do desvio-padrão aparado).

Havendo mais de duas variáveis aleatórias reais, utiliza-se a expressão (3.25) para estimar componente a componente e então formar a matriz de covariâncias $\hat{\boldsymbol{\Sigma}}_{\mathrm{GK}}$, a qual é simétrica, mas nem sempre positiva-semidefinida (Rocha, 2010).

Para ultrapassar essa limitação, o estimador de Gnanadesikan-Kettenring Ortogonalizado (GKO) foi desenvolvido. Ele consiste em aplicar uma transformação ${ }^{4}$ na matriz $\hat{\boldsymbol{\Sigma}}_{\text {GK }}$ para torná-la ortogonal e, assim, garantir que a resultante seja positiva-semidefinida sempre. Detalhes sobre este procedimento estão descritos na Seção B.3 do Apêndice B.

Uma outra classe de estimadores robustos para a matriz de covariâncias é inspirada nos métodos robustos para a Análise de Regressão. Ao invés de minimizar a soma de uma função dos resíduos para obter estimativas dos coeficientes lineares das variáveis observadas exógenas, o interesse se dá em minimizar distâncias $D_{k}\left(\boldsymbol{\omega}_{k}, \boldsymbol{\mu}, \boldsymbol{\Sigma}\right)$ para obter estimativas $\hat{\boldsymbol{\mu}} \in \mathbb{R}^{(p+q) \times 1}$ (vetor de locação) e $\hat{\boldsymbol{\Sigma}} \in \mathbb{R}^{(p+q) \times(p+q)}$ (matriz de covariâncias simétrica e semipositiva-definida, com det $\hat{\boldsymbol{\Sigma}}=1$ ) na perspectiva multivariada. Designando por $\boldsymbol{D}(\boldsymbol{Z}, \boldsymbol{\mu}, \boldsymbol{\Sigma})$ o vetor de componentes $D_{k}\left(\boldsymbol{\omega}_{k}, \boldsymbol{\mu}, \boldsymbol{\Sigma}\right)$ e um estimador robusto da dispersão por $\hat{\sigma}$, definem-se $\hat{\boldsymbol{\mu}}$ e $\hat{\boldsymbol{\Sigma}}$ como a solução de:

$$
\min _{\boldsymbol{\mu}, \boldsymbol{\Sigma}} \hat{\sigma}(\boldsymbol{D}(\boldsymbol{Z}, \boldsymbol{\mu}, \boldsymbol{\Sigma}))
$$

em que $\boldsymbol{Z}$ é uma matriz de dimensão $n \times(p+q)$, formada pelas $n$ observações multivariadas $\boldsymbol{\omega}_{k}$ das $p+q$ variáveis observadas, com $k \in\{1, \ldots, n\}$.

\footnotetext{
${ }^{4}$ Para um vetor aleatório real $\boldsymbol{u} \in \mathbb{R}^{m \times 1}$ e um vetor $\boldsymbol{a} \in \mathbb{R}^{m \times 1}$ qualquer, recorde-se que $\sigma^{2}\left(\boldsymbol{a}^{\prime} \boldsymbol{u}\right)=\boldsymbol{a}^{\prime} \sigma^{2}(\boldsymbol{u}) \boldsymbol{a}$, em que $\sigma$ é um funcional de escala, com $m \in \mathbb{N}$.
} 
O estimador Elipsoide de Volume Mínimo (EVM) para locação e escala é uma solução de (3.26) que considera $\hat{\sigma}$ a mediana amostral e se minimiza a mediana dos quadrados das distâncias, similar ao estimador de regressão MMQ (Rocha, 2010). Ele é definido como o elipsoide de menor volume entre todos os elipsoides $\{\boldsymbol{\omega}: D(\boldsymbol{\omega}, \boldsymbol{\mu}, \boldsymbol{\Sigma}) \leq 1\}$ que cobrem pelo menos $h$ pontos de $\boldsymbol{Z}$, sendo o valor ótimo de $h$ dado pelo maior inteiro menor que $n / 2+1$, cerca de $50 \%$ das observações.

Bastante análogo ao EVM, o estimador Covariância de Determinante Mínimo (CDM) para locação e escala também é uma solução de (3.26), porém considerando agora $\hat{\sigma}$ a escala aparada e a minimização da soma aparada dos quadrados das distâncias, semelhante ao estimador de regressão MQP (Rocha, 2010). Ele é definido como a média dos pelo menos $h$ pontos de $\boldsymbol{Z}$ associados aos elipsoides $\{\boldsymbol{\omega}: D(\boldsymbol{\omega}, \boldsymbol{\mu}, \boldsymbol{\Sigma}) \leq 1\}$ para os quais o determinante da matriz de covariâncias correspondente é mínimo, sendo o valor ótimo de $h$ dado pelo maior inteiro menor que $n-[n-(p+q)] / 2$.

Os estimadores EVM e CDM são importantes na análise de dados por possuírem ponto de ruptura aproximadamente igual a 0,5, mas já existem alternativas estatisticamente mais eficientes que eles, como as versões multivariadas dos estimadores S e MM.

Seja $T(\boldsymbol{Z}) \in \mathbb{R}^{(p+q) \times 1}$ um vetor e também seja $\boldsymbol{C}(\boldsymbol{Z})$ uma matriz de covariâncias simétrica positiva-definida. Os S-Estimadores para locação e escala multivariados são dados pela minimização $\operatorname{de} \operatorname{det} \boldsymbol{C}$, sujeita a

$$
\frac{1}{n} \sum_{k=1}^{n} \rho_{0}\left(\sqrt{\left(\boldsymbol{\omega}_{k}-T\right)^{\prime} \boldsymbol{C}\left(\boldsymbol{\omega}_{k}-T\right)}\right)=b,
$$

em que $\rho_{0}$ é uma função positiva, limitada, simétrica, estritamente crescente em $[0 ; c]$ e constante no intervalo $] c ;+\infty[$, enquanto $b$ assume valores em $] 0 ; \sup \rho_{0}$ [, conforme Mendes (1999). A mesma autora afirma que ele possui ponto de ruptura próximo a 50\%, com eficiência assintótica em torno de $33 \%$ sob normalidade, convergindo à taxa de $\sqrt{n}$.

Seja $\hat{\Sigma}_{\mathrm{S}}$ a matriz de covariâncias obtida pelo S-Estimador. As versões multivariadas dos MMEstimadores de locação e escala são dadas pela minimização de $\operatorname{det} \boldsymbol{G}$, sujeita a

$$
\frac{1}{n} \sum_{k=1}^{n} \rho_{1}\left(\sqrt{\left(\boldsymbol{\omega}_{k}-T\right)^{\prime} \boldsymbol{G}\left(\boldsymbol{\omega}_{k}-T\right)} / \hat{\sigma}_{n}\right)=b,
$$

em que $\rho_{1}$ goza das mesmas propriedades de $\rho_{0}$ e $\hat{\sigma}_{n}=\sqrt[2(p+q)]{\operatorname{det} \hat{\Sigma}_{\mathrm{S}}}$ (Salibian-Barrera et al., 2006).

Do mesmo modo que ocorre no contexto da Análise de Regressão, o MM-Estimador multivariado combina métodos robustos de estimação para obter alto ponto de ruptura e alta eficiência assintótica sob normalidade. Essas duas características desejáveis é que o tornam interessante.

Outra maneira de obter estimativas de parâmetros de MEEs resistentes à presença de outliers é caracterizada pela aplicação dos métodos robustos de regressão vistos na subseção anterior. Como essa abordagem está fora do escopo, recomenda-se a leitura de Cirillo e Barroso (2012b) ao leitor interessado em entender como os estimadores MMQ e MQP podem ser incorporados a MEEs. 


\section{Capítulo 4}

\section{Indicadores Clássicos e Robustos}

Uma maneira de julgar se um MEE está bem ajustado é a partir da análise do desempenho de alguns indicadores ou índices. Embora eles não possam, individualmente, refletir sobre a bondade do ajuste - por cada um possuir vantagens e desvantagens —, o estudo de várias dessas medidas em conjunto pode ser informativo (Bollen, 1989).

Grosso modo, os índices têm por objetivo comparar dois modelos distintos: o modelo definido pelo pesquisador e o modelo que não possui restrições sobre os momentos populacionais, dito saturado. Em geral, eles são construídos com base na função de discrepância ou na matriz de resíduos.

Cirillo e Barroso (2012a) comentam que os indicadores mais tradicionais, aqui chamados de clássicos, são bastante sensíveis ao tamanho amostral: amostras "grandes" influenciam o pesquisador a refutar seu modelo, ao passo que amostras "pequenas" sugerem que o modelo escolhido não seja rejeitado quando comparado a outros que forneceriam ajustes mais satisfatórios. Além disso, os mesmos autores também propuseram modificações do Índice da Qualidade do Ajuste (ou GFI, de Goodness-of-Fit Index) baseadas nas matrizes de covariâncias robustas EVM e CDM, com a finalidade de contornar o fato de o GFI usual muitas vezes não captar que as estimativas dos parâmetros obtidas estão contaminadas por outliers.

No presente capítulo, os principais indicadores clássicos serão revisados, as propostas de indicadores robustos recentemente desenvolvidas por Cirillo e Barroso (2012a) serão discutidas e novas sugestões de índices robustos serão apresentadas.

\subsection{Indicadores clássicos}

Sobre as funções de discrepância discutidas no Capítulo 2, sabe-se que $(n-1) F_{\mathrm{MV}},(n-1) F_{\mathrm{MQO}}$ e $(n-1) F_{\mathrm{MQP}}$ são assintoticamente convergentes à lei Qui-Quadrado, com $(1 / 2)(p+q)(p+q+1)-t$ graus de liberdade, em que $t$ é o número de parâmetros livres do MEE (Bollen, 1989). Com base nesse resultado, o teste do Qui-Quadrado avalia a hipótese $\mathcal{H}_{0}: \boldsymbol{\Sigma}-\boldsymbol{\Sigma}(\boldsymbol{\theta})=0$.

Entretanto, Bollen e Long (1993) orientam cautela para utilizar o teste estatístico citado, pelo que a convergência à lei Qui-Quadrado assume que as variáveis observadas possuam curtoses empíricas próximas da curtose da lei Normal Padrão e que a amostra seja suficientemente grande.

Levando em conta a sensibilidade apontada, algumas medidas de diagnóstico sobre o ajuste de MEEs, denominadas indicadores ou índices, foram construídas a partir de $F$ e de $\boldsymbol{S}-\boldsymbol{\Sigma}(\widehat{\boldsymbol{\theta}})$, como alternativas de análise. Elas têm o objetivo de comparar dois modelos distintos: o modelo definido pelo pesquisador e o modelo que considera que as variáveis observadas são mutuamente 
não correlacionadas e assume que somente são estimadas as variâncias das variáveis observadas, dito saturado.

A seguir, será feita uma revisão da literatura sobre os indicadores tradicionalmente mais utilizados em aplicações, aqui chamados de clássicos.

\subsubsection{Raiz Quadrada Média Padronizada}

Em Fan e Sivo (2005), pode ser conferida a seguinte expressão para o indicador Raiz Quadrada Média Padronizada (ou SRMR, de Standardized Root Mean Square Residual):

$$
\mathrm{SRMR}=\sqrt{\left\{2 \sum_{i=1}^{p+q} \sum_{j=1}^{i}\left[\left(s_{i j}-\sigma_{i j}(\hat{\boldsymbol{\theta}})\right) /\left(s_{i i} s_{j j}\right)\right]^{2}\right\} /[(p+q)(p+q+1)]}
$$

em que $s_{i j}$ e $\sigma_{i j}(\hat{\boldsymbol{\theta}})$ são as respectivas entradas genéricas de $\boldsymbol{S}$ e $\boldsymbol{\Sigma}(\hat{\boldsymbol{\theta}}), p$ é o número de variáveis observadas endógenas e $q$ é o número de variáveis observadas exógenas.

O índice apresentado assume que o modelo estimado é o correto e aponta que o modelo está bem ajustado à medida que se aproxima de zero, sendo desejável um valor em $[0 ; 0,08[$.

\subsubsection{Raiz do Erro Quadrático Médio de Aproximação}

O indicador Raiz do Erro Quadrático Médio de Aproximação (ou RMSEA, de Root Mean Square Error of Approximation) é dado por:

$$
\text { RMSEA }=\sqrt{\frac{\hat{F}_{0}}{\text { g.l.P }}}
$$

em que $\hat{F}_{0}=\max \left\{\left(\hat{C}-\right.\right.$ g.l. $\left.\left.{ }_{\mathrm{P}}\right) / n, 0\right\}$ e $\hat{C}=n \times F_{\mathrm{P}}$, sendo que $F_{\mathrm{P}}$ e g.l. $\mathrm{P}$ respectivamente são a função de discrepância e o número de graus de liberdade, ambos referentes ao modelo do pesquisador.

De acordo com Melhado (2004), o índice RMSEA considera a seguinte regra de decisão sobre o ajuste: está bom, se forem encontrados valores abaixo de 0,05; é razoável, quando entre 0,05 e 0,10 ; e pobre, caso seja superior a 0,10 . Ele é mais indicado para dados populacionais ou amostras grandes, em estratégias confirmatórias ou competitivas (Latif, 2000).

Uma estimativa intervalar de RMSEA com confiança $(1-\alpha) \times 100 \%$ é:

$$
\sqrt{\frac{\delta_{\mathrm{LI}}}{\mathrm{g} \cdot l_{\mathrm{P}}(n-1)}} ; \sqrt{\frac{\delta_{\mathrm{LS}}}{\mathrm{g} \cdot l_{\mathrm{P}}(n-1)}}[\text {, }
$$

em que $\delta_{\mathrm{LI}}$ e $\delta_{\mathrm{LS}}$ são tais que $f_{\chi_{\mathrm{NC}}^{2}}\left(\hat{C} \mid \delta_{\mathrm{LI}}\right.$, g.l. P $\left._{\mathrm{P}}\right)=1-\alpha / 2$ e $f_{\chi_{\mathrm{NC}}^{2}}\left(\hat{C} \mid \delta_{\mathrm{LS}}\right.$, g.l. $\left.\cdot \mathrm{P}\right)=\alpha / 2$, designando por $f_{\chi_{\mathrm{NC}}^{2}}(\hat{C} \mid \delta$, g.l.p $)$ a função de distribuição da lei Qui-Quadrado Não Central com parâmetro de não centralidade $\delta$ e g.l.p graus de liberdade, com $0<\alpha<1$. 


\subsection{3 Índice de Ajuste Normalizado}

O Índice de Ajuste Normalizado (ou NFI, de Normalized Fit Index) mede a redução na função de discrepância quando se passa do modelo saturado para o modelo do pesquisador. Ele é dado por:

$$
\mathrm{NFI}=\frac{F_{\mathrm{S}}-F_{\mathrm{P}}}{F_{\mathrm{S}}}
$$

em que $F_{\mathrm{S}}$ e $F_{\mathrm{P}}$ são os valores das funções de discrepância dos modelos saturado e do pesquisador, respectivamente.

Esse indicador assume valores em $[0 ; 1]$ e sugere que o modelo está bem ajustado à medida que se aproxima da unidade, tendo 0,9 como ponto de corte (Marôco, 2010).

Seu uso requer cuidados, pois o tamanho da amostra influencia a esperança matemática de NFI e ele também tende a aumentar com a adição de parâmetros, tal como ocorre com o coeficiente de determinação da Análise de Regressão (Melhado, 2004).

\subsection{4 Índice de Ajuste Corrigido}

Com o intuito de melhorar o NFI, o Índice de Ajuste Corrigido (ou IFI, de Incremental Fit Index) é uma proposta que visa diminuir a influência do tamanho amostral e da adição de parâmetros. Ele possui a seguinte expressão:

$$
\mathrm{IFI}=\frac{F_{\mathrm{S}}-F_{\mathrm{P}}}{F_{\mathrm{S}}-\left[\mathrm{g} \cdot \cdot_{\mathrm{P}} /(n-1)\right]}
$$

em que g.l.p é o número de graus de liberdade do modelo do pesquisador, já $F_{\mathrm{S}}$ e $F_{\mathrm{P}}$ são os respectivos valores das funções de discrepância dos modelos saturado e do pesquisador.

Esse indicador sugere que valores próximos da unidade representam modelos válidos, mas ele não varia necessariamente em $[0 ; 1]$, sugerindo problemas de superajuste quando são encontrados valores muito grandes (Melhado, 2004). Além disso, as diferenças entre NFI e IFI diminuem à medida que o tamanho da amostra aumenta.

\subsection{5 Índice de Tucker-Lewis ou Îndice de Ajuste Não Normalizado}

O Índice de Tucker-Lewis (ou TLI, de Tucker-Lewis Index), também conhecido por Índice de Ajuste Não Normalizado (ou NNFI, de Non-Normed Fit Index), visa mensurar a redução da função de discrepância de quando se passa do modelo saturado para o modelo do pesquisador, considerando os graus de liberdade. Ele é calculado como:

$$
\mathrm{TLI}=\mathrm{NNFI}=\frac{\left(F_{\mathrm{S}} / \mathrm{g}_{\mathrm{l}} \mathrm{s}_{\mathrm{S}}\right)-\left(F_{\mathrm{P}} / \mathrm{g} \cdot \mathrm{l}_{\mathrm{P}}\right)}{\left(F_{\mathrm{S}} / \mathrm{g} \cdot \mathrm{l}_{\mathrm{S}}\right)-[1 /(n-1)]}
$$

em que $F_{\mathrm{S}}$ e $F_{\mathrm{P}}$ são as respectivas funções de discrepância dos modelos saturado e do pesquisador, enquanto que g.l.s e g.l.p são os correspondentes graus de liberdade dos modelos mencionados.

Conforme Melhado (2004), valores em $[0,9 ; 1]$ indicam bom ajuste sem depender do tamanho da amostra, mas o índice não está restrito em $[0 ; 1]$. A mesma autora também pontua que valores negativos sugerem problemas de má especificação e que valores acima da unidade levantam suspeita de superajustamento. 


\subsection{6 Índice de Ajuste Comparativo}

Também assumindo valores em [0;1], Widaman e Thompson (2003) apresentam a seguinte expressão para o Índice de Ajuste Comparativo (ou CFI, de Comparative Fit Index):

$$
\mathrm{CFI}=1-\frac{\max \left(\left\{F_{\mathrm{P}}-\left[\mathrm{g} \cdot \mathrm{l}_{\mathrm{P}} /(n-1)\right]\right\}, 0\right)}{\max \left(\left\{F_{\mathrm{P}}-\left[\mathrm{g} \cdot \mathrm{l}_{\mathrm{P}} /(n-1)\right]\right\},\left\{F_{\mathrm{S}}-\left[\mathrm{g} \cdot \mathrm{l}_{\mathrm{S}} /(n-1)\right]\right\}, 0\right)},
$$

em que $F_{\mathrm{S}}$ e $F_{\mathrm{P}}$ são as respectivas funções de discrepância dos modelos saturado e do pesquisador, ao passo que g.l. S $_{\text {e g.l. }}$ P são os correspondentes graus de liberdade dos modelos mencionados.

Sem ser influenciado pelo tamanho da amostra, porém sendo afetado pela adição de variáveis, o indicador CFI qualifica o modelo como: ruim, se estiver em [0;0,90]; bom, quando entre $] 0,90 ; 0,95]$; ou muito bom, caso pertença a ]0,95; 1].

\subsection{7 Índice Relativo de Não Centralidade}

Widaman e Thompson (2003) descrevem o Índice Relativo de Não Centralidade (ou RNI, de Relative Noncentrality Index) a partir da seguinte expressão:

$$
\mathrm{RNI}=1-\frac{F_{\mathrm{P}}-\left[\mathrm{g} \cdot \mathrm{l}_{\mathrm{P}} /(n-1)\right]}{F_{\mathrm{S}}-\left[\mathrm{g} \cdot \mathrm{l}_{\mathrm{S}} /(n-1)\right]}
$$

em que $F_{\mathrm{S}}$ e $F_{\mathrm{P}}$ são as respectivas funções de discrepância dos modelos saturado e do pesquisador, enquanto que g.l. .S $_{\text {g.l.P }}$ são os correspondentes graus de liberdade dos modelos mencionados.

Os mesmos autores justificam que valores acima de 0,95 são aceitáveis.

Os dois últimos indicadores a serem apresentados nesta subseção merecem uma atenção especial aos objetivos do presente trabalho, por serem desenvolvidos diretamente a partir da matriz de covariâncias amostrais.

\subsection{8 Índices da Qualidade do Ajuste}

O Índice da Qualidade do Ajuste (ou GFI, de Goodness-of-Fit Index) apresenta distintas formas, variando conforme o método de estimação. A seguir, as versões do GFI para MV, MQO e MQG:

$$
\begin{gathered}
\mathrm{GFI}_{\mathrm{MV}}=1-\frac{\operatorname{tr}\left[\left(\boldsymbol{\Sigma}^{-1}(\widehat{\boldsymbol{\theta}}) \boldsymbol{S}-\boldsymbol{I}\right)^{2}\right]}{\operatorname{tr}\left[\left(\boldsymbol{\Sigma}^{-1}(\widehat{\boldsymbol{\theta}}) \boldsymbol{S}\right)^{2}\right]}, \\
\mathrm{GFI}_{\mathrm{MQO}}=1-\frac{\operatorname{tr}\left[(\boldsymbol{S}-\boldsymbol{\Sigma}(\hat{\boldsymbol{\theta}}))^{2}\right]}{\operatorname{tr}\left[\boldsymbol{S}^{2}\right]}
\end{gathered}
$$

$\mathrm{e}$

$$
\mathrm{GFI}_{\mathrm{MQG}}=1-\frac{\operatorname{tr}\left[\left(\boldsymbol{I}-\boldsymbol{\Sigma}(\hat{\boldsymbol{\theta}}) \boldsymbol{S}^{-1}\right)^{2}\right]}{p+q},
$$

em que $\hat{\boldsymbol{\theta}}$ é um vetor de estimativas do vetor de parâmetros $\boldsymbol{\theta}, \boldsymbol{\Sigma}(\hat{\boldsymbol{\theta}})$ é a matriz de covariâncias $\boldsymbol{\Sigma}(\boldsymbol{\theta})$ avaliada no vetor $\hat{\boldsymbol{\theta}}$ que minimiza a função de discrepância $F$ considerada, $\boldsymbol{S}$ é a matriz de covariâncias de $\boldsymbol{z}=\left[\begin{array}{ll}\boldsymbol{y}^{\prime} & \boldsymbol{x}^{\prime}\end{array}\right]^{\prime}, \operatorname{tr}(\cdot)$ designa a função traço, $\boldsymbol{I}$ é a matriz identidade, $p$ é o número 
de componentes de $\boldsymbol{y}$ e $q$ é o número de componentes de $\boldsymbol{x}$.

Independentemente da função de discrepância, os indicadores GFI buscam medir a quantidade relativa de covariâncias $\boldsymbol{S}$ que são preditas por $\boldsymbol{\Sigma}(\hat{\boldsymbol{\theta}})$. Eles podem eventualmente assumir valores negativos e possuem a seguinte característica: GFI $=1$ se, e só se, $\boldsymbol{S}=\boldsymbol{\Sigma}(\hat{\boldsymbol{\theta}})$. Em palavras, o ajuste do MEE é perfeito somente quando se obtém um GFI unitário (Tanaka e Huba, 1985).

A partir de revisões da literatura e experiências práticas, Marôco (2010) sugere aos valores de GFI a seguinte interpretação sobre a bondade do ajustamento: se GFI $<0,80$, está mau; se $0,80 \leq$ GFI $<0,90$, está sofrível; se $0,90 \leq$ GFI $<0,95$, está bom; e se GFI $\geq 0,95$, está muito bom.

\subsection{9 Índices Corrigidos da Qualidade do Ajuste}

Com o intuito de refinar o GFI aos graus de liberdade e à quantidade de variáveis observadas do MEE, o Índice Corrigido da Qualidade do Ajuste (ou AGFI, de Adjusted Goodness-of-Fit Index) foi proposto e também se modifica de acordo ao método de estimação escolhido, possuindo as seguintes versões para MV, MQO e MQG:

$$
\begin{aligned}
\mathrm{AGFI}_{\mathrm{MV}} & =1-\left[\frac{(p+q)(p+q+1)}{2 \mathrm{~g} \cdot \mathrm{l}_{\mathrm{P}}}\right]\left(1-\mathrm{GFI}_{\mathrm{MV}}\right), \\
\mathrm{AGFI}_{\mathrm{MQO}} & =1-\left[\frac{(p+q)(p+q+1)}{2 \mathrm{~g} \cdot \mathrm{l}_{\mathrm{P}}}\right]\left(1-\mathrm{GFI}_{\mathrm{MQO}}\right)
\end{aligned}
$$

$\mathrm{e}$

$$
\mathrm{AGFI}_{\mathrm{MQG}}=1-\left[\frac{(p+q)(p+q+1)}{2 \mathrm{~g} \cdot \mathrm{l}_{\mathrm{P}}}\right]\left(1-\mathrm{GFI}_{\mathrm{MQG}}\right),
$$

em que g.l.p é o número de graus de liberdade do modelo do pesquisador, $p$ é o número de variáveis endógenas, $q$ é o número de variáveis exógenas e, por fim, $\mathrm{GFI}_{\mathrm{MV}}, \mathrm{GFI}_{\mathrm{MQO}}$ e $\mathrm{GFI}_{\mathrm{MQG}}$ sequencialmente são os indicadores GFI ajustados por MV, MQO e MQG.

O indicador AGFI também pode assumir valores negativos, assim como o GFI. Além disso, verifica-se facilmente que GFI $=1$ se, e somente se, AGFI $=1$. Ambos possuem a mesma interpretação e compartilham o defeito de serem inflacionados à medida que o número de unidades amostrais aumenta.

Índices similares aos aqui revisados e outras medidas voltadas à comparação de modelos podem ser vistas em Melhado (2004). Mais desenvolvimentos podem ser conferidos em Bertossi (2012).

\subsection{Indicadores robustos - propostas alternativas}

Conforme já mencionado no Capítulo 2, Marôco (2010) comenta que um dos pressupostos da modelagem de equações estruturais é que os dados não estejam contaminados por outliers. Na mesma obra, este autor faz duas recomendações de medidas para a detecção de tais pontos:

- Análise univariada: usando as regras empíricas de um boxplot, deve-se calcular as barreiras inferior e superior para detectar valores atípicos em cada uma das $p+q$ variáveis observadas.

- Análise multivariada: sob a assunção de normalidade multivariada ou amostras "grandes", a estatística a seguir, proveniente da curtose multivariada, pode ser utilizada para diagnosticar 
outliers multivariados:

$$
Z=\frac{\frac{1}{n} \sum_{k=1}^{n} D_{k}^{4}(\boldsymbol{S})-\{[(p+q)(p+q+2)(n-1)] /(n+1)\}}{\sqrt{[8(p+q)(p+q+2)] / n}},
$$

cuja distribuição assintótica é a lei Normal Padrão, em que

$$
\forall k \in\{1, \ldots, n\}, \quad D_{k}(\boldsymbol{S})=\sqrt{\left(\boldsymbol{\omega}_{k}-\overline{\boldsymbol{z}}\right)^{\prime} \boldsymbol{S}^{-1}\left(\boldsymbol{\omega}_{k}-\overline{\boldsymbol{z}}\right)}
$$

é a distância de Mahalanobis da $k$-ésima observação multivariada $\boldsymbol{\omega}_{k}$ das $p+q$ variáveis observadas, $\overline{\boldsymbol{z}}$ é o centroide das variáveis observadas (vetor de médias) e $\boldsymbol{S}$ é a matriz de covariâncias clássicas ou ordinárias definida em (2.10).

Contudo, esses métodos mais tradicionais nem sempre fornecem resultados fidedignos quanto à deteç̧ão de outliers.

Paula (2013) aponta que gráficos de diagnóstico com quantidades excessivas de outliers costumam sugerir que a distribuição amostral não se adequa à lei de probabilidade assumida nos pressupostos do modelo. Investigando os boxplots, Hubert e Vandervieren (2008) criticam a regra empírica usualmente considerada nos cálculos, argumentando a necessidade de atribuir pesos diferentes para as barreiras inferior e superior quando a distribuição amostral é oblíqua. No caso de assimetria positiva, uma discussão sobre o que são outliers em populações Gama e Exponencial pode ser conferida Rosado (2006).

Do ponto de vista multivariado, Mendes (1999) utiliza dados reais para mostrar que o elipsoide de tolerância de nível $\alpha \cdot 100 \%$

$$
D_{k}(\boldsymbol{C}) \leq \sqrt{\chi_{\alpha, p+q-1}^{2}}, \quad 0<\alpha<1
$$

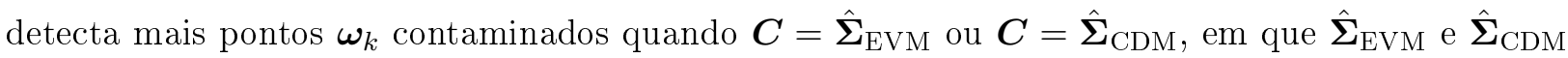
respectivamente são as matrizes de covariâncias estimadas pelos métodos robustos EVM e CDM vistos no Capítulo 3 e $\chi_{\alpha, p+q+1}^{2}$ é o $\alpha$-quantil da lei Qui-Quadrado com $p+q-1$ graus de liberdade.

O gráfico DD é muito útil para visualizar outliers multivariados. Ele é formado pela nuvem bivariada de $n$ pontos resultantes do cruzamento de distâncias clássicas (abscissas) e robustas (ordenadas). Dividindo o plano cartesiano em quatro partes delimitadas por duas retas iguais ao valor $\sqrt{\chi_{\alpha, p+q+1}^{2}}$ na horizontal e na vertical, tem-se a seguinte interpretação: "bons" pontos estão próximos à primeira bissetriz e localizados na parte inferior esquerda; estando nas demais regiões, são "maus" pontos. Sua formulação para MEEs está desenvolvida em Yuan e Hayashi (2010).

Outras técnicas de identificação e investigação de pontos influentes em MEEs com variáveis contínuas podem ser aprendidas em Tanaka e Odaka (1989) e também em Tanaka et al. (1991).

No Capítulo 3, a sensibilidade das estimativas dos parâmetros de MEEs computados a partir de amostras contaminadas foi abordada e o uso de métodos robustos foi recomendado. Já no início deste tópico, a utilização de técnicas sofisticadas de detecção de outliers antes do ajuste do modelo foi incentivada, devido à fragilidade mencionada. Para fechar a discussão, algumas formas de identificar se um MEE ajustado foi impactado por pontos atípicos serão contempladas.

Como o estudo dos indicadores clássicos caracteriza um dos poucos meios de julgar a bondade do ajuste de um MEE, torna-se necessário avaliar se isso é suficiente para identificar problemas 
relacionados a dados contaminados por outliers, ou se técnicas novas precisam ser introduzidas, motivando a realização do presente trabalho.

Brunelli (2012) analisou graficamente o comportamento de alguns indicadores clássicos sobre perturbações no tamanho da amostra, na assimetria, na curtose, na direção das flechas de influência direta entre duas variáveis e na qualidade dos dados, considerando multicolinearidade e outliers. A mesma autora apontou a necessidade de estudos adicionais nos casos em que ocorre a inclusão de valores atípicos em mais de uma variável.

Além disso, considerando que alguns métodos de estimação fornecem estimativas de parâmetros e estatísticas de interesse pouco confiáveis quando ocorre contaminação e desvios da normalidade multivariada, é razoável desconfiar que os resultados dos indicadores clássicos também estejam incorretos (Kirby e Bollen, 2009).

Focando nessa finalidade, Cirillo e Barroso (2012a) mostraram que o indicador GFI $_{\mathrm{MQO}}$ não é razoável para mostrar que um MEE está mal ajustado em decorrência da presença de outliers no conjunto de dados. Por se tratar de um índice que utiliza diretamente a matriz de covariâncias $\boldsymbol{S}$ em seu cálculo, eles a substituíram pelas matrizes de covariâncias robustas $\hat{\boldsymbol{\Sigma}}_{\mathrm{EVM}}$ e $\hat{\boldsymbol{\Sigma}}_{\mathrm{CDM}}$, assim propondo dois indicadores:

$$
\mathrm{GFI}_{\mathrm{MQO}}^{\mathrm{EVM}}=1-\frac{\operatorname{tr}\left[\left(\hat{\boldsymbol{\Sigma}}_{\mathrm{EVM}}-\boldsymbol{\Sigma}^{-1}(\widehat{\boldsymbol{\theta}})\right)^{2}\right]}{\operatorname{tr}\left(\hat{\boldsymbol{\Sigma}}_{\mathrm{EVM}}^{2}\right)}
$$

e

$$
\mathrm{GFI}_{\mathrm{MQO}}^{\mathrm{CDM}}=1-\frac{\operatorname{tr}\left[\left(\hat{\boldsymbol{\Sigma}}_{\mathrm{CDM}}-\boldsymbol{\Sigma}^{-1}(\widehat{\boldsymbol{\theta}})\right)^{2}\right]}{\operatorname{tr}\left(\hat{\boldsymbol{\Sigma}}_{\mathrm{CDM}}^{2}\right)},
$$

em que $\hat{\boldsymbol{\Sigma}}_{\mathrm{EVM}}$ e $\hat{\boldsymbol{\Sigma}}_{\mathrm{CDM}}$ respectivamente designam as matrizes de covariâncias ajustadas pelos métodos de estimação EVM e CDM, $\boldsymbol{\Sigma}^{-1}(\widehat{\boldsymbol{\theta}})$ é a inversa da matriz de covariâncias $\boldsymbol{\Sigma}(\boldsymbol{\theta})$ avaliada no vetor de estimativas de Mínimos Quadrados Ordinários e $\operatorname{tr}(\cdot)$ é a função traço.

De fato, o desempenho de $\mathrm{GFI}_{\mathrm{MQO}}^{\mathrm{EVM}}$ e $\mathrm{GFI}_{\mathrm{MQO}}^{\mathrm{CDM}}$ é bastante superior a do usual GFI $\mathrm{MQO}_{\mathrm{MQO}}$ quanto à capacidade de alertar o pesquisador que as estimativas dos parâmetros estão contaminadas por outliers (Cirillo e Barroso, 2012a). Assim, ambos são considerados indicadores robustos. Todavia, estudos adicionais sobre os casos de se ter elevadas quantidades de outliers provenientes de alguma distribuição com excesso de curtose ainda são necessários - uma das motivações para a realização do presente trabalho.

Com o objetivo de explorar mais esses novos desenvolvimento tomando o método de Máxima Verossimilhança como procedimento de estimação, esta dissertação considerou os indicadores

$$
\mathrm{GFI}_{\mathrm{MV}}^{\mathrm{EVM}}=1-\frac{\operatorname{tr}\left[\left(\boldsymbol{\Sigma}^{-1}(\widehat{\boldsymbol{\theta}}) \hat{\boldsymbol{\Sigma}}_{\mathrm{EVM}}-\boldsymbol{I}\right)^{2}\right]}{\operatorname{tr}\left[\left(\boldsymbol{\Sigma}^{-1}(\widehat{\boldsymbol{\theta}}) \hat{\boldsymbol{\Sigma}}_{\mathrm{EVM}}\right)^{2}\right]}
$$


$\mathrm{e}$

$$
\mathrm{GFI}_{\mathrm{MV}}^{\mathrm{CDM}}=1-\frac{\operatorname{tr}\left[\left(\boldsymbol{\Sigma}^{-1}(\widehat{\boldsymbol{\theta}}) \hat{\boldsymbol{\Sigma}}_{\mathrm{CDM}}-\boldsymbol{I}\right)^{2}\right]}{\operatorname{tr}\left[\left(\boldsymbol{\Sigma}^{-1}(\widehat{\boldsymbol{\theta}}) \hat{\boldsymbol{\Sigma}}_{\mathrm{CDM}}\right)^{2}\right]},
$$

como também novas propostas de modificação do $\mathrm{GFI}_{\mathrm{MV}}$ que utilizem outras matrizes de covariâncias robustas, pelo que os métodos de estimação EVM e CDM não são muito eficientes, apesar do alto ponto de ruptura. São elas:

$$
\begin{gathered}
\mathrm{GFI}_{\mathrm{MV}}^{\mathrm{GKO}}=1-\frac{\operatorname{tr}\left[\left(\boldsymbol{\Sigma}^{-1}(\widehat{\boldsymbol{\theta}}) \hat{\boldsymbol{\Sigma}}_{\mathrm{GKO}}-\boldsymbol{I}\right)^{2}\right]}{\operatorname{tr}\left[\left(\boldsymbol{\Sigma}^{-1}(\widehat{\boldsymbol{\theta}}) \hat{\boldsymbol{\Sigma}}_{\mathrm{GKO}}\right)^{2}\right]}, \\
\mathrm{GFI}_{\mathrm{MV}}^{\mathrm{S}}=1-\frac{\operatorname{tr}\left[\left(\boldsymbol{\Sigma}^{-1}(\widehat{\boldsymbol{\theta}}) \hat{\boldsymbol{\Sigma}}_{\mathrm{S}}-\boldsymbol{I}\right)^{2}\right]}{\operatorname{tr}\left[\left(\boldsymbol{\Sigma}^{-1}(\widehat{\boldsymbol{\theta}}) \hat{\boldsymbol{\Sigma}}_{\mathrm{S}}\right)^{2}\right]}
\end{gathered}
$$

$\mathrm{e}$

$$
\mathrm{GFI}_{\mathrm{MV}}^{\mathrm{MM}}=1-\frac{\operatorname{tr}\left[\left(\boldsymbol{\Sigma}^{-1}(\widehat{\boldsymbol{\theta}}) \hat{\boldsymbol{\Sigma}}_{\mathrm{MM}}-\boldsymbol{I}\right)^{2}\right]}{\operatorname{tr}\left[\left(\boldsymbol{\Sigma}^{-1}(\widehat{\boldsymbol{\theta}}) \hat{\boldsymbol{\Sigma}}_{\mathrm{MM}}\right)^{2}\right]},
$$

além das correspondentes versões robustas de $\mathrm{AGFI}_{\mathrm{MV}}$ :

$$
\begin{aligned}
\mathrm{AGFI}_{\mathrm{MV}}^{\mathrm{EVM}} & =1-\left[\frac{(p+q)(p+q+1)}{2 \mathrm{~g} \cdot \mathrm{l}_{\mathrm{P}}}\right]\left(1-\mathrm{GFI}_{\mathrm{MV}}^{\mathrm{EVM}}\right), \\
\mathrm{AGFI}_{\mathrm{MV}}^{\mathrm{CDM}} & =1-\left[\frac{(p+q)(p+q+1)}{2 \mathrm{~g} \cdot \mathrm{l} \cdot \mathrm{P}}\right]\left(1-\mathrm{GFI}_{\mathrm{MV}}^{\mathrm{CDM}}\right), \\
\mathrm{AGFI}_{\mathrm{MV}}^{\mathrm{GKO}} & =1-\left[\frac{(p+q)(p+q+1)}{2 \mathrm{~g} \cdot l_{\cdot} \mathrm{P}}\right]\left(1-\mathrm{GFI}_{\mathrm{MV}}^{\mathrm{GKO}}\right), \\
\mathrm{AGFI}_{\mathrm{MV}}^{\mathrm{S}} & =1-\left[\frac{(p+q)(p+q+1)}{2 \mathrm{~g} \cdot 1 \cdot \mathrm{P}}\right]\left(1-\mathrm{GFI}_{\mathrm{MV}}^{\mathrm{S}}\right)
\end{aligned}
$$

e

$$
\mathrm{AGFI}_{\mathrm{MV}}^{\mathrm{MM}}=1-\left[\frac{(p+q)(p+q+1)}{2 \mathrm{~g} \cdot \mathrm{l}_{\mathrm{P}}}\right]\left(1-\mathrm{GFI}_{\mathrm{MV}}^{\mathrm{MM}}\right),
$$

em que $\hat{\boldsymbol{\Sigma}}_{\mathrm{GKO}}, \hat{\boldsymbol{\Sigma}}_{\mathrm{EVM}}, \hat{\boldsymbol{\Sigma}}_{\mathrm{CDM}}, \hat{\boldsymbol{\Sigma}}_{\mathrm{S}}$ e $\hat{\boldsymbol{\Sigma}}_{\mathrm{MM}}$ respectivamente são as matrizes de covariâncias estimadas pelos métodos robustos GKO, EVM, CDM, S e MM, I é a matriz identidade de ordem $p+q$, $\boldsymbol{\Sigma}^{-1}(\widehat{\boldsymbol{\theta}})$ é a inversa da matriz de covariâncias $\boldsymbol{\Sigma}(\boldsymbol{\theta})$ avaliada no vetor de estimativas de Máxima Verossimilhança e $\operatorname{tr}(\cdot)$ é a função traço.

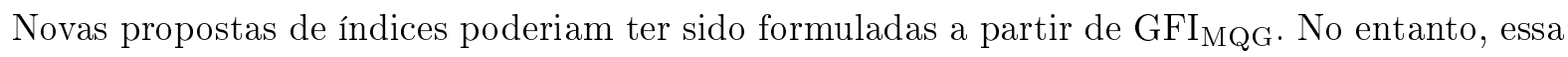
ideia foi descartada devido à similaridade entre os resultados de indicadores clássicos de MEEs computados pelos métodos de estimação MV e MQG em outro estudo (Brunelli, 2012). 


\section{Capítulo 5}

\section{Simulações}

Neste capítulo, as medidas SRMR, RMSEA, NFI, IFI, NNFI (ou TLI), CFI, RNI, GFI $\mathrm{GFI}_{\mathrm{MV}}^{\mathrm{GKO}}, \mathrm{GFI}_{\mathrm{MV}}^{\mathrm{EVM}}, \mathrm{GFI}_{\mathrm{MV}}^{\mathrm{CDM}}, \mathrm{GFI}_{\mathrm{MV}}^{\mathrm{S}}, \mathrm{GFI}_{\mathrm{MV}}^{\mathrm{MM}}, \mathrm{AGFI}_{\mathrm{MV}}, \mathrm{AGFI}_{\mathrm{MV}}^{\mathrm{GKO}}, \mathrm{AGFI}_{\mathrm{MV}}^{\mathrm{EV}}, \mathrm{AGFI}_{\mathrm{MV}}^{\mathrm{CDM}}, \mathrm{AGFI}_{\mathrm{MV}}^{\mathrm{S}}$ e $\mathrm{AGFI}_{\mathrm{MV}}^{\mathrm{MM}}$ serão avaliadas quanto à capacidade de diagnosticar se um modelo ajustado está, ou não, com suas estimativas distorcidas por conta da presença de outliers no conjunto de dados, considerando desvios de simetria, excesso de curtose e diferentes frações de contaminação, tamanhos de amostra, números de parâmetros e quantidades de variáveis perturbadas. Para tanto, estudos de simulação serão conduzidos em dois MEEs similares que possuem apenas o submodelo de mensuração em $\boldsymbol{x}$ (situação mais comum na prática), chamados também de modelo de Análise Fatorial Confirmatória, distinguindo-se entre si na quantidade de parâmetros a estimar.

Os tamanhos de amostra escolhidos, o procedimento adotado para afetar as variáveis com valores atípicos e outros aspectos mais gerais que foram necessários considerar nos estudos de simulação também serão detalhados nos próximos tópicos deste capítulo.

\subsection{Tamanhos de amostra}

Não existe um consenso quanto ao tamanho de amostra ideal para a aplicação de técnicas de Análise Multivariada. Em Análise de Regressão, a recomendação mais comum é a de que cada variável observada exógena contenha pelo menos cinco observações (Lattin et al., 2011).

Cada variável observada em MEE costuma corresponder a dois ou três parâmetros, variando conforme a quantidade de relações diretas e recíprocas estabelecidas, de coeficientes de correlação não nulos admitidos e de variâncias das variáveis de erro não fixadas. Assim, é razoável admitir que haja pelo menos cinco observações para cada parâmetro a ser estimado, neste caso.

Por outro lado, alguns estudos apontam que tamanhos elevados de amostra podem comprometer os resultados sugeridos pelos indicadores. Hox e Bechger (1998) perceberam que alguns índices construídos a partir da estatística do teste Qui-Quadrado são bastante sensíveis em relação à quantidade de observações. Hoyle e Panter (1995) e Shah e Goldstein (2006) mostram que conjuntos de dados que apresentam normalidade multivariada em distribuição para $n \geq 650$ não provocam efeitos em alguns indicadores padronizados e em índices comparativos.

Conciliando para que as amostras não sejam nem muito "pequenas" e também nem muito "grandes", aqui foram adotados os quatro tamanhos $n \in\{100,200,300,500\}$, os quais estão de acordo com várias sugestões da literatura sobre MEEs, conforme comentam Schumacker e Lomax (2004).

Ainda que nem sempre seja possível coletar muitos dados na prática, obrigando o pesquisador a 
eventualmente não seguir as recomendações sobre o número desejável de unidades, o caso $n=500$ é importante para simulações. Deve-se tomar amostras maiores que a de tamanho mínimo necessário para averiguar o impacto da perturbação inserida, o que elimina a suspeita que os desvios observados são decorrentes do uso de uma amostra que não era "grande" o bastante (Hair et al., 2010).

\subsection{Contaminações por outliers}

A geração das amostras contaminadas por outliers se dá pelo que está descrito na equação (3.2).

As variáveis observadas simuladas foram atingidas a partir de contaminações provocadas diretamente nas suas correspondentes variáveis de erro, com estas últimas tendo sido geradas através da lei Normal Padrão. A fim de ajustar MEEs considerando valores atípicos provenientes de desvios de simetria ou excesso de curtose na amostra, procedeu-se do seguinte modo: as distribuições empíricas tiveram parte de seus valores substituídos por valores oriundos de leis assimétricas positivas com suporte em $] 0 ;+\infty[$ ou por valores fornecidos a partir de leis simétricas em relação à média zero com "caudas pesadas" (i.e., platicúrticas), respectivamente.

Embora alguns métodos robustos possuam ponto de ruptura em torno de 0,50 ou 50\%, elevadas frações de contaminação podem ser questionadas, no sentido de elas serem mesmo outliers ou se há a necessidade de realizar análises para cada subpopulação identificada. Por isso, $\alpha=0,25$ foi a maior proporção de pontos atípicos apreciada.

Com o propósito de contemplar baixa e alta proporções de outliers, foram adotadas para o presente texto as duas seguintes frações de contaminação: $\alpha \in\{0,05,0,25\}$.

\subsection{Estudos de simulação}

As estratégias aqui adotadas para realizar os estudos de simulação basearam-se em alguns aspectos das metodologias usadas em Brunelli (2012) e Bistaffa (2011).

Os índices apresentados no Capítulo 4 foram analisados quanto à capacidade de detectar se um modelo cujas estimativas estão afetadas por outliers está insatisfatório. Para tanto, dois estudos de simulação serão conduzidos em MEEs que têm apenas o submodelo de mensuração em $\boldsymbol{x}$ (i.e., Análise Fatorial Confirmatória), diferindo-se entre si na quantidade de parâmetros a estimar.

Os parâmetros e indicadores dos dois modelos estimados foram computados mil vezes, considerando sempre os mesmos outliers em cada uma das mil replicações, quando fixados: o tamanho de amostra, a fração de contaminação e o tipo de perturbação provocado nos dados.

Com os mil valores de cada caso armazenados, as seguintes estatísticas foram calculadas: a média aritmética $\left(\hat{\mu}_{\hat{\theta}}\right)$ e o desvio-padrão $\left(\hat{\sigma}_{\hat{\theta}}\right)$ das estimativas de cada parâmetro; e a partir das avaliações de cada indicador, a média aritmética $(\hat{\mu})$, o desvio-padrão $(\hat{\sigma})$, a proporção de "maus" ajustes $(\hat{\pi})$ e o desvio-padrão associado ao estimador da referida proporção $\left(\hat{\sigma}_{\hat{\pi}}\right)$.

Sobre a classificação de ajustamentos insatisfatórios, foram inicialmente considerados os valores de referência a seguir: para SRMR e RMSEA, os resultados apontam "mau" ajuste quando estão contidos no intervalo ]0,08; $+\infty[$; e para NFI, IFI, TLI/NNFI, CFI, RNI, GFI e AGFI, se pertencem ao subconjunto ] $-\infty ; 0,95$ [ (Hooper et al., 2008). No entanto, as maneiras de qualificar o que é irregular são dignas de adaptações para diferentes tamanhos de amostra e quantidades de variáveis.

Em alguns momentos, foi necessário processar repetidas vezes até que as mil estimativas de 
cada índice e parâmetro fossem calculadas. Para o presente estudo, a ocorrência dessa dificuldade computacional já era prevista, uma vez que se sabe que outliers podem ser responsáveis pela não convergência de métodos iterativos e também resultar na obtenção de valores negativos para variâncias amostrais, pois a estimação das mesmas é assintótica em MEEs (Marôco, 2010).

$\mathrm{Na}$ prática, costuma-se eliminar os outliers para fazer com que o procedimento convirja e as estimativas das variâncias sejam positivas, mas essa ação obviamente impediria a condução deste trabalho, uma vez que aqui se pretende avaliar o impacto de tais pontos em amostras. Além disso, a exclusão de unidades amostrais não é incentivada, especialmente no caso do pesquisador possuir uma amostra "pequena". Embora alguns métodos robustos de estimação tomem o "melhor" subconjunto de uma amostra para computar estimativas, os resultados fornecidos valem para toda a amostra, ou seja, nada é excluído. No âmbito da Análise de Regressão, Paula (2013) orienta que todos os pontos atípicos — influentes, localmente influentes ou de alavanca — sejam mantidos, se não provocarem mudanças na análise inferencial, i.e., quando as conclusões obtidas a partir dos níveis descritivos ${ }^{1}$ das variáveis forem as mesmas com ou sem os pontos contaminados, a um nível de significância fixo.

Para contornar a situação, foi estabelecido que as amostras geradas devem atender a condição de positividade das estimativas das variâncias $\hat{\phi}_{i, i}, \hat{\psi}_{i, i}, \hat{\theta}_{i, i}^{(\boldsymbol{\varepsilon})}$ e $\hat{\theta}_{i, i}^{(\boldsymbol{\delta})}(i \in\{1, \ldots, p+q\})$, o que fez com que o método convergisse muito mais vezes e o tempo de execução das simulações aumentasse.

Esse problema da dificuldade na convergência em amostras contaminadas por outliers pode ser mais ou menos sofrível de acordo com o software adotado, no sentido de alguns terem desempenho pior ou melhor que outros, abortando o processo com uma frequência maior ou menor.

Nos últimos anos, usuários da técnica de Modelagem de Equações Estruturais e outras correlatas estão utilizando cada vez mais o Mplus (Muthén e Muthén, 1998-2010) em estudos², devido ao bom desempenho deste software estatístico. No entanto, a presente obra optou pelo ambiente computacional R 2.15.2 (R Core Team, 2012), por conta do mesmo ser livre e gratuito, pela sua popularidade no meio acadêmico e por suas versões anteriores já terem sido adotadas em textos alinhados a este: em uma dissertação sobre MEEs deste programa de pós-graduação (Bistaffa, 2011) e no artigo que serviu de base para esta pesquisa (Cirillo e Barroso, 2012a). De todo modo, cabe comentar que um recurso bastante útil aos interessados em trabalhar paralelamente com os dois programas mencionados pode ser conferido em Hallquist e Wiley (2013).

Adicionalmente, as seguintes bibliotecas do $\mathrm{R}$ foram empregadas:

- sem, para ajustar MEEs pelo método de Máxima Verossimilhança ${ }^{3}$ (Fox et al., 2013).

- rrcov, para computar as matrizes de covariâncias robustas necessárias para a obtenção dos indicadores robustos (Todorov e Filzmoser, 2009).

O pacote sem do R utiliza a formulação de McDonald e Hartmann (1992) para computar estimativas de parâmetros de MEEs, dada por $\boldsymbol{v}=\boldsymbol{A} \boldsymbol{v}+\boldsymbol{u}$, em que $\boldsymbol{v}$ é um vetor formado por variáveis observadas e latentes, $\boldsymbol{A}$ é uma matriz de coeficientes de regressão e $\boldsymbol{u}$ é um vetor com as correspondentes variáveis de erro associadas às variáveis de $\boldsymbol{v}$ (Fox, 2006).

\footnotetext{
${ }^{1}$ Nível descritivo, valor $p$, $p$-valor e ainda valor de prova são traduções oficiais para $p$-value (Paulino et al., 2011).

${ }^{2}$ Por exemplo, fizemos uso do Mplus 6.12 (Muthén e Muthén, 1998-2010) para aplicar a técnica de Análise de Classes Latentes em uma amostra da região metropolitana de São Paulo, com o objetivo de identificar subtipos depressivos para os gêneros masculino e feminino (Alexandrino-Silva et al., 2013).

${ }^{3}$ Para obter estimativas de parâmetros de um MEE a partir de outros métodos (e.g., Mínimos Quadrados Ordinários e Mínimos Quadrados Generalizados), pode-se recorrer à biblioteca lavaan do R (Rosseel, 2012).
} 
A seguir, os dois estudos de simulação realizados neste trabalho serão apresentados. Os programas desenvolvidos no $\mathrm{R}$ para os dois cenários contemplados estão disponíveis no Apêndice $\mathrm{C}$.

\subsubsection{Cenário 1: Análise Fatorial Confirmatória - muitos parâmetros a estimar}

Nesta subseção, foi tomado um modelo de Análise Fatorial Confirmatória que possui duas variáveis latentes exógenas de variâncias unitárias (i.e., foi especificado que $\phi_{1,1}=\phi_{2,2}=1$, ao invés de estimar estes dois coeficientes) e também correlacionadas, cada uma operacionalizada por quatro variáveis observadas exógenas — todas gaussianas —, conforme descrito na Figura 5.1.

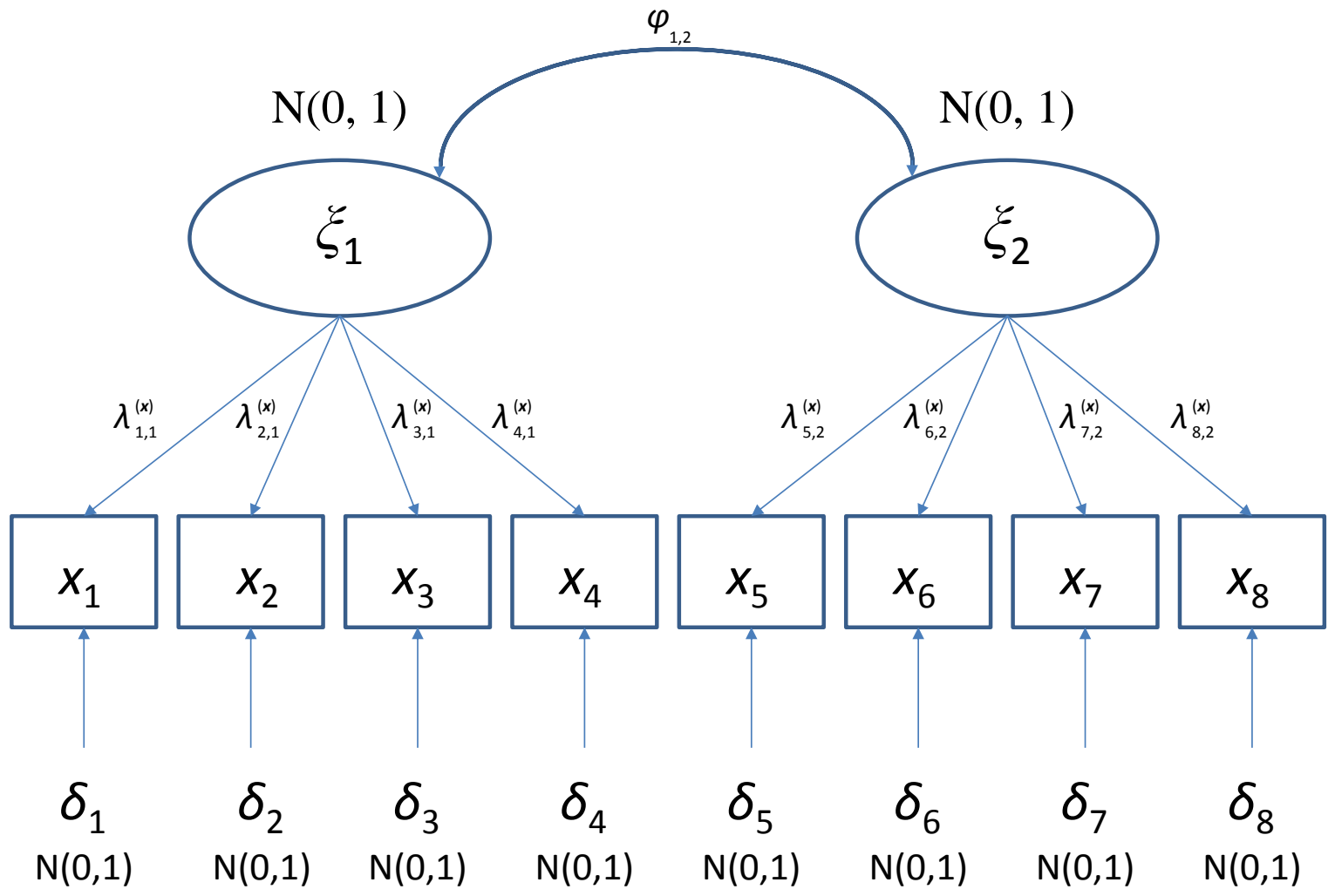

Figura 5.1: Modelo de Análise Fatorial Confirmatória para simulação do Cenário 1.

Esse modelo possui as seguintes equações para o submodelo de mensuração em $\boldsymbol{x}$ :

$$
\begin{aligned}
x_{1} & =\lambda_{1,1}^{(\boldsymbol{x})} \xi_{1}+\delta_{1} \\
x_{2} & =\lambda_{2,1}^{(\boldsymbol{x})} \xi_{1}+\delta_{2} \\
x_{3} & =\lambda_{3,1}^{(\boldsymbol{x})} \xi_{1}+\delta_{3} \\
x_{4} & =\lambda_{4,1}^{(\boldsymbol{x})} \xi_{1}+\delta_{4} \\
x_{5} & =\lambda_{5,2}^{(\boldsymbol{x})} \xi_{2}+\delta_{5} \\
x_{6} & =\lambda_{6,2}^{(\boldsymbol{x})} \xi_{2}+\delta_{6} \\
x_{7} & =\lambda_{7,2}^{(\boldsymbol{x})} \xi_{2}+\delta_{7} \\
x_{8} & =\lambda_{8,2}^{(\boldsymbol{x})} \xi_{2}+\delta_{8}
\end{aligned}
$$


ou, usando matrizes,

$$
\left[\begin{array}{c}
x_{1} \\
x_{2} \\
x_{3} \\
x_{4} \\
x_{5} \\
x_{6} \\
x_{7} \\
x_{8}
\end{array}\right]=\left[\begin{array}{cc}
\lambda_{1,1}^{(\boldsymbol{x})} & 0 \\
\lambda_{2,1}^{(\boldsymbol{x})} & 0 \\
\lambda_{3,1}^{(\boldsymbol{x})} & 0 \\
\lambda_{4,1}^{(\boldsymbol{x})} & 0 \\
0 & \lambda_{5,2}^{(\boldsymbol{x})} \\
0 & \lambda_{6,2}^{(\boldsymbol{x})} \\
0 & \lambda_{7,2}^{(\boldsymbol{x})} \\
0 & \lambda_{8,2}^{(\boldsymbol{x})}
\end{array}\right]\left[\begin{array}{l}
\xi_{1} \\
\xi_{2}
\end{array}\right]+\left[\begin{array}{c}
\delta_{1} \\
\delta_{2} \\
\delta_{3} \\
\delta_{4} \\
\delta_{5} \\
\delta_{6} \\
\delta_{7} \\
\delta_{8}
\end{array}\right]
$$

com

e

$$
\boldsymbol{\Theta}_{\boldsymbol{\delta}}=\left[\begin{array}{cccccccc}
\theta_{1,1}^{(\boldsymbol{\delta})} & 0 & 0 & 0 & 0 & 0 & 0 & 0 \\
0 & \theta_{2,2}^{(\boldsymbol{\delta})} & 0 & 0 & 0 & 0 & 0 & 0 \\
0 & 0 & \theta_{3,3}^{(\boldsymbol{\delta})} & 0 & 0 & 0 & 0 & 0 \\
0 & 0 & 0 & \theta_{4,4}^{(\boldsymbol{\delta})} & 0 & 0 & 0 & 0 \\
0 & 0 & 0 & 0 & \theta_{5,5}^{(\boldsymbol{\delta})} & 0 & 0 & 0 \\
0 & 0 & 0 & 0 & 0 & \theta_{6,6}^{(\boldsymbol{\delta})} & 0 & 0 \\
0 & 0 & 0 & 0 & 0 & 0 & \theta_{7,7}^{(\boldsymbol{\delta})} & 0 \\
0 & 0 & 0 & 0 & 0 & 0 & 0 & \theta_{8,8}^{(\boldsymbol{\delta})}
\end{array}\right]
$$

$$
\boldsymbol{\Phi}=\left[\begin{array}{cc}
1 & \phi_{1,2} \\
\phi_{2,1} & 1
\end{array}\right]
$$

Para efeitos de comparação, arbitrou-se que: $\lambda_{1,1}^{(\boldsymbol{x})}=0,3, \lambda_{2,1}^{(\boldsymbol{x})}=0,6, \lambda_{3,1}^{(\boldsymbol{x})}=0,9, \lambda_{4,1}^{(\boldsymbol{x})}=1,2$, $\lambda_{5,2}^{(\boldsymbol{x})}=-0,7, \lambda_{6,2}^{(\boldsymbol{x})}=1,8, \lambda_{7,2}^{(\boldsymbol{x})}=0,5, \lambda_{8,2}^{(\boldsymbol{x})}=-0,2, \phi_{1,2}=\phi_{2,1}=-0,20$ e $\theta_{1,1}^{(\boldsymbol{\delta})}=\theta_{2,2}^{(\boldsymbol{\delta})}=\cdots=\theta_{8,8}^{(\boldsymbol{\delta})}=1$.

Para contaminar as variáveis observadas exógenas $x_{1}, x_{2}, x_{3}, x_{4}, x_{5}, x_{6}, x_{7}$ e $x_{8}, \alpha \cdot n$ valores de $\delta_{1}, \delta_{2}, \delta_{3}, \delta_{4}, \delta_{5}, \delta_{6}, \delta_{7}$ e $\delta_{8}$ foram substituídos por outliers, respectivamente. No que tange aos desvios de simetria, $\alpha \cdot n$ pontos de cada uma das oito variáveis de erro foram gerados a partir da lei Exponencial de média unitária, pela função $\operatorname{rexp}(\alpha \cdot n, 1)$ do $\mathrm{R}$; quanto aos desvios de curtose, as gerações foram similarmente feitas a partir da lei $t$-Student com cinco graus de liberdade, usando a função $r t(\alpha \cdot n, 5)$ do R. Este plano encontra-se sumarizado na Tabela 5.1.

Tabela 5.1: Esquema para a geração de outliers no Cenário 1.

\begin{tabular}{c|c|c|c}
\hline$n$ & $\alpha$ & $\alpha \cdot n$ & Variáveis perturbadas \\
\hline \hline 100 & 0,05 & 5 & $\delta_{1}$ \\
\hline 100 & 0,25 & 25 & $\delta_{2}$ \\
\hline 200 & 0,05 & 10 & $\delta_{3}$ \\
\hline 200 & 0,25 & 50 & $\delta_{4}$ \\
\hline 300 & 0,05 & 15 & $\delta_{5}$ \\
\hline 300 & 0,25 & 75 & $\delta_{6}$ \\
\hline 500 & 0,05 & 25 & $\delta_{7}$ \\
\hline 500 & 0,25 & 125 & $\delta_{8}$ \\
\hline
\end{tabular}

O programa do Cenário $1 \underline{\text { sem outliers }}(\alpha=0)$ e com $n=500$ está disponível na Seção C.1 do Apêndice C. No referido código, os sinais das médias de estimativas de $\lambda_{5,2}^{(\boldsymbol{x})}, \lambda_{6,2}^{(\boldsymbol{x})}, \lambda_{7,2}^{(\boldsymbol{x})}, \lambda_{8,2}^{(\boldsymbol{x})}$ e $\phi_{1,2}$ aparecem invertidos em relação aos especificados no início desta subseção, mas isso não caracteriza um problema, uma vez que se sabe que: se $\boldsymbol{w}$ é um vetor próprio, então $-\boldsymbol{w}$ também o é. 


\section{Resultados e discussão}

As estimativas dos parâmetros do MEE da Figura 5.1 em condições regulares (Seção 2.6) estão apresentadas na Tabela 5.2. Observa-se que os valores médios vão se aproximando do verdadeiro e os desvios-padrão vão diminuindo à medida que o tamanho da amostra aumenta.

Quando $n=100$, as médias das estimativas dos parâmetros $\lambda_{5,2}^{(\boldsymbol{x})}, \lambda_{6,2}^{(\boldsymbol{x})}, \lambda_{7,2}^{(\boldsymbol{x})}, \lambda_{8,2}^{(\boldsymbol{x})}$ e $\phi_{1,2}$ apresentam um desvio considerável em relação aos fixados para comparação - uma evidência de que esse tamanho de amostra não é o ideal para a estimação desses dezessete parâmetros. Pela análise da Tabela 5.3, isso refletiu no desempenho médio dos indicadores NFI, GFI $\mathrm{MV}$, GFI $\mathrm{MV}$, $\mathrm{GFI}_{\mathrm{MV}}^{\mathrm{CDM}}$, $\mathrm{AGFI}_{\mathrm{MV}}, \mathrm{AGFI}_{\mathrm{MV}}^{\mathrm{GKO}}, \mathrm{AGFI}_{\mathrm{MV}}^{\mathrm{EVM}}$ e $\mathrm{AGFI}_{\mathrm{MV}}^{\mathrm{CDM}}$, que detectaram essas distorções.

A melhoria de $\mathrm{GFI}_{\mathrm{MV}}^{\mathrm{EVM}}$ e $\mathrm{GFI}_{\mathrm{MV}}^{\mathrm{CDM}}$ em relação ao usual $\mathrm{GFI}_{\mathrm{MV}}$ era esperada para o tamanho de amostra $n=100$. Cirillo e Barroso (2012a) usaram as quantidades

$$
P_{\mathrm{MQO}}^{\mathrm{EVM}}=\left(\frac{\mathrm{GFI}_{\mathrm{MQO}}^{\mathrm{EVM}}-\mathrm{GFI}_{\mathrm{MQO}}}{\mathrm{GFI}_{\mathrm{MQO}}}\right) \times 100 \%
$$

$\mathrm{e}$

$$
P_{\mathrm{MQO}}^{\mathrm{CDM}}=\left(\frac{\mathrm{GFI}_{\mathrm{MQO}}^{\mathrm{CDM}}-\mathrm{GFI}_{\mathrm{MQO}}}{\mathrm{GFI}_{\mathrm{MQO}}}\right) \times 100 \%
$$

para mostrar que esses dois índices têm desempenho superior ao tradicional nos casos em que $n \leq 200$ e $\alpha \leq 0,30$. Em geral, o uso de GFI $_{M V}$ não vem sendo recomendado (Sharma et al., 2005).

Quando $200 \leq n \leq 300$, as médias de NFI e $\mathrm{AGFI}_{\mathrm{MV}}^{\mathrm{GKO}}$ estão entre 0,90 e 0,95 , o que não quer dizer que eles erroneamente estão sugerindo inadequabilidade. Isso tem a ver com os problemas de cometer o Erro do Tipo I (i.e., a rejeição incorreta de um MEE aceitável) ao seguir à risca uma lista de valores de corte (Marsh et al., 2004), no sentido de que a classificação de "mau" ajuste em ] $-\infty ; 0,95[$ não deve ser tão rigorosa, sobretudo ao tomar nota de que não há na literatura uma recomendação precisa sobre qual é o limite superior correto para aquele intervalo.

Recorrendo às interpretações de valores descritas nas Subseções 4.1.3, 4.1.8 e 4.1.9, apenas os resultados referentes ao ajuste com tamanho de amostra $n=100$ receberam uma má avaliação pelo NFI. Para $\mathrm{AGFI}_{\mathrm{MV}}^{\mathrm{GKO}}$, os ajustes foram conceituados como segue: sofríveis, para $n=100$; bons, para $100<n<500$; e muito bons, para $n=500$. Assim, percebe-se que ambas as medidas estão dentro do esperado, ao estudar suas escalas particulares.

Contaminando os dados com $5 \%$ de valores da lei Exponencial de esperança matemática unitária, as médias das estimativas dos parâmetros sofrem perceptíveis afetações em relação aos parâmetros, em todos os quatro tamanhos de amostra contemplados. Pela Tabela 5.4, observa-se que a maioria das médias das estimativas foi maior que os valores fixados para comparação.

No que diz respeito aos indicadores, a Tabela 5.5 ilustra que o desempenho dos mesmos é fortemente influenciado pela dimensão do conjunto de dados, o que sugere cuidados quanto ao risco de julgar razoável um MEE mal ajustado em virtude da existência de outliers.

O indicador RMSEA apresentou mau desempenho para todos os tamanhos de amostra.

Apesar de os métodos robustos S e MM possuírem alto ponto de ruptura e serem altamente eficientes sob normalidade, as modificações $\mathrm{GFI}_{\mathrm{MV}}^{\mathrm{S}}$ e $\mathrm{GFI}_{\mathrm{MV}}^{\mathrm{MM}}$ não são recomendadas em nenhum tamanho de amostra, pois elas também levariam o pesquisador a equivocadamente crer que seu modelo está bem ajustado. As correspondentes versões ajustadas, $\mathrm{AGFI}_{\mathrm{MV}}^{\mathrm{S}}$ e $\mathrm{AGFI}_{\mathrm{MV}}^{\mathrm{MM}}$, tiveram desempenho inferior a $\mathrm{AGFI}_{\mathrm{MV}}$ e até mesmo também a $\mathrm{GFI}_{\mathrm{MV}}$. 
Para $n=100$, as médias das medidas SRMR, NFI, IFI, NNFI, CFI, RNI, GFI $\mathrm{MV}_{\text {, GFI }}^{\mathrm{GKO}}$, $\mathrm{GFI}_{\mathrm{MV}}^{\mathrm{EVM}}$ e $\mathrm{GFI}_{\mathrm{MV}}^{\mathrm{CDM}}$ e todas as seis versões do AGFI apontam que o MEE está mal ajustado, tendo

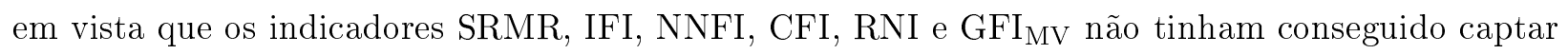
as distorções resultantes tão apenas pela baixa quantidade de observações em relação ao número de parâmetros estimados nas amostras sem outliers.

Para $n>100$, a maioria dos indicadores incorretamente sugere que o modelo está bem ou muito bem ajustado, em virtude de todos eles serem sensíveis ao número de observações - são inflacionados à medida que o tamanho da amostra aumenta. Entretanto, uma forma de contornar essa dificuldade vem a partir da orientação de analisar mutuamente os índices (Bollen, 1989), ao invés de comparar os resultados das medidas somente com seus pontos de corte.

Tomando o valor $n=500$, os índices NFI e $\mathrm{AGFI}_{\mathrm{MV}}^{\mathrm{GKO}}$ possuem médias inferiores a 0,93 para este caso de a amostra estar contaminada com 5\% de valores da lei Exponencial de média unitária, enquanto suas médias estão acima de 0,95 quando não há outliers, com base na comparação entre a Tabela 5.3 e a Tabela 5.5. Em palavras, essa frequência de pontos atípicos não faria com que NFI e $\mathrm{AGFI}_{\mathrm{MV}}^{\mathrm{GKO}}$ erroneamente entrassem na zona de ajustes muito bons, o que já ocorre com as medidas SRMR, RMSEA e todas as seis versões do GFI. Como as médias dos índices IFI, CFI, RNI e $\mathrm{AGFI}_{\mathrm{MV}}$ estão em torno de 0,945, um ligeiro incremento no tamanho amostral faria com que também eles inadequadamente qualificassem muito bem os ajustes.

Quando $200 \leq n \leq 300$, as médias de NFI conseguem informar que os ajustes estão sofríveis e, pela análise das estimativas da proporção, mais de $98 \%$ das mil replicações simuladas não receberam o rótulo de muito boas. Sob contaminação, os valores médios de $\mathrm{AGFI}_{\mathrm{MV}}^{\mathrm{GKO}}$ estão abaixo de 0,92; e sob condições regulares, as médias das estimativas de $\mathrm{AGFI}_{\mathrm{MV}}^{\mathrm{GKO}}$ estão acima de 0,92. Para IFI, CFI, RNI e AGFI $I_{M V}$, a proporção de $5 \%$ de mistura de distribuições faz com que suas médias sejam maiores ou iguais a 0,94 , portanto pouco informativas, pelo que transitam entre os agrupamentos bom e muito bom.

O indicador NNFI parecia seguir a mesma linha de raciocínio dos indicadores NFI e $\mathrm{AGFI}_{\mathrm{MV}}^{\mathrm{GKO}}$, mas apresentou resultados com uma inclinação oposta quando $n=200$.

Aumentando a fração de contaminação de valores da lei Exponencial de média unitária para 25\%, os valores médios das estimativas dos parâmetros são severamente afetados, pelo que se constata na Tabela 5.6. Como se trata de um alto impacto, todos os indicadores da Tabela 5.7 foram realistas quanto ao diagnóstico de ajuste ruim, independentemente da quantidade de observações. Contudo, cabe notar que esses achados têm mais importância teórica, uma vez que essa elevada proporção de outliers tende a ser incomum em dados reais.

Ao passar a lidar com a fração de $5 \%$ de contaminação proveniente da lei $t$-Student com cinco graus de liberdade, é possível averiguar na Tabela 5.8 que as estimativas dos parâmetros foram afetadas em decorrência dessa mistura distribucional, embora os desvios não tenham sido tão acentuados quanto aos observados na Tabela 5.4, referente à substituição de $5 \%$ dos pontos por valores da lei Exponencial de média unitária.

Através da apuração dos resultados dos indicadores fornecidos na Tabela 5.9, mais uma vez a influência do tamanho da amostra foi detectada. Além disso, foi notada a incapacidade da maioria dos índices quanto ao poder de detecção de problemas nas estimativas ocasionados por pequenas proporções de distribuição com excesso de curtose, mesmo quando a quantidade de observações no conjunto de dados é baixa. 
Ao tamanho de amostra $n=100$, as medidas NFI, $\mathrm{GFI}_{\mathrm{MV}}^{\mathrm{GKO}}, \mathrm{GFI}_{\mathrm{MV}}^{\mathrm{EVM}}$ e $\mathrm{GFI}_{\mathrm{MV}}^{\mathrm{CDM}}, \mathrm{AGFI}_{\mathrm{MV}}^{\mathrm{GKO}}$, $\mathrm{AGFI}_{\mathrm{MV}}^{\mathrm{EVM}}$ e $\mathrm{AGFI}_{\mathrm{MV}}^{\mathrm{CDM}}$ mostraram-se efetivas para indicar maus ajustes, pelo que as médias de suas estimativas estão abaixo de 0,90. Era esperado que o desempenho de $\mathrm{GFI}_{\mathrm{MV}}^{\mathrm{EVM}}$ e $\mathrm{GFI}_{\mathrm{MV}}^{\mathrm{CDM}}$ fosse superior ao de $\mathrm{GFI}_{\mathrm{Mv}}$ para essa quantidade de observações (Cirillo e Barroso, 2012a).

Para $n>100$, somente os índices NFI e $\mathrm{AGFI}_{\mathrm{MV}}^{\mathrm{GKO}}$ conseguem aconselhar o pesquisador de que possa haver problemas nos dados relacionados à curtose e aos ajustes de um MEE, pelo que suas médias em geral estão abaixo de 0,93, enquanto que as médias das estimativas obtidas para os demais indicadores são superiores a 0,95 já para $n=200$.

Sobre o que foi revisado no início da Seção 4.1, afastamentos na curtose quebram uma das assunções necessárias para aplicar o teste Qui-Quadrado de hipóteses. Assim, a análise das medidas NFI e $\mathrm{AGFI}_{\mathrm{MV}}^{\mathrm{GKO}}$ é sugerida para avaliar essa premissa.

Crescendo a contaminação pela lei $t$-Student com cinco graus de liberdade para o percentual de 25\%, as médias das estimativas dos parâmetros foram fortemente afetadas (Tabela 5.10). Esses resultados aberrantes foram identificados por todos os indicadores aqui contemplados, nos quatro tamanhos de amostra adotados (Tabela 5.11). No entanto, provavelmente um analista de dados não irá se deparar com esta situação na prática.

Pelo que foi exposto acerca dos resultados encontrados para amostras de tamanhos intermediários $(100<n \leq 300)$, cabe reforçar a atenção quanto ao ponto de corte dos índices que sugerem melhores resultados para maiores valores (quando a aproximação à unidade é desejável, na maioria dos casos). Em condições normais, valores acima de 0,90 fornecidos por essas medidas parecem adequados para qualificar ajustes de MEEs como sendo bons ou muito bons. Sob irregularidades relacionadas a pequenas - porém impactantes - frações de contaminação, valores de NFI e $\mathrm{AGFI}_{\mathrm{MV}}^{\mathrm{GKO}}$ abaixo de 0,93 levantam suspeitas sobre contaminação. Isso evidencia que o entendimento sobre valores de NFI e $\mathrm{AGFI}_{\mathrm{MV}}^{\mathrm{GKO}}$ que estejam próximos ao ponto médio da faixa ]0,90; 0,93[ requer suporte de outros tipos de análises.

Ainda sobre o efeito de baixas frações de contaminação $(\alpha=0,05)$ refletido nos indicadores NFI e $\mathrm{AGFI}_{\mathrm{MV}}^{\mathrm{GKO}}$, tem-se: se a dimensão da amostra é "pequena" $(n \leq 100)$, valores abaixo de 0,90 sugerem problemas relacionados a outliers ou à limitada quantidade de observações; e se o tamanho amostral é "grande" ( $n>300)$, valores abaixo de 0,95 sugerem que as estimativas estejam afetadas por valores atípicos. De qualquer forma, a recomendação de reforçar esses achados combinando com os resultados fornecidos por outras técnicas deve sempre ser mantida.

Examinando NFI e $\mathrm{AGFI}_{\mathrm{MV}}^{\mathrm{GKO}}$ entre si, tem-se que o primeiro é mais preciso, pelo que os desviospadrão de suas estimativas são menores. 
Tabela 5.2: Estatísticas dos mil valores obtidos via simulação para as estimativas dos parâmetros do MEE descrito pela Figura 5.1, por tamanho de amostra e com fração de contaminação zero.

\begin{tabular}{|c|c|c|c|c|c|}
\hline \multirow{3}{*}{$\begin{array}{c}\text { Parâmetro } \\
\lambda_{11}^{(\boldsymbol{x})}\end{array}$} & \multirow{3}{*}{$\begin{array}{c}\text { Referência } \\
\quad\{0,3\}\end{array}$} & \multicolumn{2}{|c|}{$n=100$} & \multicolumn{2}{|c|}{$n=200$} \\
\hline & & $\hat{\mu}_{\hat{\theta}}$ & $\hat{\sigma}_{\hat{\theta}}$ & $\hat{\mu}_{\hat{\theta}}$ & $\hat{\sigma}_{\hat{\theta}}$ \\
\hline & & 0,304 & 0,119 & 0,300 & 0,084 \\
\hline$\lambda_{2,1}^{(\boldsymbol{x})}$ & $\{0,6\}$ & 0,602 & 0,133 & 0,597 & 0,096 \\
\hline$\lambda_{3,1}^{(\boldsymbol{x})}$ & $\{0,9\}$ & 0,910 & 0,157 & 0,904 & 0,112 \\
\hline$\lambda_{4,1}^{(\boldsymbol{x})}$ & $\{1,2\}$ & 1,190 & 0,190 & 1,198 & 0,128 \\
\hline$\lambda_{5,2}^{(\boldsymbol{x})}$ & $\{-0,7\}$ & $-0,570$ & 0,506 & $-0,695$ & 0,242 \\
\hline$\lambda_{6,2}^{(\boldsymbol{x})}$ & $\{1,8\}$ & 1,459 & 1,014 & 1,753 & 0,493 \\
\hline$\lambda_{7,2}^{(x)}$ & $\{0,5\}$ & 0,426 & 0,335 & 0,495 & 0,172 \\
\hline$\lambda_{8,2}^{(\boldsymbol{x})}$ & $\{-0,2\}$ & $-0,168$ & 0,173 & $-0,198$ & 0,102 \\
\hline$\phi_{1,2}$ & $\{-0,2\}$ & $-0,162$ & 0,190 & $-0,193$ & 0,106 \\
\hline$\theta_{1,1}^{(\boldsymbol{\delta})}$ & $\{1,0\}$ & 0,990 & 0,146 & 0,990 & 0,102 \\
\hline$\theta_{2,2}^{(\boldsymbol{\delta})}$ & $\{1,0\}$ & 0,987 & 0,174 & 0,994 & 0,116 \\
\hline$\theta_{3,3}^{(\tilde{\boldsymbol{\delta}})}$ & $\{1,0\}$ & 0,967 & 0,234 & 0,979 & 0,166 \\
\hline$\theta_{4,4}^{(\boldsymbol{\delta})}$ & $\{1,0\}$ & 0,988 & 0,350 & 0,987 & 0,255 \\
\hline$\theta_{5,5}^{(\boldsymbol{\delta})}$ & $\{1,0\}$ & 0,929 & 0,191 & 0,973 & 0,126 \\
\hline$\theta_{6,6}^{(\boldsymbol{\delta})}$ & $\{1,0\}$ & 1,226 & 0,673 & 1,058 & 0,520 \\
\hline$\theta_{7,7}^{(\boldsymbol{\delta})}$ & $\{1,0\}$ & 0,969 & 0,163 & 0,985 & 0,104 \\
\hline \multirow[t]{2}{*}{$\theta_{8,8}^{(\boldsymbol{\delta})}$} & $\{1,0\}$ & 0,986 & 0,146 & 0,998 & 0,099 \\
\hline & & \multicolumn{2}{|c|}{$n=300$} & \multicolumn{2}{|c|}{$n=500$} \\
\hline Parâmetro & Referência & $\hat{\mu}_{\hat{\theta}}$ & $\hat{\sigma}_{\hat{\theta}}$ & $\hat{\mu}_{\hat{\theta}}$ & $\hat{\sigma}_{\hat{\theta}}$ \\
\hline$\lambda_{1,1}^{(\boldsymbol{x})}$ & $\{0,3\}$ & 0,296 & 0,069 & 0,300 & 0,053 \\
\hline$\lambda_{2,1}^{(\boldsymbol{x})}$ & $\{0,6\}$ & 0,601 & 0,076 & 0,601 & 0,057 \\
\hline$\lambda_{3,1}^{(\boldsymbol{x})}$ & $\{0,9\}$ & 0,902 & 0,090 & 0,902 & 0,069 \\
\hline$\lambda_{4,1}^{(\boldsymbol{x})}$ & $\{1,2\}$ & 1,200 & 0,109 & 1,202 & 0,082 \\
\hline$\lambda_{5,2}^{(x)}$ & $\{-0,7\}$ & $-0,712$ & 0,145 & $-0,712$ & 0,067 \\
\hline$\lambda_{6,2}^{(\boldsymbol{x})}$ & $\{1,8\}$ & 1,797 & 0,296 & 1,844 & 0,122 \\
\hline$\lambda_{7,2}^{(\boldsymbol{x})}$ & $\{0,5\}$ & 0,508 & 0,109 & 0,510 & 0,056 \\
\hline$\lambda_{8,2}^{(\boldsymbol{x})}$ & $\{-0,2\}$ & $-0,202$ & 0,072 & $-0,206$ & 0,052 \\
\hline$\phi_{1,2}$ & $\{-0,2\}$ & $-0,197$ & 0,079 & $-0,195$ & 0,057 \\
\hline$\theta_{1,1}^{(\boldsymbol{\delta})}$ & $\{1,0\}$ & 0,995 & 0,086 & 0,996 & 0,064 \\
\hline$\theta_{2,2}^{(\boldsymbol{\delta})}$ & $\{1,0\}$ & 0,996 & 0,098 & 0,997 & 0,075 \\
\hline$\theta_{3,3}^{(\boldsymbol{\delta})}$ & $\{1,0\}$ & 0,989 & 0,132 & 0,994 & 0,104 \\
\hline$\theta_{4,4}^{(\boldsymbol{\delta})}$ & $\{1,0\}$ & 0,992 & 0,209 & 0,994 & 0,156 \\
\hline$\theta_{5,5}^{(\boldsymbol{\delta})}$ & $\{1,0\}$ & 0,980 & 0,111 & 1,000 & 0,085 \\
\hline$\theta_{6,6}^{(\boldsymbol{\delta})}$ & $\{1,0\}$ & 1,045 & 0,462 & 0,958 & 0,379 \\
\hline$\theta_{7,7}^{(\boldsymbol{\delta})}$ & $\{1,0\}$ & 0,988 & 0,091 & 1,000 & 0,070 \\
\hline$\theta_{8.8}^{(\boldsymbol{\delta})}$ & $\{1,0\}$ & 0,996 & 0,083 & 0,997 & 0,065 \\
\hline
\end{tabular}


Tabela 5.3: Estatísticas dos mil valores obtidos via simulação para os indicadores clássicos e robustos do MEE descrito pela Figura 5.1, por tamanho de amostra e com fração de contaminação zero.

\begin{tabular}{|c|c|c|c|c|c|c|c|c|c|}
\hline \multirow[b]{2}{*}{ Índice } & \multirow[b]{2}{*}{ Referência } & \multicolumn{4}{|c|}{$n=100$} & \multicolumn{4}{|c|}{$n=200$} \\
\hline & & $\hat{\mu}$ & $\hat{\sigma}$ & $\hat{\pi}$ & $\hat{\sigma}_{\hat{\pi}}$ & $\hat{\mu}$ & $\hat{\sigma}$ & $\hat{\pi}$ & $\hat{\sigma}_{\hat{\pi}}$ \\
\hline SRMR & {$[0 ; 0,08[$} & 0,062 & 0,012 & 0,063 & 0,243 & 0,044 & 0,008 & 0,000 & 0,000 \\
\hline RMSEA & {$[0 ; 0,08[$} & 0,023 & 0,028 & 0,040 & 0,196 & 0,016 & 0,020 & 0,000 & 0,000 \\
\hline NFI & ] $0,90 ; 1]$ & 0,860 & 0,044 & 0,997 & 0,055 & 0,923 & 0,025 & 0,878 & 0,327 \\
\hline IFI & ] $0,90 ; 1]$ & 1,002 & 0,053 & 0,158 & 0,365 & 1,000 & 0,028 & 0,046 & 0,210 \\
\hline NNFI & ] $0,90 ; 1]$ & 1,004 & 0,086 & 0,255 & 0,436 & 1,000 & 0,042 & 0,121 & 0,326 \\
\hline CFI & ] $0,90 ; 1]$ & 0,978 & 0,034 & 0,178 & 0,383 & 0,989 & 0,018 & 0,052 & 0,222 \\
\hline RNI & ] $0,90 ; 1]$ & 1,003 & 0,059 & 0,178 & 0,383 & 1,000 & 0,029 & 0,052 & 0,222 \\
\hline $\mathrm{GFI}_{\mathrm{MV}}$ & ] $0,90 ; 1]$ & 0,956 & 0,013 & 0,316 & 0,465 & 0,977 & 0,007 & 0,001 & 0,032 \\
\hline $\mathrm{GFI}_{\mathrm{MV}}^{\mathrm{GKO}}$ & ] $0,90 ; 1]$ & 0,938 & 0,042 & 0,508 & 0,500 & 0,959 & 0,022 & 0,273 & 0,446 \\
\hline $\mathrm{GFI}_{\mathrm{MV}}^{\mathrm{EVM}}$ & $0,90 ; 1]$ & 0,941 & 0,028 & 0,548 & 0,498 & 0,976 & 0,011 & 0,022 & 0,147 \\
\hline $\mathrm{GFI}_{\mathrm{MV}}^{\mathrm{CDM}}$ & ] $0,90 ; 1]$ & 0,938 & 0,027 & 0,619 & 0,486 & 0,979 & 0,009 & 0,012 & 0,109 \\
\hline $\mathrm{GFI}_{\mathrm{MV}}^{\mathrm{S}}$ & ] $0,90 ; 1]$ & 0,977 & 0,009 & 0,009 & 0,094 & 0,989 & 0,004 & 0,000 & 0,000 \\
\hline $\mathrm{GFI}_{\mathrm{MV}}^{\mathrm{MM}}$ & ] $0,90 ; 1]$ & 0,982 & 0,007 & 0,001 & 0,032 & 0,991 & 0,004 & 0,000 & 0,000 \\
\hline $\mathrm{AGFI}_{\mathrm{MV}}$ & ] $0,90 ; 1]$ & 0,916 & 0,025 & 0,926 & 0,262 & 0,956 & 0,014 & 0,285 & 0,452 \\
\hline $\mathrm{AGFI}_{\mathrm{MV}}^{\mathrm{GKO}}$ & ] $0,90 ; 1]$ & 0,883 & 0,080 & 0,874 & 0,332 & 0,922 & 0,041 & 0,721 & 0,449 \\
\hline $\mathrm{AGFI}_{\mathrm{MV}}^{\mathrm{EVM}}$ & ] $0,90 ; 1]$ & 0,888 & 0,053 & 0,951 & 0,216 & 0,955 & 0,020 & 0,304 & 0,460 \\
\hline $\mathrm{AGFI}_{\mathrm{MV}}^{\mathrm{CDM}}$ & ] $0,90 ; 1]$ & 0,883 & 0,051 & 0,961 & 0,194 & 0,961 & 0,017 & 0,203 & 0,402 \\
\hline $\mathrm{AGFI}_{\mathrm{MV}}^{\mathrm{S}}$ & ] $0,90 ; 1]$ & 0,957 & 0,017 & 0,282 & 0,450 & 0,978 & 0,008 & 0,006 & 0,077 \\
\hline \multirow[t]{2}{*}{$\mathrm{AGFI}_{\mathrm{MV}}^{\mathrm{MM}}$} & ] $0,90 ; 1]$ & 0,966 & 0,013 & 0,119 & 0,324 & 0,983 & 0,007 & 0,001 & 0,032 \\
\hline & & \multicolumn{4}{|c|}{$n=300$} & \multicolumn{4}{|c|}{$n=500$} \\
\hline Índice & Referência & $\hat{\mu}$ & $\hat{\sigma}$ & $\hat{\pi}$ & $\hat{\sigma}_{\hat{\pi}}$ & $\hat{\mu}$ & $\hat{\sigma}$ & $\hat{\pi}$ & $\hat{\sigma}_{\hat{\pi}}$ \\
\hline SRMR & {$[0 ; 0,08[$} & 0,036 & 0,007 & 0,000 & 0,000 & 0,028 & 0,005 & 0,000 & 0,000 \\
\hline RMSEA & {$[0 ; 0,08[$} & 0,014 & 0,017 & 0,000 & 0,000 & 0,010 & 0,013 & 0,000 & 0,000 \\
\hline NFI & ] $0,90 ; 1]$ & 0,946 & 0,017 & 0,552 & 0,498 & 0,967 & 0,011 & 0,070 & 0,255 \\
\hline IFI & ] $0,90 ; 1]$ & 0,999 & 0,018 & 0,008 & 0,089 & 1,000 & 0,011 & 0,002 & 0,045 \\
\hline NNFI & ] $0,90 ; 1]$ & 0,999 & 0,028 & 0,055 & 0,228 & 1,000 & 0,017 & 0,007 & 0,083 \\
\hline CFI & ] $0,90 ; 1]$ & 0,992 & 0,012 & 0,011 & 0,104 & 0,995 & 0,007 & 0,002 & 0,045 \\
\hline RNI & ] $0,90 ; 1]$ & 0,999 & 0,019 & 0,011 & 0,104 & 1,000 & 0,012 & 0,002 & 0,045 \\
\hline $\mathrm{GFI}_{\mathrm{MV}}$ & ] $0,90 ; 1]$ & 0,984 & 0,005 & 0,000 & 0,000 & 0,991 & 0,003 & 0,000 & 0,000 \\
\hline $\mathrm{GFI}_{\mathrm{MV}}^{\mathrm{GKO}}$ & ] $0,90 ; 1]$ & 0,967 & 0,016 & 0,137 & 0,344 & 0,974 & 0,011 & 0,043 & 0,203 \\
\hline $\mathrm{GFI}_{\mathrm{MV}}^{\mathrm{EVM}}$ & ] $0,90 ; 1]$ & 0,985 & 0,007 & 0,002 & 0,045 & 0,991 & 0,004 & 0,000 & 0,000 \\
\hline $\mathrm{GFI}_{\mathrm{MV}}^{\mathrm{CDM}}$ & ] $0,90 ; 1]$ & 0,988 & 0,005 & 0,002 & 0,045 & 0,994 & 0,002 & 0,000 & 0,000 \\
\hline $\mathrm{GFI}_{\mathrm{MV}}^{\mathrm{S}}$ & ] $0,90 ; 1]$ & 0,992 & 0,003 & 0,000 & 0,000 & 0,995 & 0,002 & 0,000 & 0,000 \\
\hline $\mathrm{GFI}_{\mathrm{MV}}^{\mathrm{MM}}$ & ] $0,90 ; 1]$ & 0,994 & 0,002 & 0,000 & 0,000 & 0,996 & 0,001 & 0,000 & 0,000 \\
\hline $\mathrm{AGFI}_{\mathrm{MV}}$ & ] $0,90 ; 1]$ & 0,970 & 0,009 & 0,027 & 0,162 & 0,982 & 0,006 & 0,000 & 0,000 \\
\hline $\mathrm{AGFI}_{\mathrm{MV}}^{\mathrm{GKO}}$ & ] $0,90 ; 1]$ & 0,938 & 0,030 & 0,578 & 0,494 & 0,951 & 0,021 & 0,408 & 0,492 \\
\hline $\mathrm{AGFI}_{\mathrm{MV}}^{\mathrm{EV}}$ & ] $0,90 ; 1]$ & 0,972 & 0,013 & 0,051 & 0,220 & 0,984 & 0,008 & 0,008 & 0,089 \\
\hline $\mathrm{AGFI}_{\mathrm{MV}}^{\mathrm{CDM}}$ & ] $0,90 ; 1]$ & 0,977 & 0,010 & 0,016 & 0,126 & 0,988 & 0,005 & 0,000 & 0,000 \\
\hline $\mathrm{AGFI}_{\mathrm{MV}}^{\mathrm{S}}$ & ] $0,90 ; 1]$ & 0,985 & 0,006 & 0,001 & 0,032 & 0,991 & 0,003 & 0,000 & 0,000 \\
\hline $\mathrm{AGFI}_{\mathrm{MV}}^{\mathrm{MM}}$ & ] $0,90 ; 1]$ & 0,988 & 0,005 & 0,000 & 0,000 & 0,993 & 0,003 & 0,000 & 0,000 \\
\hline
\end{tabular}


Tabela 5.4: Estatísticas dos mil valores obtidos via simulação para as estimativas dos parâmetros do MEE descrito pela Figura 5.1, por tamanho de amostra e com fração de 5\% de contaminação proveniente da lei Exponencial de média unitária.

\begin{tabular}{|c|c|c|c|c|c|}
\hline \multirow{3}{*}{$\begin{array}{c}\text { Parâmetro } \\
\lambda_{1.1}^{(\boldsymbol{x})}\end{array}$} & \multirow{3}{*}{$\begin{array}{c}\text { Referência } \\
\quad\{0,3\}\end{array}$} & \multicolumn{2}{|c|}{$n=100$} & \multicolumn{2}{|c|}{$n=200$} \\
\hline & & $\hat{\mu}_{\hat{\theta}}$ & $\hat{\sigma}_{\hat{\theta}}$ & $\hat{\mu}_{\hat{\theta}}$ & $\hat{\sigma}_{\hat{\theta}}$ \\
\hline & & 0,409 & 0,116 & 0,341 & 0,080 \\
\hline$\lambda_{2,1}^{(\boldsymbol{x})}$ & $\{0,6\}$ & 0,666 & 0,122 & 0,646 & 0,087 \\
\hline$\lambda_{3,1}^{(\boldsymbol{x})}$ & $\{0,9\}$ & 0,945 & 0,137 & 0,911 & 0,104 \\
\hline$\lambda_{4,1}^{(\boldsymbol{x})}$ & $\{1,2\}$ & 1,278 & 0,180 & 1,257 & 0,128 \\
\hline$\lambda_{5,2}^{(\boldsymbol{x})}$ & $\{-0,7\}$ & $-0,090$ & 0,809 & $-0,576$ & 0,409 \\
\hline$\lambda_{6,2}^{(\boldsymbol{x})}$ & $\{1,8\}$ & 0,519 & 1,511 & 1,591 & 0,951 \\
\hline$\lambda_{7,2}^{(\boldsymbol{x})}$ & $\{0,5\}$ & 0,154 & 0,519 & 0,442 & 0,288 \\
\hline$\lambda_{8,2}^{(x)}$ & $\{-0,2\}$ & $-0,016$ & 0,305 & $-0,148$ & 0,131 \\
\hline$\phi_{1,2}$ & $\{-0,2\}$ & $-0,027$ & 0,277 & $-0,147$ & 0,133 \\
\hline$\theta_{1,1}^{(\boldsymbol{\delta})}$ & $\{1,0\}$ & 0,987 & 0,149 & 0,961 & 0,102 \\
\hline$\theta_{2,2}^{(\tilde{\boldsymbol{d}})}$ & $\{1,0\}$ & 0,950 & 0,160 & 0,944 & 0,116 \\
\hline$\theta_{3,3}^{(\boldsymbol{\delta})}$ & $\{1,0\}$ & 0,935 & 0,216 & 0,958 & 0,154 \\
\hline$\theta_{4.4}^{(\boldsymbol{\delta})}$ & $\{1,0\}$ & 0,917 & 0,327 & 0,919 & 0,257 \\
\hline$\theta_{5,5}^{(\boldsymbol{\delta})}$ & $\{1,0\}$ & 1,042 & 0,370 & 0,994 & 0,133 \\
\hline$\theta_{6,6}^{(\boldsymbol{\delta})}$ & $\{1,0\}$ & 1,969 & 0,997 & 0,947 & 0,528 \\
\hline$\theta_{7,7}^{(\boldsymbol{\delta})}$ & $\{1,0\}$ & 0,949 & 0,179 & 1,015 & 0,103 \\
\hline \multirow[t]{2}{*}{$\theta_{8,8}^{(\boldsymbol{\delta})}$} & $\{1,0\}$ & 1,274 & 0,160 & 1,184 & 0,097 \\
\hline & & \multicolumn{2}{|c|}{$n=300$} & \multicolumn{2}{|c|}{$n=500$} \\
\hline \multirow{3}{*}{$\begin{array}{c}\text { Parâmetro } \\
\lambda_{1,1}^{(\boldsymbol{x})} \\
\lambda_{(\boldsymbol{x})}\end{array}$} & \multirow{2}{*}{$\begin{array}{c}\text { Referência } \\
\qquad\{0,3\}\end{array}$} & $\hat{\mu}_{\hat{\theta}}$ & $\hat{\sigma}_{\hat{\theta}}$ & $\hat{\mu}_{\hat{\theta}}$ & $\hat{\sigma}_{\hat{\theta}}$ \\
\hline & & 0,387 & 0,070 & 0,412 & 0,053 \\
\hline & $\{0,6\}$ & 0,640 & 0,072 & 0,682 & 0,054 \\
\hline$\lambda_{3,1}^{(x)}$ & $\{0,9\}$ & 0,984 & 0,087 & 0,950 & 0,062 \\
\hline$\lambda_{4,1}^{(\boldsymbol{x})}$ & $\{1,2\}$ & 1,203 & 0,100 & 1,217 & 0,074 \\
\hline $\begin{array}{l}\lambda_{5,2}^{(, 1}(\boldsymbol{x}) \\
\text { (x) }\end{array}$ & $\{-0,7\}$ & $-0,641$ & 0,117 & $-0,650$ & 0,080 \\
\hline$\lambda_{6,2}^{(x)}$ & $\{1,8\}$ & 1,892 & 0,215 & 1,923 & 0,168 \\
\hline$\lambda_{7,2}^{(\boldsymbol{x})}$ & $\{0,5\}$ & 0,511 & 0,079 & 0,521 & 0,061 \\
\hline$\lambda_{8,2}^{(\boldsymbol{x})}$ & $\{-0,2\}$ & $-0,186$ & 0,072 & $-0,159$ & 0,054 \\
\hline$\phi_{1,2}$ & $\{-0,2\}$ & $-0,167$ & 0,081 & $-0,140$ & 0,057 \\
\hline$\theta_{1,1}^{(\boldsymbol{\delta})}$ & $\{1,0\}$ & 1,007 & 0,086 & 1,043 & 0,067 \\
\hline$\theta_{2,2}^{(\boldsymbol{\delta})}$ & $\{1,0\}$ & 0,946 & 0,093 & 0,971 & 0,076 \\
\hline$\theta_{3,3}^{(\boldsymbol{\delta})}$ & $\{1,0\}$ & 0,928 & 0,132 & 0,934 & 0,097 \\
\hline$\theta_{4,4}^{(\boldsymbol{\delta})}$ & $\{1,0\}$ & 1,002 & 0,190 & 0,999 & 0,134 \\
\hline$\theta_{5,5}^{(\boldsymbol{\delta})}$ & $\{1,0\}$ & 1,230 & 0,122 & 1,088 & 0,083 \\
\hline$\theta_{6,6}^{(\widetilde{\delta})}$ & $\{1,0\}$ & 0,761 & 0,513 & 0,677 & 0,389 \\
\hline$\theta_{7.7}^{(\boldsymbol{\delta})}$ & $\{1,0\}$ & 1,075 & 0,088 & 1,036 & 0,063 \\
\hline$\theta_{8,8}^{(\boldsymbol{\delta})}$ & $\{1,0\}$ & 1,028 & 0,080 & 1,074 & 0,066 \\
\hline
\end{tabular}


Tabela 5.5: Estatisticas dos mil valores obtidos via simulação para os indicadores clássicos e robustos do MEE descrito pela Figura 5.1, por tamanho de amostra e com fração de 5\% de contaminação proveniente da lei Exponencial de média unitária.

\begin{tabular}{|c|c|c|c|c|c|c|c|c|c|}
\hline \multirow[b]{2}{*}{ Índice } & \multirow{3}{*}{$\begin{array}{c}\text { Referência } \\
{[0 ; 0,08[}\end{array}$} & \multicolumn{4}{|c|}{$n=100$} & \multicolumn{4}{|c|}{$n=200$} \\
\hline & & $\hat{\mu}$ & $\hat{\sigma}$ & $\hat{\pi}$ & $\hat{\sigma}_{\hat{\pi}}$ & $\hat{\mu}$ & $\hat{\sigma}$ & $\hat{\pi}$ & $\hat{\sigma}_{\hat{\pi}}$ \\
\hline SRMR & & 0,084 & 0,014 & 0,590 & 0,492 & 0,059 & 0,011 & 0,031 & 0,173 \\
\hline RMSEA & {$[0 ; 0,08[$} & 0,072 & 0,031 & 0,427 & 0,495 & 0,046 & 0,023 & 0,050 & 0,218 \\
\hline NFI & ] $0,90 ; 1]$ & 0,803 & 0,048 & 1,000 & 0,000 & 0,894 & 0,030 & 0,988 & 0,109 \\
\hline IFI & ] $0,90 ; 1]$ & 0,918 & 0,056 & 0,712 & 0,453 & 0,962 & 0,032 & 0,328 & 0,470 \\
\hline NNFI & ] $0,90 ; 1]$ & 0,871 & 0,088 & 0,83 & 0,376 & 0,942 & 0,049 & 0,543 & 0,498 \\
\hline CFI & ] $0,90 ; 1]$ & 0,911 & 0,057 & 0,737 & 0,440 & 0,959 & 0,031 & 0,351 & 0,478 \\
\hline RNI & ] $0,90 ; 1]$ & 0,912 & 0,060 & 0,737 & 0,440 & 0,961 & 0,033 & 0,351 & 0,478 \\
\hline $\mathrm{GFI}_{\mathrm{MV}}$ & ] $0,90 ; 1]$ & 0,929 & 0,017 & 0,889 & 0,314 & 0,965 & 0,010 & 0,066 & 0,248 \\
\hline $\mathrm{GFI}_{\mathrm{MV}}^{\mathrm{GKO}}$ & ] $0,90 ; 1]$ & 0,909 & 0,051 & 0,807 & 0,395 & 0,950 & 0,025 & 0,399 & 0,490 \\
\hline $\mathrm{GFI}_{\mathrm{MV}}^{\mathrm{EVM}}$ & ] $0,90 ; 1]$ & 0,933 & 0,032 & 0,668 & 0,471 & 0,974 & 0,011 & 0,027 & 0,162 \\
\hline $\mathrm{GFI}_{\mathrm{MV}}^{\mathrm{CDM}}$ & ] $0,90 ; 1]$ & 0,931 & 0,029 & 0,734 & 0,442 & 0,977 & 0,009 & 0,015 & 0,122 \\
\hline $\mathrm{GFI}_{\mathrm{MV}}^{\mathrm{S}}$ & ] $0,90 ; 1]$ & 0,965 & 0,015 & 0,141 & 0,348 & 0,986 & 0,005 & 0,000 & 0,000 \\
\hline $\mathrm{GFI}_{\mathrm{MV}}^{\mathrm{MM}}$ & ] $0,90 ; 1]$ & 0,970 & 0,014 & 0,086 & 0,281 & 0,988 & 0,004 & 0,000 & 0,000 \\
\hline $\mathrm{AGFI}_{\mathrm{MV}}$ & ]0,90;1] & 0,866 & 0,033 & 0,999 & 0,032 & 0,934 & 0,018 & 0,809 & 0,393 \\
\hline $\mathrm{AGFI}_{\mathrm{MV}}^{\mathrm{GKO}}$ & ] $0,90 ; 1]$ & 0,828 & 0,096 & 0,987 & 0,113 & 0,905 & 0,047 & 0,879 & 0,326 \\
\hline $\mathrm{AGFI}_{\mathrm{MV}}^{\mathrm{EVM}}$ & ] $0,90 ; 1]$ & 0,874 & 0,061 & 0,982 & 0,133 & 0,950 & 0,021 & 0,419 & 0,494 \\
\hline $\mathrm{AGFI}_{\mathrm{MV}}^{\mathrm{CDM}}$ & ] $0,90 ; 1]$ & 0,870 & 0,054 & 0,985 & 0,122 & 0,956 & 0,018 & 0,330 & 0,470 \\
\hline $\mathrm{AGFI}_{\mathrm{MV}}^{\mathrm{S}}$ & ] $0,90 ; 1]$ & 0,934 & 0,029 & 0,674 & 0,469 & 0,973 & 0,009 & 0,019 & 0,137 \\
\hline \multirow[t]{2}{*}{$\mathrm{AGFI}_{\mathrm{MV}}^{\mathrm{MM}}$} & ] $0,90 ; 1]$ & 0,942 & 0,026 & 0,545 & 0,498 & 0,977 & 0,008 & 0,008 & 0,089 \\
\hline & & \multicolumn{4}{|c|}{$n=300$} & \multicolumn{4}{|c|}{$n=500$} \\
\hline Índice & Referência & $\hat{\mu}$ & $\hat{\sigma}$ & $\hat{\pi}$ & $\hat{\sigma}_{\hat{\pi}}$ & $\hat{\mu}$ & $\hat{\sigma}$ & $\hat{\pi}$ & $\hat{\sigma}_{\hat{\pi}}$ \\
\hline SRMR & {$[0 ; 0,08[$} & 0,062 & 0,009 & 0,029 & 0,168 & 0,057 & 0,008 & 0,004 & 0,063 \\
\hline RMSEA & {$[0 ; 0,08[$} & 0,062 & 0,015 & 0,083 & 0,276 & 0,060 & 0,010 & 0,028 & 0,165 \\
\hline NFI & ] $0,90 ; 1]$ & 0,893 & 0,024 & 0,994 & 0,077 & 0,918 & 0,018 & 0,976 & 0,153 \\
\hline IFI & ] $0,90 ; 1]$ & 0,939 & 0,025 & 0,663 & 0,473 & 0,945 & 0,018 & 0,597 & 0,491 \\
\hline NNFI & ] $0,90 ; 1]$ & 0,908 & 0,037 & 0,870 & 0,336 & 0,918 & 0,027 & 0,901 & 0,299 \\
\hline CFI & ] $0,90 ; 1]$ & 0,938 & 0,025 & 0,688 & 0,464 & 0,944 & 0,018 & 0,614 & 0,487 \\
\hline RNI & ] $0,90 ; 1]$ & 0,938 & 0,025 & 0,688 & 0,464 & 0,944 & 0,018 & 0,614 & 0,487 \\
\hline $\mathrm{GFI}_{\mathrm{MV}}$ & ] $0,90 ; 1]$ & 0,965 & 0,008 & 0,036 & 0,186 & 0,972 & 0,006 & 0,001 & 0,032 \\
\hline $\mathrm{GFI}_{\mathrm{MV}}^{\mathrm{GKO}}$ & ] $0,90 ; 1]$ & 0,955 & 0,018 & 0,307 & 0,461 & 0,959 & 0,014 & 0,224 & 0,417 \\
\hline $\mathrm{GFI}_{\mathrm{MV}}^{\mathrm{EVM}}$ & ] $0,90 ; 1]$ & 0,982 & 0,007 & 0,001 & 0,032 & 0,987 & 0,005 & 0,000 & 0,000 \\
\hline $\mathrm{GFI}_{\mathrm{MV}}^{\mathrm{CDM}}$ & ] $0,90 ; 1]$ & 0,984 & 0,005 & 0,000 & 0,000 & 0,989 & 0,003 & 0,000 & 0,000 \\
\hline $\mathrm{GFI}_{\mathrm{MV}}^{\mathrm{S}}$ & ] $0,90 ; 1]$ & 0,988 & 0,004 & 0,000 & 0,000 & 0,991 & 0,003 & 0,000 & 0,000 \\
\hline $\mathrm{GFI}_{\mathrm{MV}}^{\mathrm{MM}}$ & ] $0,90 ; 1]$ & 0,989 & 0,004 & 0,000 & 0,000 & 0,992 & 0,003 & 0,000 & 0,000 \\
\hline $\mathrm{AGFI}_{\mathrm{MV}}$ & ] $0,90 ; 1]$ & 0,934 & 0,015 & 0,854 & 0,353 & 0,947 & 0,012 & 0,590 & 0,492 \\
\hline $\mathrm{AGFI}_{\mathrm{MV}}^{\mathrm{GKO}}$ & ] $0,90 ; 1]$ & 0,915 & 0,034 & 0,891 & 0,312 & 0,923 & 0,026 & 0,886 & 0,318 \\
\hline $\mathrm{AGFI}_{\mathrm{MV}}^{\mathrm{EVM}}$ & ] $0,90 ; 1]$ & 0,965 & 0,013 & 0,127 & 0,333 & 0,976 & 0,009 & 0,014 & 0,118 \\
\hline $\mathrm{AGFI}_{\mathrm{MV}}^{\mathrm{CDM}}$ & ] $0,90 ; 1]$ & 0,970 & 0,010 & 0,042 & 0,201 & 0,980 & 0,007 & 0,000 & 0,000 \\
\hline $\mathrm{AGFI}_{\mathrm{MV}}^{\mathrm{S}}$ & ] $0,90 ; 1]$ & 0,977 & 0,007 & 0,003 & 0,055 & 0,983 & 0,005 & 0,000 & 0,000 \\
\hline $\mathrm{AGFI}_{\mathrm{MV}}^{\mathrm{MM}}$ & ] $0,90 ; 1]$ & 0,980 & 0,007 & 0,001 & 0,032 & 0,984 & 0,005 & 0,000 & 0,000 \\
\hline
\end{tabular}


Tabela 5.6: Estatísticas dos mil valores obtidos via simulação para as estimativas dos parâmetros do MEE descrito pela Figura 5.1, por tamanho de amostra e com fração de $25 \%$ de contaminação proveniente da lei Exponencial de média unitária.

\begin{tabular}{|c|c|c|c|c|c|}
\hline \multirow{3}{*}{$\begin{array}{c}\text { Parâmetro } \\
\lambda_{1,1}^{(\boldsymbol{x})}\end{array}$} & \multirow{3}{*}{$\begin{array}{c}\text { Referência } \\
\qquad\{0,3\}\end{array}$} & \multicolumn{2}{|c|}{$n=100$} & \multicolumn{2}{|c|}{$n=200$} \\
\hline & & $\hat{\mu}_{\hat{\theta}}$ & $\hat{\sigma}_{\hat{\theta}}$ & $\hat{\mu}_{\hat{\theta}}$ & $\hat{\sigma}_{\hat{\theta}}$ \\
\hline & & 0,819 & 0,107 & 0,596 & 0,063 \\
\hline$\lambda_{2,1}^{(\boldsymbol{x})}$ & $\{0,6\}$ & 0,976 & 0,104 & 0,873 & 0,069 \\
\hline$\lambda_{3,1}^{(\boldsymbol{x})}$ & $\{0,9\}$ & 1,056 & 0,131 & 1,159 & 0,083 \\
\hline$\lambda_{4,1}^{(\boldsymbol{x})}$ & $\{1,2\}$ & 1,250 & 0,156 & 1,407 & 0,100 \\
\hline$\lambda_{5,2}^{(\boldsymbol{x})}$ & $\{-0,7\}$ & $-0,002$ & 0,550 & 0,058 & 0,857 \\
\hline$\lambda_{6,2}^{(\boldsymbol{x})}$ & $\{1,8\}$ & 0,752 & 0,594 & 0,452 & 0,614 \\
\hline$\lambda_{7,2}^{(\boldsymbol{x})}$ & $\{0,5\}$ & 0,678 & 0,500 & 0,436 & 0,513 \\
\hline$\lambda_{8,2}^{(\boldsymbol{x})}$ & $\{-0,2\}$ & 0,302 & 0,509 & 0,145 & 0,498 \\
\hline$\phi_{1,2}$ & $\{-0,2\}$ & 0,313 & 0,483 & 0,183 & 0,540 \\
\hline$\theta_{1,1}^{(\boldsymbol{\delta})}$ & $\{1,0\}$ & 0,944 & 0,180 & 0,797 & 0,100 \\
\hline$\theta_{2,2}^{(\hat{\boldsymbol{d}})}$ & $\{1,0\}$ & 0,721 & 0,169 & 0,740 & 0,105 \\
\hline$\theta_{33}^{(\boldsymbol{\delta})}$ & $\{1,0\}$ & 0,801 & 0,192 & 0,734 & 0,128 \\
\hline$\theta_{4.4}^{(\boldsymbol{\delta})}$ & $\{1,0\}$ & 1,027 & 0,241 & 0,871 & 0,168 \\
\hline$\theta_{5,5}^{(\boldsymbol{\delta})}$ & $\{1,0\}$ & 1,144 & 0,409 & 1,064 & 0,550 \\
\hline$\theta_{6,6}^{(\boldsymbol{\delta})}$ & $\{1,0\}$ & 3,311 & 0,779 & 3,732 & 0,586 \\
\hline$\theta_{7,7}^{(\boldsymbol{\delta})}$ & $\{1,0\}$ & 0,709 & 0,513 & 0,927 & 0,523 \\
\hline \multirow[t]{2}{*}{$\begin{array}{r}\theta_{8,8}^{(\boldsymbol{\delta})} \\
\end{array}$} & $\{1,0\}$ & 0,933 & 0,281 & 0,813 & 0,172 \\
\hline & & \multicolumn{2}{|c|}{$n=300$} & \multicolumn{2}{|c|}{$n=500$} \\
\hline \multirow{2}{*}{$\begin{array}{c}\text { Parâmetro } \\
\lambda_{1,1}^{(\boldsymbol{x})}\end{array}$} & \multirow{2}{*}{$\begin{array}{c}\text { Referência } \\
\qquad\{0,3\}\end{array}$} & $\hat{\mu}_{\hat{\theta}}$ & $\hat{\sigma}_{\hat{\theta}}$ & $\hat{\mu}_{\hat{\theta}}$ & $\hat{\sigma}_{\hat{\theta}}$ \\
\hline & & 0,699 & 0,057 & 0,634 & 0,043 \\
\hline$\lambda_{2,1}^{(\boldsymbol{x})}$ & $\{0,6\}$ & 0,897 & 0,060 & 0,869 & 0,046 \\
\hline$\lambda_{3,1}^{(\boldsymbol{x})}$ & $\{0,9\}$ & 1,214 & 0,068 & 1,088 & 0,052 \\
\hline$\lambda_{4,1}^{(\boldsymbol{x})}$ & $\{1,2\}$ & 1,206 & 0,079 & 1,342 & 0,063 \\
\hline$\lambda_{5,2}^{(x)}$ & $\{-0,7\}$ & 0,075 & 0,435 & 0,225 & 0,482 \\
\hline$\lambda_{6,2}^{(\boldsymbol{x})}$ & $\{1,8\}$ & 0,995 & 0,468 & 0,829 & 0,585 \\
\hline$\lambda_{7,2}^{(\boldsymbol{x})}$ & $\{0,5\}$ & 0,918 & 0,403 & 0,837 & 0,424 \\
\hline$\lambda_{8,2}^{(\boldsymbol{x})}$ & $\{-0,2\}$ & 0,218 & 0,267 & 0,353 & 0,327 \\
\hline$\phi_{1,2}$ & $\{-0,2\}$ & 0,299 & 0,298 & 0,329 & 0,279 \\
\hline$\theta_{1,1}^{(\boldsymbol{\delta})}$ & $\{1,0\}$ & 0,872 & 0,092 & 0,850 & 0,063 \\
\hline$\theta_{2,2}^{(\boldsymbol{\delta})}$ & $\{1,0\}$ & 0,747 & 0,093 & 0,745 & 0,071 \\
\hline$\theta_{3,3}^{(\boldsymbol{\delta})}$ & $\{1,0\}$ & 0,690 & 0,114 & 0,745 & 0,079 \\
\hline$\theta_{4,4}^{(\boldsymbol{\delta})}$ & $\{1,0\}$ & 1,054 & 0,124 & 0,885 & 0,103 \\
\hline$\theta_{5,5}^{(\boldsymbol{\delta})}$ & $\{1,0\}$ & 1,531 & 0,390 & 1,527 & 0,423 \\
\hline$\theta_{6,6}^{(\boldsymbol{\delta})}$ & $\{1,0\}$ & 3,308 & 0,546 & 3,509 & 0,625 \\
\hline$\theta_{7,7}^{(\boldsymbol{\delta})}$ & $\{1,0\}$ & 0,377 & 0,445 & 0,468 & 0,508 \\
\hline$\theta_{8,8}^{(\boldsymbol{\delta})}$ & $\{1,0\}$ & 0,951 & 0,148 & 1,018 & 0,227 \\
\hline
\end{tabular}


Tabela 5.7: Estatisticas dos mil valores obtidos via simulação para os indicadores clássicos e robustos do MEE descrito pela Figura 5.1, por tamanho de amostra e com fração de $25 \%$ de contaminação proveniente da lei Exponencial de média unitária.

\begin{tabular}{|c|c|c|c|c|c|c|c|c|c|}
\hline \multirow{3}{*}{$\begin{array}{l}\text { Îndice } \\
\text { SRMR }\end{array}$} & \multirow{3}{*}{$\begin{array}{c}\text { Referência } \\
{[0 ; 0,08[}\end{array}$} & \multicolumn{4}{|c|}{$n=100$} & \multicolumn{4}{|c|}{$n=200$} \\
\hline & & $\hat{\mu}$ & $\hat{\sigma}$ & $\hat{\pi}$ & $\hat{\sigma}_{\hat{\pi}}$ & $\hat{\mu}$ & $\hat{\sigma}$ & $\hat{\pi}$ & $\hat{\sigma}_{\hat{\pi}}$ \\
\hline & & 0,140 & 0,024 & $\overline{1,000}$ & 0,000 & 0,124 & 0,022 & 1,000 & 0,000 \\
\hline RMSEA & {$[0 ; 0,08[$} & 0,183 & 0,021 & 1,000 & 0,000 & 0,159 & 0,015 & 1,000 & 0,000 \\
\hline NFI & ] $0,90 ; 1]$ & 0,706 & 0,047 & 1,000 & 0,000 & 0,771 & 0,033 & 1,000 & 0,000 \\
\hline IFI & ] $0,90 ; 1]$ & 0,757 & 0,049 & 1,000 & 0,000 & 0,802 & 0,034 & 1,000 & 0,000 \\
\hline NNFI & ] $0,90 ; 1]$ & 0,630 & 0,075 & 1,000 & 0,000 & 0,702 & 0,050 & 1,000 & 0,000 \\
\hline CFI & ] $0,90 ; 1]$ & 0,749 & 0,051 & 1,000 & 0,000 & 0,798 & 0,034 & 1,000 & 0,000 \\
\hline RNI & ] $0,90 ; 1]$ & 0,749 & 0,051 & 1,000 & 0,000 & 0,798 & 0,034 & 1,000 & 0,000 \\
\hline $\mathrm{GFI}_{\mathrm{MV}}$ & ] $0,90 ; 1]$ & 0,831 & 0,025 & 1,000 & 0,000 & 0,878 & 0,017 & 1,000 & 0,000 \\
\hline $\mathrm{GFI}_{\mathrm{MV}}^{\mathrm{GKO}}$ & ] $0,90 ; 1]$ & 0,705 & 0,123 & 1,000 & 0,000 & 0,810 & 0,056 & 1,000 & 0,000 \\
\hline $\mathrm{GFI}_{\mathrm{MV}}^{\mathrm{EVM}}$ & ] $0,90 ; 1]$ & 0,834 & 0,068 & 0,999 & 0,032 & 0,883 & 0,041 & 0,995 & 0,071 \\
\hline $\mathrm{GFI}_{\mathrm{MV}}^{\mathrm{CDM}}$ & ] $0,90 ; 1]$ & 0,818 & 0,046 & 1,000 & 0,000 & 0,860 & 0,031 & 1,000 & 0,000 \\
\hline $\mathrm{GFI}_{\mathrm{MV}}^{\mathrm{S}}$ & ] $0,90 ; 1]$ & 0,871 & 0,044 & 0,993 & 0,083 & 0,905 & 0,022 & 0,997 & 0,055 \\
\hline $\mathrm{GFI}_{\mathrm{MV}}^{\mathrm{MM}}$ & ] $0,90 ; 1]$ & 0,876 & 0,038 & 0,992 & 0,089 & 0,906 & 0,020 & 0,997 & 0,055 \\
\hline $\mathrm{AGFI}_{\mathrm{MV}}$ & ] $0,90 ; 1]$ & 0,680 & 0,048 & 1,000 & 0,000 & 0,768 & 0,033 & 1,000 & 0,000 \\
\hline $\mathrm{AGFI}_{\mathrm{MV}}^{\mathrm{GKO}}$ & ] $0,90 ; 1]$ & 0,441 & 0,233 & 1,000 & 0,000 & 0,640 & 0,106 & 1,000 & 0,000 \\
\hline $\mathrm{AGFI}_{\mathrm{MV}}^{\mathrm{EVM}}$ & ] $0,90 ; 1]$ & 0,686 & 0,129 & 1,000 & 0,000 & 0,778 & 0,077 & 1,000 & 0,000 \\
\hline $\mathrm{AGFI}_{\mathrm{MV}}^{\mathrm{CDM}}$ & ] $0,90 ; 1]$ & 0,655 & 0,086 & 1,000 & 0,000 & 0,735 & 0,060 & 1,000 & 0,000 \\
\hline $\mathrm{AGFI}_{\mathrm{MV}}^{\mathrm{S}}$ & ] $0,90 ; 1]$ & 0,756 & 0,083 & 1,000 & 0,000 & 0,820 & 0,041 & 1,000 & 0,000 \\
\hline \multirow[t]{2}{*}{$\mathrm{AGFI}_{\mathrm{MV}}^{\mathrm{MM}}$} & ] $0,90 ; 1]$ & 0,765 & 0,073 & 1,000 & 0,000 & 0,823 & 0,037 & 1,000 & 0,000 \\
\hline & & \multicolumn{4}{|c|}{$n=300$} & \multicolumn{4}{|c|}{$n=500$} \\
\hline Índice & Referência & $\hat{\mu}$ & $\hat{\sigma}$ & $\hat{\pi}$ & $\hat{\sigma}_{\hat{\pi}}$ & $\hat{\mu}$ & $\hat{\sigma}$ & $\hat{\pi}$ & $\hat{\sigma}_{\hat{\pi}}$ \\
\hline SRMR & {$[0 ; 0,08[$} & 0,145 & 0,020 & 1,000 & 0,000 & 0,148 & 0,025 & 1,000 & 0,000 \\
\hline RMSEA & {$[0 ; 0,08[$} & 0,174 & 0,012 & 1,000 & 0,000 & 0,179 & 0,010 & 1,000 & 0,000 \\
\hline NFI & ] $0,90 ; 1]$ & 0,744 & 0,029 & 1,000 & 0,000 & 0,740 & 0,027 & 1,000 & 0,000 \\
\hline IFI & ] $0,90 ; 1]$ & 0,763 & 0,030 & 1,000 & 0,000 & 0,751 & 0,027 & 1,000 & 0,000 \\
\hline NNFI & ] $0,90 ; 1]$ & 0,647 & 0,045 & 1,000 & 0,000 & 0,631 & 0,040 & 1,000 & 0,000 \\
\hline CFI & ] $0,90 ; 1]$ & 0,760 & 0,030 & 1,000 & 0,000 & 0,750 & 0,027 & 1,000 & 0,000 \\
\hline RNI & ] $0,90 ; 1]$ & 0,760 & 0,030 & 1,000 & 0,000 & 0,750 & 0,027 & 1,000 & 0,000 \\
\hline $\mathrm{GFI}_{\mathrm{MV}}$ & ]0,90;1] & 0,865 & 0,014 & 1,000 & 0,000 & 0,862 & 0,015 & 1,000 & 0,000 \\
\hline $\mathrm{GFI}_{\mathrm{MV}}^{\mathrm{GKO}}$ & ] $0,90 ; 1]$ & 0,799 & 0,049 & 1,000 & 0,000 & 0,819 & 0,032 & 1,000 & 0,000 \\
\hline $\mathrm{GFI}_{\mathrm{MV}}^{\mathrm{EVM}}$ & ] $0,90 ; 1]$ & 0,873 & 0,038 & 1,000 & 0,000 & 0,875 & 0,032 & 1,000 & 0,000 \\
\hline $\mathrm{GFI}_{\mathrm{MV}}^{\mathrm{CDM}}$ & ] $0,90 ; 1]$ & 0,855 & 0,027 & 1,000 & 0,000 & 0,839 & 0,022 & 1,000 & 0,000 \\
\hline $\mathrm{GFI}_{\mathrm{MV}}^{\mathrm{S}}$ & ] $0,90 ; 1]$ & 0,895 & 0,021 & 1,000 & 0,000 & 0,899 & 0,015 & 1,000 & 0,000 \\
\hline $\mathrm{GFI}_{\mathrm{MV}}^{\mathrm{MM}}$ & ] $0,90 ; 1]$ & 0,898 & 0,019 & 0,999 & 0,032 & 0,899 & 0,014 & 1,000 & 0,000 \\
\hline $\mathrm{AGFI}_{\mathrm{MV}}$ & ] $0,90 ; 1]$ & 0,744 & 0,027 & 1,000 & 0,000 & 0,738 & 0,028 & 1,000 & 0,000 \\
\hline $\mathrm{AGFI}_{\mathrm{MV}}^{\mathrm{GKO}}$ & ] $0,90 ; 1]$ & 0,618 & 0,093 & 1,000 & 0,000 & 0,657 & 0,061 & 1,000 & 0,000 \\
\hline $\mathrm{AGFI}_{\mathrm{MV}}^{\mathrm{EVM}}$ & ] $0,90 ; 1]$ & 0,759 & 0,072 & 1,000 & 0,000 & 0,764 & 0,061 & 1,000 & 0,000 \\
\hline $\mathrm{AGFI}_{\mathrm{MV}}^{\mathrm{CDM}}$ & ] $0,90 ; 1]$ & 0,725 & 0,052 & 1,000 & 0,000 & 0,695 & 0,042 & 1,000 & 0,000 \\
\hline $\mathrm{AGFI}_{\mathrm{MV}}^{\mathrm{S}}$ & ] $0,90 ; 1]$ & 0,800 & 0,040 & 1,000 & 0,000 & 0,808 & 0,028 & 1,000 & 0,000 \\
\hline $\mathrm{AGFI}_{\mathrm{MV}}^{\mathrm{MM}}$ & ] $0,90 ; 1]$ & 0,807 & 0,036 & 1,000 & 0,000 & 0,808 & 0,026 & 1,000 & 0,000 \\
\hline
\end{tabular}


Tabela 5.8: Estatísticas dos mil valores obtidos via simulação para as estimativas dos parâmetros do MEE descrito pela Figura 5.1, por tamanho de amostra e com fração de 5\% de contaminação proveniente da lei t-Student com cinco graus de liberdade.

\begin{tabular}{|c|c|c|c|c|c|}
\hline \multirow{3}{*}{$\begin{array}{c}\text { Parâmetro } \\
\lambda_{1.1}^{(\boldsymbol{x})}\end{array}$} & \multirow{3}{*}{$\begin{array}{c}\text { Referência } \\
\quad\{0,3\}\end{array}$} & \multicolumn{2}{|c|}{$n=100$} & \multicolumn{2}{|c|}{$n=200$} \\
\hline & & $\hat{\mu}_{\hat{\theta}}$ & $\hat{\sigma}_{\hat{\theta}}$ & $\hat{\mu}_{\hat{\theta}}$ & $\hat{\sigma}_{\hat{\theta}}$ \\
\hline & & 0,329 & 0,120 & 0,351 & 0,084 \\
\hline$\lambda_{2,1}^{(\boldsymbol{x})}$ & $\{0,6\}$ & 0,604 & 0,124 & 0,622 & 0,087 \\
\hline$\lambda_{3,1}^{(\boldsymbol{x})}$ & $\{0,9\}$ & 0,914 & 0,152 & 0,950 & 0,111 \\
\hline$\lambda_{4,1}^{(\boldsymbol{x})}$ & $\{1,2\}$ & 1,221 & 0,187 & 1,177 & 0,124 \\
\hline$\lambda_{5,2}^{(\boldsymbol{x})}$ & $\{-0,7\}$ & $-0,555$ & 0,497 & $-0,638$ & 0,303 \\
\hline$\lambda_{6,2}^{(\boldsymbol{x})}$ & $\{1,8\}$ & 1,418 & 1,002 & 1,730 & 0,670 \\
\hline$\lambda_{7,2}^{(\boldsymbol{x})}$ & $\{0,5\}$ & 0,431 & 0,337 & 0,484 & 0,215 \\
\hline$\lambda_{8,2}^{(\boldsymbol{x})}$ & $\{-0,2\}$ & $-0,161$ & 0,173 & $-0,164$ & 0,106 \\
\hline$\phi_{1,2}$ & $\{-0,2\}$ & $-0,154$ & 0,180 & $-0,162$ & 0,116 \\
\hline$\theta_{1,1}^{(\boldsymbol{\delta})}$ & $\{1,0\}$ & 0,958 & 0,142 & 0,974 & 0,104 \\
\hline$\theta_{2,2}^{(\boldsymbol{\delta})}$ & $\{1,0\}$ & 0,938 & 0,166 & 0,941 & 0,119 \\
\hline$\theta_{3,3}^{(\boldsymbol{\delta})}$ & $\{1,0\}$ & 0,923 & 0,222 & 0,973 & 0,167 \\
\hline$\theta_{4,4}^{(\boldsymbol{\delta})}$ & $\{1,0\}$ & 0,967 & 0,342 & 1,010 & 0,232 \\
\hline$\theta_{5,5}^{(\boldsymbol{\delta})}$ & $\{1,0\}$ & 0,937 & 0,187 & 0,988 & 0,128 \\
\hline$\theta_{6,6}^{(\boldsymbol{\delta})}$ & $\{1,0\}$ & 1,345 & 0,651 & 0,956 & 0,517 \\
\hline$\theta_{7,7}^{(\boldsymbol{\delta})}$ & $\{1,0\}$ & 0,915 & 0,156 & 0,993 & 0,104 \\
\hline \multirow[t]{2}{*}{$\theta_{8,8}^{(\boldsymbol{\delta})}$} & $\{1,0\}$ & 1,009 & 0,145 & 1,089 & 0,101 \\
\hline & & \multicolumn{2}{|c|}{$n=300$} & \multicolumn{2}{|c|}{$n=500$} \\
\hline \multirow{2}{*}{$\begin{array}{c}\text { Parâmetro } \\
\lambda_{1,1}^{(\boldsymbol{x})}\end{array}$} & \multirow{2}{*}{$\begin{array}{c}\text { Referência } \\
\quad\{0,3\}\end{array}$} & $\hat{\mu}_{\hat{\theta}}$ & $\hat{\sigma}_{\hat{\theta}}$ & $\hat{\mu}_{\hat{\theta}}$ & $\hat{\sigma}_{\hat{\theta}}$ \\
\hline & & 0,349 & 0,067 & 0,356 & 0,051 \\
\hline$\lambda_{2,1}^{(\boldsymbol{x})}$ & $\{0,6\}$ & 0,643 & 0,073 & 0,628 & 0,055 \\
\hline$\lambda_{3,1}^{(x)}$ & $\{0,9\}$ & 0,906 & 0,080 & 0,958 & 0,067 \\
\hline$\lambda_{4,1}^{(\boldsymbol{x})}$ & $\{1,2\}$ & 1,259 & 0,102 & 1,246 & 0,079 \\
\hline$\lambda_{5,2}^{(\boldsymbol{x})}$ & $\{-0,7\}$ & $-0,655$ & 0,179 & $-0,665$ & 0,068 \\
\hline$\lambda_{6,2}^{(\boldsymbol{x})}$ & $\{1,8\}$ & 1,827 & 0,408 & 1,878 & 0,132 \\
\hline$\lambda_{7,2}^{(\boldsymbol{x})}$ & $\{0,5\}$ & 0,514 & 0,135 & 0,514 & 0,052 \\
\hline$\lambda_{8,2}^{(\boldsymbol{x})}$ & $\{-0,2\}$ & $-0,165$ & 0,080 & $-0,176$ & 0,056 \\
\hline$\phi_{1,2}$ & $\{-0,2\}$ & $-0,160$ & 0,085 & $-0,159$ & 0,059 \\
\hline$\theta_{1,1}^{(\boldsymbol{\delta})}$ & $\{1,0\}$ & 0,980 & 0,084 & 0,981 & 0,064 \\
\hline$\theta_{2,2}^{(\boldsymbol{\delta})}$ & $\{1,0\}$ & 0,965 & 0,095 & 0,958 & 0,069 \\
\hline$\theta_{3,3}^{(\tilde{\boldsymbol{\delta}})}$ & $\{1,0\}$ & 0,970 & 0,122 & 0,959 & 0,099 \\
\hline$\theta_{4,4}^{(\boldsymbol{\delta})}$ & $\{1,0\}$ & 0,934 & 0,191 & 0,944 & 0,135 \\
\hline$\theta_{5,5}^{(\boldsymbol{\delta})}$ & $\{1,0\}$ & 1,043 & 0,108 & 1,113 & 0,084 \\
\hline$\theta_{6,6}^{(\boldsymbol{\delta})}$ & $\{1,0\}$ & 0,927 & 0,486 & 0,825 & 0,415 \\
\hline$\theta_{7,7}^{(\boldsymbol{\delta})}$ & $\{1,0\}$ & 1,011 & 0,085 & 1,005 & 0,065 \\
\hline$\theta_{8,8}^{(\boldsymbol{\delta})}$ & $\{1,0\}$ & 1,108 & 0,081 & 1,052 & 0,063 \\
\hline
\end{tabular}


Tabela 5.9: Estatisticas dos mil valores obtidos via simulação para os indicadores clássicos e robustos do MEE descrito pela Figura 5.1, por tamanho de amostra e com fração de 5\% de contaminação proveniente da lei t-Student com cinco graus de liberdade.

\begin{tabular}{|c|c|c|c|c|c|c|c|c|c|}
\hline \multirow[b]{2}{*}{ Índice } & \multirow{3}{*}{$\begin{array}{c}\text { Referência } \\
{[0 ; 0,08[}\end{array}$} & \multicolumn{4}{|c|}{$n=100$} & \multicolumn{4}{|c|}{$n=200$} \\
\hline & & $\hat{\mu}$ & $\hat{\sigma}$ & $\hat{\pi}$ & $\hat{\sigma}_{\hat{\pi}}$ & $\hat{\mu}$ & $\hat{\sigma}$ & $\hat{\pi}$ & $\hat{\sigma}_{\hat{\pi}}$ \\
\hline SRMR & & 0,063 & 0,012 & 0,082 & 0,275 & 0,053 & 0,010 & 0,007 & 0,083 \\
\hline RMSEA & {$[0 ; 0,08[$} & 0,026 & 0,030 & 0,051 & 0,220 & 0,034 & 0,024 & 0,018 & 0,133 \\
\hline NFI & ] $0,90 ; 1]$ & 0,857 & 0,045 & 0,998 & 0,045 & 0,904 & 0,028 & 0,971 & 0,168 \\
\hline IFI & ] $0,90 ; 1]$ & 0,995 & 0,052 & 0,186 & 0,389 & 0,976 & 0,030 & 0,184 & 0,388 \\
\hline NNFI & ] $0,90 ; 1]$ & 0,992 & 0,083 & 0,274 & 0,446 & 0,963 & 0,046 & 0,348 & 0,477 \\
\hline CFI & ] $0,90 ; 1]$ & 0,975 & 0,037 & 0,205 & 0,404 & 0,972 & 0,027 & 0,201 & 0,401 \\
\hline RNI & ]0,90;1] & 0,994 & 0,057 & 0,205 & 0,404 & 0,975 & 0,031 & 0,201 & 0,401 \\
\hline $\mathrm{GFI}_{\mathrm{MV}}$ & ] $0,90 ; 1]$ & 0,954 & 0,014 & 0,345 & 0,476 & 0,970 & 0,009 & 0,029 & 0,168 \\
\hline $\mathrm{GFI}_{\mathrm{MV}}^{\mathrm{GKO}}$ & ] $0,90 ; 1]$ & 0,935 & 0,042 & 0,573 & 0,495 & 0,955 & 0,024 & 0,312 & 0,464 \\
\hline $\mathrm{GFI}_{\mathrm{MV}}^{\mathrm{EVM}}$ & ] $0,90 ; 1]$ & 0,940 & 0,028 & 0,567 & 0,496 & 0,973 & 0,013 & 0,040 & 0,196 \\
\hline $\mathrm{GFI}_{\mathrm{MV}}^{\mathrm{CDM}}$ & ] $0,90 ; 1]$ & 0,936 & 0,026 & 0,694 & 0,461 & 0,976 & 0,010 & 0,023 & 0,150 \\
\hline $\mathrm{GFI}_{\mathrm{MV}}^{\mathrm{S}}$ & ] $0,90 ; 1]$ & 0,976 & 0,010 & 0,022 & 0,147 & 0,986 & 0,005 & 0,000 & 0,000 \\
\hline $\mathrm{GFI}_{\mathrm{MV}}^{\mathrm{MM}}$ & ] $0,90 ; 1]$ & 0,981 & 0,008 & 0,001 & 0,032 & 0,988 & 0,004 & 0,000 & 0,000 \\
\hline $\mathrm{AGFI}_{\mathrm{MV}}$ & ]0,90;1] & 0,913 & 0,026 & 0,948 & 0,222 & 0,943 & 0,017 & 0,635 & 0,482 \\
\hline $\mathrm{AGFI}_{\mathrm{MV}}^{\mathrm{GKO}}$ & ] $0,90 ; 1]$ & 0,876 & 0,079 & 0,916 & 0,278 & 0,915 & 0,045 & 0,813 & 0,390 \\
\hline $\mathrm{AGFI}_{\mathrm{MV}}^{\mathrm{EVM}}$ & ] $0,90 ; 1]$ & 0,887 & 0,053 & 0,944 & 0,230 & 0,950 & 0,025 & 0,401 & 0,490 \\
\hline $\mathrm{AGFI}_{\mathrm{MV}}^{\mathrm{CDM}}$ & ] $0,90 ; 1]$ & 0,878 & 0,049 & 0,982 & 0,133 & 0,955 & 0,019 & 0,324 & 0,468 \\
\hline $\mathrm{AGFI}_{\mathrm{MV}}^{\mathrm{S}}$ & ] $0,90 ; 1]$ & 0,955 & 0,019 & 0,319 & 0,466 & 0,973 & 0,010 & 0,027 & 0,162 \\
\hline \multirow[t]{2}{*}{$\mathrm{AGFI}_{\mathrm{MV}}^{\mathrm{MM}}$} & ] $0,90 ; 1]$ & 0,964 & 0,015 & 0,157 & 0,364 & 0,977 & 0,008 & 0,009 & 0,094 \\
\hline & & \multicolumn{4}{|c|}{$n=300$} & \multicolumn{4}{|c|}{$n=500$} \\
\hline Índice & Referência & $\hat{\mu}$ & $\hat{\sigma}$ & $\hat{\pi}$ & $\hat{\sigma}_{\hat{\pi}}$ & $\hat{\mu}$ & $\hat{\sigma}$ & $\hat{\pi}$ & $\hat{\sigma}_{\hat{\pi}}$ \\
\hline SRMR & {$[0 ; 0,08[$} & 0,052 & 0,009 & 0,004 & 0,063 & 0,046 & 0,008 & 0,000 & 0,000 \\
\hline RMSEA & {$[0 ; 0,08[$} & 0,043 & 0,018 & 0,006 & 0,077 & 0,044 & 0,013 & 0,000 & 0,000 \\
\hline NFI & ]0,90;1] & 0,919 & 0,022 & 0,937 & 0,243 & 0,938 & 0,016 & 0,769 & 0,422 \\
\hline IFI & ] $0,90 ; 1]$ & 0,967 & 0,023 & 0,220 & 0,414 & 0,968 & 0,016 & 0,149 & 0,356 \\
\hline NNFI & ] $0,90 ; 1]$ & 0,950 & 0,034 & 0,462 & 0,499 & 0,952 & 0,024 & 0,442 & 0,497 \\
\hline CFI & ] $0,90 ; 1]$ & 0,966 & 0,023 & 0,237 & 0,425 & 0,967 & 0,016 & 0,156 & 0,363 \\
\hline RNI & ] $0,90 ; 1]$ & 0,966 & 0,023 & 0,237 & 0,425 & 0,967 & 0,016 & 0,156 & 0,363 \\
\hline $\mathrm{GFI}_{\mathrm{MV}}$ & ] $0,90 ; 1]$ & 0,974 & 0,007 & 0,003 & 0,055 & 0,980 & 0,005 & 0,000 & 0,000 \\
\hline $\mathrm{GFI}_{\mathrm{MV}}^{\mathrm{GKO}}$ & ] $0,90 ; 1]$ & 0,958 & 0,018 & 0,271 & 0,445 & 0,965 & 0,012 & 0,112 & 0,316 \\
\hline $\mathrm{GFI}_{\mathrm{MV}}^{\mathrm{EVM}}$ & ] $0,90 ; 1]$ & 0,983 & 0,007 & 0,002 & 0,045 & 0,989 & 0,004 & 0,000 & 0,000 \\
\hline $\mathrm{GFI}_{\mathrm{MV}}^{\mathrm{CDM}}$ & ] $0,90 ; 1]$ & 0,986 & 0,006 & 0,000 & 0,000 & 0,991 & 0,003 & 0,000 & 0,000 \\
\hline $\mathrm{GFI}_{\mathrm{MV}}^{\mathrm{S}}$ & ] $0,90 ; 1]$ & 0,990 & 0,004 & 0,000 & 0,000 & 0,993 & 0,002 & 0,000 & 0,000 \\
\hline $\mathrm{GFI}_{\mathrm{MV}}^{\mathrm{MM}}$ & ] $0,90 ; 1]$ & 0,991 & 0,003 & 0,000 & 0,000 & 0,994 & 0,002 & 0,000 & 0,000 \\
\hline $\mathrm{AGFI}_{\mathrm{MV}}$ & ] $0,90 ; 1]$ & 0,951 & 0,013 & 0,428 & 0,495 & 0,963 & 0,010 & 0,113 & 0,317 \\
\hline $\mathrm{AGFI}_{\mathrm{MV}}^{\mathrm{GKO}}$ & ] $0,90 ; 1]$ & 0,921 & 0,034 & 0,810 & 0,392 & 0,933 & 0,023 & 0,754 & 0,431 \\
\hline $\mathrm{AGFI}_{\mathrm{MV}}^{\mathrm{EVM}}$ & ] $0,90 ; 1]$ & 0,968 & 0,014 & 0,084 & 0,278 & 0,979 & 0,008 & 0,012 & 0,109 \\
\hline $\mathrm{AGFI}_{\mathrm{MV}}^{\mathrm{CDM}}$ & ] $0,90 ; 1]$ & 0,973 & 0,011 & 0,032 & 0,176 & 0,983 & 0,006 & 0,000 & 0,000 \\
\hline $\mathrm{AGFI}_{\mathrm{MV}}^{\mathrm{S}}$ & ] $0,90 ; 1]$ & 0,981 & 0,007 & 0,001 & 0,032 & 0,986 & 0,004 & 0,000 & 0,000 \\
\hline $\mathrm{AGFI}_{\mathrm{MV}}^{\mathrm{MM}}$ & ] $0,90 ; 1]$ & 0,983 & 0,006 & 0,000 & 0,000 & 0,988 & 0,004 & 0,000 & 0,000 \\
\hline
\end{tabular}


Tabela 5.10: Estatísticas dos mil valores obtidos via simulação para as estimativas dos parâmetros do MEE descrito pela Figura 5.1, por tamanho de amostra e com fração de $25 \%$ de contaminação proveniente da lei t-Student com cinco graus de liberdade.

\begin{tabular}{|c|c|c|c|c|c|}
\hline \multirow{3}{*}{$\begin{array}{c}\text { Parâmetro } \\
\lambda_{1,1}^{(\boldsymbol{x})}\end{array}$} & \multirow{3}{*}{$\begin{array}{c}\text { Referência } \\
\quad\{0,3\}\end{array}$} & \multicolumn{2}{|c|}{$n=100$} & \multicolumn{2}{|c|}{$n=200$} \\
\hline & & $\hat{\mu}_{\hat{\theta}}$ & $\hat{\sigma}_{\hat{\theta}}$ & $\hat{\mu}_{\hat{\theta}}$ & $\hat{\sigma}_{\hat{\theta}}$ \\
\hline & & 0,560 & 0,097 & 0,664 & 0,067 \\
\hline$\lambda_{2,1}^{(\boldsymbol{x})}$ & $\{0,6\}$ & 0,755 & 0,097 & 0,972 & 0,073 \\
\hline$\lambda_{3,1}^{(\boldsymbol{x})}$ & $\{0,9\}$ & 1,193 & 0,120 & 1,078 & 0,081 \\
\hline$\lambda_{4,1}^{(\boldsymbol{x})}$ & $\{1,2\}$ & 1,383 & 0,139 & 1,267 & 0,096 \\
\hline$\lambda_{5,2}^{(\boldsymbol{x})}$ & $\{-0,7\}$ & $-0,003$ & 0,841 & 0,110 & 0,339 \\
\hline$\lambda_{6,2}^{(\boldsymbol{x})}$ & $\{1,8\}$ & 0,503 & 0,667 & 1,038 & 0,454 \\
\hline$\lambda_{7,2}^{(\boldsymbol{x})}$ & $\{0,5\}$ & 0,418 & 0,467 & 0,968 & 0,354 \\
\hline$\lambda_{8,2}^{(x)}$ & $\{-0,2\}$ & 0,173 & 0,580 & 0,355 & 0,270 \\
\hline$\phi_{1,2}$ & $\{-0,2\}$ & 0,163 & 0,534 & 0,378 & 0,268 \\
\hline$\theta_{1,1}^{(\boldsymbol{\delta})}$ & $\{1,0\}$ & 0,873 & 0,138 & 0,877 & 0,105 \\
\hline$\theta_{2,2}^{(\tilde{\boldsymbol{d}})}$ & $\{1,0\}$ & 0,803 & 0,141 & 0,810 & 0,123 \\
\hline$\theta_{3,3}^{(\boldsymbol{\delta})}$ & $\{1,0\}$ & 0,743 & 0,191 & 0,782 & 0,132 \\
\hline$\theta_{4,4}^{(\boldsymbol{\delta})}$ & $\{1,0\}$ & 0,863 & 0,247 & 0,980 & 0,163 \\
\hline$\theta_{5,5}^{(\boldsymbol{\delta})}$ & $\{1,0\}$ & 1,010 & 0,549 & 1,467 & 0,280 \\
\hline$\theta_{6,6}^{(\boldsymbol{\delta})}$ & $\{1,0\}$ & 3,492 & 0,690 & 3,400 & 0,602 \\
\hline$\theta_{7,7}^{(\boldsymbol{\delta})}$ & $\{1,0\}$ & 0,896 & 0,473 & 0,424 & 0,428 \\
\hline \multirow[t]{2}{*}{$\begin{array}{r}\theta_{8,8}^{(\boldsymbol{\delta})} \\
\end{array}$} & $\{1,0\}$ & 0,889 & 0,252 & 1,032 & 0,201 \\
\hline & & \multicolumn{2}{|c|}{$n=300$} & \multicolumn{2}{|c|}{$n=500$} \\
\hline \multirow{3}{*}{$\begin{array}{c}\text { Parâmetro } \\
\lambda_{1,1}^{(\boldsymbol{x})} \\
\lambda_{(\boldsymbol{x})}\end{array}$} & \multirow{2}{*}{$\begin{array}{c}\text { Referência } \\
\quad\{0,3\}\end{array}$} & $\hat{\mu}_{\hat{\theta}}$ & $\hat{\sigma}_{\hat{\theta}}$ & $\hat{\mu}_{\hat{\theta}}$ & $\hat{\sigma}_{\hat{\theta}}$ \\
\hline & & 0,647 & 0,064 & 0,556 & 0,042 \\
\hline & $\{0,6\}$ & 0,880 & 0,059 & 0,834 & 0,046 \\
\hline$\lambda_{3,1}^{(x)}$ & $\{0,9\}$ & 1,106 & 0,070 & 1,102 & 0,052 \\
\hline$\lambda_{4,1}^{(\boldsymbol{x})}$ & $\{1,2\}$ & 1,258 & 0,079 & 1,283 & 0,060 \\
\hline$\lambda_{5,2}^{(x)}$ & $\{-0,7\}$ & $-0,493$ & 0,681 & $-0,297$ & 0,638 \\
\hline$\lambda_{6,2}^{(x)}$ & $\{1,8\}$ & 0,793 & 0,449 & 0,824 & 0,454 \\
\hline$\lambda_{7,2}^{(x)}$ & $\{0,5\}$ & 0,447 & 0,524 & 0,628 & 0,624 \\
\hline$\lambda_{8,2}^{(\boldsymbol{x})}$ & $\{-0,2\}$ & $-0,121$ & 0,450 & $-0,008$ & 0,428 \\
\hline$\phi_{1,2}$ & $\{-0,2\}$ & $-0,094$ & 0,436 & 0,024 & 0,428 \\
\hline$\theta_{1,1}^{(\boldsymbol{\delta})}$ & $\{1,0\}$ & 1,192 & 0,087 & 0,805 & 0,061 \\
\hline$\theta_{2,2}^{(\boldsymbol{\delta})}$ & $\{1,0\}$ & 0,757 & 0,090 & 0,748 & 0,066 \\
\hline$\theta_{3,3}^{(\boldsymbol{\delta})}$ & $\{1,0\}$ & 0,765 & 0,109 & 0,724 & 0,082 \\
\hline$\theta_{4,4}^{(\boldsymbol{\delta})}$ & $\{1,0\}$ & 0,965 & 0,128 & 0,926 & 0,099 \\
\hline$\theta_{5,5}^{(\boldsymbol{\delta})}$ & $\{1,0\}$ & 0,915 & 0,608 & 1,115 & 0,581 \\
\hline$\theta_{6,6}^{(\widetilde{\delta})}$ & $\{1,0\}$ & 3,528 & 0,546 & 3,542 & 0,516 \\
\hline$\theta_{7.7}^{(\boldsymbol{\delta})}$ & $\{1,0\}$ & 0,778 & 0,520 & 0,656 & 0,622 \\
\hline$\theta_{8.8}^{(\boldsymbol{\delta})}$ & $\{1,0\}$ & 0,950 & 0,163 & 0,930 & 0,153 \\
\hline
\end{tabular}


Tabela 5.11: Estatisticas dos mil valores obtidos via simulação para os indicadores clássicos e robustos do MEE descrito pela Figura 5.1, por tamanho de amostra e com fração de $25 \%$ de contaminação proveniente da lei $t$-Student com cinco graus de liberdade.

\begin{tabular}{|c|c|c|c|c|c|c|c|c|c|}
\hline \multirow{3}{*}{$\begin{array}{l}\text { Îndice } \\
\text { SRMR }\end{array}$} & \multirow{3}{*}{$\begin{array}{c}\text { Referência } \\
{[0 ; 0,08[}\end{array}$} & \multicolumn{4}{|c|}{$n=100$} & \multicolumn{4}{|c|}{$n=200$} \\
\hline & & $\hat{\mu}$ & $\hat{\sigma}$ & $\hat{\pi}$ & $\hat{\sigma}_{\hat{\pi}}$ & $\hat{\mu}$ & $\hat{\sigma}$ & $\hat{\pi}$ & $\hat{\sigma}_{\hat{\pi}}$ \\
\hline & & 0,123 & 0,023 & 0,992 & 0,089 & 0,145 & 0,022 & 1,000 & 0,000 \\
\hline RMSEA & {$[0 ; 0,08[$} & 0,153 & 0,023 & 0,997 & 0,055 & 0,179 & 0,016 & 1,000 & 0,000 \\
\hline NFI & ] $0,90 ; 1]$ & 0,739 & 0,048 & 1,000 & 0,000 & 0,723 & 0,038 & 1,000 & 0,000 \\
\hline IFI & ] $0,90 ; 1]$ & 0,802 & 0,052 & 0,999 & 0,032 & 0,751 & 0,040 & 1,000 & 0,000 \\
\hline NNFI & ] $0,90 ; 1]$ & 0,696 & 0,080 & 0,999 & 0,032 & 0,626 & 0,059 & 1,000 & 0,000 \\
\hline CFI & ] $0,90 ; 1]$ & 0,793 & 0,054 & 0,999 & 0,032 & 0,746 & 0,040 & 1,000 & 0,000 \\
\hline RNI & ]0,90;1] & 0,793 & 0,054 & 0,999 & 0,032 & 0,746 & 0,040 & 1,000 & 0,000 \\
\hline $\mathrm{GFI}_{\mathrm{MV}}$ & ] $0,90 ; 1]$ & 0,866 & 0,024 & 1,000 & 0,000 & 0,851 & 0,019 & 1,000 & 0,000 \\
\hline $\mathrm{GFI}_{\mathrm{MV}}^{\mathrm{GKO}}$ & ] $0,90 ; 1]$ & 0,785 & 0,096 & 0,999 & 0,032 & 0,796 & 0,062 & 1,000 & 0,000 \\
\hline $\mathrm{GFI}_{\mathrm{MV}}^{\mathrm{EVM}}$ & ] $0,90 ; 1]$ & 0,868 & 0,046 & 1,000 & 0,000 & 0,877 & 0,042 & 0,995 & 0,071 \\
\hline $\mathrm{GFI}_{\mathrm{MV}}^{\mathrm{CDM}}$ & ] $0,90 ; 1]$ & 0,839 & 0,039 & 1,000 & 0,000 & 0,867 & 0,029 & 1,000 & 0,000 \\
\hline $\mathrm{GFI}_{\mathrm{MV}}^{\mathrm{S}}$ & ] $0,90 ; 1]$ & 0,893 & 0,033 & 0,989 & 0,104 & 0,892 & 0,025 & 0,998 & 0,045 \\
\hline $\mathrm{GFI}_{\mathrm{MV}}^{\mathrm{MM}}$ & ] $0,90 ; 1]$ & 0,890 & 0,033 & 0,986 & 0,118 & 0,893 & 0,024 & 0,998 & 0,045 \\
\hline $\mathrm{AGFI}_{\mathrm{MV}}$ & ] $0,90 ; 1]$ & 0,747 & 0,046 & 1,000 & 0,000 & 0,718 & 0,036 & 1,000 & 0,000 \\
\hline $\mathrm{AGFI}_{\mathrm{MV}}^{\mathrm{GKO}}$ & ] $0,90 ; 1]$ & 0,592 & 0,183 & 1,000 & 0,000 & 0,614 & 0,118 & 1,000 & 0,000 \\
\hline $\mathrm{AGFI}_{\mathrm{MV}}^{\mathrm{EVM}}$ & ] $0,90 ; 1]$ & 0,750 & 0,086 & 1,000 & 0,000 & 0,767 & 0,080 & 1,000 & 0,000 \\
\hline $\mathrm{AGFI}_{\mathrm{MV}}^{\mathrm{CDM}}$ & ] $0,90 ; 1]$ & 0,694 & 0,074 & 1,000 & 0,000 & 0,749 & 0,054 & 1,000 & 0,000 \\
\hline $\mathrm{AGFI}_{\mathrm{MV}}^{\mathrm{S}}$ & ] $0,90 ; 1]$ & 0,798 & 0,063 & 0,999 & 0,032 & 0,796 & 0,048 & 1,000 & 0,000 \\
\hline \multirow[t]{2}{*}{$\mathrm{AGFI}_{\mathrm{MV}}^{\mathrm{MM}}$} & ] $0,90 ; 1]$ & 0,792 & 0,063 & 0,999 & 0,032 & 0,797 & 0,046 & 1,000 & 0,000 \\
\hline & & \multicolumn{4}{|c|}{$n=300$} & \multicolumn{4}{|c|}{$n=500$} \\
\hline Índice & Referência & $\hat{\mu}$ & $\hat{\sigma}$ & $\hat{\pi}$ & $\hat{\sigma}_{\hat{\pi}}$ & $\hat{\mu}$ & $\hat{\sigma}$ & $\hat{\pi}$ & $\hat{\sigma}_{\hat{\pi}}$ \\
\hline SRMR & {$[0 ; 0,08[$} & 0,132 & 0,018 & 1,000 & 0,000 & 0,130 & 0,014 & 1,000 & 0,000 \\
\hline RMSEA & {$[0 ; 0,08[$} & 0,164 & 0,011 & 1,000 & 0,000 & 0,163 & 0,009 & 1,000 & 0,000 \\
\hline NFI & ]0,90;1] & 0,743 & 0,029 & 1,000 & 0,000 & 0,758 & 0,022 & 1,000 & 0,000 \\
\hline IFI & ] $0,90 ; 1]$ & 0,765 & 0,029 & 1,000 & 0,000 & 0,771 & 0,022 & 1,000 & 0,000 \\
\hline NNFI & ] $0,90 ; 1]$ & 0,649 & 0,044 & 1,000 & 0,000 & 0,659 & 0,033 & 1,000 & 0,000 \\
\hline CFI & ] $0,90 ; 1]$ & 0,762 & 0,030 & 1,000 & 0,000 & 0,769 & 0,022 & 1,000 & 0,000 \\
\hline RNI & ] $0,90 ; 1]$ & 0,762 & 0,030 & 1,000 & 0,000 & 0,769 & 0,022 & 1,000 & 0,000 \\
\hline $\mathrm{GFI}_{\mathrm{MV}}$ & ]0,90;1] & 0,873 & 0,013 & 1,000 & 0,000 & 0,881 & 0,011 & 1,000 & 0,000 \\
\hline $\mathrm{GFI}_{\mathrm{MV}}^{\mathrm{GKO}}$ & ] $0,90 ; 1]$ & 0,847 & 0,037 & 1,000 & 0,000 & 0,863 & 0,026 & 1,000 & 0,000 \\
\hline $\mathrm{GFI}_{\mathrm{MV}}^{\mathrm{EVM}}$ & ] $0,90 ; 1]$ & 0,905 & 0,023 & 0,996 & 0,063 & 0,910 & 0,018 & 0,997 & 0,055 \\
\hline $\mathrm{GFI}_{\mathrm{MV}}^{\mathrm{CDM}}$ & ] $0,90 ; 1]$ & 0,859 & 0,022 & 1,000 & 0,000 & 0,856 & 0,020 & 1,000 & 0,000 \\
\hline $\mathrm{GFI}_{\mathrm{MV}}^{\mathrm{S}}$ & ] $0,90 ; 1]$ & 0,912 & 0,017 & 1,000 & 0,000 & 0,915 & 0,012 & 1,000 & 0,000 \\
\hline $\mathrm{GFI}_{\mathrm{MV}}^{\mathrm{MM}}$ & ] $0,90 ; 1]$ & 0,913 & 0,017 & 0,999 & 0,032 & 0,917 & 0,012 & 1,000 & 0,000 \\
\hline $\mathrm{AGFI}_{\mathrm{MV}}$ & ] $0,90 ; 1]$ & 0,760 & 0,025 & 1,000 & 0,000 & 0,775 & 0,020 & 1,000 & 0,000 \\
\hline $\mathrm{AGFI}_{\mathrm{MV}}^{\mathrm{GKO}}$ & ] $0,90 ; 1]$ & 0,710 & 0,069 & 1,000 & 0,000 & 0,740 & 0,049 & 1,000 & 0,000 \\
\hline $\mathrm{AGFI}_{\mathrm{MV}}^{\mathrm{EVM}}$ & ] $0,90 ; 1]$ & 0,820 & 0,043 & 1,000 & 0,000 & 0,830 & 0,034 & 1,000 & 0,000 \\
\hline $\mathrm{AGFI}_{\mathrm{MV}}^{\mathrm{CDM}}$ & ] $0,90 ; 1]$ & 0,732 & 0,042 & 1,000 & 0,000 & 0,727 & 0,038 & 1,000 & 0,000 \\
\hline $\mathrm{AGFI}_{\mathrm{MV}}^{\mathrm{S}}$ & ] $0,90 ; 1]$ & 0,833 & 0,033 & 1,000 & 0,000 & 0,840 & 0,023 & 1,000 & 0,000 \\
\hline $\mathrm{AGFI}_{\mathrm{MV}}^{\mathrm{MM}}$ & ] $0,90 ; 1]$ & 0,834 & 0,033 & 1,000 & 0,000 & 0,843 & 0,022 & 1,000 & 0,000 \\
\hline
\end{tabular}




\subsubsection{Cenário 2: Análise Fatorial Confirmatória - poucos parâmetros a estimar}

Para esta subseção, foi tomado um modelo de Análise Fatorial Confirmatória que possui uma variável latente exógena operacionalizada por quatro variáveis observadas exógenas - todas gaussianas -, conforme ilustrado na Figura 5.2. Diferente do que foi feito no Cenário 1, optou-se por admitir para o Cenário 2 que $\lambda_{1,1}^{(\boldsymbol{x})}=1,0$ (i.e., esse coeficiente não foi estimado), ao invés de identificar um valor para a variância de $\xi_{1}$ (ou seja, $\phi_{1,1}$ foi estimado).

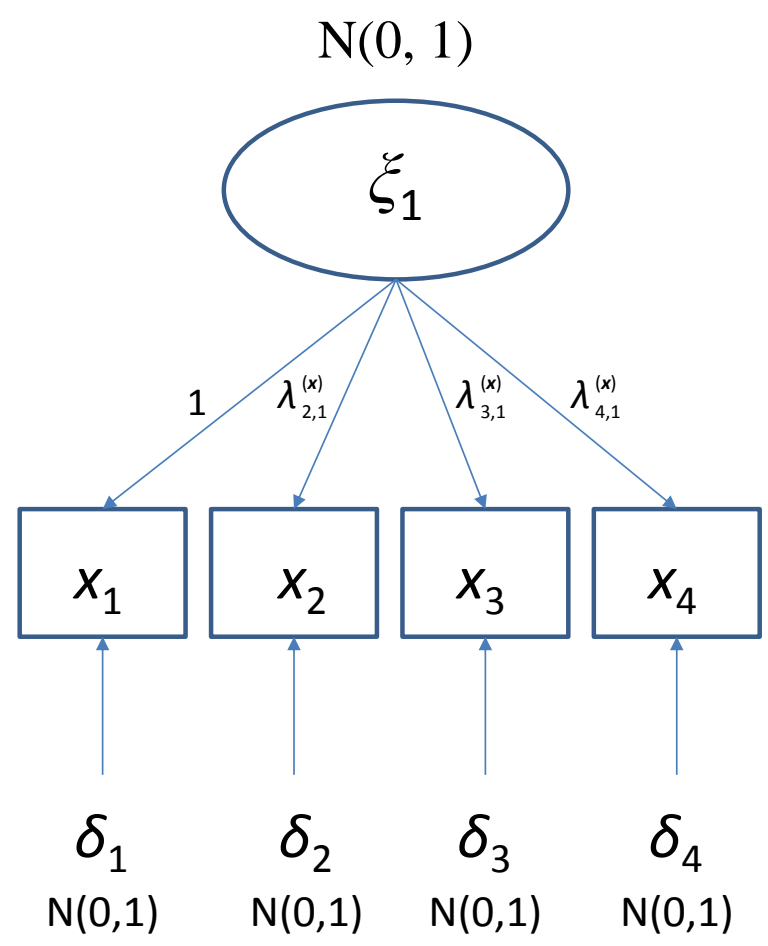

Figura 5.2: Modelo de Análise Fatorial Confirmatória para simulação do Cenário 2.

Esse modelo possui as seguintes equações para o submodelo de mensuração em $\boldsymbol{x}$ :

$$
\begin{array}{lcc}
x_{1}= & \xi_{1} & +\delta_{1} \\
x_{2}= & \lambda_{2,1}^{(\boldsymbol{x})} \xi_{1} & +\delta_{2} \\
x_{3} & =\lambda_{3,1}^{(\boldsymbol{x})} \xi_{1} & +\delta_{3} \\
x_{4} & =\lambda_{4,1}^{(\boldsymbol{x})} \xi_{1} & +\delta_{4}
\end{array}
$$

ou, usando matrizes,

$$
\left[\begin{array}{l}
x_{1} \\
x_{2} \\
x_{3} \\
x_{4}
\end{array}\right]=\left[\begin{array}{c}
1 \\
\lambda_{2,1}^{(\boldsymbol{x})} \\
\lambda_{3,1}^{(\boldsymbol{x})} \\
\lambda_{4,1}^{(\boldsymbol{x})}
\end{array}\right]\left[\xi_{1}\right]+\left[\begin{array}{c}
\delta_{1} \\
\delta_{2} \\
\delta_{3} \\
\delta_{4}
\end{array}\right]
$$


com

$$
\boldsymbol{\Theta}_{\boldsymbol{\delta}}=\left[\begin{array}{cccc}
\theta_{1,1}^{(\boldsymbol{\delta})} & 0 & 0 & 0 \\
0 & \theta_{2,2}^{(\boldsymbol{\delta})} & 0 & 0 \\
0 & 0 & \theta_{3,3}^{(\boldsymbol{\delta})} & 0 \\
0 & 0 & 0 & \theta_{4,4}^{(\boldsymbol{\delta})}
\end{array}\right]
$$

$$
\mathbf{\Phi}=\left[\phi_{1,1}\right]
$$

Para efeitos de comparação, foram arbitrados os seguintes valores: $\lambda_{2,1}^{(\boldsymbol{x})}=4,0, \lambda_{3,1}^{(\boldsymbol{x})}=2,0$, $\lambda_{4,1}^{(\boldsymbol{x})}=5,0$ e $\phi_{1,1}=\theta_{1,1}^{(\boldsymbol{\delta})}=\theta_{2,2}^{(\boldsymbol{\delta})}=\theta_{3,3}^{(\boldsymbol{\delta})}=\theta_{4,4}^{(\boldsymbol{\delta})}=1$.

Para contaminar as variáveis observadas exógenas $x_{1}, x_{2}, x_{3}$ e $x_{4}, \alpha \cdot n$ valores de $\delta_{1}, \delta_{2}, \delta_{3}$ e $\delta_{4}$ foram substituídos por outliers, respectivamente. No que é tocante aos desvios de simetria, $\alpha \cdot n$ pontos de cada uma das quatro variáveis de erro foram gerados a partir da lei Log-Normal Padrão, pela função $\operatorname{rlnorm}(\alpha \cdot n, 0,1)$ do $\mathrm{R}$; quanto aos desvios de curtose, as gerações foram analogamente realizadas a partir da lei Uniforme Contínua com suporte no intervalo ]-5; 5[, usando a função runif $(\alpha \cdot n,-5,5)$ do R. Este esquema está resumido na Tabela 5.12.

Tabela 5.12: Esquema para a geração de outliers no Cenário 2.

\begin{tabular}{c|c|c|c}
\hline$n$ & $\alpha$ & $\alpha \cdot n$ & Variáveis perturbadas \\
\hline \hline 100 & 0,05 & 5 & \\
\hline 100 & 0,25 & 25 & \\
\hline 200 & 0,05 & 10 & $\delta_{1}$ \\
\hline 200 & 0,25 & 50 & $\delta_{2}$ \\
\hline 300 & 0,05 & 15 & $\delta_{3}$ \\
\hline 300 & 0,25 & 75 & $\delta_{4}$ \\
\hline 500 & 0,05 & 25 & \\
\hline 500 & 0,25 & 125 & \\
\hline
\end{tabular}

O programa do Cenário 2 contaminado por $\alpha \cdot n=5$ outliers provenientes da lei Log-Normal Padrão está disponível na Seção C.2 do Apêndice C.

\section{Resultados e discussão}

A escolha do MEE da Figura 5.2 visou controlar vícios de interpretação relacionados à quantidade de parâmetros, para que a comparação com o primeiro estudo de simulação aqui apresentado permita aferir se a capacidade de detecção dos indicadores em captar problemas nas estimativas relacionados a outliers muda quando se tem mais ou menos parâmetros a serem estimados.

As estimativas dos parâmetros e indicadores do modelo sob circunstâncias normais (Seção 2.6) estão apresentadas na Tabela 5.13 e na Tabela 5.14, respectivamente.

É interessante notar que as estimativas estão, em média, muito próximas de seus valores fixados já mesmo para $n=100$, expressando que esse tamanho de amostra é adequado para conduzir o ajuste desses oito parâmetros. A variabilidade reduz-se com o crescimento do número de observações, pelo que se repara nos desvios-padrão.

Uma vez que as condições de regularidade estão atendidas e as médias das estimativas dos parâmetros se aproximam de seus verdadeiros valores, verificou-se algo que era esperado: a maioria dos valores médios dos indicadores recomenda o ajuste. No entanto, as medidas $\mathrm{AGFI}_{\mathrm{MV}}^{\mathrm{GKO}}, \mathrm{AGFI}_{\mathrm{MV}}^{\mathrm{EVM}}$ 
e $\mathrm{AGFI}_{\mathrm{MV}}^{\mathrm{CDM}}$ erroneamente conduzem ao diagnóstico de que a modelagem realizada não foi satisfatória, portanto não serão mais mencionadas nas análises deste modelo. Isso sinaliza que os índices $\mathrm{GFI}_{\mathrm{MV}}^{\mathrm{GKO}}, \mathrm{GFI}_{\mathrm{MV}}^{\mathrm{EVM}}$ e $\mathrm{GFI}_{\mathrm{MV}}^{\mathrm{CDM}}$ são preferíveis para lidar com uma quantidade de parâmetros menor.

Os resultados médios sobre o ajuste encontrados para o indicador $\mathrm{GFI}_{\mathrm{MV}}^{\mathrm{GKO}}$ são interpretados com base na Subseção 4.1.8 como segue: bons, para $n<300$; e muito bons, para $n \geq 300$. A impressão é a de que esse índice é bastante sensível a suaves variações na amostra providas pelo acaso (no caso, a ligeiros desvios naturais do processo computacional da geração pseudoaleatória dos dados), algo que pode ser percebido ao comparar seu desvio-padrão com o das outras medidas.

A Tabela 5.15 mostra que a fração de $5 \%$ de contaminação proveniente da lei Log-Normal Padrão provoca afastamentos nas médias das estimativas dos parâmetros em relação aos valores fixados, nos quatro tamanhos de amostra aqui considerados.

Mesmo a menores quantidades de observações $(n \leq 100)$, esses desvios nas médias das estimativas dos parâmetros não são facilmente identificados pelos indicadores (Tabela 5.16). Felizmente, um exame cuidadoso das diferenças entre os números exibidos na Tabela 5.14 e na Tabela 5.16 pode evidenciar suspeitas de mau ajustamento ao pesquisador.

Para $n>300$, o indicador $\mathrm{GFI}_{\mathrm{MV}}^{\mathrm{GKO}}$ aponta que o ajuste está muito bom quando $\alpha=0$ e, sob a fração $\alpha=0,05$ de contaminação pela mencionada distribuição assimétrica positiva, sugere que o ajuste está somente bom. Ainda que o índice $\mathrm{GFI}_{\mathrm{MV}}^{\mathrm{GKO}}$ conduza sempre à conclusão de que o modelo está bom com ou sem contaminação para $n \leq 300$, a diferença absoluta das médias de seus valores nesses dois casos está em torno de 0,01. As demais medidas erroneamente sugerem que o ajuste está praticamente perfeito, com suas médias diferindo em módulo por volta de 0,001 nas duas situações.

Embora o desempenho dos índices $\mathrm{GFI}_{\mathrm{MV}}^{\mathrm{EVM}}$ e $\mathrm{GFI}_{\mathrm{MV}}^{\mathrm{CDM}}$ tenha sido superior ou, ao menos, equivalente a $\mathrm{GFI}_{\mathrm{MV}}$ como previsto (Cirillo e Barroso, 2012a), o uso de qualquer um desses três indicadores não resolveria o problema exposto. Além disso, verificou-se mais uma vez que as adaptações $\mathrm{GFI}_{\mathrm{MV}}^{\mathrm{S}}$ e $\mathrm{GFI}_{\mathrm{MV}}^{\mathrm{MM}}$ são piores em relação ao usual $\mathrm{GFI}_{\mathrm{MV}}$, apesar de os métodos robustos $\mathrm{S}$ e MM possuírem elevados valores para seus pontos de ruptura e suas eficiências assintóticas sob normalidade.

Subindo a contaminação pela lei Log-Normal Padrão para a fração de 25\%, as médias das estimativas dos parâmetros passam a ser severamente deslocadas de seus verdadeiros valores (Tabela 5.17). Ainda assim, nem todos os indicadores apresentaram resultados fidedignos quanto à detecção desta avaria, especialmente para elevadas quantidades de observações (Tabela 5.18). A saber, apenas os índices RMSEA, $\mathrm{GFI}_{\mathrm{MV}}^{\mathrm{GKO}}$ e $\mathrm{AGFI}_{\mathrm{MV}}$ foram precisos ao diagnosticar mau ajuste em todos os quatro tamanhos de amostra considerados.

Do mesmo modo que o percentual de $5 \%$ de contaminação da lei Log-Normal Padrão afetou os resultados, a Tabela 5.19 mostra que as médias das estimativas de parâmetros sofrem translações em relação a seus valores populacionais quando se considera a proporção de $5 \%$ de contaminação pela lei Uniforme Contínua em $]-5 ; 5[$.

Independentemente da mistura distribucional focar em desvio de simetria ou excesso de curtose, praticamente todos os indicadores não conseguiram captar os impactos causados pela fração de contaminação de 5\%. Por sorte, a interpretação aqui aplicada aos valores da medida $\mathrm{GFI}_{\mathrm{MV}}^{\mathrm{GKO}}$ contidos na Tabela 5.16 também é cabível à Tabela 5.20.

A Tabela 5.21 mostra que as médias das estimativas dos parâmetros estão altamente desviadas de seus verdadeiros valores quando se toma a fração de $25 \%$ de contaminação pela lei Uniforme Contínua em ] $-5 ; 5[$, o que era de se imaginar, visto que já foram observadas distorções quando a 
proporção de valores obtidos por aquela lei de probabilidade era de apenas 5\%. Pela Tabela 5.22, checa-se que o indicador $\mathrm{GFI}_{\mathrm{MV}}^{\mathrm{GKO}}$ pode insinuar mau ajuste analisando seus valores médios por faixas, tal como foi feito ao comparar a Tabela $5.16 \mathrm{com}$ a Tabela 5.14. No entanto, os índices RMSEA e $\mathrm{AGFI}_{\mathrm{MV}}$ proporcionaram diagnósticos bem precisos e claros neste caso.

De modo geral, a comparação entre os dois cenários transmite a impressão de que a quantidade de variáveis observadas contaminadas influencia bastante o desempenho dos indicadores. Mesmo com frações de contaminação elevadas, a resposta dada pelos índices no Cenário 2 foi bem menos expressiva que no Cenário 1. Essa também parece ser uma explicação plausível para o fato de a medida NFI não ter tido bom desempenho no Cenário 2, quando se esperava o contrário por conta de menos parâmetros terem sido estimados nesse segundo estudo de simulação, uma vez que sua sensibilidade à adição de parâmetros ao MEE foi levantada na Subseção 4.1.3.

Diferente do que ocorreu com a medida NFI, o indicador RMSEA desempenhou melhor no Cenário 2. Isso sinaliza que esse índice seja mais sensível para captar perturbações ocorridas em uma quantidade de variáveis observadas menor, desde que o número de parâmetros a estimar não seja elevado. A saber, ele vem sendo considerado como uma das medidas mais informativas sobre a qualidade do ajuste (Diamantopoulos e Siguaw, 2000).

Avaliando os resultados por tipo de mistura de distribuição (desvio de simetria e excesso de curtose), fração de contaminação $(\alpha \in\{0,05,0,25\})$, tamanho de amostra $(n \in\{100,200,300,500\})$ e quantidade de variáveis observadas perturbadas ( 8 no Cenário 1 e 4 no Cenário 2) simultaneamente, as medidas $\mathrm{GFI}_{\mathrm{MV}}^{\mathrm{GKO}}$ e $\mathrm{AFI}_{\mathrm{MV}}^{\mathrm{GKO}}$ foram as que únicas que conseguiram ser informativas em todas essas situações, devendo-se escolher a primeira ou a segunda quando a quantidade de parâmetros a serem estimados é baixa ou elevada, respectivamente.

Entretanto, o uso dos índices $\mathrm{GFI}_{\mathrm{MV}}^{\mathrm{GKO}}$ e $\mathrm{AFI}_{\mathrm{MV}}^{\mathrm{GKO}}$ não deve ser feito de maneira isolada. Como a leitura de seus desvios-padrão sugeriu heterogeneidade e, para $n \leq 300$, valores dentro do intervalo aberto ]0,90;0,93[ podem ser difíceis de interpretar (inconclusivos à primeira vista), recomendase fortemente que o pesquisador recorra a outras técnicas de análise estatística para dar mais fundamento aos diagnósticos que esses dois indicadores venham a fornecer. 
Tabela 5.13: Estatísticas dos mil valores obtidos via simulação para as estimativas dos parâmetros do MEE descrito pela Figura 5.2, por tamanho de amostra e com fração de contaminação zero.

\begin{tabular}{|c|c|c|c|c|c|}
\hline \multirow{10}{*}{$\begin{array}{c}\text { Parâmetro } \\
\qquad \lambda_{2,1}^{(\boldsymbol{x})} \\
\lambda_{3,1}^{(\boldsymbol{x})} \\
\lambda_{4,1}^{(\boldsymbol{x})} \\
\phi_{1,1} \\
\theta_{1,1}^{(\boldsymbol{\delta})} \\
\theta_{2,2}^{(\boldsymbol{\delta})} \\
\theta_{3,3}^{(\boldsymbol{\delta})} \\
\theta_{4,4}^{(\boldsymbol{\delta})} \\
\end{array}$} & \multirow{3}{*}{$\begin{array}{c}\text { Referência } \\
\qquad\{4,0\}\end{array}$} & \multicolumn{2}{|c|}{$n=100$} & \multicolumn{2}{|c|}{$n=200$} \\
\hline & & $\hat{\mu}_{\hat{\theta}}$ & $\hat{\sigma}_{\hat{\theta}}$ & $\hat{\mu}_{\hat{\theta}}$ & $\hat{\sigma}_{\hat{\theta}}$ \\
\hline & & 4,049 & 0,446 & 4,031 & 0,309 \\
\hline & $\{2,0\}$ & 2,024 & 0,246 & 2,010 & 0,162 \\
\hline & $\{5,0\}$ & 5,055 & 0,564 & 5,033 & 0,383 \\
\hline & $\{1,0\}$ & 1,006 & 0,248 & 1,002 & 0,172 \\
\hline & $\{1,0\}$ & 0,993 & 0,139 & 0,993 & 0,104 \\
\hline & $\{1,0\}$ & 0,991 & 0,285 & 0,987 & 0,200 \\
\hline & $\{1,0\}$ & 0,990 & 0,153 & 0,994 & 0,114 \\
\hline & $\{1,0\}$ & 1,013 & 0,406 & 0,992 & 0,285 \\
\hline \multirow{5}{*}{$\begin{array}{c}\text { Parâmetro } \\
\qquad \begin{array}{c}\lambda_{2,1}^{(\boldsymbol{x})} \\
\lambda_{3,1}^{(\boldsymbol{x})} \\
\lambda_{4,1}^{(\boldsymbol{x})}\end{array}\end{array}$} & \multirow{3}{*}{$\begin{array}{c}\text { Referência } \\
\quad\{4,0\}\end{array}$} & \multicolumn{2}{|c|}{$n=300$} & \multicolumn{2}{|c|}{$n=500$} \\
\hline & & $\hat{\mu}_{\hat{\theta}}$ & $\hat{\sigma}_{\hat{\theta}}$ & $\hat{\mu}_{\hat{\theta}}$ & $\hat{\sigma}_{\hat{\theta}}$ \\
\hline & & 4,004 & 0,247 & 4,011 & 0,188 \\
\hline & $\{2,0\}$ & 2,002 & 0,131 & 2,004 & 0,101 \\
\hline & $\{5,0\}$ & 5,010 & 0,302 & 5,015 & 0,231 \\
\hline$\phi_{1,1}$ & $\{1,0\}$ & 1,009 & 0,142 & 1,003 & 0,110 \\
\hline$\theta_{1,1}^{(\boldsymbol{\delta})}$ & $\{1,0\}$ & 0,994 & 0,086 & 1,000 & 0,065 \\
\hline$\theta_{2,2}^{(\boldsymbol{\delta})}$ & $\{1,0\}$ & 0,999 & 0,159 & 0,997 & 0,125 \\
\hline$\theta_{3,3}^{(\boldsymbol{\delta})}$ & $\{1,0\}$ & 0,997 & 0,088 & 1,003 & 0,072 \\
\hline$\theta_{4,4}^{(\boldsymbol{\delta})}$ & $\{1,0\}$ & 0,991 & 0,229 & 0,992 & 0,179 \\
\hline
\end{tabular}


Tabela 5.14: Estatísticas dos mil valores obtidos via simulação para os indicadores clássicos e robustos do MEE descrito pela Figura 5.2, por tamanho de amostra e com fração de contaminação zero.

\begin{tabular}{|c|c|c|c|c|c|c|c|c|c|}
\hline \multirow[b]{2}{*}{ Índice } & \multirow[b]{2}{*}{ Referência } & \multicolumn{4}{|c|}{$n=100$} & \multicolumn{4}{|c|}{$n=200$} \\
\hline & & $\hat{\mu}$ & $\hat{\sigma}$ & $\hat{\pi}$ & $\hat{\sigma}_{\hat{\pi}}$ & $\hat{\mu}$ & $\hat{\sigma}$ & $\hat{\pi}$ & $\hat{\sigma}_{\hat{\pi}}$ \\
\hline SRMR & {$[0 ; 0,08[$} & 0,009 & 0,006 & 0,000 & 0,000 & 0,006 & 0,004 & 0,000 & 0,000 \\
\hline RMSEA & {$[0 ; 0,08[$} & 0,034 & 0,051 & 0,213 & 0,410 & 0,024 & 0,036 & 0,101 & 0,301 \\
\hline NFI & ] $0,90 ; 1]$ & 0,995 & 0,004 & 0,000 & 0,000 & 0,998 & 0,002 & 0,000 & 0,000 \\
\hline IFI & ] $0,90 ; 1]$ & 1,000 & 0,004 & 0,000 & 0,000 & 1,000 & 0,002 & 0,000 & 0,000 \\
\hline NNFI & ] $0,90 ; 1]$ & 1,000 & 0,013 & 0,010 & 0,100 & 1,000 & 0,006 & 0,000 & 0,000 \\
\hline CFI & ] $0,90 ; 1]$ & 0,998 & 0,003 & 0,000 & 0,000 & 0,999 & 0,002 & 0,000 & 0,000 \\
\hline RNI & ] $0,90 ; 1]$ & 1,000 & 0,004 & 0,000 & 0,000 & 1,000 & 0,002 & 0,000 & 0,000 \\
\hline $\mathrm{GFI}_{\mathrm{MV}}$ & ] $0,90 ; 1]$ & 0,990 & 0,009 & 0,005 & 0,071 & 0,995 & 0,005 & 0,000 & 0,000 \\
\hline $\mathrm{GFI}_{\mathrm{MV}}^{\mathrm{GKO}}$ & ] $0,90 ; 1]$ & 0,916 & 0,108 & 0,491 & 0,500 & 0,937 & 0,060 & 0,472 & 0,499 \\
\hline $\mathrm{GFI}_{\mathrm{MV}}^{\mathrm{EVM}}$ & $0,90 ; 1]$ & 0,974 & 0,047 & 0,137 & 0,344 & 0,990 & 0,018 & 0,030 & 0,171 \\
\hline $\mathrm{GFI}_{\mathrm{MV}}^{\mathrm{CDM}}$ & ] $0,90 ; 1]$ & 0,977 & 0,037 & 0,114 & 0,318 & 0,992 & 0,015 & 0,013 & 0,113 \\
\hline $\mathrm{GFI}_{\mathrm{MV}}^{\mathrm{S}}$ & ] $0,90 ; 1]$ & 0,990 & 0,017 & 0,031 & 0,173 & 0,995 & 0,008 & 0,004 & 0,063 \\
\hline $\mathrm{GFI}_{\mathrm{MV}}^{\mathrm{MM}}$ & ] $0,90 ; 1]$ & 0,998 & 0,003 & 0,000 & 0,000 & 0,999 & 0,002 & 0,000 & 0,000 \\
\hline $\mathrm{AGFI}_{\mathrm{MV}}$ & ] $0,90 ; 1]$ & 0,950 & 0,046 & 0,392 & 0,488 & 0,975 & 0,023 & 0,135 & 0,342 \\
\hline $\mathrm{AGFI}_{\mathrm{MV}}^{\mathrm{GKO}}$ & ] $0,90 ; 1]$ & 0,579 & 0,541 & 0,839 & 0,368 & 0,685 & 0,299 & 0,910 & 0,286 \\
\hline $\mathrm{AGFI}_{\mathrm{MV}}^{\mathrm{EVM}}$ & ] $0,90 ; 1]$ & 0,869 & 0,237 & 0,562 & 0,496 & 0,948 & 0,088 & 0,301 & 0,459 \\
\hline $\mathrm{AGFI}_{\mathrm{MV}}^{\mathrm{CDM}}$ & ] $0,90 ; 1]$ & 0,884 & 0,187 & 0,543 & 0,498 & 0,960 & 0,073 & 0,230 & 0,421 \\
\hline $\mathrm{AGFI}_{\mathrm{MV}}^{\mathrm{S}}$ & ] $0,90 ; 1]$ & 0,949 & 0,085 & 0,285 & 0,452 & 0,977 & 0,039 & 0,116 & 0,320 \\
\hline \multirow[t]{2}{*}{$\mathrm{AGFI}_{\mathrm{MV}}^{\mathrm{MM}}$} & ] $0,90 ; 1]$ & 0,991 & 0,017 & 0,038 & 0,191 & 0,995 & 0,009 & 0,005 & 0,071 \\
\hline & & \multicolumn{4}{|c|}{$n=300$} & \multicolumn{4}{|c|}{$n=500$} \\
\hline Índice & Referência & $\hat{\mu}$ & $\hat{\sigma}$ & $\hat{\pi}$ & $\hat{\sigma}_{\hat{\pi}}$ & $\hat{\mu}$ & $\hat{\sigma}$ & $\hat{\pi}$ & $\hat{\sigma}_{\hat{\pi}}$ \\
\hline SRMR & {$[0 ; 0,08[$} & 0,005 & 0,003 & 0,000 & 0,000 & 0,004 & 0,002 & 0,000 & 0,000 \\
\hline RMSEA & {$[0 ; 0,08[$} & 0,019 & 0,030 & 0,060 & 0,238 & 0,016 & 0,023 & 0,010 & 0,100 \\
\hline NFI & ] $0,90 ; 1]$ & 0,999 & 0,002 & 0,000 & 0,000 & 0,999 & 0,001 & 0,000 & 0,000 \\
\hline IFI & ] $0,90 ; 1]$ & 1,000 & 0,002 & 0,000 & 0,000 & 1,000 & 0,001 & 0,000 & 0,000 \\
\hline NNFI & ] $0,90 ; 1]$ & 1,000 & 0,005 & 0,000 & 0,000 & 1,000 & 0,003 & 0,000 & 0,000 \\
\hline CFI & ] $0,90 ; 1]$ & 0,999 & 0,001 & 0,000 & 0,000 & 1,000 & 0,001 & 0,000 & 0,000 \\
\hline RNI & ] $0,90 ; 1]$ & 1,000 & 0,002 & 0,000 & 0,000 & 1,000 & 0,001 & 0,000 & 0,000 \\
\hline $\mathrm{GFI}_{\mathrm{MV}}$ & ] $0,90 ; 1]$ & 0,997 & 0,003 & 0,000 & 0,000 & 0,998 & 0,002 & 0,000 & 0,000 \\
\hline $\mathrm{GFI}_{\mathrm{MV}}^{\mathrm{GKO}}$ & ] $0,90 ; 1]$ & 0,946 & 0,042 & 0,426 & 0,495 & 0,950 & 0,029 & 0,426 & 0,495 \\
\hline $\mathrm{GFI}_{\mathrm{MV}}^{\mathrm{EVM}}$ & ] $0,90 ; 1]$ & 0,994 & 0,011 & 0,006 & 0,077 & 0,997 & 0,005 & 0,000 & 0,000 \\
\hline $\mathrm{GFI}_{\mathrm{MV}}^{\mathrm{CDM}}$ & ] $0,90 ; 1]$ & 0,996 & 0,008 & 0,007 & 0,083 & 0,998 & 0,003 & 0,000 & 0,000 \\
\hline $\mathrm{GFI}_{\mathrm{MV}}^{\mathrm{S}}$ & ] $0,90 ; 1]$ & 0,997 & 0,005 & 0,002 & 0,045 & 0,998 & 0,002 & 0,000 & 0,000 \\
\hline $\mathrm{GFI}_{\mathrm{MV}}^{\mathrm{MM}}$ & ] $0,90 ; 1]$ & 0,999 & 0,001 & 0,000 & 0,000 & 1,000 & 0,001 & 0,000 & 0,000 \\
\hline $\mathrm{AGFI}_{\mathrm{MV}}$ & ] $0,90 ; 1]$ & 0,983 & 0,017 & 0,051 & 0,220 & 0,990 & 0,010 & 0,003 & 0,055 \\
\hline $\mathrm{AGFI}_{\mathrm{MV}}^{\mathrm{GKO}}$ & ] $0,90 ; 1]$ & 0,729 & 0,211 & 0,947 & 0,224 & 0,748 & 0,144 & 0,983 & 0,129 \\
\hline $\mathrm{AGFI}_{\mathrm{MV}}^{\mathrm{EV}}$ & ] $0,90 ; 1]$ & 0,971 & 0,054 & 0,151 & 0,358 & 0,983 & 0,023 & 0,067 & 0,250 \\
\hline $\mathrm{AGFI}_{\mathrm{MV}}^{\mathrm{CDM}}$ & ] $0,90 ; 1]$ & 0,978 & 0,040 & 0,097 & 0,296 & 0,990 & 0,013 & 0,024 & 0,153 \\
\hline $\mathrm{AGFI}_{\mathrm{MV}}^{\mathrm{S}}$ & ] $0,90 ; 1]$ & 0,986 & 0,024 & 0,050 & 0,218 & 0,992 & 0,010 & 0,011 & 0,104 \\
\hline $\mathrm{AGFI}_{\mathrm{MV}}^{\mathrm{MM}}$ & ] $0,90 ; 1]$ & 0,997 & 0,005 & 0,001 & 0,032 & 0,998 & 0,003 & 0,000 & 0,000 \\
\hline
\end{tabular}


Tabela 5.15: Estatísticas dos mil valores obtidos via simulação para as estimativas dos parâmetros do MEE descrito pela Figura 5.2, por tamanho de amostra e com fração de 5\% de contaminação proveniente da lei Log-Normal Padrão.

\begin{tabular}{|c|c|c|c|c|c|}
\hline \multirow{3}{*}{$\begin{array}{c}\text { Parâmetro } \\
\lambda_{2,1}^{(\boldsymbol{x})}\end{array}$} & \multirow{3}{*}{$\begin{array}{c}\text { Referência } \\
\{44,0\}\end{array}$} & \multicolumn{2}{|c|}{$n=100$} & \multicolumn{2}{|c|}{$n=200$} \\
\hline & & $\hat{\mu}_{\hat{\theta}}$ & $\hat{\sigma}_{\hat{\theta}}$ & $\hat{\mu}_{\hat{\theta}}$ & $\hat{\sigma}_{\hat{\theta}}$ \\
\hline & & 3,922 & 0,405 & 3,894 & 0,276 \\
\hline$\lambda_{3,1}^{(\boldsymbol{x})}$ & $\{2,0\}$ & 1,979 & 0,224 & 1,967 & 0,150 \\
\hline$\lambda_{4,1}^{(\boldsymbol{x})}$ & $\{5,0\}$ & 4,771 & 0,498 & 4,779 & 0,341 \\
\hline$\phi_{1,1}$ & $\{1,0\}$ & 1,114 & 0,261 & 1,104 & 0,183 \\
\hline$\theta_{1,1}^{(\boldsymbol{\delta})}$ & $\{1,0\}$ & 0,997 & 0,145 & 1,041 & 0,101 \\
\hline$\theta_{2,2}^{(\boldsymbol{\delta})}$ & $\{1,0\}$ & 0,902 & 0,276 & 0,795 & 0,188 \\
\hline$\theta_{3,3}^{(\boldsymbol{\delta})}$ & $\{1,0\}$ & 1,033 & 0,157 & 1,065 & 0,110 \\
\hline \multirow[t]{2}{*}{$\theta_{4,4}^{(\boldsymbol{\delta})}$} & $\{1,0\}$ & 1,385 & 0,381 & 1,312 & 0,268 \\
\hline & & \multicolumn{2}{|c|}{$n=300$} & \multicolumn{2}{|c|}{$n=500$} \\
\hline \multirow{4}{*}{$\begin{array}{l}\text { Parâmetro } \\
\qquad \begin{array}{l}\lambda_{2,1}^{(\boldsymbol{x})} \\
\lambda_{3,1}^{(\boldsymbol{x})} \\
\lambda_{4,1}^{(\boldsymbol{x})}\end{array}\end{array}$} & \multirow{2}{*}{$\begin{array}{c}\text { Referência } \\
\quad\{4,0\}\end{array}$} & $\hat{\mu}_{\hat{\theta}}$ & $\hat{\sigma}_{\hat{\theta}}$ & $\hat{\mu}_{\hat{\theta}}$ & $\hat{\sigma}_{\hat{\theta}}$ \\
\hline & & 3,754 & 0,235 & 3,566 & 0,169 \\
\hline & $\{2,0\}$ & 1,887 & 0,124 & 1,853 & 0,089 \\
\hline & $\{5,0\}$ & 4,681 & 0,298 & 4,632 & 0,204 \\
\hline$\phi_{1,1}$ & $\{1,0\}$ & 1,167 & 0,171 & 1,240 & 0,129 \\
\hline$\theta_{1,1}^{(\boldsymbol{\delta})}$ & $\{1,0\}$ & 1,316 & 0,084 & 1,239 & 0,066 \\
\hline$\theta_{22}^{(\boldsymbol{\delta})}$ & $\{1,0\}$ & 0,924 & 0,153 & 1,395 & 0,118 \\
\hline$\theta_{3,3}^{(\boldsymbol{\delta})}$ & $\{1,0\}$ & 0,958 & 0,088 & 0,991 & 0,072 \\
\hline$\theta_{4,4}^{(\boldsymbol{\delta})}$ & $\{1,0\}$ & 1,046 & 0,214 & 0,640 & 0,179 \\
\hline
\end{tabular}


Tabela 5.16: Estatisticas dos mil valores obtidos via simulação para os indicadores clássicos e robustos do MEE descrito pela Figura 5.2, por tamanho de amostra e com fração de 5\% de contaminação proveniente da lei Log-Normal Padrão.

\begin{tabular}{|c|c|c|c|c|c|c|c|c|c|}
\hline \multirow{3}{*}{$\begin{array}{l}\text { Îndice } \\
\text { SRMR }\end{array}$} & \multirow{3}{*}{$\begin{array}{c}\text { Referência } \\
{[0 ; 0,08[}\end{array}$} & \multicolumn{4}{|c|}{$n=100$} & \multicolumn{4}{|c|}{$n=200$} \\
\hline & & $\hat{\mu}$ & $\hat{\sigma}$ & $\hat{\pi}$ & $\hat{\sigma}_{\hat{\pi}}$ & $\hat{\mu}$ & $\hat{\sigma}$ & $\hat{\pi}$ & $\hat{\sigma}_{\hat{\pi}}$ \\
\hline & & 0,010 & 0,005 & 0,000 & 0,000 & 0,010 & 0,005 & 0,000 & 0,000 \\
\hline RMSEA & {$[0 ; 0,08[$} & 0,046 & 0,059 & 0,277 & 0,448 & 0,050 & 0,050 & 0,282 & 0,450 \\
\hline NFI & ] $0,90 ; 1]$ & 0,994 & 0,005 & 0,000 & 0,000 & 0,996 & 0,003 & 0,000 & 0,000 \\
\hline IFI & ]0,90;1] & 0,999 & 0,005 & 0,000 & 0,000 & 0,998 & 0,003 & 0,000 & 0,000 \\
\hline NNFI & ] $0,90 ; 1]$ & 0,997 & 0,016 & 0,017 & 0,129 & 0,995 & 0,010 & 0,002 & 0,045 \\
\hline CFI & ] $0,90 ; 1]$ & 0,998 & 0,004 & 0,000 & 0,000 & 0,998 & 0,003 & 0,000 & 0,000 \\
\hline RNI & ]0,90;1] & 0,999 & 0,005 & 0,000 & 0,000 & 0,998 & 0,003 & 0,000 & 0,000 \\
\hline $\mathrm{GFI}_{\mathrm{MV}}$ & ] $0,90 ; 1]$ & 0,988 & 0,011 & 0,006 & 0,077 & 0,991 & 0,007 & 0,000 & 0,000 \\
\hline $\mathrm{GFI}_{\mathrm{MV}}^{\mathrm{GKO}}$ & ] $0,90 ; 1]$ & 0,905 & 0,120 & 0,534 & 0,499 & 0,935 & 0,060 & 0,469 & 0,499 \\
\hline $\mathrm{GFI}_{\mathrm{MV}}^{\mathrm{EV} M}$ & ] $0,90 ; 1]$ & 0,974 & 0,068 & 0,110 & 0,313 & 0,990 & 0,016 & 0,019 & 0,137 \\
\hline $\mathrm{GFI}_{\mathrm{MV}}^{\mathrm{CDM}}$ & ] $0,90 ; 1]$ & 0,975 & 0,067 & 0,109 & 0,312 & 0,992 & 0,016 & 0,011 & 0,104 \\
\hline $\mathrm{GFI}_{\mathrm{MV}}^{\mathrm{S}}$ & ] $0,90 ; 1]$ & 0,990 & 0,020 & 0,031 & 0,173 & 0,996 & 0,006 & 0,001 & 0,032 \\
\hline $\mathrm{GFI}_{\mathrm{MV}}^{\mathrm{MM}}$ & ] $0,90 ; 1]$ & 0,997 & 0,006 & 0,002 & 0,045 & 0,999 & 0,002 & 0,000 & 0,000 \\
\hline $\mathrm{AGFI}_{\mathrm{MV}}$ & ] $0,90 ; 1]$ & 0,939 & 0,053 & 0,471 & 0,499 & 0,957 & 0,037 & 0,335 & 0,472 \\
\hline $\mathrm{AGFI}_{\mathrm{MV}}^{\mathrm{GKO}}$ & ] $0,90 ; 1]$ & 0,523 & 0,602 & 0,869 & 0,338 & 0,674 & 0,302 & 0,929 & 0,257 \\
\hline $\mathrm{AGFI}_{\mathrm{MV}}^{\mathrm{EVM}}$ & ] $0,90 ; 1]$ & 0,868 & 0,339 & 0,533 & 0,499 & 0,949 & 0,080 & 0,318 & 0,466 \\
\hline $\mathrm{AGFI}_{\mathrm{MV}}^{\mathrm{CDM}}$ & ] $0,90 ; 1]$ & 0,875 & 0,337 & 0,534 & 0,499 & 0,958 & 0,078 & 0,257 & 0,437 \\
\hline $\mathrm{AGFI}_{\mathrm{MV}}^{\mathrm{S}}$ & ] $0,90 ; 1]$ & 0,948 & 0,100 & 0,285 & 0,452 & 0,978 & 0,030 & 0,115 & 0,319 \\
\hline \multirow[t]{2}{*}{$\mathrm{AGFI}_{\mathrm{MV}}^{\mathrm{MM}}$} & ] $0,90 ; 1]$ & 0,984 & 0,030 & 0,071 & 0,257 & 0,995 & 0,010 & 0,009 & 0,094 \\
\hline & & \multicolumn{4}{|c|}{$n=300$} & \multicolumn{4}{|c|}{$n=500$} \\
\hline Índice & Referência & $\hat{\mu}$ & $\hat{\sigma}$ & $\hat{\pi}$ & $\hat{\sigma}_{\hat{\pi}}$ & $\hat{\mu}$ & $\hat{\sigma}$ & $\hat{\pi}$ & $\hat{\sigma}_{\hat{\pi}}$ \\
\hline SRMR & {$[0 ; 0,08[$} & 0,007 & 0,004 & 0,000 & 0,000 & 0,010 & 0,003 & 0,000 & 0,000 \\
\hline RMSEA & {$[0 ; 0,08[$} & 0,030 & 0,036 & 0,105 & 0,307 & 0,097 & 0,032 & 0,716 & 0,451 \\
\hline NFI & ] $0,90 ; 1]$ & 0,998 & 0,002 & 0,000 & 0,000 & 0,995 & 0,003 & 0,000 & 0,000 \\
\hline IFI & ] $0,90 ; 1]$ & 0,999 & 0,002 & 0,000 & 0,000 & 0,995 & 0,003 & 0,000 & 0,000 \\
\hline NNFI & $0,90 ; 1]$ & 0,998 & 0,006 & 0,000 & 0,000 & 0,986 & 0,008 & 0,001 & 0,032 \\
\hline CFI & ] $0,90 ; 1]$ & 0,999 & 0,002 & 0,000 & 0,000 & 0,995 & 0,003 & 0,000 & 0,000 \\
\hline RNI & ] $0,90 ; 1]$ & 0,999 & 0,002 & 0,000 & 0,000 & 0,995 & 0,003 & 0,000 & 0,000 \\
\hline $\mathrm{GFI}_{\mathrm{MV}}$ & ] $0,90 ; 1]$ & 0,996 & 0,004 & 0,000 & 0,000 & 0,988 & 0,005 & 0,000 & 0,000 \\
\hline $\mathrm{GFI}_{\mathrm{MV}}^{\mathrm{GKO}}$ & ] $0,90 ; 1]$ & 0,940 & 0,047 & 0,467 & 0,499 & 0,934 & 0,036 & 0,605 & 0,489 \\
\hline $\mathrm{GFI}_{\mathrm{MV}}^{\mathrm{EVM}}$ & ] $0,90 ; 1]$ & 0,994 & 0,009 & 0,005 & 0,071 & 0,996 & 0,006 & 0,003 & 0,055 \\
\hline $\mathrm{GFI}_{\mathrm{MV}}^{\mathrm{CDM}}$ & ] $0,90 ; 1]$ & 0,995 & 0,006 & 0,000 & 0,000 & 0,997 & 0,003 & 0,000 & 0,000 \\
\hline $\mathrm{GFI}_{\mathrm{MV}}^{\mathrm{S}}$ & ] $0,90 ; 1]$ & 0,997 & 0,004 & 0,000 & 0,000 & 0,997 & 0,003 & 0,000 & 0,000 \\
\hline $\mathrm{GFI}_{\mathrm{MV}}^{\mathrm{MM}}$ & ] $0,90 ; 1]$ & 0,999 & 0,001 & 0,000 & 0,000 & 0,998 & 0,002 & 0,000 & 0,000 \\
\hline $\mathrm{AGFI}_{\mathrm{MV}}$ & ] $0,90 ; 1]$ & 0,978 & 0,021 & 0,093 & 0,291 & 0,942 & 0,027 & 0,594 & 0,491 \\
\hline $\mathrm{AGFI}_{\mathrm{MV}}^{\mathrm{GKO}}$ & ] $0,90 ; 1]$ & 0,702 & 0,233 & 0,945 & 0,228 & 0,670 & 0,179 & 0,994 & 0,077 \\
\hline $\mathrm{AGFI}_{\mathrm{MV}}^{\mathrm{EVM}}$ & ] $0,90 ; 1]$ & 0,968 & 0,044 & 0,199 & 0,399 & 0,980 & 0,031 & 0,084 & 0,278 \\
\hline $\mathrm{AGFI}_{\mathrm{MV}}^{\mathrm{CDM}}$ & ] $0,90 ; 1]$ & 0,974 & 0,031 & 0,141 & 0,348 & 0,987 & 0,017 & 0,032 & 0,176 \\
\hline $\mathrm{AGFI}_{\mathrm{MV}}^{\mathrm{S}}$ & ] $0,90 ; 1]$ & 0,984 & 0,021 & 0,066 & 0,248 & 0,987 & 0,015 & 0,032 & 0,176 \\
\hline $\mathrm{AGFI}_{\mathrm{MV}}^{\mathrm{MM}}$ & ] $0,90 ; 1]$ & 0,995 & 0,007 & 0,006 & 0,077 & 0,991 & 0,008 & 0,004 & 0,063 \\
\hline
\end{tabular}


Tabela 5.17: Estatísticas dos mil valores obtidos via simulação para as estimativas dos parâmetros do MEE descrito pela Figura 5.2, por tamanho de amostra e com fração de $25 \%$ de contaminação proveniente da lei Log-Normal Padrão.

\begin{tabular}{|c|c|c|c|c|c|}
\hline \multirow{3}{*}{$\begin{array}{c}\text { Parâmetro } \\
\lambda_{2,1}^{(\boldsymbol{x})}\end{array}$} & \multirow{3}{*}{$\begin{array}{c}\text { Referência } \\
\qquad\{4,0\}\end{array}$} & \multicolumn{2}{|c|}{$n=100$} & \multicolumn{2}{|c|}{$n=200$} \\
\hline & & $\hat{\mu}_{\hat{\theta}}$ & $\hat{\sigma}_{\hat{\theta}}$ & $\hat{\mu}_{\hat{\theta}}$ & $\hat{\sigma}_{\hat{\theta}}$ \\
\hline & & 2,541 & 0,249 & 3,106 & 0,216 \\
\hline$\lambda_{3,1}^{(\boldsymbol{x})}$ & $\{2,0\}$ & 1,486 & 0,124 & 1,849 & 0,108 \\
\hline$\lambda_{4,1}^{(\boldsymbol{x})}$ & $\{5,0\}$ & 3,627 & 0,295 & 4,011 & 0,270 \\
\hline$\phi_{1,1}$ & $\{1,0\}$ & 2,404 & 0,491 & 1,747 & 0,284 \\
\hline$\theta_{1,1}^{(\boldsymbol{\delta})}$ & $\{1,0\}$ & 1,883 & 0,181 & 1,473 & 0,107 \\
\hline$\theta_{2,2}^{(\boldsymbol{\delta})}$ & $\{1,0\}$ & 2,087 & 0,240 & 1,225 & 0,133 \\
\hline$\theta_{3,3}^{(\boldsymbol{\delta})}$ & $\{1,0\}$ & 0,871 & 0,149 & 1,951 & 0,133 \\
\hline \multirow[t]{2}{*}{$\theta_{4,4}^{(\boldsymbol{\delta})}$} & $\{1,0\}$ & 0,298 & 0,240 & 0,493 & 0,199 \\
\hline & & \multicolumn{2}{|c|}{$n=300$} & \multicolumn{2}{|c|}{$n=500$} \\
\hline \multirow{4}{*}{$\begin{array}{l}\text { Parâmetro } \\
\qquad \begin{array}{l}\lambda_{2,1}^{(\boldsymbol{x})} \\
\lambda_{3,1}^{(\boldsymbol{x})} \\
\lambda_{4,1}^{(\boldsymbol{x})}\end{array}\end{array}$} & \multirow{2}{*}{$\begin{array}{c}\text { Referência } \\
\quad\{4,0\}\end{array}$} & $\hat{\mu}_{\hat{\theta}}$ & $\hat{\sigma}_{\hat{\theta}}$ & $\hat{\mu}_{\hat{\theta}}$ & $\hat{\sigma}_{\hat{\theta}}$ \\
\hline & & 3,264 & 0,147 & 3,082 & 0,116 \\
\hline & $\{2,0\}$ & 1,793 & 0,074 & 1,610 & 0,059 \\
\hline & $\{5,0\}$ & 3,583 & 0,178 & 3,633 & 0,146 \\
\hline$\phi_{1,1}$ & $\{1,0\}$ & 1,853 & 0,212 & 2,011 & 0,194 \\
\hline$\theta_{1,1}^{(\boldsymbol{\delta})}$ & $\{1,0\}$ & 1,311 & 0,084 & 1,566 & 0,073 \\
\hline$\theta_{22}^{(\boldsymbol{\delta})}$ & $\{1,0\}$ & 0,082 & 0,073 & 0,477 & 0,102 \\
\hline$\theta_{3,3}^{(\boldsymbol{\delta})}$ & $\{1,0\}$ & 1,442 & 0,096 & 0,956 & 0,067 \\
\hline$\theta_{4,4}^{(\boldsymbol{\delta})}$ & $\{1,0\}$ & 2,901 & 0,199 & 1,540 & 0,138 \\
\hline
\end{tabular}


Tabela 5.18: Estatisticas dos mil valores obtidos via simulação para os indicadores clássicos e robustos do MEE descrito pela Figura 5.2, por tamanho de amostra e com fração de $25 \%$ de contaminação proveniente da lei Log-Normal Padrão.

\begin{tabular}{|c|c|c|c|c|c|c|c|c|c|}
\hline \multirow{3}{*}{$\begin{array}{l}\text { Índice } \\
\text { SRMR }\end{array}$} & \multirow{3}{*}{$\begin{array}{c}\text { Referência } \\
{[0 ; 0,08[}\end{array}$} & \multicolumn{4}{|c|}{$n=100$} & \multicolumn{4}{|c|}{$n=200$} \\
\hline & & $\hat{\mu}$ & $\hat{\sigma}$ & $\hat{\pi}$ & $\hat{\sigma}_{\hat{\pi}}$ & $\hat{\mu}$ & $\hat{\sigma}$ & $\hat{\pi}$ & $\hat{\sigma}_{\hat{\pi}}$ \\
\hline & & 0,031 & 0,007 & 0,000 & 0,000 & 0,054 & 0,008 & 0,002 & 0,045 \\
\hline RMSEA & {$[0 ; 0,08[$} & 0,317 & 0,060 & 0,999 & 0,032 & 0,386 & 0,039 & 1,000 & 0,000 \\
\hline NFI & ] $0,90 ; 1]$ & 0,954 & 0,015 & 0,391 & 0,488 & 0,936 & 0,012 & 0,868 & 0,339 \\
\hline IFI & ] $0,90 ; 1]$ & 0,958 & 0,015 & 0,293 & 0,455 & 0,938 & 0,012 & 0,829 & 0,377 \\
\hline NNFI & ] $0,90 ; 1]$ & 0,872 & 0,047 & 0,965 & 0,184 & 0,814 & 0,037 & 1,000 & 0,000 \\
\hline CFI & ] $0,90 ; 1]$ & 0,957 & 0,016 & 0,302 & 0,459 & 0,938 & 0,012 & 0,835 & 0,371 \\
\hline RNI & ]0,90;1] & 0,957 & 0,016 & 0,302 & 0,459 & 0,938 & 0,012 & 0,835 & 0,371 \\
\hline $\mathrm{GFI}_{\mathrm{MV}}$ & ] $0,90 ; 1]$ & 0,914 & 0,022 & 0,941 & 0,236 & 0,879 & 0,018 & 1,000 & 0,000 \\
\hline $\mathrm{GFI}_{\mathrm{MV}}^{\mathrm{GKO}}$ & ] $0,90 ; 1]$ & 0,791 & 0,212 & 0,864 & 0,343 & 0,862 & 0,104 & 0,841 & 0,366 \\
\hline $\mathrm{GFI}_{\mathrm{MV}}^{\mathrm{EVM}}$ & ] $0,90 ; 1]$ & 0,962 & 0,047 & 0,223 & 0,416 & 0,980 & 0,018 & 0,062 & 0,241 \\
\hline $\mathrm{GFI}_{\mathrm{MV}}^{\mathrm{CDM}}$ & ] $0,90 ; 1]$ & 0,950 & 0,043 & 0,407 & 0,492 & 0,949 & 0,032 & 0,452 & 0,498 \\
\hline $\mathrm{GFI}_{\mathrm{MV}}^{\mathrm{S}}$ & ] $0,90 ; 1]$ & 0,974 & 0,035 & 0,092 & 0,289 & 0,987 & 0,010 & 0,011 & 0,104 \\
\hline $\mathrm{GFI}_{\mathrm{MV}}^{\mathrm{MM}}$ & ] $0,90 ; 1]$ & 0,961 & 0,037 & 0,244 & 0,430 & 0,976 & 0,020 & 0,090 & 0,286 \\
\hline $\mathrm{AGFI}_{\mathrm{MV}}$ & ] $0,90 ; 1]$ & 0,570 & 0,112 & 1,000 & 0,000 & 0,395 & 0,089 & 1,000 & 0,000 \\
\hline $\mathrm{AGFI}_{\mathrm{MV}}^{\mathrm{GKO}}$ & ] $0,90 ; 1]$ & $-0,045$ & 1,062 & 0,992 & 0,089 & 0,309 & 0,520 & 0,994 & 0,077 \\
\hline $\mathrm{AGFI}_{\mathrm{MV}}^{\mathrm{EVM}}$ & ] $0,90 ; 1]$ & 0,810 & 0,236 & 0,881 & 0,324 & 0,902 & 0,090 & 0,668 & 0,471 \\
\hline $\mathrm{AGFI}_{\mathrm{MV}}^{\mathrm{CDM}}$ & ] $0,90 ; 1]$ & 0,749 & 0,217 & 0,935 & 0,247 & 0,745 & 0,160 & 0,949 & 0,220 \\
\hline $\mathrm{AGFI}_{\mathrm{MV}}^{\mathrm{S}}$ & ] $0,90 ; 1]$ & 0,872 & 0,173 & 0,807 & 0,395 & 0,935 & 0,051 & 0,496 & 0,500 \\
\hline \multirow[t]{2}{*}{$\mathrm{AGFI}_{\mathrm{MV}}^{\mathrm{MM}}$} & ] $0,90 ; 1]$ & 0,807 & 0,187 & 0,841 & 0,366 & 0,878 & 0,098 & 0,783 & 0,412 \\
\hline & & \multicolumn{4}{|c|}{$n=300$} & \multicolumn{4}{|c|}{$n=500$} \\
\hline Índice & Referência & $\hat{\mu}$ & $\hat{\sigma}$ & $\hat{\pi}$ & $\hat{\sigma}_{\hat{\pi}}$ & $\hat{\mu}$ & $\hat{\sigma}$ & $\hat{\pi}$ & $\hat{\sigma}_{\hat{\pi}}$ \\
\hline SRMR & {$[0 ; 0,08[$} & 0,042 & 0,006 & 0,000 & 0,000 & 0,024 & 0,003 & 0,000 & 0,000 \\
\hline RMSEA & {$[0 ; 0,08[$} & 0,350 & 0,035 & 1,000 & 0,000 & 0,251 & 0,029 & 1,000 & 0,000 \\
\hline NFI & ] $0,90 ; 1]$ & 0,948 & 0,010 & 0,564 & 0,496 & 0,975 & 0,006 & 0,000 & 0,000 \\
\hline IFI & ] $0,90 ; 1]$ & 0,949 & 0,010 & 0,507 & 0,500 & 0,976 & 0,006 & 0,000 & 0,000 \\
\hline NNFI & ] $0,90 ; 1]$ & 0,848 & 0,031 & 1,000 & 0,000 & 0,927 & 0,017 & 0,920 & 0,271 \\
\hline CFI & ] $0,90 ; 1]$ & 0,949 & 0,010 & 0,511 & 0,500 & 0,976 & 0,006 & 0,000 & 0,000 \\
\hline RNI & ] $0,90 ; 1]$ & 0,949 & 0,010 & 0,511 & 0,500 & 0,976 & 0,006 & 0,000 & 0,000 \\
\hline $\mathrm{GFI}_{\mathrm{MV}}$ & ]0,90;1] & 0,895 & 0,016 & 1,000 & 0,000 & 0,944 & 0,011 & 0,705 & 0,456 \\
\hline $\mathrm{GFI}_{\mathrm{MV}}^{\mathrm{GKO}}$ & ] $0,90 ; 1]$ & 0,888 & 0,070 & 0,855 & 0,352 & 0,891 & 0,052 & 0,912 & 0,283 \\
\hline $\mathrm{GFI}_{\mathrm{MV}}^{\mathrm{EVM}}$ & ] $0,90 ; 1]$ & 0,985 & 0,013 & 0,024 & 0,153 & 0,992 & 0,007 & 0,002 & 0,045 \\
\hline $\mathrm{GFI}_{\mathrm{MV}}^{\mathrm{CDM}}$ & ] $0,90 ; 1]$ & 0,960 & 0,023 & 0,278 & 0,448 & 0,961 & 0,020 & 0,264 & 0,441 \\
\hline $\mathrm{GFI}_{\mathrm{MV}}^{\mathrm{S}}$ & ] $0,90 ; 1]$ & 0,990 & 0,006 & 0,001 & 0,032 & 0,994 & 0,003 & 0,000 & 0,000 \\
\hline $\mathrm{GFI}_{\mathrm{MV}}^{\mathrm{MM}}$ & ] $0,90 ; 1]$ & 0,984 & 0,011 & 0,020 & 0,140 & 0,983 & 0,009 & 0,007 & 0,083 \\
\hline $\mathrm{AGFI}_{\mathrm{MV}}$ & ] $0,90 ; 1]$ & 0,473 & 0,082 & 1,000 & 0,000 & 0,720 & 0,054 & 1,000 & 0,000 \\
\hline $\mathrm{AGFI}_{\mathrm{MV}}^{\mathrm{GKO}}$ & ] $0,90 ; 1]$ & 0,441 & 0,350 & 1,000 & 0,000 & 0,456 & 0,262 & 1,000 & 0,000 \\
\hline $\mathrm{AGFI}_{\mathrm{MV}}^{\mathrm{EVM}}$ & ] $0,90 ; 1]$ & 0,925 & 0,066 & 0,548 & 0,498 & 0,958 & 0,034 & 0,254 & 0,436 \\
\hline $\mathrm{AGFI}_{\mathrm{MV}}^{\mathrm{CDM}}$ & ] $0,90 ; 1]$ & 0,801 & 0,117 & 0,952 & 0,214 & 0,804 & 0,102 & 0,953 & 0,212 \\
\hline $\mathrm{AGFI}_{\mathrm{MV}}^{\mathrm{S}}$ & ] $0,90 ; 1]$ & 0,949 & 0,030 & 0,374 & 0,484 & 0,969 & 0,017 & 0,109 & 0,312 \\
\hline $\mathrm{AGFI}_{\mathrm{MV}}^{\mathrm{MM}}$ & ] $0,90 ; 1]$ & 0,918 & 0,057 & 0,674 & 0,469 & 0,917 & 0,045 & 0,765 & 0,424 \\
\hline
\end{tabular}


Tabela 5.19: Estatísticas dos mil valores obtidos via simulação para as estimativas dos parâmetros do MEE descrito pela Figura 5.2, por tamanho de amostra e com fração de 5\% de contaminação proveniente da lei Uniforme Continua em $]-5 ; 5 /$.

\begin{tabular}{|c|c|c|c|c|c|}
\hline \multirow{3}{*}{$\begin{array}{c}\text { Parâmetro } \\
\lambda_{2,1}^{(\boldsymbol{x})}\end{array}$} & \multirow{3}{*}{$\begin{array}{c}\text { Referência } \\
\{44,0\}\end{array}$} & \multicolumn{2}{|c|}{$n=100$} & \multicolumn{2}{|c|}{$n=200$} \\
\hline & & $\hat{\mu}_{\hat{\theta}}$ & $\hat{\sigma}_{\hat{\theta}}$ & $\hat{\mu}_{\hat{\theta}}$ & $\hat{\sigma}_{\hat{\theta}}$ \\
\hline & & 3,794 & 0,392 & 3,773 & 0,291 \\
\hline$\lambda_{3,1}^{(\boldsymbol{x})}$ & $\{2,0\}$ & 1,952 & 0,222 & 1,942 & 0,156 \\
\hline$\lambda_{4,1}^{(\boldsymbol{x})}$ & $\{5,0\}$ & 4,740 & 0,505 & 4,803 & 0,366 \\
\hline$\phi_{1,1}$ & $\{1,0\}$ & 1,157 & 0,277 & 1,135 & 0,198 \\
\hline$\theta_{1,1}^{(\boldsymbol{\delta})}$ & $\{1,0\}$ & 1,157 & 0,142 & 1,181 & 0,100 \\
\hline$\theta_{2,2}^{(\boldsymbol{\delta})}$ & $\{1,0\}$ & 1,156 & 0,247 & 1,283 & 0,182 \\
\hline$\theta_{3,3}^{(\boldsymbol{\delta})}$ & $\{1,0\}$ & 1,195 & 0,154 & 1,147 & 0,111 \\
\hline \multirow[t]{2}{*}{$\theta_{4,4}^{(\boldsymbol{\delta})}$} & $\{1,0\}$ & 1,040 & 0,366 & 0,688 & 0,265 \\
\hline & & \multicolumn{2}{|c|}{$n=300$} & \multicolumn{2}{|c|}{$n=500$} \\
\hline \multirow{4}{*}{$\begin{array}{l}\text { Parâmetro } \\
\qquad \begin{array}{l}\lambda_{2,1}^{(\boldsymbol{x})} \\
\lambda_{3,1}^{(\boldsymbol{x})} \\
\lambda_{4,1}^{(\boldsymbol{x})}\end{array}\end{array}$} & \multirow{2}{*}{$\begin{array}{c}\text { Referência } \\
\quad\{4,0\}\end{array}$} & $\hat{\mu}_{\hat{\theta}}$ & $\hat{\sigma}_{\hat{\theta}}$ & $\hat{\mu}_{\hat{\theta}}$ & $\hat{\sigma}_{\hat{\theta}}$ \\
\hline & & 3,875 & 0,257 & 3,734 & 0,173 \\
\hline & $\{2,0\}$ & 1,961 & 0,138 & 1,906 & 0,092 \\
\hline & $\{5,0\}$ & 4,715 & 0,318 & 4,600 & 0,214 \\
\hline$\phi_{1,1}$ & $\{1,0\}$ & 1,129 & 0,173 & 1,199 & 0,134 \\
\hline$\theta_{1,1}^{(\boldsymbol{\delta})}$ & $\{1,0\}$ & 1,261 & 0,084 & 1,310 & 0,063 \\
\hline$\theta_{22}^{(\boldsymbol{\delta})}$ & $\{1,0\}$ & 0,715 & 0,159 & 0,838 & 0,115 \\
\hline$\theta_{3,3}^{(\boldsymbol{\delta})}$ & $\{1,0\}$ & 1,157 & 0,087 & 1,100 & 0,070 \\
\hline$\theta_{4,4}^{(\boldsymbol{\delta})}$ & $\{1,0\}$ & 1,485 & 0,217 & 1,256 & 0,167 \\
\hline
\end{tabular}


Tabela 5.20: Estatísticas dos mil valores obtidos via simulação para os indicadores clássicos e robustos do MEE descrito pela Figura 5.2, por tamanho de amostra e com fração de 5\% de contaminação proveniente da lei Uniforme Continua em ]-5;5/.

\begin{tabular}{|c|c|c|c|c|c|c|c|c|c|}
\hline \multirow{3}{*}{$\begin{array}{l}\text { Îndice } \\
\text { SRMR }\end{array}$} & \multirow{3}{*}{$\begin{array}{c}\text { Referência } \\
{[0 ; 0,08[}\end{array}$} & \multicolumn{4}{|c|}{$n=100$} & \multicolumn{4}{|c|}{$n=200$} \\
\hline & & $\hat{\mu}$ & $\hat{\sigma}$ & $\hat{\pi}$ & $\hat{\sigma}_{\hat{\pi}}$ & $\hat{\mu}$ & $\hat{\sigma}$ & $\hat{\pi}$ & $\hat{\sigma}_{\hat{\pi}}$ \\
\hline & & 0,014 & 0,007 & 0,000 & 0,000 & 0,008 & 0,004 & 0,000 & 0,000 \\
\hline RMSEA & {$[0 ; 0,08[$} & 0,087 & 0,073 & 0,542 & 0,498 & 0,032 & 0,042 & 0,164 & 0,370 \\
\hline NFI & ] $0,90 ; 1]$ & 0,990 & 0,008 & 0,000 & 0,000 & 0,997 & 0,003 & 0,000 & 0,000 \\
\hline IFI & ] $0,90 ; 1]$ & 0,995 & 0,008 & 0,000 & 0,000 & 0,999 & 0,003 & 0,000 & 0,000 \\
\hline NNFI & ] $0,90 ; 1]$ & 0,984 & 0,023 & 0,084 & 0,278 & 0,998 & 0,008 & 0,001 & 0,032 \\
\hline CFI & ] $0,90 ; 1]$ & 0,994 & 0,007 & 0,000 & 0,000 & 0,999 & 0,002 & 0,000 & 0,000 \\
\hline RNI & ]0,90;1] & 0,995 & 0,008 & 0,000 & 0,000 & 0,999 & 0,003 & 0,000 & 0,000 \\
\hline $\mathrm{GFI}_{\mathrm{MV}}$ & ] $0,90 ; 1]$ & 0,981 & 0,014 & 0,031 & 0,173 & 0,994 & 0,005 & 0,000 & 0,000 \\
\hline $\mathrm{GFI}_{\mathrm{MV}}^{\mathrm{GKO}}$ & ] $0,90 ; 1]$ & 0,900 & 0,136 & 0,548 & 0,498 & 0,929 & 0,064 & 0,521 & 0,500 \\
\hline $\mathrm{GFI}_{\mathrm{MV}}^{\mathrm{EVM}}$ & ] $0,90 ; 1]$ & 0,972 & 0,070 & 0,125 & 0,331 & 0,990 & 0,014 & 0,016 & 0,126 \\
\hline $\mathrm{GFI}_{\mathrm{MV}}^{\mathrm{CDM}}$ & ] $0,90 ; 1]$ & 0,977 & 0,044 & 0,102 & 0,303 & 0,992 & 0,012 & 0,009 & 0,094 \\
\hline $\mathrm{GFI}_{\mathrm{MV}}^{\mathrm{S}}$ & ] $0,90 ; 1]$ & 0,989 & 0,019 & 0,026 & 0,159 & 0,995 & 0,007 & 0,004 & 0,063 \\
\hline $\mathrm{GFI}_{\mathrm{MV}}^{\mathrm{MM}}$ & ] $0,90 ; 1]$ & 0,997 & 0,005 & 0,002 & 0,045 & 0,999 & 0,002 & 0,000 & 0,000 \\
\hline $\mathrm{AGFI}_{\mathrm{MV}}$ & ] $0,90 ; 1]$ & 0,903 & 0,069 & 0,703 & 0,457 & 0,970 & 0,027 & 0,196 & 0,397 \\
\hline $\mathrm{AGFI}_{\mathrm{MV}}^{\mathrm{GKO}}$ & ] $0,90 ; 1]$ & 0,500 & 0,682 & 0,878 & 0,327 & 0,647 & 0,321 & 0,935 & 0,247 \\
\hline $\mathrm{AGFI}_{\mathrm{MV}}^{\mathrm{EVM}}$ & ] $0,90 ; 1]$ & 0,861 & 0,349 & 0,591 & 0,492 & 0,952 & 0,068 & 0,319 & 0,466 \\
\hline $\mathrm{AGFI}_{\mathrm{MV}}^{\mathrm{CDM}}$ & ] $0,90 ; 1]$ & 0,883 & 0,220 & 0,580 & 0,494 & 0,959 & 0,059 & 0,251 & 0,434 \\
\hline $\mathrm{AGFI}_{\mathrm{MV}}^{\mathrm{S}}$ & ] $0,90 ; 1]$ & 0,945 & 0,097 & 0,325 & 0,469 & 0,976 & 0,034 & 0,126 & 0,332 \\
\hline \multirow[t]{2}{*}{$\mathrm{AGFI}_{\mathrm{MV}}^{\mathrm{MM}}$} & ] $0,90 ; 1]$ & 0,986 & 0,027 & 0,064 & 0,245 & 0,993 & 0,012 & 0,012 & 0,109 \\
\hline & & \multicolumn{4}{|c|}{$n=300$} & \multicolumn{4}{|c|}{$n=500$} \\
\hline Índice & Referência & $\hat{\mu}$ & $\hat{\sigma}$ & $\hat{\pi}$ & $\hat{\sigma}_{\hat{\pi}}$ & $\hat{\mu}$ & $\hat{\sigma}$ & $\hat{\pi}$ & $\hat{\sigma}_{\hat{\pi}}$ \\
\hline SRMR & {$[0 ; 0,08[$} & 0,005 & 0,003 & 0,000 & 0,000 & 0,011 & 0,004 & 0,000 & 0,000 \\
\hline RMSEA & {$[0 ; 0,08[$} & 0,013 & 0,024 & 0,025 & 0,156 & 0,065 & 0,031 & 0,336 & 0,473 \\
\hline NFI & ]0,90;1] & 0,999 & 0,001 & 0,000 & 0,000 & 0,997 & 0,002 & 0,000 & 0,000 \\
\hline IFI & ] $0,90 ; 1]$ & 1,000 & 0,001 & 0,000 & 0,000 & 0,998 & 0,002 & 0,000 & 0,000 \\
\hline NNFI & ] $0,90 ; 1]$ & 1,001 & 0,003 & 0,000 & 0,000 & 0,993 & 0,006 & 0,000 & 0,000 \\
\hline CFI & ] $0,90 ; 1]$ & 1,000 & 0,001 & 0,000 & 0,000 & 0,998 & 0,002 & 0,000 & 0,000 \\
\hline RNI & ] $0,90 ; 1]$ & 1,000 & 0,001 & 0,000 & 0,000 & 0,998 & 0,002 & 0,000 & 0,000 \\
\hline $\mathrm{GFI}_{\mathrm{MV}}$ & ]0,90;1] & 0,997 & 0,002 & 0,000 & 0,000 & 0,993 & 0,004 & 0,000 & 0,000 \\
\hline $\mathrm{GFI}_{\mathrm{MV}}^{\mathrm{GKO}}$ & ] $0,90 ; 1]$ & 0,935 & 0,046 & 0,543 & 0,498 & 0,938 & 0,034 & 0,545 & 0,498 \\
\hline $\mathrm{GFI}_{\mathrm{MV}}^{\mathrm{EVM}}$ & ] $0,90 ; 1]$ & 0,993 & 0,009 & 0,003 & 0,055 & 0,996 & 0,005 & 0,000 & 0,000 \\
\hline $\mathrm{GFI}_{\mathrm{MV}}^{\mathrm{CDM}}$ & ] $0,90 ; 1]$ & 0,995 & 0,006 & 0,000 & 0,000 & 0,997 & 0,003 & 0,000 & 0,000 \\
\hline $\mathrm{GFI}_{\mathrm{MV}}^{\mathrm{S}}$ & ] $0,90 ; 1]$ & 0,997 & 0,004 & 0,000 & 0,000 & 0,998 & 0,002 & 0,000 & 0,000 \\
\hline $\mathrm{GFI}_{\mathrm{MV}}^{\mathrm{MM}}$ & ] $0,90 ; 1]$ & 0,999 & 0,002 & 0,000 & 0,000 & 0,999 & 0,001 & 0,000 & 0,000 \\
\hline $\mathrm{AGFI}_{\mathrm{MV}}$ & ] $0,90 ; 1]$ & 0,987 & 0,012 & 0,020 & 0,140 & 0,965 & 0,020 & 0,214 & 0,410 \\
\hline $\mathrm{AGFI}_{\mathrm{MV}}^{\mathrm{GKO}}$ & ] $0,90 ; 1]$ & 0,675 & 0,232 & 0,971 & 0,168 & 0,691 & 0,170 & 0,992 & 0,089 \\
\hline $\mathrm{AGFI}_{\mathrm{MV}}^{\mathrm{EVM}}$ & ] $0,90 ; 1]$ & 0,964 & 0,047 & 0,233 & 0,423 & 0,979 & 0,024 & 0,083 & 0,276 \\
\hline $\mathrm{AGFI}_{\mathrm{MV}}^{\mathrm{CDM}}$ & ] $0,90 ; 1]$ & 0,974 & 0,029 & 0,156 & 0,363 & 0,986 & 0,015 & 0,027 & 0,162 \\
\hline $\mathrm{AGFI}_{\mathrm{MV}}^{\mathrm{S}}$ & ] $0,90 ; 1]$ & 0,983 & 0,019 & 0,060 & 0,238 & 0,989 & 0,011 & 0,009 & 0,094 \\
\hline $\mathrm{AGFI}_{\mathrm{MV}}^{\mathrm{MM}}$ & ] $0,90 ; 1]$ & 0,995 & 0,008 & 0,003 & 0,055 & 0,995 & 0,006 & 0,000 & 0,000 \\
\hline
\end{tabular}


Tabela 5.21: Estatísticas dos mil valores obtidos via simulação para as estimativas dos parâmetros do MEE descrito pela Figura 5.2, por tamanho de amostra e com fração de $25 \%$ de contaminação proveniente da lei Uniforme Continua em ]-5; 5/.

\begin{tabular}{|c|c|c|c|c|c|}
\hline \multirow{3}{*}{$\begin{array}{c}\text { Parâmetro } \\
\lambda_{2,1}^{(\boldsymbol{x})}\end{array}$} & \multirow{3}{*}{$\begin{array}{c}\text { Referência } \\
\quad\{4,0\}\end{array}$} & \multicolumn{2}{|c|}{$n=100$} & \multicolumn{2}{|c|}{$n=200$} \\
\hline & & $\hat{\mu}_{\hat{\theta}}$ & $\hat{\sigma}_{\hat{\theta}}$ & $\hat{\mu}_{\hat{\theta}}$ & $\hat{\sigma}_{\hat{\theta}}$ \\
\hline & & 3,003 & 0,334 & 3,114 & 0,200 \\
\hline$\lambda_{3,1}^{(\boldsymbol{x})}$ & $\{2,0\}$ & 1,638 & 0,173 & 1,730 & 0,097 \\
\hline$\lambda_{4,1}^{(x)}$ & $\{5,0\}$ & 3,555 & 0,401 & 3,815 & 0,245 \\
\hline$\phi_{1,1}$ & $\{1,0\}$ & 2,116 & 0,512 & 1,903 & 0,290 \\
\hline$\theta_{1,1}^{(\boldsymbol{\delta})}$ & $\{1,0\}$ & 2,402 & 0,179 & 1,662 & 0,108 \\
\hline$\theta_{2,2}^{(\boldsymbol{\delta})}$ & $\{1,0\}$ & 0,387 & 0,196 & 0,683 & 0,128 \\
\hline$\theta_{3,3}^{(\boldsymbol{\delta})}$ & $\{1,0\}$ & 1,452 & 0,160 & 1,410 & 0,113 \\
\hline$\theta_{4,4}^{(\boldsymbol{\delta})}$ & $\{1,0\}$ & 1,768 & 0,307 & 1,085 & 0,178 \\
\hline \multirow{5}{*}{$\begin{array}{l}\text { Parâmetro } \\
\qquad \begin{array}{c}\lambda_{2,1}^{(\boldsymbol{x})} \\
\lambda_{3,1}^{(\boldsymbol{x})} \\
\lambda_{4,1}^{(\boldsymbol{x})}\end{array}\end{array}$} & \multirow{3}{*}{$\begin{array}{c}\text { Referência } \\
\qquad\{4,0\}\end{array}$} & \multicolumn{2}{|c|}{$n=300$} & \multicolumn{2}{|c|}{$n=500$} \\
\hline & & $\hat{\mu}_{\hat{\theta}}$ & $\hat{\sigma}_{\hat{\theta}}$ & $\hat{\mu}_{\hat{\theta}}$ & $\hat{\sigma}_{\hat{\theta}}$ \\
\hline & & 2,909 & 0,154 & 2,966 & 0,121 \\
\hline & $\{2,0\}$ & 1,602 & 0,072 & 1,646 & 0,057 \\
\hline & $\{5,0\}$ & 3,459 & 0,190 & 3,587 & 0,149 \\
\hline$\phi_{1,1}$ & $\{1,0\}$ & 2,233 & 0,280 & 2,076 & 0,206 \\
\hline$\theta_{1,1}^{(\boldsymbol{\delta})}$ & $\{1,0\}$ & 2,057 & 0,101 & 1,930 & 0,072 \\
\hline$\theta_{2,2}^{(\boldsymbol{\delta})}$ & $\{1,0\}$ & 0,418 & 0,118 & 0,531 & 0,088 \\
\hline$\theta_{3,3}^{(\boldsymbol{\delta})}$ & $\{1,0\}$ & 1,345 & 0,094 & 1,318 & 0,072 \\
\hline$\theta_{4,4}^{(\boldsymbol{\delta})}$ & $\{1,0\}$ & 1,517 & 0,154 & 1,327 & 0,115 \\
\hline
\end{tabular}


Tabela 5.22: Estatísticas dos mil valores obtidos via simulação para os indicadores clássicos e robustos do MEE descrito pela Figura 5.2, por tamanho de amostra e com fração de $25 \%$ de contaminação proveniente da lei Uniforme Continua em ]-5;5/.

\begin{tabular}{|c|c|c|c|c|c|c|c|c|c|}
\hline \multirow{3}{*}{$\begin{array}{l}\text { Îndice } \\
\text { SRMR }\end{array}$} & \multirow{3}{*}{$\begin{array}{c}\text { Referência } \\
{[0 ; 0,08[}\end{array}$} & \multicolumn{4}{|c|}{$n=100$} & \multicolumn{4}{|c|}{$n=200$} \\
\hline & & $\hat{\mu}$ & $\hat{\sigma}$ & $\hat{\pi}$ & $\hat{\sigma}_{\hat{\pi}}$ & $\hat{\mu}$ & $\hat{\sigma}$ & $\hat{\pi}$ & $\hat{\sigma}_{\hat{\pi}}$ \\
\hline & & 0,035 & 0,009 & 0,000 & 0,000 & 0,049 & 0,007 & 0,001 & 0,032 \\
\hline RMSEA & {$[0 ; 0,08[$} & 0,227 & 0,049 & 0,995 & 0,071 & 0,391 & 0,040 & 1,000 & 0,000 \\
\hline NFI & ] $0,90 ; 1]$ & 0,973 & 0,010 & 0,012 & 0,109 & 0,939 & 0,012 & 0,820 & 0,384 \\
\hline IFI & ] $0,90 ; 1]$ & 0,977 & 0,010 & 0,006 & 0,077 & 0,941 & 0,012 & 0,780 & 0,414 \\
\hline NNFI & ] $0,90 ; 1]$ & 0,930 & 0,029 & 0,736 & 0,441 & 0,821 & 0,037 & 1,000 & 0,000 \\
\hline CFI & ] $0,90 ; 1]$ & 0,977 & 0,010 & 0,006 & 0,077 & 0,940 & 0,012 & 0,788 & 0,409 \\
\hline RNI & ]0,90;1] & 0,977 & 0,010 & 0,006 & 0,077 & 0,940 & 0,012 & 0,788 & 0,409 \\
\hline $\mathrm{GFI}_{\mathrm{MV}}$ & ] $0,90 ; 1]$ & 0,943 & 0,017 & 0,636 & 0,481 & 0,877 & 0,019 & 1,000 & 0,000 \\
\hline $\mathrm{GFI}_{\mathrm{MV}}^{\mathrm{GKO}}$ & ] $0,90 ; 1]$ & 0,860 & 0,177 & 0,707 & 0,455 & 0,926 & 0,066 & 0,528 & 0,499 \\
\hline $\mathrm{GFI}_{\mathrm{MV}}^{\mathrm{EVM}}$ & ] $0,90 ; 1]$ & 0,961 & 0,099 & 0,178 & 0,383 & 0,981 & 0,024 & 0,087 & 0,282 \\
\hline $\mathrm{GFI}_{\mathrm{MV}}^{\mathrm{CDM}}$ & ] $0,90 ; 1]$ & 0,969 & 0,058 & 0,141 & 0,348 & 0,965 & 0,032 & 0,273 & 0,446 \\
\hline $\mathrm{GFI}_{\mathrm{MV}}^{\mathrm{S}}$ & ] $0,90 ; 1]$ & 0,983 & 0,036 & 0,070 & 0,255 & 0,994 & 0,008 & 0,004 & 0,063 \\
\hline $\mathrm{GFI}_{\mathrm{MV}}^{\mathrm{MM}}$ & ] $0,90 ; 1]$ & 0,997 & 0,005 & 0,002 & 0,045 & 0,998 & 0,002 & 0,000 & 0,000 \\
\hline $\mathrm{AGFI}_{\mathrm{MV}}$ & ] $0,90 ; 1]$ & 0,716 & 0,087 & 1,000 & 0,000 & 0,385 & 0,093 & 1,000 & 0,000 \\
\hline $\mathrm{AGFI}_{\mathrm{MV}}^{\mathrm{GKO}}$ & ] $0,90 ; 1]$ & 0,302 & 0,886 & 0,950 & 0,218 & 0,631 & 0,330 & 0,939 & 0,239 \\
\hline $\mathrm{AGFI}_{\mathrm{MV}}^{\mathrm{EVM}}$ & ] $0,90 ; 1]$ & 0,804 & 0,497 & 0,783 & 0,412 & 0,903 & 0,118 & 0,523 & 0,500 \\
\hline $\mathrm{AGFI}_{\mathrm{MV}}^{\mathrm{CDM}}$ & ] $0,90 ; 1]$ & 0,843 & 0,292 & 0,781 & 0,414 & 0,826 & 0,158 & 0,746 & 0,436 \\
\hline $\mathrm{AGFI}_{\mathrm{MV}}^{\mathrm{S}}$ & ] $0,90 ; 1]$ & 0,913 & 0,180 & 0,436 & 0,496 & 0,971 & 0,038 & 0,154 & 0,361 \\
\hline \multirow[t]{2}{*}{$\mathrm{AGFI}_{\mathrm{MV}}^{\mathrm{MM}}$} & ] $0,90 ; 1]$ & 0,984 & 0,026 & 0,066 & 0,248 & 0,992 & 0,011 & 0,013 & 0,113 \\
\hline & & \multicolumn{4}{|c|}{$n=300$} & \multicolumn{4}{|c|}{$n=500$} \\
\hline Índice & Referência & $\hat{\mu}$ & $\hat{\sigma}$ & $\hat{\pi}$ & $\hat{\sigma}_{\hat{\pi}}$ & $\hat{\mu}$ & $\hat{\sigma}$ & $\hat{\pi}$ & $\hat{\sigma}_{\hat{\pi}}$ \\
\hline SRMR & {$[0 ; 0,08[$} & 0,048 & 0,006 & 0,000 & 0,000 & 0,049 & 0,004 & 0,000 & 0,000 \\
\hline RMSEA & {$[0 ; 0,08[$} & 0,395 & 0,032 & 1,000 & 0,000 & 0,398 & 0,025 & 1,000 & 0,000 \\
\hline NFI & ]0,90;1] & 0,939 & 0,010 & 0,883 & 0,322 & 0,938 & 0,008 & 0,946 & 0,226 \\
\hline IFI & ] $0,90 ; 1]$ & 0,940 & 0,010 & 0,850 & 0,357 & 0,939 & 0,008 & 0,934 & 0,248 \\
\hline NNFI & ] $0,90 ; 1]$ & 0,819 & 0,029 & 1,000 & 0,000 & 0,816 & 0,023 & 1,000 & 0,000 \\
\hline CFI & ] $0,90 ; 1]$ & 0,940 & 0,010 & 0,856 & 0,351 & 0,939 & 0,008 & 0,935 & 0,247 \\
\hline RNI & ] $0,90 ; 1]$ & 0,940 & 0,010 & 0,856 & 0,351 & 0,939 & 0,008 & 0,935 & 0,247 \\
\hline $\mathrm{GFI}_{\mathrm{MV}}$ & ]0,90;1] & 0,877 & 0,014 & 1,000 & 0,000 & 0,876 & 0,011 & 1,000 & 0,000 \\
\hline $\mathrm{GFI}_{\mathrm{MV}}^{\mathrm{GKO}}$ & ] $0,90 ; 1]$ & 0,916 & 0,061 & 0,658 & 0,475 & 0,935 & 0,038 & 0,572 & 0,495 \\
\hline $\mathrm{GFI}_{\mathrm{MV}}^{\mathrm{EVM}}$ & ] $0,90 ; 1]$ & 0,987 & 0,013 & 0,029 & 0,168 & 0,982 & 0,019 & 0,076 & 0,265 \\
\hline $\mathrm{GFI}_{\mathrm{MV}}^{\mathrm{CDM}}$ & ] $0,90 ; 1]$ & 0,989 & 0,012 & 0,018 & 0,133 & 0,944 & 0,032 & 0,595 & 0,491 \\
\hline $\mathrm{GFI}_{\mathrm{MV}}^{\mathrm{S}}$ & ] $0,90 ; 1]$ & 0,996 & 0,005 & 0,001 & 0,032 & 0,997 & 0,002 & 0,000 & 0,000 \\
\hline $\mathrm{GFI}_{\mathrm{MV}}^{\mathrm{MM}}$ & ] $0,90 ; 1]$ & 0,999 & 0,001 & 0,000 & 0,000 & 0,999 & 0,001 & 0,000 & 0,000 \\
\hline $\mathrm{AGFI}_{\mathrm{MV}}$ & ] $0,90 ; 1]$ & 0,384 & 0,072 & 1,000 & 0,000 & 0,378 & 0,057 & 1,000 & 0,000 \\
\hline $\mathrm{AGFI}_{\mathrm{MV}}^{\mathrm{GKO}}$ & ] $0,90 ; 1]$ & 0,579 & 0,305 & 0,980 & 0,140 & 0,676 & 0,192 & 0,991 & 0,094 \\
\hline $\mathrm{AGFI}_{\mathrm{MV}}^{\mathrm{EVM}}$ & ] $0,90 ; 1]$ & 0,937 & 0,067 & 0,430 & 0,495 & 0,910 & 0,097 & 0,512 & 0,500 \\
\hline $\mathrm{AGFI}_{\mathrm{MV}}^{\mathrm{CDM}}$ & ] $0,90 ; 1]$ & 0,946 & 0,060 & 0,374 & 0,484 & 0,718 & 0,159 & 0,877 & 0,329 \\
\hline $\mathrm{AGFI}_{\mathrm{MV}}^{\mathrm{S}}$ & ] $0,90 ; 1]$ & 0,980 & 0,026 & 0,077 & 0,267 & 0,986 & 0,012 & 0,021 & 0,143 \\
\hline $\mathrm{AGFI}_{\mathrm{MV}}^{\mathrm{MM}}$ & ] $0,90 ; 1]$ & 0,994 & 0,006 & 0,002 & 0,045 & 0,995 & 0,003 & 0,000 & 0,000 \\
\hline
\end{tabular}




\section{Capítulo 6}

\section{Considerações e Trabalhos Futuros}

Este trabalho teve o objetivo de examinar o impacto do uso de dados perturbados por outliers nas estimativas dos parâmetros dos Modelos de Equações Estruturais (MEE) e também de mensurar qual é a capacidade de detecção desse tipo de violação em uma vasta quantidade de indicadores de diagnóstico acerca do ajuste construídas a partir da função de discrepância ou da matriz de covariâncias amostrais, com destaque às propostas de medidas baseadas no Índice da Qualidade do Ajuste (ou GFI, de Goodness-of-Fit Index) e no Índice Corrigido da Qualidade do Ajuste (ou AGFI, de Adjusted Goodness-of-Fit Index) em suas expressões para o método de Máxima Verossimilhança, cujas adaptações consistiram em substituir a matriz de covariâncias clássicas pelas matrizes de covariâncias computadas com os estimadores robustos Elipsoide de Volume Mínimo, Covariância de Determinante Mínimo, S, MM e Gnanadesikan-Kettenring Ortogonalizado (GKO).

Pela avaliação conjunta dos resultados encontrados para desvios de simetria e excesso de curtose em diferentes frações de contaminação (5\% e 25\%), tamanhos de amostra (100, 200, 300 e 500) e quantidades de variáveis observadas perturbadas (4 e 8), as medidas GFI e AGFI modificadas pela matriz de covariâncias estimada pelo método GKO foram as únicas que conseguiram ser informativas em todas essas situações, devendo-se escolher a primeira ou a segunda quando a quantidade de parâmetros a serem estimados é baixa ou elevada, respectivamente.

Essas duas novas medidas são as principais contribuições à análise de outliers propostas nesta dissertação, pois, quando apuradas com cuidado, podem alertar que as estimativas dos parâmetros de um MEE estão comprometidas em decorrência de baixas proporções de contaminação existentes em poucas variáveis observadas, mesmo quando o tamanho amostral é grande. A saber, os demais indicadores erroneamente sugerem que o ajuste está muito bom nesse contexto.

No entanto, o uso desses novos dois índices não deve ser feito de maneira isolada, por conta de dois aspectos negativos: eles têm variabilidade alta e podem não deixar claro ao leitor sobre quão bem ajustado está o MEE quando seus valores pertencem à faixa que vai de 0,90 até 0,93. Assim, cabe ao pesquisador tomar os resultados das medidas aqui propostas e confrontá-los com os achados fornecidos por outras técnicas de diagnóstico, outros indicadores e por estudos adicionais sobre as propriedades associadas à distribuição empírica dos outliers, para então ter condições de dar um parecer fidedigno sobre a qualidade dos dados e a bondade do ajuste.

Havendo evidências bem embasadas de que as estimativas dos parâmetros do MEE estão afetadas por outliers, pode-se aplicar alguns métodos robustos de estimação para atenuar ou mesmo contornar o efeito das distorções que tais pontos provocam (Cirillo e Barroso, 2012b), uma vez que a exclusão de unidades amostrais sem erro não deve ser praticada. 
Finalmente, sugere-se para pesquisas futuras que:

- As versões das medidas GFI e AGFI adaptadas pela matriz GKO de covariâncias amostrais tenham seus comportamentos avaliados sob outros tipos de perturbações que podem ocorrer em dados reais, como multicolinearidade e não normalidade multivariada.

- Seja desenvolvida uma versão robusta para o índice Raiz Quadrada Média Padronizada (ou SRMR, de Standardized Root Mean Square Residual) que utilize as entradas da matriz GKO de covariâncias amostrais em substituição aos desvios-padrão e às covariâncias da matriz de covariâncias ordinárias ou clássicas que aparecem na expressão usual desse indicador. 


\section{Apêndice A}

\section{Derivadas de Matrizes}

A seguir, algumas propriedades sobre derivadas de matrizes, retiradas de Mulaik (2009a).

Seja $x$ um escalar. Sejam $\boldsymbol{A}$ e $\boldsymbol{B}$ duas matrizes com dimensões respectivamente iguais a $n \times r$ e $p \times q$. Também sejam $\boldsymbol{C}$ e $\boldsymbol{D}$ duas matrizes quadradas e não singulares, ambas com dimensões iguais a $n \times n$, com $\boldsymbol{C}$ constante.

Propriedade A.1. A derivada de $\boldsymbol{A}$ em relação a $x$ é uma matriz cujos elementos são as derivadas das entradas $a_{i, j}$ de $\boldsymbol{A}$ em relação a $x$ :

$$
\forall(i, j) \in\{1, \ldots, n\} \times\{1, \ldots, r\}, \quad \frac{\partial}{\partial x} \boldsymbol{A}=\left[\frac{\partial}{\partial x} a_{i, j}\right]_{n \times r} .
$$

Propriedade A.2. A derivada da soma de matrizes de mesmas dimensões em relação a $x$ é aditiva:

$$
(n=p) \wedge(r=q) \quad \Rightarrow \quad \frac{\partial}{\partial x}(\boldsymbol{A}+\boldsymbol{B})=\frac{\partial}{\partial x} \boldsymbol{A}+\frac{\partial}{\partial x} \boldsymbol{B} .
$$

Propriedade A.3. Vale a seguinte relaçấo para a derivada de produtos matriciais em relaçấo a $x$ :

$$
r=p \quad \Rightarrow \quad \frac{\partial}{\partial x} \boldsymbol{A} \boldsymbol{B}=\boldsymbol{A}\left(\frac{\partial}{\partial x} \boldsymbol{B}\right)+\left(\frac{\partial}{\partial x} \boldsymbol{A}\right) \boldsymbol{B}
$$

Propriedade A.4. Vale a seguinte relação para a derivada do traço do produto de uma matriz retangular por uma matriz quadrada, constante e não singular em relação a $x$ :

$$
\frac{\partial}{\partial x} \operatorname{tr}(\boldsymbol{A C})=\left[\operatorname{tr}\left(\frac{\partial}{\partial x} \boldsymbol{A}\right)\right] \boldsymbol{C} .
$$

Propriedade A.5. Vale a seguinte relação para a derivada de matrizes quadradas não singulares em relação a $x$ :

$$
\frac{\partial}{\partial x} \boldsymbol{D}^{-1}=-\boldsymbol{D}^{-1}\left(\frac{\partial}{\partial x} \boldsymbol{D}\right) \boldsymbol{D}^{-1}
$$

Propriedade A.6. Vale a seguinte relação para a derivada do traço do produto do inverso de uma matriz quadrada por uma matriz constante também quadrada em relação a $x$ :

$$
\frac{\partial}{\partial x} \operatorname{tr}\left(\boldsymbol{D}^{-1} \boldsymbol{C}\right)=-\operatorname{tr}\left(\boldsymbol{D}^{-1}\left(\frac{\partial}{\partial x} \boldsymbol{D}\right) \boldsymbol{D}^{-1} \boldsymbol{C}\right)=-\operatorname{tr}\left(\left(\frac{\partial}{\partial x} \boldsymbol{D}\right) \boldsymbol{D}^{-1} \boldsymbol{C} \boldsymbol{D}^{-1}\right) .
$$


Propriedade A.7. Vale a seguinte relação para a derivada do logaritmo natural do determinante de uma matriz quadrada não singular em relação a $x$ :

$$
\frac{\partial}{\partial x} \ln \operatorname{det} \boldsymbol{D}=-\left[\operatorname{tr}\left(\boldsymbol{D}^{-1}\right)\right]\left(\frac{\partial}{\partial x} \boldsymbol{D}^{\prime}\right) .
$$




\section{Apêndice B}

\section{Algoritmos de Estimadores Robustos}

A seguir, algumas propostas de algoritmo para o cálculo dos estimadores robustos M, MMQ, MQP e GKO serão revisadas.

Os leitores interessados no tópico de algoritmos para a computação dos estimadores robustos EVM, CDM, S e MM de regressão/locação e escala são encorajados a ler Maronna et al. (2006) e Yohai (1987). Essas matrizes de covariâncias robustas podem ser calculadas a partir das funções CovMve, CovMcd, CovSest e CovMMest, todas pertencentes à biblioteca rrcov do ambiente computacional R 2.15.2 (Todorov e Filzmoser, 2009).

\section{B.1 M-Estimador}

Uma forma de computar as M-Estimativas de regressão e escala é a partir do algoritmo de Dutter (1977), tal como descrito em Souza (1998). Ele consiste na execução dos seguintes passos:

1. Calcule estimativas iniciais $\hat{\beta}$ e $\hat{\sigma}$ sequencialmente para $\beta$ e $\sigma$ usando o método de Mínimos Quadrados Ordinários ou, preferencialmente, o estimador robusto da Norma $\mathrm{L}_{1}$.

2. Sejam $\hat{\beta}^{(0)}=\hat{\beta}=\left[\begin{array}{lll}\hat{\beta}_{1}^{(0)} & \ldots & \hat{\beta}_{q}^{(0)}\end{array}\right]^{\prime}$ e $\hat{\sigma}^{(0)}=\hat{\sigma} / 0,6745$ as respectivas estimativas iniciais para $\beta$ e $\sigma$. Atualize o estimador de escala pela fórmula

$$
\left[\hat{\sigma}^{(1)}\right]^{2}=\frac{1}{0,7785(n-q-1)} \sum_{k=1}^{n} \psi^{2}\left(\frac{y_{k}-\hat{\beta}_{1}^{(0)} x_{1}-\cdots-\hat{\beta}_{q}^{(0)} x_{q}}{\hat{\sigma}^{(0)}}\right)\left[\hat{\sigma}^{(0)}\right]^{2},
$$

em que $\psi(u)=\rho^{\prime}(u)$ é a derivada da função de Huber de parâmetro $c=1,5$.

3. Calcule os resíduos winsorizados ${ }^{1} \Delta_{k}^{(0)}$ :

$$
\forall k \in\{1, \ldots, n\}, \quad \Delta_{k}^{(0)}=\psi\left(\frac{y_{k}-\hat{\beta}_{1}^{(0)} x_{1}-\cdots-\hat{\beta}_{q}^{(0)} x_{q}}{\hat{\sigma}^{(1)}}\right) \hat{\sigma}^{(1)} .
$$

4. Seja $\Delta^{(0)}=\left[\begin{array}{lllll}\Delta_{1}^{(0)} & \cdots & \Delta_{k}^{(0)} & \cdots & \Delta_{n}^{(0)}\end{array}\right]^{\prime}$ o vetor de resíduos winsorizados e recorde que

\footnotetext{
${ }^{1}$ Esses resíduos têm o objetivo de amortecer o impacto do resíduo $y_{k}-\hat{\beta}_{1}^{(0)} x_{1}-\cdots-\hat{\beta}_{q}^{(0)} x_{q}$, se este for muito grande. Para mais detalhes sobre este tópico, consulte Bustos (1981).
} 
$X$ é a mesma matriz descrita pela expressão (3.8). Calcule $\hat{\beta}^{(1)}=\hat{\beta}^{(0)}+1,1542 E^{(0)}$, em que:

$$
E^{(0)}=\left(X^{\prime} X\right)^{-1} X^{\prime} \Delta^{(0)}
$$

5. Repita os Passos $1-4$ com $\hat{\beta}^{(1)}$ e $\hat{\sigma}^{(1)}$ no lugar de $\hat{\beta}^{(0)}$ e $\hat{\sigma}^{(0)}$ para obter $\hat{\beta}^{(2)}$ e $\hat{\sigma}^{(2)}$, respectivamente. Interrompa o processo na $\kappa$-ésima $(\kappa \in \mathbb{N})$ iteração que atender simultaneamente a duas seguintes condições:

$$
\forall j \in\{1, \ldots, q\}, \quad\left\{\begin{array}{cc}
1,1542 \cdot\left|E_{j}^{(\kappa)}\right| & <\epsilon \hat{\sigma}^{(\kappa+1)} \sqrt{a_{j, j}} \\
\left|\hat{\sigma}^{(\kappa+1)}-\hat{\sigma}^{(\kappa)}\right|<c & <\hat{\sigma}^{(\kappa+1)}
\end{array}\right.
$$

em que $\epsilon$ e $\iota$ são níveis de tolerância positivos, $E_{j}^{(\kappa)}$ é a $j$-ésima componente de $E^{(\kappa)}$ e $a_{j, j}$ é o $j$-ésimo elemento da diagonal de $\left(X^{\prime} X\right)^{-1}$. As M-Estimativas de $\beta$ e $\sigma$ são dadas por $\hat{\beta}^{(\kappa+1)}$ e $\hat{\sigma}^{(\kappa+1)}$, respectivamente.

No R, pode-se recorrer à função $r l m$, da biblioteca $M A S S$, para computar as M-Estimativas de regressão e escala (Venables e Ripley, 2002).

\section{B.2 Menor Mediana dos Quadrados e Mediana dos Quadrados Po- dados}

A computação das estimativas de Menor Mediana dos Quadrados (MMQ) é dada pela execução dos passos a seguir:

1. Determine todos os $\left(\begin{array}{l}n \\ q\end{array}\right)$ subconjuntos de tamanho $q$ de $\{1, \ldots, n\}$.

2. Para cada um dos $\left(\begin{array}{l}n \\ q\end{array}\right)$ subconjuntos $\left\{i_{1}, \ldots, i_{q}\right\}$, compute estimativas $\hat{\beta}$ de Mínimos Quadrados Ordinários ou, preferencialmente, de $\mathrm{L}_{1}$.

3. Calcule os resíduos associados a cada uma das estimativas obtidas no passo anterior e observe o valor do resíduo mediano correspondente a cada subconjunto.

4. Identifique qual subconjunto $\left\{i_{1}, \ldots, i_{q}\right\}$ gerou o menor valor de resíduo mediano. A estimativa $\hat{\beta}$ de MMQ é a fornecida por este subconjunto.

De modo bastante similar, as estimativas de Mínimos Quadrados Podados (MQP) podem ser obtidas com a realização das seguintes etapas:

1. Determine todos os $\left(\begin{array}{l}n \\ q\end{array}\right)$ subconjuntos de tamanho $q$ de $\{1, \ldots, n\}$.

2. Para cada um dos $\left(\begin{array}{l}n \\ q\end{array}\right)$ subconjuntos $\left\{i_{1}, \ldots, i_{q}\right\}$, compute estimativas $\hat{\beta}$ de Mínimos Quadrados Ordinários ou, preferencialmente, de $\mathrm{L}_{1}$.

3. Calcule os resíduos associados a cada uma das estimativas obtidas no passo anterior, ordene as componentes de cada vetor de resíduos e observe o valor da soma do quadrado dos $h$ primeiros resíduos correspondente a cada subconjunto. 
4. Identifique qual subconjunto $\left\{i_{1}, \ldots, i_{q}\right\}$ gerou o menor valor daquela soma de resíduos. A estimativa $\hat{\beta}$ de MQP é a fornecida por este subconjunto.

No $\mathrm{R}$, pode-se recorrer à função $l q s$, da biblioteca $M A S S$, para computar estimativas de regressão e escala dos estimadores MMQ e MQP, inserindo os respectivos argumentos method="lms" e method="lts" (Venables e Ripley, 2002).

Alternativamente, subconjuntos de tamanho $q+1$ dos $n$ pontos poderiam ser considerados na programação dos algoritmos MMQ e MQP, para que haja pelo menos um grau de liberdade ao fazer inferências sobre os $q$ parâmetros ( $q$ variáveis, ou $q-1$ variáveis e um intercepto) do modelo linear de regressão usando o subconjunto ótimo de tamanho $q+1$. (Bulhões e Lima, 2010).

\section{B.3 Gnanadesikan-Kettenring Ortogonalizado}

Nesta seção, serão revisados os passos apresentados em Rocha (2010) para calcular as estimativas de locação e escala pelo método de Gnanadesikan-Kettenring Ortogonalizado (GKO).

Para uma matriz $\boldsymbol{Z}=\left[z_{k, j}\right]_{n \times(p+q)}$ das $n$ observações em $p+q$ variáveis observadas, denote cada uma das $n$ linhas por $\boldsymbol{z}_{k}^{\prime}(k \in\{1, \ldots, n\})$ e represente por $\boldsymbol{z}_{j}(j \in\{1, \ldots, p+q\})$ cada uma das $p+q$ colunas. As estimativas GKO de locação $\hat{\boldsymbol{\mu}}(\boldsymbol{Z})$ e escala $\hat{\boldsymbol{\Sigma}}(\boldsymbol{Z})$ podem ser obtidas a partir da execução das etapas a seguir:

1. Calcule uma matriz $\boldsymbol{B}$ de dados normalizados, formada por colunas $\boldsymbol{b}_{j}=\boldsymbol{z}_{j} / \hat{\sigma}\left(\boldsymbol{z}_{j}\right)$ e também por linhas $\boldsymbol{b}_{k}^{\prime}=\left[\operatorname{diag}\left\{1 / \hat{\sigma}\left(\boldsymbol{z}_{1}\right), \ldots, 1 / \hat{\sigma}\left(\boldsymbol{z}_{p+q}\right)\right\} \cdot\left(\boldsymbol{z}_{k}^{\prime}\right)^{\prime}\right]^{\prime}$, em que $\hat{\sigma}(\cdot)$ é uma medida de dispersão robusta univariada, como o desvio-padrão aparado, por exemplo.

2. Obtenha a matriz $\boldsymbol{U}=\left[U_{i, j}\right]_{(p+q) \times(p+q)}$, com:

$$
\forall i, j \in\{1, \ldots, p+q\}, \quad U_{i, j}=\left\{\begin{array}{cc}
1, & i=j \\
\frac{\hat{\sigma}^{2}\left(\boldsymbol{b}_{i}+\boldsymbol{b}_{j}\right)-\hat{\sigma}^{2}\left(\boldsymbol{b}_{i}-\boldsymbol{b}_{j}\right)}{4}, & i \neq j
\end{array} .\right.
$$

3. Compute os $p+q$ valores próprios $\lambda_{j}$ e os correspondentes $p+q$ vetores próprios $\boldsymbol{e}_{j}$ de $\boldsymbol{U}$, para então obter as matrizes $\boldsymbol{E}=\left[\begin{array}{lll}\boldsymbol{e}_{1} & \cdots & \boldsymbol{e}_{p+q}\end{array}\right]$ e $\Lambda=\operatorname{diag}\left\{\lambda_{1}, \ldots, \lambda_{p+q}\right\}$.

4. Calcule a matriz $\boldsymbol{V}$, formada pelas $n$ linhas $\boldsymbol{v}_{k}^{\prime}$, tais que:

$$
\forall k \in\{1, \ldots, n\}, \quad \boldsymbol{v}_{k}^{\prime}=\left[\boldsymbol{E}^{\prime}\left(\boldsymbol{b}_{k}^{\prime}\right)^{\prime}\right]^{\prime}=\left[\boldsymbol{E}^{\prime} \cdot \operatorname{diag}\left\{1 / \hat{\sigma}\left(\boldsymbol{z}_{1}\right), \ldots, 1 / \hat{\sigma}\left(\boldsymbol{z}_{p+q}\right)\right\} \cdot\left(\boldsymbol{z}_{k}^{\prime}\right)^{\prime}\right]^{\prime} .
$$

5. Para todo $j \in\{1, \ldots, p+q\}$, calcule $\hat{\mu}\left(\boldsymbol{v}_{j}\right)$ e $\hat{\sigma}\left(\boldsymbol{v}_{j}\right)$, em que $\hat{\mu}(\cdot)$ é uma medida de locação robusta univariada, como a mediana, por exemplo. Em seguida, compute as seguintes matrizes:

$$
\boldsymbol{\Gamma}=\operatorname{diag}\left\{\hat{\sigma}^{2}\left(\boldsymbol{v}_{1}\right), \ldots, \hat{\sigma}^{2}\left(\boldsymbol{v}_{p+q}\right)\right\}
$$

e

$$
\mathbf{\Upsilon}=\left[\begin{array}{lll}
\hat{\mu}\left(\boldsymbol{v}_{1}\right) & \cdots & \hat{\mu}\left(\boldsymbol{v}_{p+q}\right)
\end{array}\right]^{\prime} .
$$


6. Seja $\boldsymbol{A}=\operatorname{diag}\left\{\hat{\sigma}\left(\boldsymbol{z}_{1}\right), \ldots, \hat{\sigma}\left(\boldsymbol{z}_{p+q}\right)\right\} \cdot \boldsymbol{E}$. Calcule $\boldsymbol{Z}$ a partir das $n$ linhas $\boldsymbol{z}_{k}^{\prime}=\left[\boldsymbol{A}\left(\boldsymbol{v}_{k}^{\prime}\right)^{\prime}\right]^{\prime}$ e, finalmente, obtenha as estimativas GKO desejadas:

$$
\hat{\Sigma}(Z)=A \Gamma A^{\prime}
$$

$\mathrm{e}$

$$
\hat{\mu}(Z)=A \Upsilon .
$$

Usando o R, a matriz de covariâncias GKO pode ser computada por meio da função CovOgk, da biblioteca rrcov (Todorov e Filzmoser, 2009). 


\section{Apêndice C}

\section{Programas em R das Simulações}

\section{C.1 Cenário 1}

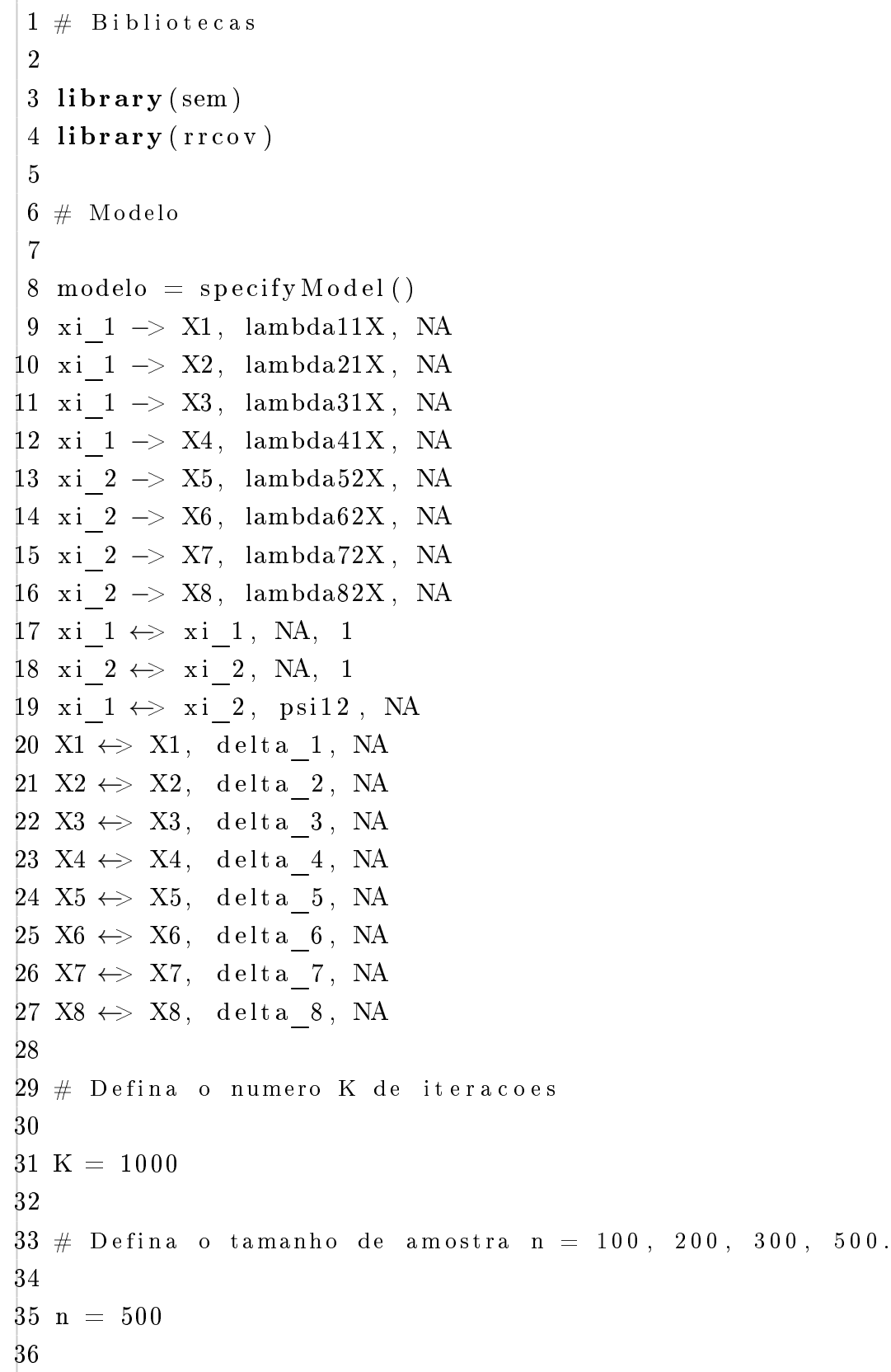


38 \# Armazenando os resultados

39

40 Coeficientes $=\operatorname{matrix}($ data $=\operatorname{rep}(0,17 * \mathrm{~K})$, nrow $=\mathrm{K}, \quad$ ncol $=17$,

41 dimnames $=1$ ist $(1: \mathrm{K}, \mathbf{c}$ ("lamb11", "lamb21", "lamb31", "lamb41",

42 "lamb52", "lamb62", "lamb72", "lamb82",

43 "phi_12", "thded11", "thde22", "thde33", "thde44",

44 "thde55", "thde66", "thde77", "thde88")))

45

46 Indices $=\operatorname{matrix}($ data $=\operatorname{rep}(0,19 * \mathrm{~K})$, nrow $=\mathrm{K}, \quad \mathbf{n c o l}=19$,

47 dimnames $=1$ is $\mathbf{t}(1: \mathrm{K}, \mathbf{c}($ "SRMR", "RMSEA", "NFI", "IFI",

48

"NNFI", "CFI", "RNI", "GFI_ORD", "GFI_GKO", "GFI_EVM",

"GFI_CDM", "GFI_S", "GFI_MM", "AGFI_ORD", "AGFI_GKO",

"AGFI_EVM", "AGFI_CDM", "AGFI_S", "AGFI_MM") ))

\# Iterando

53

4 for $(\mathrm{i}$ in $1: \mathrm{K})\{$

\# Para imprimir a i-esima iteracao

print (i)

flush.console ()

\# Usando o comando repetir para selecionar iteracoes convergentes e factiveis

repeat \{

\# A simulacao das variaveis que compoem o modelo

$\mathrm{xi} 1=\operatorname{rnorm}(\mathrm{n}, \quad 0,1)$

$\mathrm{xi} 2=0.2 * \mathrm{xi} 1+\operatorname{rnorm}(\mathrm{n}, 0,1)$

delta $1=\operatorname{rnorm}(\mathrm{n}, \quad 0,1)$

delta $2=\operatorname{rnorm}(\mathrm{n}, 0,1)$

delta $3=\operatorname{rnorm}(\mathrm{n}, 0,1)$

delta $4=\operatorname{rnorm}(\mathrm{n}, 0,1)$

delta $5=\operatorname{rnorm}(\mathrm{n}, 0,1)$

delta $6=\operatorname{rnorm}(\mathrm{n}, 0,1)$

delta $7=\operatorname{rnorm}(\mathrm{n}, 0,1)$

delta $8=\operatorname{rnorm}(n, 0,1)$

$\mathrm{X} 1=0.3 * \mathrm{xi} 1+$ delta 1

$\mathrm{X} 2=0.6 * \mathrm{xi} 1+$ del ta 2

$\mathrm{X} 3=0.9 * \mathrm{xi} 1+$ delta 3

$\mathrm{X} 4=1.2 * \mathrm{xi} 1+\operatorname{delta} 4$

$\mathrm{X} 5=0.7 *$ xi $2+$ delta 5

$\mathrm{X} 6=-1.8 * \mathrm{xi} 2+$ delta 6

$\mathrm{X} 7=-0.5 * \mathrm{xi} 2+$ delta 7

$\mathrm{X} 8=0.2 * \mathrm{xi} 2+\operatorname{delta} 8$

89 
90 \# Resumindo os dados:

91 
142 Indices $[\mathrm{i}, 16]=1-((8 *(8+1)) /(2 * \operatorname{summary}($ ajuste $) \$ \mathbf{d f})) *(1-\operatorname{Indices}[\mathrm{i}, 10])$

143 Indices $[\mathrm{i}, 17]=1-((8 *(8+1)) /(2 * \operatorname{summary}($ ajuste $) \$ \mathbf{d f})) *(1-\operatorname{Indices}[\mathrm{i}, 11])$

144 Indices $[\mathrm{i}, 18]=1-((8 *(8+1)) /(2 * \operatorname{summary}($ ajuste $) \$ \mathbf{d f})) *(1-\operatorname{Indices}[\mathrm{i}, 12])$

145 Indices $[\mathrm{i}, 19]=1-((8 *(8+1)) /(2 * \operatorname{summary}($ ajuste $) \$ \mathbf{d f})) *(1-\operatorname{Indices}[\mathrm{i}, 13])$

146

$147\}$ 


\section{C.2 Cenário 2}

1 \# Bibliotecas

2

3 library (sem)

4 library (rrcov)

5

6 \# Modelo

7

8 modelo $=$ matrix $(\mathbf{c}($

9 'xi1 $\rightarrow \mathrm{X} 1$ ', NA, 1 ,

10 'xi1 $\rightarrow \mathrm{X} 2$ ', ' $\operatorname{lamX12}$ ', NA,

11 'xi1 $\rightarrow \mathrm{X} 3{ }^{\prime}$, , ' $\operatorname{lamX13}$ ', NA,

$12^{\prime}$ xi1 $\rightarrow \mathrm{X} 4$ ', ' $\operatorname{lamX14}$ ', NA,

13 'xi1 $\leftrightarrow$ Eta1', 'phi11', NA,

14 'X1 $\leftrightarrow \mathrm{X} 1$ ', 'theX1', NA,

$15{ }^{\prime} \mathrm{X} 2 \leftrightarrow \mathrm{X} 2$ ', 'theX2', NA,

16 'X3 $\leftrightarrow \mathrm{X} 3{ }^{\prime}$, ' 'theX3', NA,

17 'X4 $\leftrightarrow \mathrm{X} 4$ ', 'theX4', NA),

$18 \operatorname{ncol}=3$, byrow $=\mathrm{T}$ )

19

20 \# Defina o numero $K$ de iteracoes

21

$22 \mathrm{~K}=1000$

23

24 \# Defina o tamanho de amostra $\mathrm{n}=100,200,300,500$.

25

$26 \mathrm{n}=100$

27

28

29 \# Armazenando os resultados

30

31 Coeficientes $=\operatorname{matrix}($ data $=\operatorname{rep}(0,8 * \mathrm{~K}), \quad$ nrow $=\mathrm{K}, \quad \mathbf{n c o l}=8$,

32 dimnames $=\mathbf{l i s t}(1: \mathrm{K}, \mathbf{c}($ "lamb2X", "lamb3X", "lamb4X", "phi11",

"thde1X", "thde $2 X "$, "thde $3 X "$, "thde $4 X "))$ )

34

35 Indices $=\operatorname{matrix}($ data $=\operatorname{rep}(0,19 * \mathrm{~K}), \quad$ nrow $=\mathrm{K}, \mathbf{n c o l}=19$,

36 dimnames $=\mathbf{l}$ ist $(1: \mathrm{K}, \mathbf{c}($ "SRMR", "RMSEA", "NFI", "IFI",

"NNFI", "CFI", "RNI", "GFI_ORD", "GFI_GKO", "GFI_EVM",

"GFI_CDM", "GFI_S", "GFI_MM", "AGFI_ORD" , "AGFI_GKO" ,

"AGFI_EVM", "AGFI_CDM", "AGFI_S", "AGFI_MM" )))

39

40

41 \# Variaveis contaminadas

42

43 alpha $=0.05$

44

45 Contaminacao1X $=\mathbf{c}(1.500391,3.674611,4.013004,0.811637,1.470367)$

46 Contaminacao2X $=\mathbf{c}(3.7420058,1.1095891,1.0918364,2.0881885,0.7768462)$

47 Contaminacao3X $=\mathbf{c}(1.2226308,3.9156339,0.6942794,0.1209132,0.2326593)$

48 Contaminacao4X $=\mathbf{c}(7.8857738,0.5595947,0.3272180,1.5052264,0.7226672)$

49

50 \# Iterando

51 


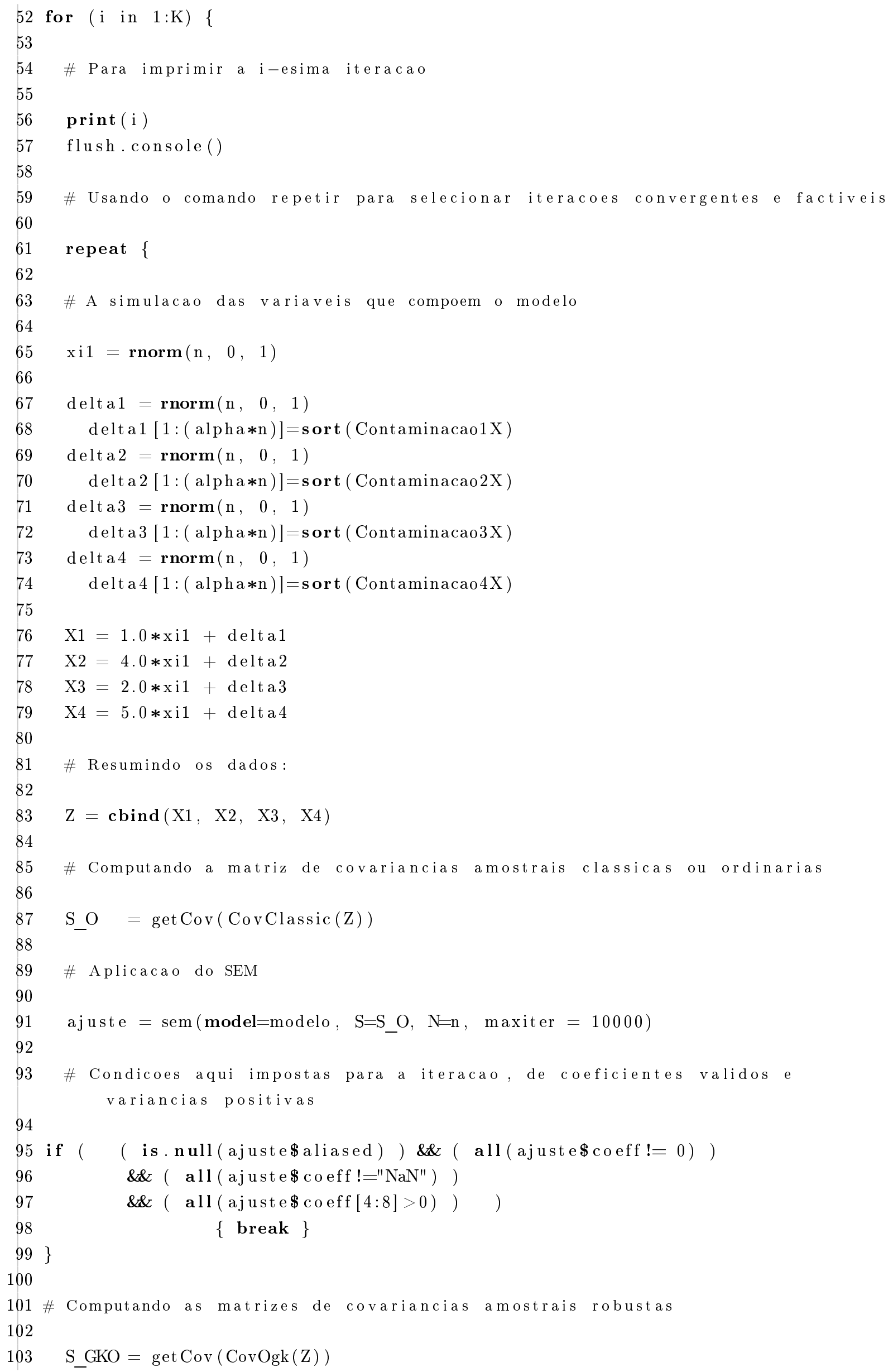


104

105

106

107

108

110

111

112

113

114

115

116

117

118

119

120

121

122

123

124

125

126

127

128

129

130

131

132

133

134

135

136

137

$138\}$

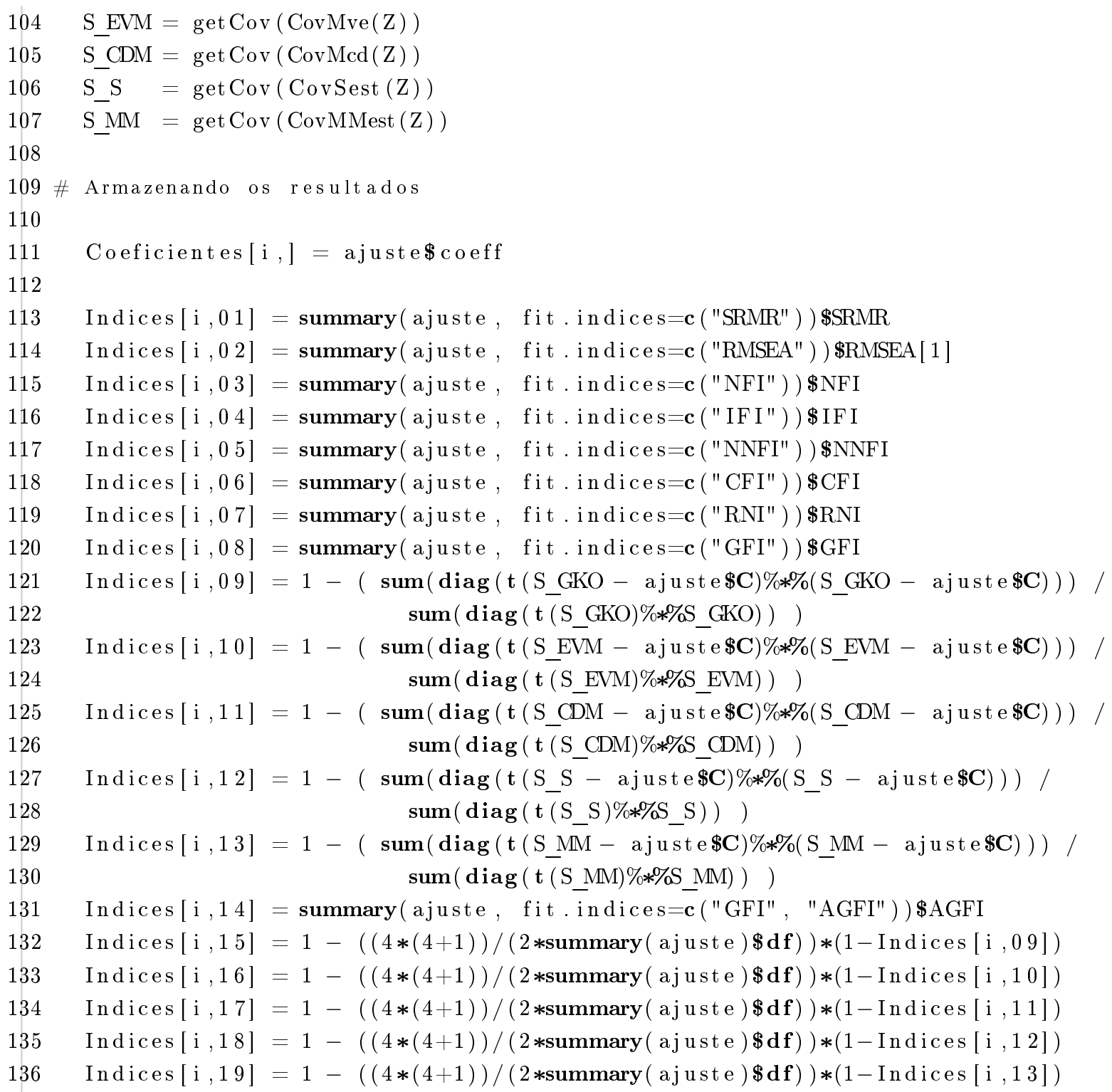


APÊNDICE C 


\section{Referências Bibliográficas}

Alexandrino-Silva et al. (2013) C. Alexandrino-Silva, Y. P. Wang, M. Carmen Viana, R. S. Bulhões, S. S. Martins e L. H. Andrade. Gender Differences in Symptomatic Profiles of Depression: Results from the São Paulo Megacity Mental Health Survey. Journal of Affective Disorders, 147 (1-3):355-364. ISSN 0165-0327. Citado na(s) página(s) 33

Amorim et al. (2010) L. D. A. F. Amorim, R. L. Fiaccone, C. A. S. T. Santos, T. N. Santos, L. T. L. P. Moraes, N. F. Oliveira, S. O. Barbosa, D. N. Santos, L. M. Santos, S. M. A. Matos e M. L. Barreto. Structural Equation Modeling in Epidemiology. Cadernos de Saúde Pública, 26 (12):2251-2262. Citado na(s) página(s) 8

Barnett e Lewis (1984) V. Barnett e T. Lewis. Outliers in Statistical Data. Wiley, Chichester. Citado na(s) página(s) 14

Bertossi (2012) E. Bertossi. semGOF: Goodness-of-Fit Indexes for Structural Equation Models, 2012. URL http://CRAN.R-project.org/package=semGOF. R package version 0.2-0. Citado na(s) página(s) 27

Bistaffa (2011) B. C. Bistaffa. Incorporação de Indicadores Categóricos Ordinais em Modelos de Equações Estruturais. Dissertação de Mestrado, Instituto de Matemática e Estatística, Universidade de São Paulo, São Paulo. Citado na(s) página(s) 12, 13, 32, 33

Blunch (2008) N. Blunch. Introduction to Structural Equation Modelling using SPSS and AMOS. Sage. Citado na(s) página(s) 11

Bollen (1989) K. A. Bollen. Structural Equations with Latent Variables. Wiley-Interscience, New York. Citado na(s) página(s) 3, 8, 10, 13, 23, 37

Bollen e Long (1993) K. A. Bollen e J. S. Long. Testing Structural Equation Models. Sage, Newbury Park. Citado na(s) página(s) 23

Bouroche e Saporta (1980) J. M. Bouroche e G. Saporta. L'analyse des Données. Presses Universitaires de France, Paris. Citado na(s) página(s) 16

Brunelli (2012) R. T. Brunelli. Análise do Impacto de Perturbações sobre Medidas de Qualidade de Ajuste para Modelos de Equações Estruturais. Dissertação de Mestrado, Instituto de Matemática e Estatística, Universidade de São Paulo, São Paulo. Citado na(s) página(s) 29, 30, 32

Bulhões e Lima (2010) R. S. Bulhões e V. M. C. Lima. Comparação de Estimadores de Regressão. Em $19^{o}$ Simpósio Nacional de Probabilidade e Estatística, São Pedro. Associação Brasileira de Estatística. Citado na(s) página(s) 16,69

Bustos (1981) O. H. Bustos. Estimação Robusta no Modelo de Posição. Instituto de Matemática Pura e Aplicada, Rio de Janeiro. Citado na(s) página(s) 67

Cirillo e Barroso (2012a) M. A. Cirillo e L. P. Barroso. Avaliação do Efeito de Outliers no Índice de Qualidade de Ajuste GFI em Modelos de Equações Estruturais e Propostas de Índices Alternativos. Em 20ㅡ Simpósio Nacional de Probabilidade e Estatística, João Pessoa. Associação Brasileira de Estatística. Citado na(s) página(s) 23, 29, 33, 36, 38, 51 
Cirillo e Barroso (2012b) M. A. Cirillo e L. P. Barroso. Robust Regression Estimates in the Prediction of Latent Variables in Structural Equation Models. Journal of Modern Applied Statistical Methods, 11(1):42-53. Citado na(s) página(s) 21, 63

Conceição (2004) E. L. T. Conceição. Uma Comparação por Simulação de Monte Carlo de Estimadores de Regressão Não-Linear Robustos em Problemas de Engenharia Química. Tese de Doutorado, Departamento de Engenharia Química, Universidade de Coimbra, Coimbra. Citado na(s) página(s) 14,18

Diamantopoulos e Siguaw (2000) A. Diamantopoulos e J. A. Siguaw. Introducing LISREL: A Guide for the Uninitiated. Sage, London. Citado na(s) página(s) 52

Dutter (1977) R. Dutter. Numerical Solution of Robust Regression Problems: Computational Aspects, a Comparison. Journal of Statistical Computation and Simulation, 5(3):207-238. Citado na(s) página(s) 67

Everitt (1984) B. S. Everitt. An Introduction to Latent Variable Models. Chapman and Hall, London. Citado na(s) página(s) 11

Fan e Sivo (2005) X. Fan e S. A. Sivo. Sensitivity of Fit Indexes to Misspecified Structural or Measurement Model Components: Rationale of Two-Index Strategy Revisited. Structural Equation Modeling, 12(3):343-367. Citado na(s) página(s) 24

Filzmoser (2002) P. Filzmoser. Robust Factor Analysis. Em G. A. Marcoulides e I. Moustaki, editors, Latent Variable and Latent Structure Models. Lawrence Erlbaum. Citado na(s) página(s) 19, 20

Fox (2006) J. Fox. Structural Equation Modeling with the sem Package in R. Structural Equation Modeling, 13(3):465-486. Citado na(s) página(s) 33

Fox et al. (2013) J. Fox, Z. Nie e J. Byrnes. sem: Structural Equation Models, 2013. URL http://CRAN.R-project.org/package=sem. R package version 3.1-0. Citado na(s) página(s) 33

Gonçalves e Mendes-Lopes (2000) E. Gonçalves e N. Mendes-Lopes. Probabilidades: Princípios Teóricos. Escolar Editora, Coimbra. Citado na(s) página(s) 14

Hair et al. (2010) J. F. Hair, R. E. Anderson, R. L. Tatham e W. C. Black. Multivariate Data Analysis. Prentice Hall, New Jersey. Citado na(s) página(s) 8, 32

Hallquist e Wiley (2013) M. Hallquist e J. Wiley. MplusAutomation: Automating Mplus Model Estimation and Interpretation, 2013. URL http://CRAN.R-project.org/package= MplusAutomation. R package version 0.5-4. Citado na(s) página(s) 33

Hatcher (1996) L. Hatcher. Using SAS ${ }^{\circledR}$ PROC CALIS for Path Analysis: An Introduction. Structural Equation Modeling: A Multidisciplinary Journal, 3(2):176-192. Citado na(s) página(s) 11

Hill e Hill (2005) M. Hill e A. Hill. Investigação por Questionário. Edições Sílabo, Lisboa. Citado na(s) página(s) 12

Hoaglin et al. (1992) D. C. Hoaglin, F. Mosteller e J. W. Tukey. Análise Exploratória de Dados. Técnicas Robustas - Um Guia. Edições Salamandra, Lisboa. Citado na(s) página(s) 14, 17

Hooper et al. (2008) D. Hooper, J. Coughlan e M. R. Mullen. Structural Equation Modelling: Guidelines for Determining Model Fit. Electronic Journal of Business Research Methods, 6(1): 53-60. Citado na(s) página(s) 32

Hox e Bechger (1998) J. J. Hox e T. M. Bechger. An Introduction to Structural Equation Modeling. Family Science Review, 11:354-373. Citado na(s) página(s) 31 
Hoyle e Panter (1995) R. H. Hoyle e A. T. Panter. Writing About Structural Equation Models. Em R. H. Hoyle, editor, Structural Equation Modeling: Concepts, Issues and Applications, páginas 159-176. Sage, California. Citado na(s) página(s) 31

Hubert e Vandervieren (2008) M. Hubert e E. Vandervieren. An Adjusted Boxplot for Skewed Distributions. Computational Statistics \& Data Analysis, 52(12):5186-5201. Citado na(s) página(s) 28

Kirby e Bollen (2009) J. B. Kirby e K. A. Bollen. Using Instrumental Variable Tests to Evaluate Model Specification in Latent Variable Structural Equation Models. Sociological Methodology, 39 (1):327-355. Citado na(s) página(s) 29

Latif (2000) S. A. Latif. Modelagem de Equações Estruturais. Dissertação de Mestrado, Instituto de Matemática e Estatística, Universidade de São Paulo, São Paulo. Citado na(s) página(s) 5, 13, 24

Lattin et al. (2011) J. Lattin, J. D. Carrol e P. E. Green. Análise de Dados Multivariados. Cegrage Learning, São Paulo. Citado na(s) página(s) 9, 19, 31

Leite e Singer (1990) J. G. Leite e J. M. Singer. Métodos Assintóticos em Estatística: Fundamentos e Aplicações. Em 9o Simpósio Nacional de Probabilidade e Estatística, São Paulo. Associação Brasileira de Estatística. Citado na(s) página(s) 9

Lima (2005a) A. L. M. C. Lima. Modelagem de Equações Estruturais: Um Método para a Análise de Fenômenos Complexos. Caderno CRH, 18(45):471-484. Citado na(s) página(s) 3

Lima (2005b) A. L. M. C. Lima. Modelagem de Equações Estruturais: Uma Contribuição Metodológica para o Estudo da Pobreza. Tese de Doutorado, Departamento de Ciências Sociais, Universidade Federal da Bahia, Salvador. Citado na(s) página(s) 8

Marôco (2010) J. Marôco. Análise de Equaçôes Estruturais: Fundamentos Teóricos, Software e Aplicações. ReportNumber, Pêro Pinheiro. Citado na(s) página(s) 6, 8, 11, 15, 25, 27, 33

Maronna et al. (2006) R. A. Maronna, R. D. Martin e V. J. Yohai. Robust Statistics - Theory and Methods. Wiley. Citado na(s) página(s) 18, 67

Marsh et al. (2004) H. W. Marsh, K. T. Hau e Z. Wen. In Search of Golden Rules: Comment on Hypothesis-Testing Approaches to Setting Cutoff Values for Fit Indexes and Dangers in Overgeneralizing Hu and Bentler's (1999) Findings. Structural Equation Modeling, 11(3):320341. Citado na(s) página(s) 36

McDonald e Hartmann (1992) R. P. McDonald e W. M. Hartmann. A Procedure for Obtaining Initial Values of Parameters in the RAM Model. Multivariate Behavioral Research, 27(1):57-76. Citado na(s) página(s) 33

Melhado (2004) T. T. Melhado. Medidas de Ajuste de Modelos de Equaçôes Estruturais. Dissertação de Mestrado, Instituto de Matemática e Estatística, Universidade de São Paulo, São Paulo. Citado na(s) página(s) 24, 25, 27

Melhado (2009) T. T. Melhado. Influência Local para Modelos de Equaçôes Estruturais com Distribuição Elíptica. Tese de Doutorado, Instituto de Matemática e Estatística, Universidade de São Paulo, São Paulo. Citado na(s) página(s) 8, 12

Mendes (1999) B. V. M. Mendes. Regressão Robusta: Conceitos, Aplicações e Aspectos Computacionais. Associação Brasileira de Estatística, São Paulo. Citado na(s) página(s) 14, 17, 18, 21, 28

Miranda (2009) H. A. S. R. Miranda. Métodos Robustos em Geoestatística. Tese de Doutorado, Departamento de Matemática, Universidade de Aveiro, Aveiro. Citado na(s) página(s) 18 
Mulaik (2009a) S. A. Mulaik. Foundations of Factor Analysis. Chapman \& Hall/CRC. Citado na(s) página(s) 65

Mulaik (2009b) S. A. Mulaik. Linear Causal Modeling with Structural Equations. Chapman \& Hall/CRC. Citado na(s) página(s) 9

Muthén e Muthén (1998-2010) L. K. Muthén e B. O. Muthén. Mplus User's Guide. Muthén \& Muthén, Los Angeles. Citado na(s) página(s) 11, 33

Paula (2013) G. A. Paula. Modelos de Regressão com Apoio Computacional. Instituto de Matemática e Estatística, Universidade de São Paulo, 2013. Citado na(s) página(s) 12, 28, 33

Paulino et al. (2011) C. D. Paulino, D. D. Pestana, J. A. Branco, J. M. Singer, L. P. Barroso e W. O. Bussab. Glossário Inglês-Português de Estatística. Sociedade Portuguesa de Estatística e Associação Brasileira de Estatística. Citado na(s) página(s) 14, 33

Pilati e Laros (2007) R. Pilati e J. A. Laros. Modelos de Equações Estruturais em Psicologia: Conceitos e Aplicações. Psicologia: Teoria e Pesquisa, 23(2):205-216. Citado na(s) página(s) 3

Pires e Branco (2007) A. M. Pires e J. A. Branco. Introdução aos Métodos Estatísticos Robustos. Sociedade Portuguesa de Estatística, Lisboa. Citado na(s) página(s) 18

R Core Team (2012) R Core Team. R: A Language and Environment for Statistical Computing. R Foundation for Statistical Computing, Vienna, 2012. URL http://www.R-project.org/. ISBN 3-900051-07-0. Citado na(s) página(s) 11, 33

Ribeiro (2010) R. L. F. Ribeiro. Testes de Hipótese Multivariados para Matrizes de Covariâncias em Processos Autocorrelacionados com Aplicações em Controle de Qualidade. Dissertação de Mestrado, Instituto de Ciências Exatas, Universidade Federal de Minas Gerais, Belo Horizonte. Citado na(s) página(s) 10

Rocha (2010) A. V. S. F. Rocha. Estimação Robusta em Modelos Lineares de Equaçôes Simultâneas. Tese de Doutorado, Departamento de Matemática, Universidade de Aveiro, Aveiro. Citado na(s) página(s) $8,20,21,69$

Rosado (2006) F. Rosado. Outliers em Dados Estatísticos. Sociedade Portuguesa de Estatística, Lisboa. Citado na(s) página(s) 19, 28

Rosseel (2012) Yves Rosseel. lavaan: An R Package for Structural Equation Modeling. Journal of Statistical Software, 48(2):1-36. URL http://www.jstatsoft.org/v48/i02/. Citado na(s) página(s) 33

Rousseeuw (1984) P. J. Rousseeuw. Least Median of Squares Regression. Journal of the American Statistical Association, 79(388):871-880. Citado na(s) página(s) 17, 18

Rousseeuw e Leroy (2003) P. J. Rousseeuw e A. M. Leroy. Robust Regression and Outlier Detection. Wiley-Interscience, New York. Citado na(s) página(s) 14, 16, 20

Salibian-Barrera et al. (2006) M. Salibian-Barrera, S. Van Aelst e G. Willems. Principal Components Analysis Based on Multivariate MM Estimators with Fast and Robust Bootstrap. Journal of the American Statistical Association, 101(475):1198-1211. Citado na(s) página(s) 21

Sanches (2005) M. R. Sanches. Indicadores Formativos em Modelos de Equações Estruturais. Dissertação de Mestrado, Instituto de Matemática e Estatística, Universidade de São Paulo, São Paulo. Citado na(s) página(s) 5, 12

Schumacker e Lomax (2004) R. E. Schumacker e R. G. Lomax. A Beginner's Guide to Structural Equation Modeling. Lawrence Erlbaum Associates, Mahwah. Citado na(s) página(s) 31 
Shah e Goldstein (2006) R. Shah e S. M. Goldstein. Use of Structural Equation Modeling in Operations Management Research: Looking Back and Forward. Journal of Operations Management, 24(2):148-169. Citado na(s) página(s) 31

Sharma et al. (2005) S. Sharma, S. Mukherjee, A. Kumar e W. R. Dillon. A Simulation Study to Investigate the Use of Cutoff Values for Assessing Model Fit in Covariance Structure Models. Journal of Business Research, 58(7):935-943. Citado na(s) página(s) 36

Souza (1998) G. S. Souza. Introdução aos Modelos de Regressão Linear e Não-Linear. Embrapa, Brasília. Citado na(s) página(s) 16, 67

Tanaka e Huba (1985) J. S. Tanaka e G. J. Huba. A Fit Index for Covariance Structure Models Under Arbitrary GLS Estimation. British Journal of Mathematical and Statistical Psychology, 38(2):197-201. Citado na(s) página(s) 27

Tanaka e Odaka (1989) Y. Tanaka e Y. Odaka. Influential Observations in Principal Factor Analysis. Psychometrika, 54(3):475-485. Citado na(s) página(s) 28

Tanaka et al. (1991) Y. Tanaka, S. Watadani e S. Ho Moon. Influence in Covariance Structure Analysis: With an Application to Confirmatory Factor Analysis. Communications in Statistics Theory and Methods, 20(12):3805-3821. Citado na(s) página(s) 28

Todorov e Filzmoser (2009) V. Todorov e P. Filzmoser. An Object-Oriented Framework for Robust Multivariate Analysis. Journal of Statistical Software, 32(3):1-47. URL http://www. jstatsoft.org/v32/i03/. Citado na(s) página(s) 33, 67, 70

Venables e Ripley (2002) W. N. Venables e B. D. Ripley. Modern Applied Statistics with S. Springer, New York. URL http://www.stats.ox.ac.uk/pub/MASS4. ISBN 0-387-95457-0. Citado na(s) página(s) 68,69

Widaman e Thompson (2003) K. F. Widaman e J. S. Thompson. On Specifying the Null Model for Incremental Fit Indices in Structural Equation Modeling. Psychological Methods, 8(1):16-37. Citado na(s) página(s) 26

Yohai (1987) V. J. Yohai. High Breakdown-Point and High Efficiency Robust Estimates for Regression. The Annals of Statistics, 15(2):642-656. Citado na(s) página(s) 67

Yuan e Bentler (1998) K. H. Yuan e P. M. Bentler. Structural Equation Modeling with Robust Covariances. Sociological Methodology, 28(1):363-396. Citado na(s) página(s) 20

Yuan e Bentler (2001) K. H. Yuan e P. M. Bentler. Effect of Outliers on Estimators and Tests in Covariance Structure Analysis. British Journal of Mathematical and Statistical Psychology, 54 (1):161-175. Citado na(s) página(s) 13

Yuan e Hayashi (2010) K. H. Yuan e K. Hayashi. Fitting Data to Model: Structural Equation Modeling Diagnosis using Two Scatter Plots. Psychological Methods, 15(4):335. Citado na(s) página(s) 28 